Vie Bgeritive und Schal tungen 3119:

Parallelschilten ron

Wochselstrom - Maschinen

Fon

Wemer Shim

2 write thilage 


\title{
Meßgeräte und Schaltungen zum \\ Parallelschalten von \\ Wechselstrom-Maschinen
}

\author{
Von \\ Werner Skirl \\ Oberingenieur
}

Zweite

umgearbeitete und erweiterte Auflage

Mit 30 Tafeln, 30 ganzseitigen Schaltbildern

und 14 Textbildern

\author{
Berlin \\ Verlag von Julius Springer \\ 1923
}




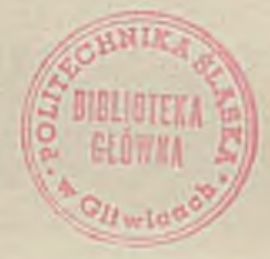

130362

Alle Rechte, insbesondere das der U'bersetzung in fremde Sprachen, vorbehalten. 


\section{Vorwort zur ersten Auflage.}

Die vorliegende Arbeit schließt sich in der Behandlungsweise des Stoffes eng an das von mir herausgegebene Buch „Meßgeräte und Schaltungen für Wechselstrom-Leistungsmessungen" an. Sie ist ebenfalls unmittelbar auf die Bedürfnisse der Praxis zugeschnitten und wird daher dem ausführenden Ingenieur besonders willkommen sein. Aber auch der Studierende wird das Buch mit Vorteil bei der Ausarbeitung von Projekten benutzen können, da er in ihm die Schaltungen so findet, wie sie tatsächlich in der Praxis ausgeführt werden können.

Da über die theoretischen Verhältnisse beim Parallelschalten von synchronen Wechselstrom-Maschinen in der Literatur bereits genügend Material vorhanden ist, schien es nicht angebracht, hier näher auf cliese einzugehen. Es sei in dieser Hinsicht auf das vorzügliche "Lehrbuch der Elektrotechnik" von Prof. Dr. A. Thomälen hingewiesen. Auf die Entwicklungen dieses Lehrbuches aufbauend, beginnt das vorliegende Buch unmittelbar mit der Betrachtung der Vorgänge, wie sie beim Parallelschalten der Maschinen tatsächlich auftreten. Um das Verständnis zu erleichtern, werden hierbei zunächst dio bei Gleichstrom-Maschinen auftretenden Erscheinungen beschrieben, so daß hierdurch ein einfacher Utbergang zu den schwierigeren Verhältnissen bei Wechselstrom geschaffen wird. In zweiten Abschnitt sind die Ausführungsmöglichkeiten der Parallelschaltung angegeben und miteinander kritisch verglichen. Hieran schließt sich ein Abschnitt über die technischen Hilfsmittel zum Parallelschalten an, in dem dic wichtigsten modernen Apparate zum Parallelschalten beschrieben sind. Ältere Apparate sind nur soweit behandelt, als es zum Verständnis der neueren Einrichtungen erforderlich ist. Um die richtige Auswahl der Meßgeräte in jedem Falle zu ermöglichen, ist ein besonderer Abschnitt über die Auswahl der Meßgeräte beigefügt, in dem die Wirkungsweise der Apparate kritisch betrachtet ist. Im vierten Abschnitt sind dann dio vollständigen Schaltungen angegeben. Dic Schaltbilder sind nach den bei den Siemens-Schuckert-Werken geltenden Normen durchgebildet. Neuartig ist die Schaltweise mit dem vom Verfasser angegebenen Umkehrtransformator, der es ermöglicht, die schal- 
tungstechnischen Vorteile der Dunkelschaltung mit den betriebstechnischen Vorteilen der Hellsehaltung zu vereinigen. Um bei den vielen Schaltmöglichkeiten einen klaren Uberblick zu geben, ist auch hier wieder eine Betrachtung über die Auswahl der passenden Schaltung führend. Im fünften Abschnitt ist eine neue, von Dr. Michalke angegebene Einrichtung zum selbsttätigen Parallelschalten beschrieben. Hieran schließt sich noch ein Abschnitt über die Kontrolle fertiger Schaltungen an. Zum Schlusse ist die elektrische Befehlsübertragung zwischen Schaltbühne und Maschinenraum beschrieben. Da dieso Einrichtungen dem Starkstromtechniker weniger bekannt sind, schien eine eingehendere Behandlung dieser Apparate wünschenswert, um so mehr, als hier manche bekannten Schaltungen in einer für den Starkstromtechniker neuen Weise benutzt werden.

Charlottenburg, Mai 1921.

Werner Skirl.

\section{Vorwort zul zweiten Auflage.}

Die zweite Auflage des Buches wurde durch vielfache Erweiterungen ergänzt. Die verschiedenen Schaltmöglichkeiten sind durch Einteilung in direkte, halbindirekte und indirekte Schaltungen schärfer gegliedert worden. Bei dieser Gelegenheit wurde die vom Verfasser angegebene Umkehrschaltung, dic in der ersten Auflage erst nachtrïglich bei der Bearbeitung eingefügt wurde, systematisch in den Stoff hineingearbeitet. Dies schien um so mehr wünschenswert, als sich in der Praxis großes Interesse für diese neue Schaltart gezeigt hat. Neu aufgenommen wurde ein Abschnitt über die bei verschiedenartig geschaiteten Haupttransformatoren zu treffenden Maßnahmen.

Die äußere Ausstattung des Buches ist vollkommen geändert worden. Ebenso wie bei der fast gleichzeitig erscheinenden zweiten Auflage des vom Verfasser herausgegebenen Buches „Meßgeräte und Schaltungen für Wechselstrom-Leistungsmessungen " sind die wichtigsten Kernpunkte des Stoffes in selbständige Bildtafeln mit ausführlichen, erläuternden Unterschriften zusammengefaßt worden. Die bisherigen Autotypien sind durchweg durch Schwarzweißzeichnungen ersetzt, wobei bosonders die vom Verfasser entworfenen Bilder der Meßwerke interessieren dürften.

Charlottenburg, März 1923.

Werner Skirl. 


\section{Inhaltsverzcichnis.}

I. Die elektrischen Vorgünge beim Parallelschalten:

Selte

a. Bedingungen für das Parallelschalten . . . . . . . . . . 1

b. Die Ausglcichströme und ihre Wirkungen. . . . . . . . 2

c. Das Belasten der parallel geschalteten Mascline . . . . 3

II. Die Ausfïhrungsmöglichkeiten der Parallelschaltung:

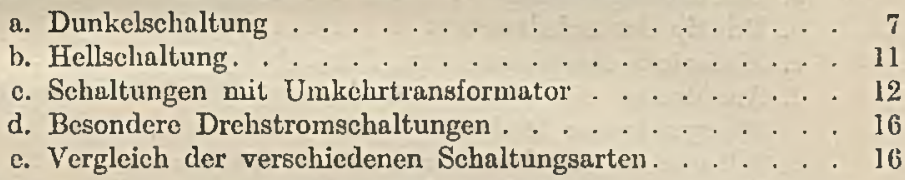

III. Die technischen Hilfsmittel zum Parallelschalten:

a. Elektrische ISinstellvorrichtung für den Regulator der Antriebsmaschine ............. 21

b. Trequenzmesser . . . . . . . . . . . . 21

c. Spannungsmesser . . . . . . . . . . . . 25

d. Phasenlampen . . . . . . . . . . . . . . . . . . . 26

c. Lampenapparato . . . . . . . . . . . . . . . . . . . 29

f. Nullspannungsmesser . . . . . . . . . . . . . . . . . 32

g. Summenspannungsmesser . . . . . . . . . . . . . . . 37

h. Synchronoskope mit schwingendem Zeiger. . . . . . . . 38

i. Synchronoskope mit umlaufendem Zeiger . . . . . . . 43

k. Allgemeines iiber die Auswahl der Meßgeräte . . . . . . 47

l. Vollständige Instrumentsïtze. . . . . . . . . . . . 51

m. Hilfsapparate . . . . . . . . . . . . . . 53

IV. Vollstiondige Schaltungen:

1. Allgemeines über die Auswahl einer passenden Schal. tung . . . . . . . . . . . 56

2. Phasenvergleichung wischen Generator und Sammelschienen . . . . . . . . . . . . 58

a. Dunkelschaltung mit Nullspannungsnesser . . . . . . 58

b. Hellschaltung mit Summenspannungsmesser . . . . . . 61

c. Schaltungen mit Lampenapparat. . . . . . . . . . . . 75

d. Schaltungen mit Synchronoskop . . . . . . . . . . . . 76

3. Phasenvergleichung $\mathrm{z}$ wischen Generator und Genera. tor ................. 84

a. Dunkelschaltung mit Nullspannungsmesser . . . . . 84

b. Gemischte Schaltung mit Nullspannungsmesser und Umkehrtransformator für die Phasenlampe. . . . . . . . 

c. Umkehrschaltung mit Summenspannungsmesser
d. Schaltungen mit Synchronoskop . . . . . . . . . 86

4. Phasenvergleiohung an don Schalterkontakten . 96

a. Dunkelschaltung mit Nullspannungsmesser . . . . . . . 96

b. Gemischte Schaltung mit Nullspannungsmesser und Umkehrtransformator für die Phasenlampe . . . . . . . . 97

c. Unkehrschaltung mit Summenspannungsmesser . . . . . 97

d. Schaltungen mit Synchronoskop . . . . . . . . . . . . 97

e. Direkte Hochspannungsschaltung mit Meßkondensatoren . 103

5. Besondere MaBnahmen bei verschiedenartig geschal-

teten Haupttransformatoren . . . . . . . 106

V. Einrichtungen zum selbsttïtigen Parallelschnlten:

a. Anwendungsgebiete . . . . . . . . . . . 114

b. Prinzip des Schaltmotors . . . . . . . . . . . . . 115

c. Tinfachste Anordnung zum Parallelschalten . . . . . 118

d. Anordnung mit Schleppkontakt zum Parallelschalten bei übersynchroner Drehzahl . . . . . . . . . . . 120

c. Selbsttätige Regelung der Antriebsmaschine. . . . . . 121

f. Verhütung von Fehlschaltungen . . . . . . . . . 124

VI. Schaltungskontrolle:

a. Kontrolle auf richtiges Drehfeld . . . . . . . . . . 126

b. Kontrolle auf richtige Schaltung . . . . . . . . . 127

VII. Elektrische Befehlsübertragung zwischen schaltbühne und Maschinenraum:

๙. Allgemcines . . . . . . . . . . . . . . . 129

b. Glühlampentafeln . . . . . . . . . . . . . . . 129

c. Zeiger-Befehlsapparat mit Sechsspulenmotor, für Gleichstrom 131

d. Zeiger-Befehlsapparat mit Dreispulen-Anker, für Gleichstrom 132

e. Zeiger-Befehlsapparat nach den Wechselstromsystem . . 135

Verzelchnis der Taleln . . . . . . . . . . . . . . . . . 138

Verzeichnis der Schaltbilder vollständiger Parallelschalteinrichtungen . . . . . . . . . . . . . . 139 


\section{Zeichenerklärung für die Schaltbilder.}

\begin{tabular}{|c|c|c|}
\hline & Drehstrom-Generator & $\begin{array}{l}\text { If } I=\text { bereits laufende Maschine } \\
\text { If } I I=\text { zuzuschaltende Maschine }\end{array}$ \\
\hline & $\begin{array}{c}\text { Hauptschalter } \\
\text { mit } \\
\text { Ueberstromauslösung }\end{array}$ & \\
\hline $\int_{1}^{1} \sum_{1}^{1}=$ & $\begin{array}{l}\text { Trennschalter } \\
\text { mit } \\
\text { Hilfskontakt }\end{array}$ & - \\
\hline$\sum_{j}^{u} \sum_{i}^{u}$ & Spannungswandler & $\begin{array}{c}U-V=\text { Primärwickelung } \\
u-v=\text { Sekundärwickelung } \\
J T=\text { Isoliertransformator } \\
U T=\text { Umkehrtransformator }\end{array}$ \\
\hline & Jeßinstrument & $\begin{array}{l}D F=\text { Doppelfrequenzmesser } \\
D V=\text { Doppel-Spannungsmesser } \\
\text { NV }=\text { Nullspannungsmesser } \\
S^{\prime} V=\text { Summenspannungsmesser } \\
S y n=\text { Synchronoskop }\end{array}$ \\
\hline$\infty$ & Glühlampe & $P=$ Phasenlampe \\
\hline - & $\begin{array}{l}\text { Vor- bzw. } \\
\text { Ersatzwiderstand }\end{array}$ & - \\
\hline $\begin{array}{l}(0) \\
(0)\end{array}$ & Steckvorrichtung & nit bzw. ohno Stecker \\
\hline U/R & Schutzerdung & $\begin{array}{l}\text { strichpunktierte Linien } \\
\text { sind Erdungsleitungen }\end{array}$ \\
\hline
\end{tabular}





\section{Die elektrischen Vorgänge beim Parallelschalten.}

\section{a. Bedingungen fiir das Parallelschalten.}

Um das Verständnis der nicht ganz einfachen Verhältnisse beim Parallelschalten von synchronen Wechselstrom-Maschinen zu erleichtern, sind bei den einleitenden Abschnitten zunächst die bei Gleichstrom-Maschinen auftretenden einfacheren Vorgänge besprochen, so daß hierdurch ein leichter Utbergang zu den schwierigeren Verhältnissen bei Wechselstrom gegeben wird.

Soll eine Gleichstrom-Nebenschlußmaschine zu einer anderen bereits im Betriebe befindlichen Maschine parallel geschaltet werden, so bringt man sie zunächst auf ihre normale Drehzahl und erregt sie dann. Ist ihre Spannung vollkommen gleich der Spannung der bereits auf das Netz arbeitenden Maschine, so legt man dic Schalter ein. Da die beiden parallelgeschalteten Maschinen mit den gleichen Polen aneinander geschaltet, also elektrisch gegeneinander geschaltet sind, heben sich ihre Spannungen auf. Die hinzugeschaltete Maschine läuft daher zunächst leer am Netz.

Soll eine Wechselstrom-Maschine parallel geschaltet werden, so muß zunächst die Frequenz, dann die Größe und endlich die Phase der Spannungen übereinstimmen. Die Frequenz ist unmittelbar von der Drehzahl abhängig. Die neu hinzuzuschaltende Maschine muß daher eine ganz genau bestimmte, der jeweiligen Frequenz entsprechende Drehzahl haben. Zu dieser Bedingung, die an sich mechanisch schwer durchführbar ist, da es sich um eine absolut genaue Übereinstimmung der Drehzahlen handelt, kommen noch die weiteren Bedingungen, daß die Effektivspannungen genau die gleiche Größe haben und daß außerdem die Spannungskurven in Phase sind. Sind diese Bedingungen erfüllt, so kann man die Schalter schließen und damit die Maschine mit dem Netz verbinden. Die neu hinzugeschaltete Maschine läuft dann ebenso wie die Gleichstrom-Maschine leer am Netz, da sich die Momentanwerte der Spannungen in jedem Augenblick gegenseitig aufheben.

$3 \mathrm{kjr}$, Parallelschalten, 2, Aufl. 


\section{b. Die Ausgleichströme und ihre Wirkungen.}

Wenn man eine Gleichstrom-Maschine parallel zu einer underen geschaltet hat, läuft sie zunächst leer am Netz, solange ihre Drehzahl und ihre Erregung unverändert bleibt Wächst durch einen Zufall die Drehzahl der Antriebsmaschine, so steigert sich nit dieser die Elektromotorische Kruft der von ihr angetriebenen Gleichstrom-Maschine und es fließt ein Strom, der die voreilende Maschine als Generator belastet und die zurückbleibende als Motor antreibt. Die Folge hiervon ist, daß die voreilende Maschine infolge ihrer Belastung etwas zurückbleibt und die zurückbleibende Maschine infolge ihrer Entlastung etwas voreilt, bis die Verschiedenheit ausgeglichen ist. Bleibt die zugeschaltete Maschine andererseits etwas zurück, so empfängt sie von der bereits laufenden Maschine einen Strom, der sie motorisch beschleunigt. Man nennt diesen von Maschine zu Maschine gehenden Strom den Ausgleichstrom, da er die Verschiedenheiten der beiden parallelgeschalteten Maschinen ausglcicht.

Eine Wechselstrom-Maschine läuft nach dem Parallelschalten zunächst ebenfalls leer am Netz. Es fragt sich nun aber, wie es möglich ist, daß die im Augenblick des Parallelschaltens vorhandene Übereinstimmung der Maschinen in Drehzahl und Phase dauernd aufrecht erhalten bleibt. Die Verhältnisse bei Gleichstrom lassen die richtige Vermutung aufkommen, daß auch hier wieder Ausgleichströme fließen, die die Maschinen im 'Tritt halten. Tatsächlich verursacht auch bei einer Wechselstrommaschine eine mechanische Voreilung des Ankers einen Ausgleichstrom, der die Maschine als Generator belastet, während beim Zurückbleiben des Ankers ein Ausgleichstrom auftritt, der die Maschine als Motor antreibt. Diese Ausgleichströme können aber bei einer Wechselstrom-Maschine naturgemä $ß$ nicht durch eine Änderung der Drehzahl verursacht werden, da diese zur Folge haben müßte, daß die Maschinen vollkommen aus dem Tritt fallen. Die mechanische Voreilung bzw. das Zurückbleiben des Ankers kann sich bei einer Wechselstrom-Maschine vielmehr nur innerhalb einer Polteilung abspielen. Nehmen wir an, daß eine Maschine das Bestreben hat, der anderen etwas vorauszueilen, so werden ihre Ankerdrähte innerhalb der Polteilung relativ zu den Magnetpolen schon etwas weiter nach vorn verschoben sein, als dies bei den übrigen Maschinen der Fall ist. Dies ist aber gleichbedeutend 
mit einer Phasenvoreilung der Spannung der zugeschalteten Maschine. In ähnlicher Weise wird ein Zurückbleiben des Ankers eine Phasemnacheilung der erzeugten Spannung bedeuten. Je nach dem Sinn dieser Phasenverschiedenheit wird der Ausgleichstrom in dem einen oder dem anderen Sinne fließen. Im Augenblick des Parallelschaltens werden diese durch Phasenverschiedenheiten bedingten Ausgleichströme momentan entstehen und den Anker der zugeschalteten Maschine mit einem Ruck in die richtige Stellung vor den Polen drehen. Nachdem dieser Zustand erreicht ist, hören diese Ausgleichströme sofort auf. Sie sind demnach im wesentlichen momentane Stromstöße, aber als solche besonders gefährlich, do sie die Maschinen und ihre Wickelungen mechanisch stark beanspruchen. Um die durch Phasenverschiedenheiten verursachten Ausgleichströme in zulässigen Grenzen zu halten, ist es erforderlich, die Phasenverschiedenheiten vor dem Parallelschalten genau zu messen. Geschieht dies nicht, so läuft man Gefahr, daß die Ausgleichströme eine derartige Größe annehmen, daß sie den Betrieb stören und die Maschinen beschädigen.

Außer durch Phasenverschiedenheiten können auch durch verschiedene Größe der Spannungen der zuzuschaltenden und der bereits laufenden Maschine Ausgleichströme entstehen. Die durch Spannungsdifferenzen verursachten Ausgleichströme sind aber ihrer Natur nach wattlos. Sie wirken, wie aus dem folgenden Abschnitt hervorgeht, nicht unmittelbar auf die Maschinen zurück, sondern stellen lediglich eine wenn auch unerwünschte Strombelastung der Maschinenwickelungen und Schalterkontakte dar. Diese wattlosen Ausgleichströme fließen dauernd, solange die Erregung nicht geändert wird. Sie sind ungefährlich, wenn die Spannungsdifferenzen nicht allzu groß sind und können stets durch entsprechende Einstellung der Erregung der Maschinen in kleinen Grenzen gehalten bzw. zum Verschwinden gebracht werden, ohne daß hierdurch das Zusammenarbeiten der Maschinen beeinflußt wird.

\section{c. Das Belasten dor parallelgeschalteten Maschine,}

Um die Gleichstrom-Maschine, dic nach erfolgter Parallelschaltung zunächst leer am Netz läuft, zu belasten, verstärkt man ihre Erregung und damit ihre Elektromotorische Kraft, so daß diese die Spannung der bereits im Betrieb befindlichen 
Maschine überwiegt. Infolgedessen liefert die neu hinzugekommene Maschine einen Strom, d. h. sie wird belastet. Die bisher der Gleichstrom-Maschine zugeführte mechanische Leistung reicht dann nicht mehr aus. Die antreibende Dampfmaschine wird da. her verzögert werden und das Gewicht ihres Regulators wird heruntersinken. Hierdurch wird die Dampfzufuhr und damit die mechanische Leistung der Dampfmaschine vergrößert, bis die zugeführte mechanische Leistung wieder gleich der verbrauchten elektrischen Leistung ist. Bei der parallelgeschalteten Nebenschlußmaschine wird also die Belastung durch die Erregung verändert. Die Gleichstrom-Maschine wirkt hierbei auf die Dampfmaschine derart zurück, daß sich die Drehzahl und die zugeführte mechanische Leistung nach der geforderten elektrischen Leistung ändert, d. h. die Dampfmaschine gibt das her, was die Gleichstrom-Maschine fordert.

Bei Wechselstrom liegen die Verhältnisse ganz anders. Würde man hier nach erfolgter Parallelschaltung die Erregung der neu hinzugeschalteten Maschine vergrößern, so würde die Maschine wohl einen Strom liefern, aber ein Blick auf den Leistungsmesser zeigt uns, daß die Maschine keine Leistung übernimmt. Der gelieferte Strom ist demnach wattlos. Man kommt, wenn man dic Verhältnisse vom rein mechanischen Standpunkt aus übersieht, zum selben Schlusse. Die von der Dampfmaschine gelieferto Leistung hängt lediglich von der Dampfzufuhr, d. h. von der jeweiligen Stellung des Regulatorgewichts, also von der Drehzahl der Dampfmaschine ab. Da aber die Drehzahl durch die elektrischen Bedingungen vollkommen festgelegt ist und sich daher nicht änderı kann, bleibt das Regulatorgewicht dauernd in derselben Stellung. Wie man auch die Erregung andert, die Dampfmaschine liefert immer nur die teerlaufsarbeit. Hieraus folgt, daß bei einer parallelgeschalteten Wechselstrommaschine die Belastung nicht von der elektrischen Seite her erfolgen kann, sie muß vielmehr von der antreibenden Dampfmaschine aus eingestellt werden. Die Einstellung der Leistung der Dampfmaschine erfolgt durch eine Verstellung des Regulatorgewichts. Durch eine Vergrößerung des Regulatorgewichts erreicht man, daß die Dampfmaschine bei derselben durch die Periodenzahl des Netzes ihr aufgezwungenen Drehzahl eine größere Menge Dampf erhält. Die Vermehrung des Dampfzutrittes hat eine 
mechanische Voreilung des Ankers der Wechselstrom-Maschino zur Folge, die ihrerseits einer elektrischen Belastung des Ankers entspricht. Die elektrische Leistung einer parallelgeschalteten Wechselstrom-Maschine kann also nur durch Änderung der zugeführten mechanischen Leistung verändert werden, $d . h$. dic Wechselstrom-Maschine kann nur das hergeben, was ihr von der Dampfmaschine bei der jeweiligen Regulatorstellung zugeführt wird.

Für das Parallelschalten yon Wechselstrom-Maschinen haben diese Verhältnisse insofern Bedeutung, als es von ihnen abhängt, ob die hinzugeschaltete Maschine unmittelbar nach dem Parallelschalten als Generator Last übernimmt, oder als Motor vom Netz angetrieben wird. Ist nämlich die Frequenz der parallel zu schaltenden Maschine etwas höher als die des Netzes, so wird die Maschine sofort nach dem Parallelschalten bestrebt sein, die frühere, etwas höhere Drehzahl beizubehalten. Sie kann dies nicht, da sie durch die Ausgleichströme im Tritt gehalten wird. Immerhin aber wird ihr Anker infolge der überschüssigen Antriebskraft innerhalb der Polteilung nach vorn verschoben. Diese Voreilung entspricht aber nach dem Vorausgegangenen einer elektrischen Belastung des Ankers als Generator, d. h. mit anderen Worten, eine parallelgeschaltete Wechselstrom-Maschine nimmt sofort nach dem Einschalten Last auf, wenn sie bei einer etwas zu hohen Frequenz parallelgeschaltet wird. Diese Belastung bleibt dauemd bestehen, solange die Regulatorstellung der Antriebsmaschine nicht geändert wird. In analoger Weise wird dann, wenn eine Maschine bei zu kleiner Frequenz eingeschaltet wird, ihr Anker hinter der normalen Stellung zurückbleiben. Dies bedeutet aber, daß die Maschine als Motor läuft und vom Netz aus angetrieben wird. Auch dieser Zustand ist dauernd, bis der Regulator der Antriebsmaschine entsprechend verstellt wird. Da meistens die bereits im Betriebe befindlichen Maschinen schon stark belastet sind, ehe man eine neue Maschine in Betrieb nimmt, wird man eine weitere Belastung des Netzes durch die zugeschaltete Maschine gern vermeiden. Man schaltet vielmehr, wenn es die Betriebsverhältnisse ermöglichen, stets bei etwas übersynchronem Gang, also bei etwas zu hoher Frequenz, ein. 


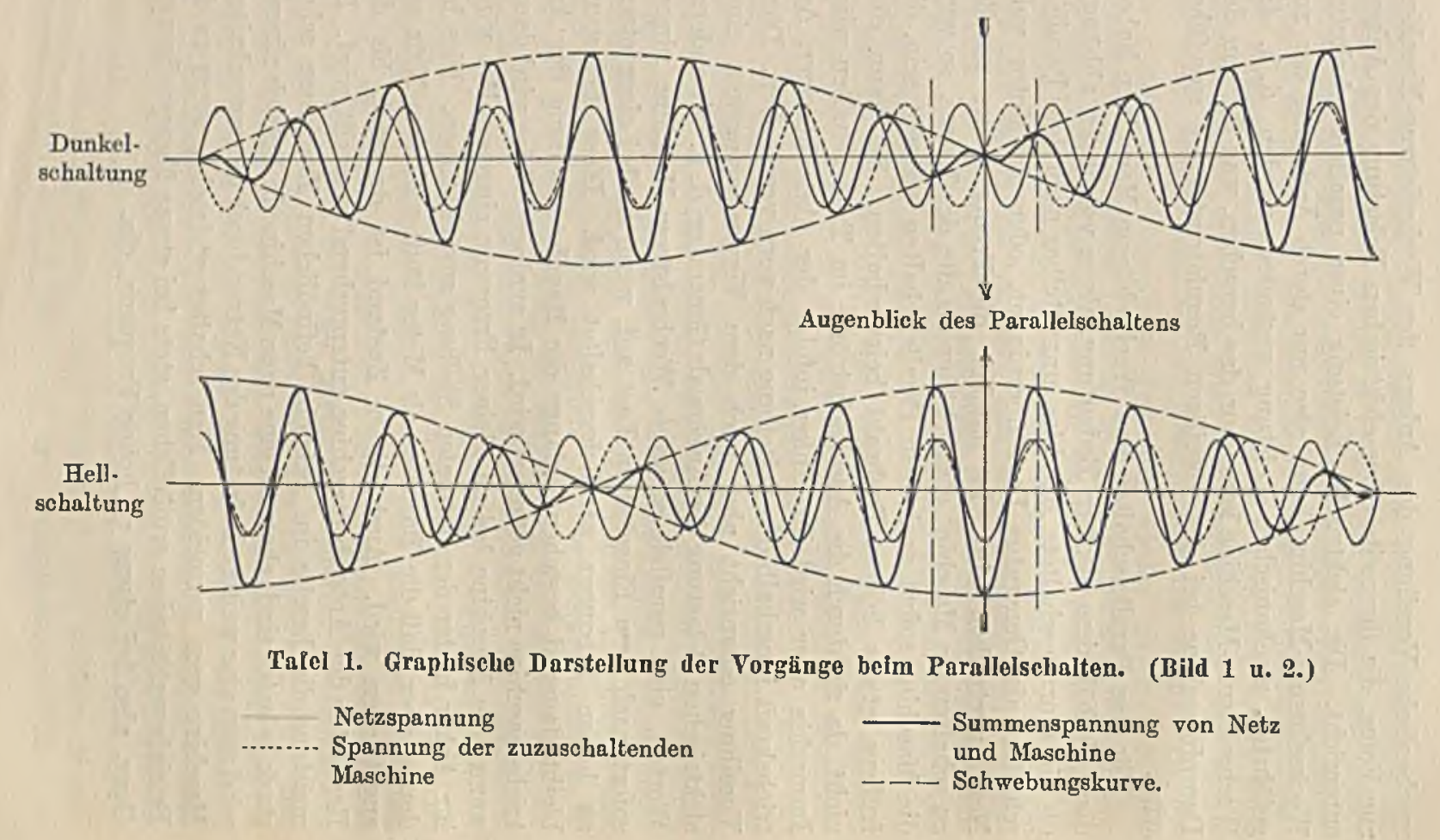




\section{Die Ausführungsmöglichkeiten der Parallelschaltung.}

\section{a. Dunkelschaltung.}

Wir hatten im vorhergehenden Abschnitt gesehen, daß bei einer parallel zu schaltenden Wechselstrom-Maschine außer der Spannung noch die Frequenz und die Phase genau mit den entsprechenden Größen der bereits im Betriebe befindlichen Maschine übereinstimmen müssen. Um eine Maschine neu in Betrieb zu nehmen, wird man sie zunächst annähernd auf die richtige Drehzahl bzw. Frequenz bringen, dann erregt man die Maschine so, daß ihre effektive Spannung gleich der Netzspannung ist. Die Einstellung auf gleiche Phasen scheint auf den ersten Blick wesentlich schwieriger, aber man kommt auch hier durch eine cinfache Utberlegung rasch zum Ziel. Da man ganz unabhängig von den sonstigen Schaltungsverhältnissen zwei beliebige Punkte miteinander verbinden kann, wenn sie genau das gleiche Potential haben, kann man den Hauptschalter (vgl. Bild 3), der die neue Maschine mit dem Netz verbindet, ohne weiteres einlegen, wenn zwischen den zu verbindenden Kontakten keime Spannungen vorhanden sind. Das cinfachste Mittel, um das Vorhandensein einer Spannung zu crkennen, ist eine Glühlampe. Man schaltet also an die Kontakte der Schalter je cine für die Netzspannung bemessene Glühlampe. Leuchten die Lampen auf, so bestcht zwischen den Kontakten des Schalters eine Spannung und man darf demgemäß nicht einschalten. Verlöschen die Glühlampen, so ist zwischen den Schalterkontakten keine oder nur eine sehr kleine Spannung vorhanden. Man könnte daher in diesem Falle den Schalter einlegen. Wenn man diese Schaltung tatsächlich ausführt, so zeigt sich, daß die Lampen periodisch aufleuchten und verlöschen. Die Zeiträume, in denen dies erfolgt, werden um so größer, je mehr die Frequenz der in Betrieb zu nehmenden Maschine mit der des Netzes übereinstimmt. Ein dauerndes Verlöschen der Lampen läßt sich praktisch nicht erreichen, da dies voraussetzen würde, daB die Frequenzen vom Zeitpunkt des Verlöschens der Lampen an mathematisch genau gleich bleiben. Man muß sich daher begnügen, wenn die Lampen in größeren Zciträumen aufleuchten und verlöschen. Man legt den Schalter dann in einem 


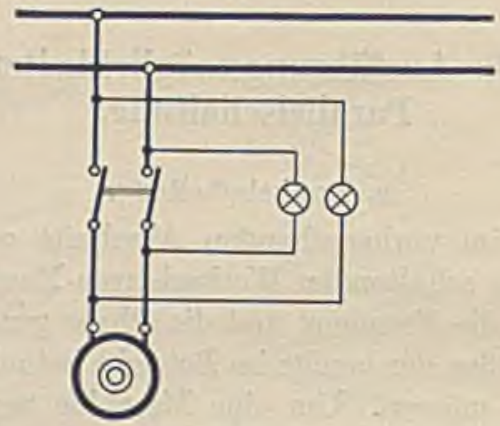

Bild 3. Direkte Schaltung.

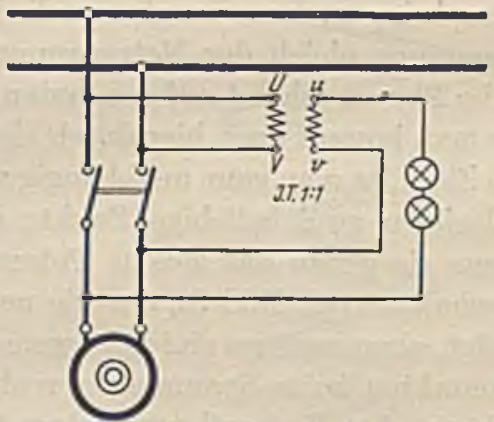

Bild 4. Halbindirekte Schaltung.

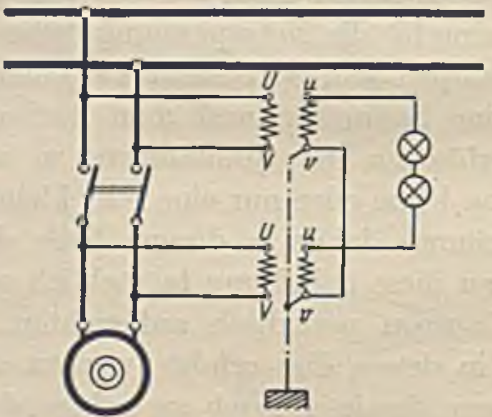

Bild 5. Indirekte Schaltung.

Die an den Phasenlampen auftretende Höchstspannung ist bei der direkten und halbindirekten Schaltung gleich der doppelten Netzspannung, bei der indirekten Schaltung dagegen $2 \times 110$ Volt.

Tafel உ. Ausfïhrungsmöglichkeiten der Dunkelschaltung. 
Zeitpunkt ein, in dem die Lampen dunkel sind. Die bei dieser Phasenabgleichung auftretenden elektrischen Vorgängo gehen aus dem oberen Kurvenbild auf Tafel 1 hervor. Die ausgezogene Sinuskurve ist die Spannungskurve des Netzes, die gestrichelte Kurve die Spannungskurve des hinzuzuschaltenden Generators. Die beiden Kurven unterscheiden sich entsprechend den nicht genau übereinstimmenden Drehzahlen der Generatoren nur durch eine geringe Frequenzabweichung. Infolge dieser Trequenzabweichung ändert sich dauernd die Phasenverschiebung zwischen der Generatorspannung und der Netzspannung und demgemäß auch die Summe dieser beiden Spannungen. Die resultierende Summenkurve ist stark ausgezeichnet. Diese Kurve zeigt Interferenzerscheinungen ähnlich den Schwebungen, dic bei annähernd gleichen Wellenlängen in der Optik und Akustik auftreten. Der Augenblick des Parallelschaltens ist dann gekommen, wenn die Netzspannung und die Spannung der hinzuzuschaltenden Maschine einander gerade entgegengesetzt und gleich groß sind, so daß sie einander aufheben. Dies ist der Augenblick, in dem die Lampen verlöschen und in dem der Schalter eingelegt werden muß. Ist der Schalter eingelegt, so setzen sofort die Ausgleichströme ein, die die Maschinen auf absolut gleiche Periodenzahl bringen und dauernd auf dieser erhalten.

Bei der praktischen Ausführung der Dunkelschaltung gibt cs drei Möglichkeiten, die direkte, die halbindirekte und die indirekte Schaltung. Bei der direkten Schaltung liegen die Phasenlampen unmittelbar an den zu vergleichenden Spannungen, wie es Bild 3 auf Tafel 2 zeigt. Bei der halbindirekten Schaltung wird auf der Sammelschienenseite ein im Verhältnis 1 : 1 übersetzender Isoliertransformator benutzt. Durch diesen werden die Sammelschienen elektrisch vollkommen von der Parallelschalteinrichtung getrennt. Man kann daher ohne weiteres den einen Pol der Sekundärwicklung des Isoliertransformators unmittelbar mit einer Maschinenleitung verbinden und die beiden in Reihe geschalteten Phasenlampen in die andere Leitung verlegen. Durch die einpolige Verbindung ergeben sich für die Ausführung der Schaltung mit Hilfssammelschienen wesentliche Vereinfachungen (vgl. Schaltbild 2 auf S. 65). Außerdem fallen die Schwierigkeiten weg, die bei der direkten Schaltung durch die zu den Phasenlampen parallel geschalteten Meßinstrumente ent- 


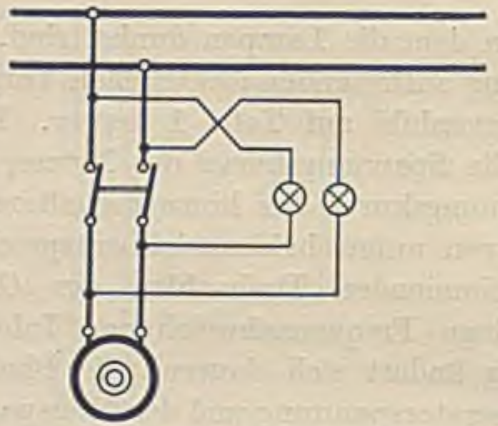

Bild 6. Direkto Schaltung.

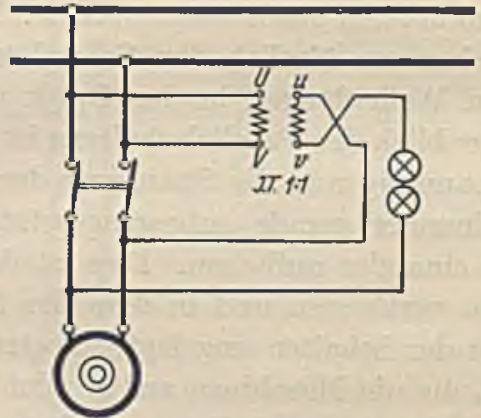

Bild 7. Halbindirekte Schaltung.

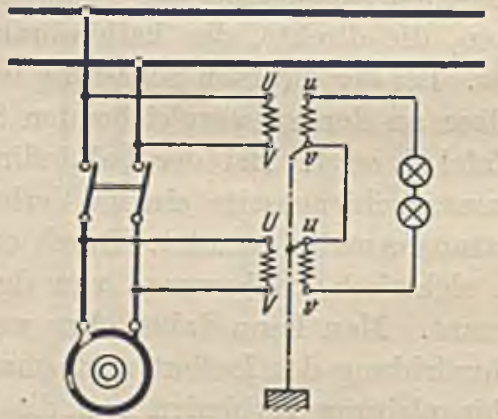

Bild 8. Indirektc Schaltung.

Die an den Phasenlampen auftretende Höchstspannung ist bei der direkten und halbindirekten Schaltung gleich der doppolten Netzspannung, bei der indirekten Schaltung dagegen $2 \times 110$ Volt. 
stehen (vgl. S. 59). Bild 5 zeigt die indirekte Schaltung mit Spannungswandlern, wic sie vorzugsweise bei Hochspannung ausgeführt wird. Dic beiden Spannungswandler übersetzen hicrbei in jedem Falle auf eine Sekundärspannung von 110 Volt, so daß die Meßeinrichtung nur Niederspannung führt. Die an den Phasenlampen auftretende Höchstspannung beträgt $2 \times 110$ Volt. Nach den Sicherheitsvorschriften des Verbandes Deutscher Elektrotechniker müssen hierbei die Sekundärwickelungen der Spannungswandler stets geerdet werden. Charakteristisch für die Dunkelschaltung ist es, daß diese Erdung immer an gleichnamigen Polen der Meßwandler erfolgt.

\section{b. Ifellschaltung.}

Man kann die zur Phasenabgleichung benutzten Glühlampen auch mit überkreuzten Leitungen anschließen, wic es Bild 6 auf Tafel 3 zeigt. In diesem Falle wird die Spannung des hinzuzuschaltenden Generators über die Glühlampen hinweg in Reihe mit der Netzspannung geschaltet. Die Schaltung der Hauptleitung wird natürlich hierdurch nicht geändert, so daß in bezug auf diese nach wie vor Generator und Netz gegeneinander geschaltet sind. Wenn jetzt die Spannung zwischen den Schalterkontakten gleich Null ist, weil sich die gegeneinander geschalteten Spannungen aufheben, so wird in Kreise der Phasenlampen die doppelte Spannung auftreten, da sich in diesem Kreise die Spannung des Generators zu der Netzspannung addiert; mit anderen Worten, bei dieser Schaltung werden die beiden in Reihe geschalteten Glühlampen im Moment der Phasengleichheit mit der vollen Spannung brennen. Da der Schalter hierbei in dem Augenblick eingelegt wird, in dem die Lampen mit voller Lichtstärke brennen, nennt man diese Schaltung die Hellschaltung. Die hierbei auftretenden elektrischen Verhältnisse ergeben sich aus dem unteren Kurvenbild auf Tafel 1. Das Kurvenbild ist ohne weiteres verständlich, wenn man beachtet, daß die im Kreise der Phasenlampen wirkende Spannung der zuzuschaltenden Maschine durch die Utberkreuzung der Leitungen um $180^{\circ}$ herumgeklappt wird.

Für die praktische Ausführung der Hellschaltung gibt es ebenso wie bei der Dunkelschaltung drei Möglichkeiten, die direkte, die halbindirekte und die indirekte Schaltung. Bei der in Bild 6 
dargestellten direkten Schaltung liegen die Phasenlampen unmittelbar an den zu vergleichenden Spannungen. $\mathrm{Zu}$ beachten ist hierbei der durch die Überkreuzung angedeutete wechselpolige Anschluß. Bei der in Bild 7 gezeigten halbindirekten Schaltung jst auf der Sammelschienenseite des Schalters wieder ein im Verhältnis 1:1 übersetzender Isoliertransformator eingeschaltet. Durch diesen werden die Sammelschienen elektrisch vollkommen von der Parallelschalteinrichtung getrennt. Man kann daher wieder den einen Pol der Sekundärwickelung des Isoliertransformators unmittelbar mit einer Maschinenleitung verbinden und die beiden in Reihe geschalteten Phasenlampen in die andere Leitung legen. Hierdurch ergeben sich für die Ausführung der Schaltung wesentliche Vereinfachungen (vgl. Schaltbild 7 auf S. 70). Ebenso fallen wieder die Schwierigkeiten weg, die bei der direkten Schaltung durch dic zu den Phasenlampen parallel geschalteten Meßinstrumente entstehen (vgl. S. 61). Bild 8 zeigt die indirekte Schaltung mit Spannungswandlern. Die Sekundärspannung dieser Spannungswandler beträgt stets 110 Volt, so daß an den Phasenlampen eine Höchstspannung von $2 \times 110$, also 220 Volt, auftritt. Da die Sekundärwickelungen der Spannungswandler in Reihenschaltung liegen, kann hierbei die Erdung nur an dem gemeinsamen Punkt der beiden Wickelungen erfolgen. Die Erdung ist also im Gegensatz zu der Dunkelschaltung wechselpolig. Hierdurch ergeben sich für die Ausführung der Schaltungen erhebliche Schwierigkeiten, sobald mehr als zwei Maschinen wahlweise untereinander parallel geschaltet werden sollen, da eben die Vertauschung der Pole nur zwischen zwei Maschinen möglich ist.

\section{c. Schaltungen mit Umkehrtransiormator.}

Die schaltungstechnischen Schwierigkeiten, dic bei der Hellschaltung beim unmittelbaren Vergleich von mehr als zwei Maschinen durch die wechselpolige Erdung entstehen, führten den Verfasser zu einer neuen Schaltung, die im nachstchenden als Umkehrschaltung bezeichnet ist. Die neue Schaltung ist in Bild 9 dargestellt. Hierbei sind die Spannungswandler genau so wie bei der Dunkelschaltung geschaltet (vgl. Bild 5). Die Sekundärwickelungen sind demgemäß elektrisch gegeneinander geschaltet und gleichpolig geerdet. Um trotz dieser Dunkelschaltung der 


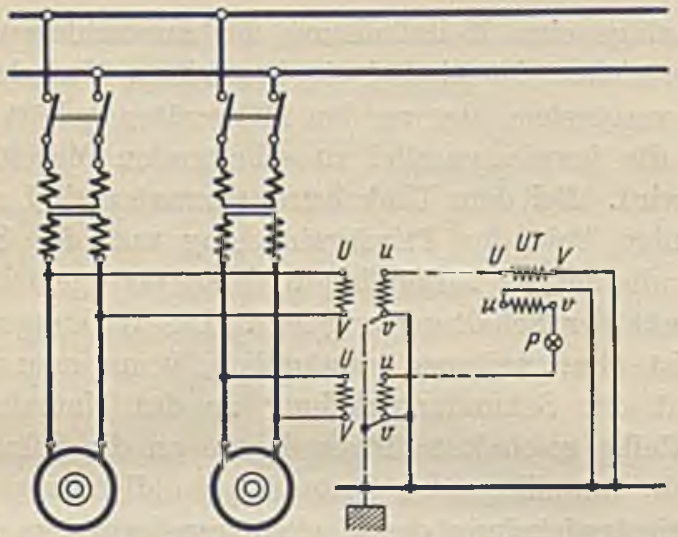

Bild 9. Umkehrschaltung. Die Maschinen-Spannungswandler sind hierbei genau wie bei der Dunkelschaltung gegeneinander geschaltet und gleichpolig geerdet. Durch oinen besonderen Umkehrtransformator $U T$, der mit den Meßgeräton zusammen auf die jeweils parallel zu schaltenden Maschinen umgeschaltet wird, wird die Schaltung unmittelbar vor den Meßgeräten in cine Hellschaltung umgekehrt.

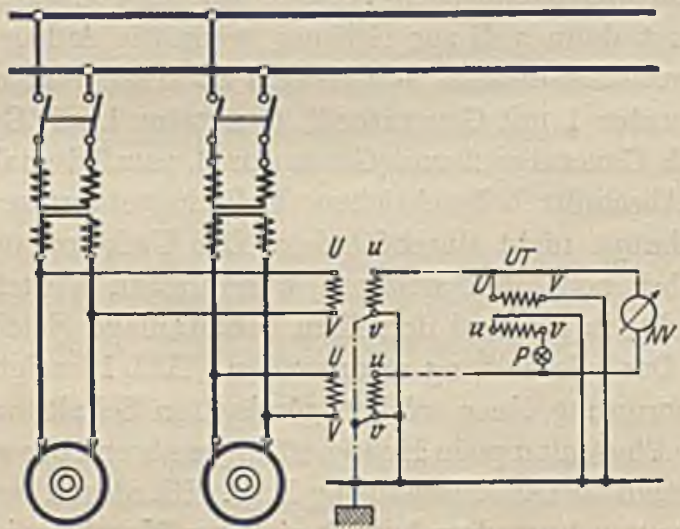

Bild 10. Gemischte Schaltung. Das MeBinstrument $(N V)$ liegt hierbei in der normalen Dunkelschaltung, während dic Phasenlampe unter Zwischenschaltung eines Umkebrtransformators in Hellschaltung arbeitet.

Tafel 4. Schaltungen mit Umkehrtransformator. 
Maschinenanlage eine Hellschaltung der angeschlossenen Meßgeräte zu erreichen, ist hierbei ein besondercr Umkehrtransformator UT vorgesehen, der zu den Meßgeräten gehört und mit diesen auf die jeweils parallel zu schaltenden Maschinen umgeschaltet wird. Bei dem Umkehrtransformator sind stets zwei ungleichnamige Pole der Primärwickelung und der Sekundärwickelung miteinander verbunden und an den gemeinsam geerdeten Punkt der Schaltung angelegt. Die Wirkungsweise der Schaltung ist ohne weiteres verständlich, wenn man beachtet, daß die mit der Sekundärwickelung $u v$ des Umkehrtransformators in Reihe geschaltete Phasenlampe an die Sekundärseite des im Bilde untenliegenden Spannungswandlers angeschlossen ist. Die Primärwickelung des Umkehrtransformators liegt mit vertauschten Polen an dem Spannungswandler der anderen Maschine. Die Spannung des oberen Spannungswandlers wird infolgedessen in den Kreis der Phasenlampe hineintransformiert, so daß in der Phasenlampe dieselben Schwebungen entstehen wie bei der normalen Hellschaltung. Da der Umkehrtransformator im Verhältnis 1:1 übersetzt, ist die an der Phasenlampe auftretende Höchstspannung $2 \times 110$ Volt.

Die schaltungstechnischen Vorteile des Umkehrtransformator's kommen erst dann voll zur Geltung, wenn die Anlage drei und mehr Generatoren enthält, wie es Bild 83 zeigt, und man wahlweise Generator 1 mit Generator 2, Generator 1 mit Generator 3 und endlich Generator 2 und Generator 3 parallelschalten muß. Die unter Abschnitt $b$ beschriebene Hellschaltung wäre in diesem Falle überhaupt nicht durchführbar. Der Umkehrtransformator gewährt aber noch den weiteren wesentlichen Vorteil, daß er es ermöglicht, in ein und derselben Schaltanlage gleichzeitig die Hell- und Dunkelschaltung anzuwenden. Bild 10 zeigt die prinzipielle Anordnung einer solchen gemischten Schaltung. Hierbei arbeitet die Phasenlampe in Hellschaltung, während das anzeigende Meßinstrument in Dunkelschaltung liegt. Hierdurch werden eine ganze Reihe von besonders betriebssicheren Kombinationen möglich, die bisher nicht ausführbar waren (vgl. die Instrumentsätze auf S. 50). Dieser Vorteil führt dazu, die Schaltung mit Umkehrtransformator neuerdings auch bei der Phasenvergleichung zwischen Generator und Sammelschienen anzuwenden. 


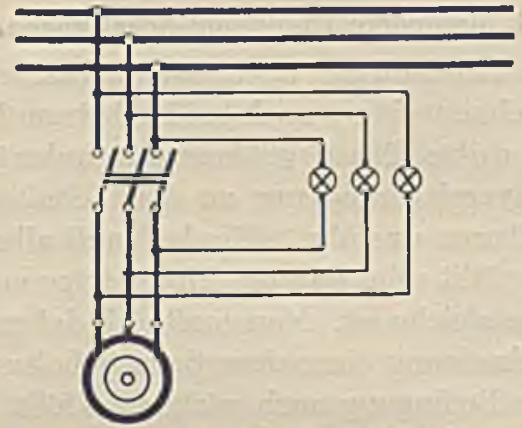

Bild 11. Dreiphasige Dunkelschaltung.

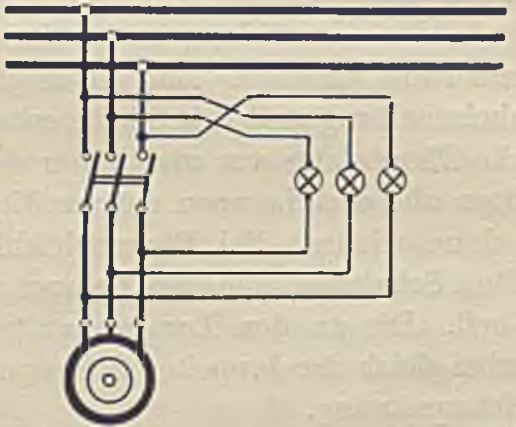

Bild 12. Dreiphasige Hellschaltung.

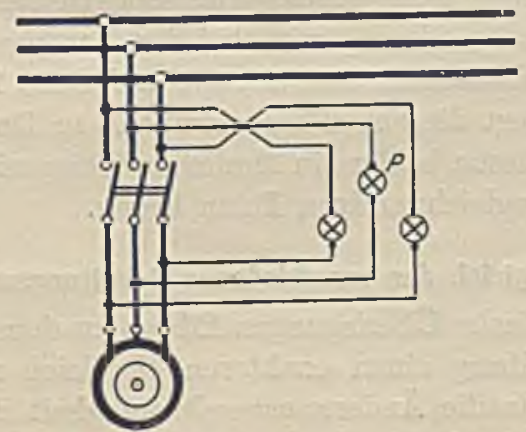

Bild. 13. Umlaufschaltung.

Die an den Lampen auftretende Höchstspannung ist gleich der doppelten Sternspannung, also das 1,15 fache der Netzspannung.

Tafel 5. Besondere Drehstrom-Schaltungen. 


\section{d. Besondere Drehstrom-Schnitungen.}

Die in den Abschnitten $a, b$ und $c$ beschriebenen Schaltungen können ohne weiteres auch bei Drehstrom benutzt werden, wenn man die dritte Phase vollkommen unberücksichtigt läßt und die Phasenvergleichung nur an den Schalterkontakten der ersten beiden Phasen ausführt. Hierbei muß allerdings stets die Voraussetzung erfüllt sein, daß dic Phasenfolge auf beiden Seiten des Schalters die gleiche ist. Man muß sich daher in jedem Falle bei der Inbetriebsetzung einer derartigen Schaltung daron überzeugen, ob diese Bedingung auch wirklich erfüllt ist (vgl. S. 126). Bei den hierzu erforderlichen Kontrollmessungen ist es oft zweckmäßig, eine provisorische dreiphasige Hilfsschaltung auszuführen, die unabhängig von der eigentlichen, in der Anlage eingebauten Parallelschaltvorrichtung arbeitet. Bild 11 zeigt eine derartige dreiphasige Dunkelschaltung und Bild 12 die entsprechende Hellschaltung. Ist die Phasenfolge vor und hinter dem Schalter die gleiche, so leuchten alle drei Lampen gleichzeitig periodisch auf und verlöschen dann wieder. Bei Phasengleichheit bleiben, je nach der gewählten Schaltung, alle drei Lampen dunkel oder sie brennen gleich hell. Die an den Lampen auftretende Höchstspannung ist hierbei gleich der doppelten Sternspannung, also das 1,15fache der Netzspannung.

In Bild 13 ist noch eine Umlaufschaltung angegeben, bei der die Glühlampen nicht gleichzeitig, sonderm nacheinander aufleuchten. Diese Anordnung wird bei den Lampenapparaten mit umlaufendem Lichtschein benutzt, die auf Seite 29 beschrieben sind. Hierbei liegt die eine Phasenlampe $P$ in Dunkelschaltung, während die anderon beiden, in ähnlicher Weise wie bei der Hellschaltung, an ungleichnamigen Polen liegen.

\section{o. Vergleich der verschiedenen Schaltungsarten.}

Rein theoretische U'berlegungen führen zu dem Schlusse, daß die Dunkelschaltung einen exakteren Vergleich zuläßt als die Hellschaltung, da die Änderungsgeschwindigkeit der resultierenden Spannung, die durch die gestrichelte Schwebungskurve auf S. 6 dargestellt wird, bei der Dunkelschaltung im Augenblick des Parallelschaltens wesentlich größer ist. Eine geringfügige $\mathrm{Ab}$ weichung von der Phase wird daher bei der Dunkelschaltung eine größere Änderung der resultierenden Spannung zur Folge 
haben als bei der Hellschaltung. In der Praxis werden jedoch die Verhältnisse durch die beim Parallelschalten auftretenden Nebenumstände wesentlich geändert. Die den theoretischen Erwägungen zugrunde gelegten Schwebungskurven gelten nur für den Fall, daß die beiden zu vergleichenden Spannungen vollkommen gleich groß sind. Tatsächlich wird diese Bedingung aber meistens nicht genau crfüllt sein, da sich die Spannungen mit der zu regelıden Frequenz ändern. Geringfügige Größenabweichungen der beiden Spannungen voneinander beeinflussen aber dio Dunkelschaltung viel mehr als die Hellschaltung, da die Differenz zweier nahezu gleich großer Größen bei Änderung einer der beiden Größen prozentual viel rascher zunimmt als die Summe. Die resultierende Spannung wird daher bei der Dunkelschaltung unter Umständen gar nicht auf den Wert Null zurückgehen, so daß man überhaupt nicht zum Parallelschalten kommt. Aber auch bei vollkommener Gleichheit der Spannungen wird das Parallelschalten bei Dunkelschaltung, namentlich bei unruhig laufenden Maschinen, wesentlich größere Schwierigkeiten machen, da der für die Dunkelschaltung maßgebende Nulldurchgang der Schwebungskurve sehr rasch erfolgt. Das Einlegen des Hauptschalters ist daher nur während einer sehr kurzen Zcit möglich. Hierdurch wird der Maschinenwärter leicht ängstlich, so daß er entweder zu früh oder zu spät einschaltet. Der bei der Hellschaltung maßgebende Durchgang der Schwebungskurve durch den Höchstwert erfolgt dagegen verhältnismäßig langsam. Man kann ihn daher viel leichter verfolgen und kann demgemäß auch vicl ruhiger und sicherer einschalten.

Meßtechnisch ist die Dunkelschaltung insofern im Nachteil, als alle Anzeigeapparate, also sowohl die Lampen als auch die Spannungsmesser, in der Nähe der Spannung Null besonders unempfindlich sind, während sie in der Nähe der Höchstspannung besonders genau anzeigen. Diese Eigentümlichkeit der Spannungsanzeigeapparate führt daher eher zu dem Schlusse, daß die Hellschaltung vorzuziehen sei, da nicht die Größenverhältnisse, sondern die Meßmöglichkeiten der rorkommenden Spannungen für die praktische Anwendung einer Schaltung ausschlaggebend sein müssen. Nan hat diesen meßtechnischen Nachteil der Dunkelschaltung in der neuesten Zeit dadurch beseitigt, daß man die Nullspannungsmesser so ausführt, daß sie gerade in der Nähe Skirl, Parallelschalten. 2. Aufl. 
des Nullpunktes eine besonders große Empfindlichkeit aufweisen (vgl. S. 34).

Hinsichtlich der Betriebssicherheit ist die Hellschaltung der Dunkelschaltung unbedingt überlegen, da die bei der Hellschaltung auftretende, in den Meßgeräten wirksame Summenspannung eben nur bei tatsächlichem Vorhandensein der beiden Teilspannungen nuftreten kann, während die bei der Dunkelschaltung maßgebende Spannung Null auch durch eine Störung, z. B. durch Draht. bruch in der Lampe oder im Spannungsmesser, vorgetäuscht werden kann. Eine Störung in der Meßeinrichtung wird daher bei der Dunkelschaltung leicht zu einer Fehlschaltung Anla $\$$ geben.

Schaltungstechnisch ist die Dunkelschaltung hinsichtlich ihrer Einfachheit und Übersichtlichkeit der Hellschaltung überlegen. Bei der Dunkelschaltung entstehen alle Schaltungen durch einfaches Vergleichen von Punkten gleichen Potentials, während bei der Hellschaltung stets Vertauschungen, also Leitungsüberkreuzungen, erforderlich sind, die die Ubbersichtlichkeit der Schaltung erschweren und die wahlweise Vergleichung von mehr als zwei Spannungen unmöglich machen. Hierzu kommt noch der Umstand, daB sich bei den indirekten Schaltungen mit MeBwandlern infolge der durch die Leitungsüberkreuzungen bedingten Polvertauschungen eine cinwandfreie Erdung der Meßwandler nicht durchführen läßt.

Die vorstehenden Erwägungen führen zu dem Schlusse, daß meßtechnisch die Hellschaltung und die Dunkelschaltung bei Verwendung entsprechender Meßinstrumente annähernd gleichwertig sind. Jedenfalls dürften die Vorteile der einen oder der anderen Schaltungsart nicht so ausschlaggebend sein, daß sie für die Wahl bestimmend sind. Hinsichtlich der Betriebssicherheit gebührt dagegen der Hellschaltung unbedingt der Vorzug, um so mehr, als von der Betriebssicherheit der Parallelschalteinrichtung das Wohl und Wehe des Kraftwerkes abhängt. Schaltungstechnisch gebührt hingegen wiederum der Dunkelschaltung der Vorzug, da diese eine für alle Maschinen vollkommen gleichartige Schaltung und cine einwandfreie Erdung der Meßwandler er möglicht. Dieser letztgenannte Umstand hat, da gerade dic Erdung durch dic Sicherheitsvorschriften des Verbandes deutscher Elektrotechniker verlangt wird, bisher dazu geführt, daß die Dunkelschaltung in den meisten Fällen angewendet wird. Durch 
die vom Verfasser angegebene neue Umkehrschaltung sind die schaltungstechnischen Schwierigkeiten, die zur Wahl der Dunkelschaltung führten, beseitigt. Die parallel zu schaltenden Maschinen werden hierbei stets entsprechend der Dunkelschaltung gleichpolig verbunden, während dic am Umkehrtransformator liegenden Meßgeräte in Hellschaltung arbeiten. Die Umkehrschaltung vereinigt also die schaltungstechnischen Vorteile der Dunkelschaltung mit den betriebstechnischen Vorteilen der Hellschaltung. Man kann bei Verwendung des Umkehrtransformators auch die Dunkel. schaltung mit der Hellschaltung zu einer gemischten Schaltung verbinden, indem man einen Teil der Meßgeräte in Dunkelschaltung und einen anderen in Hellschaltung arbeiten läßt. Auch hierbei wird dic Betriebssicherheit der Parallelschalteinrichtung gegenüber der reinen Dunkelschaltung wesentlich vergrößert, ganz abgesehen von den Vorteilen, die daraus erwachsen, daß man durch die festliegende Maschinenschaltung nicht an eine bestimmte Schaltung der Apparate gebunden ist. Man kann daher bei schwierigen Betriebsverhältnissen gegebenenfalls die verschiedenen Meßgeräte gleichzeitig ausprobieren und sich auf diese Weise eine allen Anforderungen entsprechende Parallelschalteinrichtung zusammenstellen. 


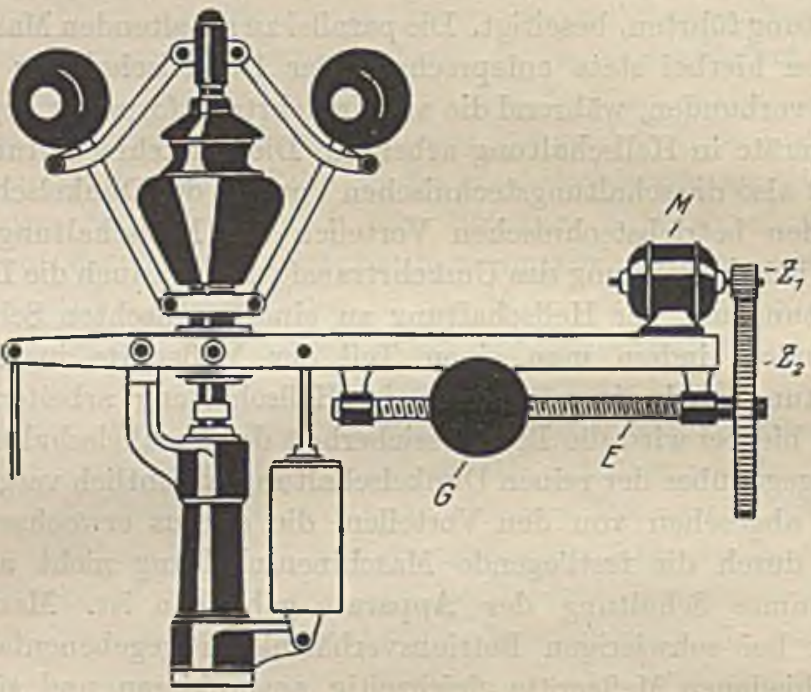

Bild 14. Der Gleichstrom-Motor $M$ treibt über das Zahnradrorgelege $Z_{1} Z_{2}$ die Einstellspindel $E$ an und bewegt auf diese Weise das Laufgewicht $G$. Die Verschiebung des Laufgewichts nach rechts hat cine Beschleunigung der Antriebsmaschine zur Folge, während eine Verschiebung nach links einer Verzögerung der Antriebsmaschine entspricht.

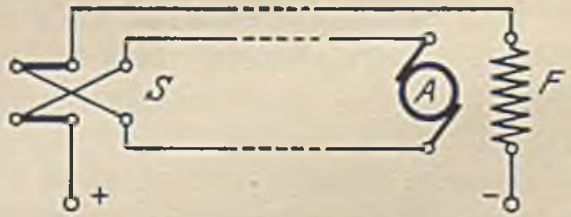

Bild 15. Durch den an der Bedienungsschalttafel angebrachten Umschalter $S$ wird der Einstellmotor des Regulators umgesteuert.

Tufel 6. Elektrische EInstellrorrichtung für den Regulator der Antriebsmaschine. 


\section{Die technischen Hilfsmittel zum Parallelschalten.}

\section{a. Elektrische Einstellyorrichtung fïr den Regulator der Antriebsmaschine.}

Da die Leistung einer parallel geschalteten Wechselstrommaschine nur von der Antriebsseite aus geregelt werden kann (vgl. S. 3), ist es erforderlich, auch die Antriebsmaschine von der Schalttafel aus zu regeln. Die hierzu erforderliche Änderung der Regulatorbelastung wird zweckmäßig durch die nachstehend beschriebene elektrische Einstellvorrichtung vorgenommen. Diese Vorrichtung ist auch für den Vorgang des Parallelschaltens sehr zweckdienlich, da sich mit ihr die Drehzahl der zuzuschaltenden Maschine von der Schalttafel aus sehr genau auf die Netzfrequenz einstellen läßt.

Die von den SSW. gebaute Einstellvorrichtung ist in Bild 14 schematisch dargestellt. Sie besteht im wesentlichen aus einem kleinen Elektromotor $M$, der über ein Vorgelege $Z_{1} Z_{2}$ die Einstellspindel $E$ des Regulators antreibt und auf diese Weise das Regulatorgewicht $G$ verschiebt. Bei Verschiebung des Gewichts nach rechts wird der Regulator belastet und damit der Dampfzutritt zur Maschine vergrößert, während bei Verschicbung nach links eine Entlastung des Regulators und damit eine Abdrosselung der Dampfzufuhr eintritt. An der Schalttafel, von der aus die Ferneinstellung erfolgen soll, wird lediglich ein Hebelumschalter angebracht, durch den der Motor $M$ in der einen oder anderen Drehrichtung eingeschaltet wird, je nachdem ob die Leistung der Kraftmaschine vergrößert oder verkleinert werden soll (vgl. Bild 15). Der Umschalter ist so eingerichtet, daß er beim Loslassen selbsttätig in die Ausschaltstellung zurückkehrt. Um ein Einschalten des Motors nach erreichter Endstellung des Laufgewichts zu verhüten, ist an dem Vorgelege ein Endausschalter angebracht, der den Motor selbsttätig in der Endstellung ausschaltet.

\section{b. Frequenzmesser.}

Nachdem die Antriebsmaschine in Betrieb gesetzt ist, wird mittels der vorherbeschriebenen Einstellvorrichtung dic Frequenz des zuzuschaltenden Generators genau eingestellt. Zur Messung der Frequenz benutzt man einen elektrischen Frequenzmesser. 


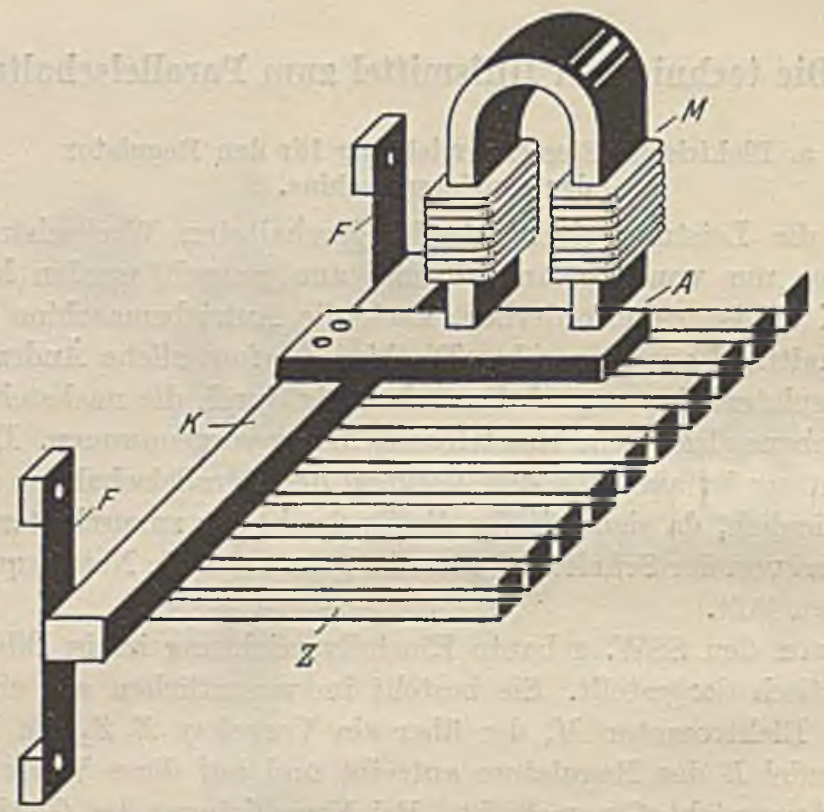

Bild 16. Indirekte Erregung der Zungen. (Bauart Siemens.)

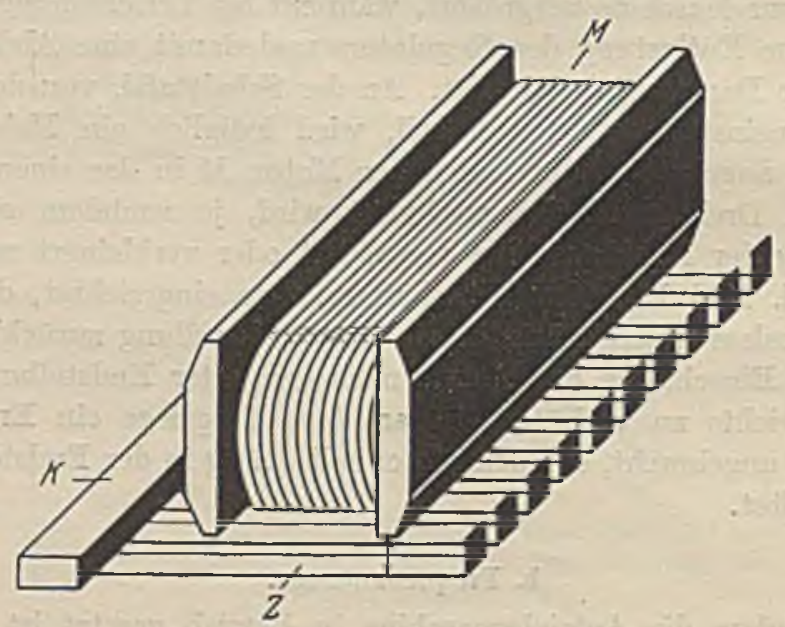

Bild 17. Direkte Erregung der Zungen. (Bauart Hartmann \& Braun.)

Talel 7. Meßwerke der Zungenfrequenzmesser. 
Das Meßwerk der Zungenfrequenzmesser beruht auf dem Resonanzprinzip. Es besteht aus einer Reihe Federn, sog. Zungen, dio auf verschiedene Eigenschwingungszahlen mechanisch abgestimmt sind. Die Zungen stehen unter der Einwirkung eines Elektromagneten. Wird dieser von dem $\mathrm{zu}$ untersuchenden Wechselstrom durchflossen, so geraten diejenigen Zungen, deren Eigenschwingungszahl mit der Frequenz des Wechselstromes übereinstimmt, infolge der Resonanzwirkung in heftige Schwingungen, so $\mathrm{da} \beta$ ein deutlich sichtbares Schwingungsbild entsteht. Dio übrigen Zungen, deren Eigenschwingungszahl von der Frequenz des Wechselstromes abweicht, schwingen nur ganz leicht mit, so daß sie praktisch in Ruhe erscheinen. Die verschiedenen Bauformen der Frequenzmesser unterscheiden sich durch die Art der Übertragung der Schwingungen des Wechselstromes auf die Zungen. Bei den Frequenzmessern von S. \& H. sind sämtliche Zungen $Z$ auf einem gemeinsamen, auf Federn gelagerten Steg, dem Zungenkamm $K$, befestigt, wie es Bild 16 zeigt. Der Zungenkamm trägt einen Anker $A$, der einem feststehenden Elektromagneten $M$ gegenübersteht. Bei Erregung des Elektromagneten werden daher die elektrischen Schwingungen zunächst auf den Zungenkamm und von diesem auf die Federn übertragen. Bei den in Bild 17 dargestellten Frequenzmessern von Hartmann \& Braun werden dagegen die Zungen unmittelbar elektrisch erregt. Die Stahlzungen $Z$ werden hierbei auf einer festen Unterlage $K$ angebracht und durch einen längs der ganzen Zungenreihe verlaufenden Elektromagneten $M$ in Schwingungen versetzt. Das Schwingungsbild ist bei beiden Bauformen das gleiche.

Um die Frequenz der zuzuschaltenden Maschine bequem mit der Netzfrequenz vergleichen zu können, vereinigt man zweckmäßig den Maschinenfrequenzmesser mit dem Netzfrequenzmesser zu einem Doppelfrequenzmesser. Bei diesem liegen die beiden Skalen dicht übereinander, wie es die Bilder auf S. 48 und 50 zeigen. Entsprechend dem Vorgang beim Parallelschalten wird das Schwingungsbild der zuzuschaltenden Maschine auf der oberen Skala wandern, während das zur Netzfrequenz gehörige Schwingungsbild auf der unteren Skala feststeht. Die Drehzahl der zuzuschaltenden Maschine wird dann solange geregelt, bis die beiden Schwingungsbilder genau übereinanderstehen. 


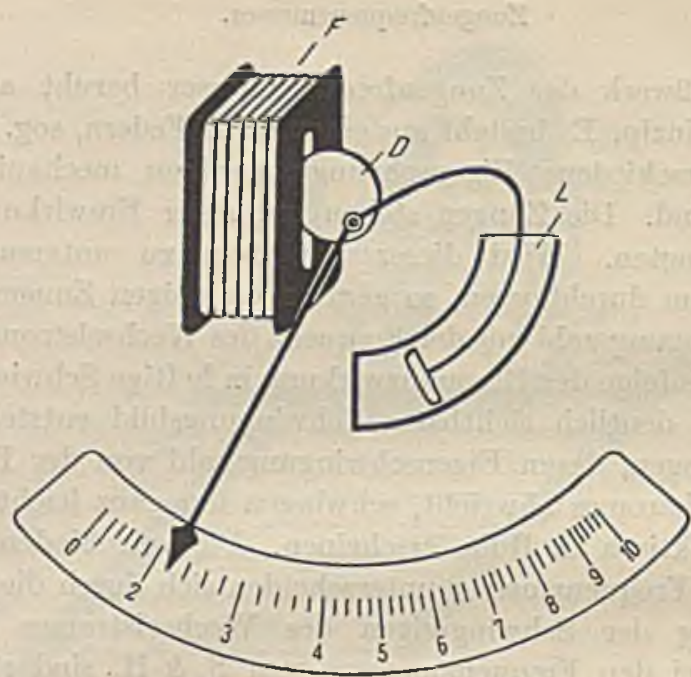

Bild 18. Flachspultype. Das Eisenblättchen $D$ wird in die Feldspule $F$ hineingezogen und erzeugt so den Zeigerausschlag. Als Gegenkraft dient eine Spiralfeder.

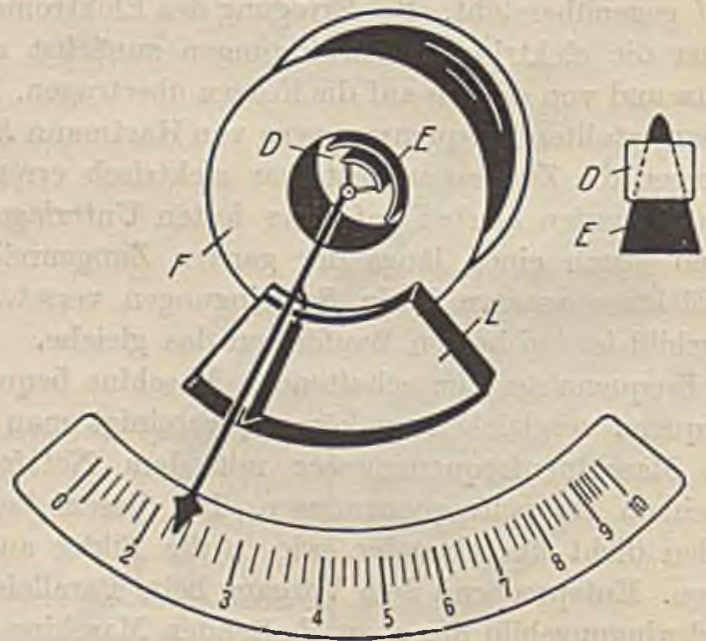

Bild 19. Rundspultype. Innerhalb der Feldspule $F$ ist ein feststehendes Eisenstückchen $E$ angebracht. das auf das bewegliche Eisenstückehen $D$ absto Bend wirkt. Die Bewegungen werden durch die Luftdämpfung $L$ gedümpft.

Tafel 8. Meßwerke der Spannungsmesser. 


\section{c. Spannungsmesser.}

Nachdem die Frequenz der zuzuschaltenden Maschine richtig eingestellt ist, wird auch ihre Spannung auf den der Netzspannung entsprechenden Wert gebracht. Zum Vergleichen der beiden Spannungen werden bei den Parallelschalteinrichtungen meist besondere Spannungsmesser vorgesehen.

Als Meßwerk für diese Instrumente wird vorzugsweise das Dreheisen-Meßwerk benutzt. Dieses besteht im wesentlichen aus einem drehbar gelagerten Eisenstückchen und einer vom zu messenden Strome durchflossener Feldspule. Unter der Einwirkung des in der Feldspule fließenden Stromes wird das Eisenstückchen in den Hohlraum der Spule hineingezogen bzw. in ihm bewegt. Je nach der Form der Feldspule unterscheidet man Flachspul- und Rundspul-Meßwerke. Bei den von S. \& H. gebauten Flachspul-Instrumenten wird ein kleines herzförmiges Eisenstückchen benutzt, das exzentrisch auf der Zeigerachse gelagert ist und in den Hohlraum einer seitlich angeordneten Spule hineingezogen wird (vgl. Bild 18 auf Tafel 8). Bei den von Hartmann \& Braun, der Allgem. Elektrizitäts-Gesellschaft u. a. gebauten Rundspul-Instrumenten wird cine zur Zeigerachse konzentrische, kreisförmige Spule benutzt, an deren Innenwand noch ein festes Eisenstückchen angebracht ist (vgl. Bild 19). Das auf der Zeigerachse sitzende bewegliche Eisenstückchen wird darm in gleichem Sinne magnetisiert wie das feststehende. Es besteht daher cine abstoßende Kraft, deren Größc durch dic Formgebung der Eisenstückchen bedingt ist. Als Gegenkraft dient moistens eine Spiralfeder.

Um das Vergleichen der beiden Spannungen zu erleichtern, vereinigt man zweckmäßig den Maschinenspannungsmesser und den Netzspannungsmesser zu einem Doppelspannungsmesser. Die beiden Meßwerke werden hierbei hintereinander angeordnet, so daß die Zeiger über einer Skala spielen (vgl. Tafel 12). Um mit nur einer Skalenteilung auszukommen, führt $\mathrm{S}$. \& $\mathrm{H}$. die Eichung so aus, daß der Nullpunkt und der der Normalspannung entsprechende Teilstrich für beide Meßwerke übercinstimmen. Die weiteren Teilstriche werden dann als Mittelwerte eingezeichnet. Auf diese Weise wird es erreicht, daß die Angaben der beiden Meßwerke für die praktisch allein in Frage kommende Normalspannung genau übereinstimmen. Die ctwaigen Abweichungen 
treten nur bei den übrigen, weniger wichtigen Skalenteilen auf und werden überdies durch die Mittelwertseinzeichnung halbiert, so daß auch hier eine praktisch vollkommen ausreichende $\mathrm{MeB}$. genauigkeit crzielt wird. Da der Zeiger für die Netzspannung im normalen Betriebe stets auf einem bestimmten, der normalen Betriebsspannung entsprechenden Werte steht und der Zeiger der hinzuzuschaltenden Maschine auf diesen Wert eingestellt werden soll, wird der Zeiger für die Netzspannung als rote bewegliche Kennmarke ausgebildet, wie aus Tafel 12 ebenfalls ersichtlich ist. Die zuzuschaltende Maschine wird dann stets so erregt, daß der Zeiger des oberen Meßwerkes über dieser Kennmarko cinspielt.

\section{d. Phasonlampen.}

Nachdem die zuzuschaltende Maschine auf die richtige Frequenz und Spannung gebracht ist, muß noch die Phasengleichheit zwischen Maschine und Netz hergestellt werden. Hierzu kann man einfache Glühlampen benutzen, die man, wie auf S. 8 u. 10 beschrieben, entweder als Phasenlampen für Dunkelschaltung oder für Hellschaltung schaltet. Für ein exaktes Parallelschalten, namentlich bei größeren Maschinen, reichen jedoch die Phasenlampen wegen der geringen erreichbaren Meßgenauigkeit nicht aus. Bei der Dunkelschaltung verlischt die Phasenlampe, bevor die Spannung wirklich gleich Null wird; bei der Hellschaltung ist zwar das Lichtmaximum leicht erkennbar, jedoch stört hier die Blendwirkung der Lampe. Man verwendet daher die Phasenlampen nicht als selbständige, sondern nur als ergänzende Meßmittel zu anderen, genaueren Meßgeräten. Sie haben dann im wesentlichen den Zweck, durch ihr Aufleuchten bzw. Verlöschen dem Beobachter anzuzeigen, daß die Parallelschalteinrichtung ordnungsgemäß arbcitet.

Um zu vermeiden, daß die Phasenlampen schon vorher verlöschen, ehe die Spannung gleich Null wird, verwendet die Firma Hartmann \& Braun für die Dunkelschaltung eine besondere Schaltweise, bei der die Lampen durch eine konstante Spannung bis zum Beginn des Leuchtens vorbelastet werden. Die Wirkungsweise dieser Schaltung ist aus Bild 20 ersichtlich. An Stelle der sonst üblichen zwei Phasenlampen ( $v$ gl. Bild 3) werden hierbei vier Phasenlampen verwendet, die durch einen regelbaren Wider- 
stand $R$, ähnlich wie bei einer Brückenschaltung, miteinander verbunden sind. Die Lampen $P_{1}$ und $P_{2}$ liegen über diesen Widerstand an der konstanten Netzspannung, während die Lampen $P_{3}$ und $P_{4}$, ebenfalls über diesen Widerstand, an der konstanten

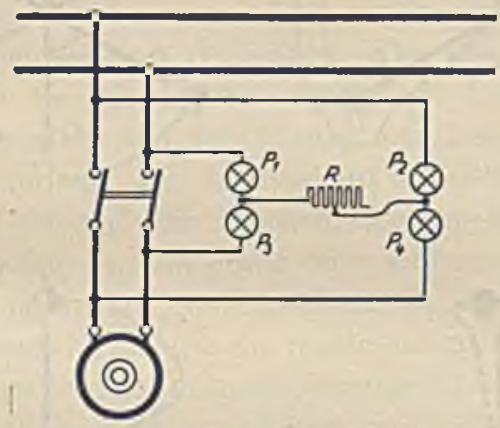

Bild 20.

Maschinenspannung liegen. Der Widerstand $R$ wird so eingestellt, daß alle vier Lampen bei Phasengleichheit durch die von den beiden Spannungen gelieferten Vorbelastungsströme bis zum Beginn des Leuchtens gebracht werden. Während des Vorganges der Parallelschaltung werden dann die Lampen ebenso wie bei der normalen Schaltung aufleuchten und verlöschen, jedoch fällt der Zeitpunkt des Verlöschens dann mit dem Zeitpunkt der Phasengleichheit zusammen.

Um die Blendwirkung der hellaufleuchtenden Lampen zu beseitigen und es von vornherein kenntlich zu machen, ob sie in Hell- oder Dunkelschaltung arbeiten, baut S. \& H. die Phasenlampen in Gehäuse mit einer Transparentscheibe ein. Bei Hellschaltung wird die Transparentscheibe so ausgeführt, daß beim Aufleuchten der Lampe das Signal ,Achtung" sichtbar wird und den Maschinenwärter darauf aufmerksam macht, daß der Augenblick des Parallelschaltens nahe ist. Bei Dunkelschaltung wird dagegen die Scheibe so eingerichtet, daß beim Aufleuchten der Lampe das-Warnungssignal ,Nicht schalten" erscheint. Man kann jedoch auch bei Dunkelschaltung an Stelle des Warnungssignales ein Achtungssignal erhalten, wenn man die vom Verfasser vorgeschlagene Umkehrschaltung (vgl. S. 12) verwendet. Man erreicht hierdurch eine wesentlich größere Betriebssicherheit. 

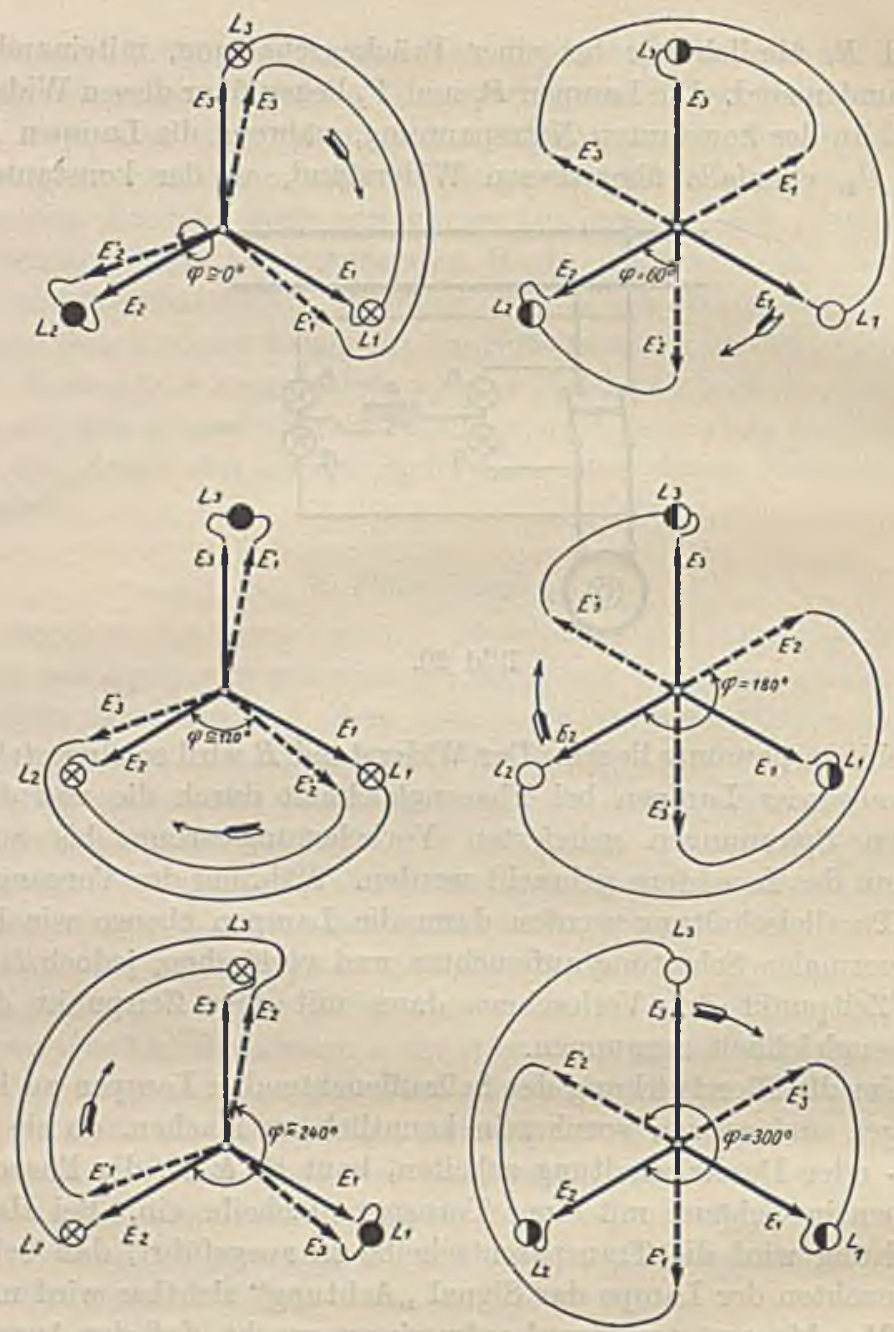

Es bedeutet:

Bild 21 bis 26.

- Lampe ist dunkel $\left(E_{t}=0\right)$.

- zunchmende Lichtstärke $\left(E_{l}=E_{p}=0,58 E\right)$.

$\otimes$ mittlere Helligkeit $\left(E_{1}=1,73 E_{p}=E\right)$.

größte Lichtstärke $\left(E_{l}=2 E_{p}=1,15 E\right)$.

- abnehmende Lichtstärke $\left(E_{l}=E_{p}=0,58 E\right)$.

Tafel 9. Darstellung der Spannungs- und Lichtrerhiltnisse am Dreilampen-Apparat. 


\section{c. Lampenapparato.}

Zum Parallelschalten von Drehstrom-Maschinen kann man an Stelle der einfachen Phasenlampen für Hell- oder Dunkelschaltung auch die auf $\mathrm{S}$. 15 beschriebene Umlaufschaltung benutzen, bei der die Lampen nicht gleichzeitig, sondern nacheinander aufleuchten und verlöschen. Der auf dieser Schaltung beruhende, von Dr. Michalke angegebene Lampenapparat besteht in seiner einfachsten Ausführung aus drei im Dreieck angeordneten Glühlampen. Der Lichtschein wandert hierbei jo nachdem, ob die Drehzahl der zuzuschaltenden Maschine zu hoch oder zu niedrig ist, in dem einen oder anderen Sinne im Kreise herum, so daß man ohne weiteres sehen kam, in welchem Sinne die zuzuschaltende Maschine zu regeln ist.

Die Arbeitsweise des Lampenapparates ist aus dem Diagramm auf S. 28 er'sichtlich. In diesem Diagramm stellt der Stern $E_{1} E_{2} E_{3}$ die Sternspannungen des Netzes und $E_{1}^{\prime} E_{2}^{\prime} E_{3}^{\prime}$ die entsprechenden Sternspannungen der zuzuschaltenden Maschine dar. Die Phasenverschiebung zwischen diesen beiden Spannungssystemen ist durch den Winkel $\varphi$ bezeichnet. Um eine leichte Übersichtlichkeit $\mathbf{z u}$ erzielen, sind die Phasenlampen $L_{1} L_{2} L_{3}$ unmittelbar an die entsprechenden Spannungsvektoren angeschlossen. Die an den einzelnen Lampen auftretende Spannung ist dann durch die Resultierende der mit den Lampen verbundenen Vektoren gegeben. Allerdings muß hierbei beachtet werden, daß die Vektoren in bezug auf den Stromkreis gegeneinander geschaltet sind. Die resultierende Spannung ist demgemäß nicht die Summe, sondern die geometrische Differenz der beiden Einzelspannungen. Hiernach ergeben sich für das erste Diagrammbild die folgenden Verhältnisse. Die beiden räumlich gleichgerichteten, gleichgroßen Vektoren $E_{2}$ und $E_{2}^{\prime}$ heben einander auf, da sie im Stromkreis gegencinander geschaltet sind. Ihre Resultierende ist demgemäß gleich Null. Die Lampe $L_{2}$ verlischt daher. Die an der Lampe $L_{1}$ auftretende Spannung ergibt sich als Resultierende der Spannungen $E_{1}$ und $E_{3}^{\prime}$ dadurch, daß man den einen Vektor $E_{3}^{\prime}$ um $180^{\circ}$ herumklappt und ihn geometrisch $\mathrm{zu} E_{1}$ addiert. Da die Spannungen $E_{1}$ und $-E_{3}^{\prime}$ um $60^{\circ}$ verschoben sind, beträgt die Resultierende $1,73 \cdot E p=E$, die Lampe $L_{1}$ brennt daher mit der vollen Netzspannung. In analoger Weise ergibt sich die Spamnung an der Lampe $L_{3}$ als Resultierende der Spannungen $E_{3}$ und $E_{1}^{\prime}$. Die 
Lampe $L_{3}$ brennt daher ebenfalls mit voller Netzspannung. Im nächsten Bild erreicht die Spannung der Lampe $L_{1}$ ihren Höchstwert $2 \cdot E p=1,15 E$, während die Lampen $L_{2}$ und $L_{3}$ nur noch mit annähernd der halben Netzspannung brennen. Hierbei ist jedoch zu beachten, daß die Lichtstärke der Lampe $L_{3}$ im Abnehmen begriffen ist und die der Lampe $L_{2}$ zunimmt. In den folgenden Diagrammbildern sind die Verhältnisse für die verschiedenen, zeitlich nacheinander auftretenden Phasenverschiebungen $\varphi$ durchgeführt und ergeben an Hand der eingezeichneten Lampenbilder deutlich das Wandern des Lichtscheins bei der Änderung der Phasenverschiebung. Der Drehsinn des Lichtscheins hängt davon ab, ob sich der Spannungsstern $E_{1}^{\prime} E_{2}^{\prime} E_{3}^{\prime}$ in dem einen oder in dem anderen Sinne gegen den Spannungsstern $E_{1} E_{2} E_{3}$ verschiebt, d. h., ob die zuzuschaltende Maschine zu langsam oder zu schnell läuft. Hat die zuzuschaltende Maschine die synchrone Drehzahl erreicht, so bleibt der Lichtschein stehen. Die Verteilung der Spannung auf die drei Lampen hängt hierbei lediglich von der Phasenverschiebung der zu vergleichenden Spannungen ab. Wird diese Phasenverschiebung gleich Null, ist also Phasengleichheit erreicht, so verlischt die in Dunkelschaltung liegende Lampe $L_{2}$, während die beiden anderen Lampen mit der verketteten Spannung brennen. Es ist demgemäß bei dem Lampenapparat nicht nur das Wandern des Lichtscheins und sein Stehenbleiben zu beachten, sondern der Lichtschein muß in einer ganz bestimmten Lage stehenbleiben, wenn Phasengleichheit erreicht ist. Um die hieraus entstehende Unsicherheit zu vermeiden, schaltet man meistens parallel zu der als Phasenlampe geschalteten Glühlampe einen Nullspannungsmesser und liest an diesem die Phasengleichheit ab. Der Lampenapparat wird dann lediglich zum Einstellen auf die synchrone Drehzahl benutzt. Er bietet hierbei den Vorteil, daß er auf größere Entfernungen abgelesen werden kann, so daß eine besondere Befehlsübertragung von der Schaltbühne zur Maschine nicht erforderlich ist.

Bei der besseren Ausführung des Lampenapparates werden an Stelle der drei Lampen sechs Glühlampen verwendet, von denen immer je zwei gegenüberliegende parallel geschaltet sind. Die Lampen werden hierbei durch das Gehäuse verdeckt und sind um einen konischen Reflektor angeordnet, wie Bild 28 zeigt. Die Wirkungsweise dieses Apparates ergibt sich ohne weiteres aus 

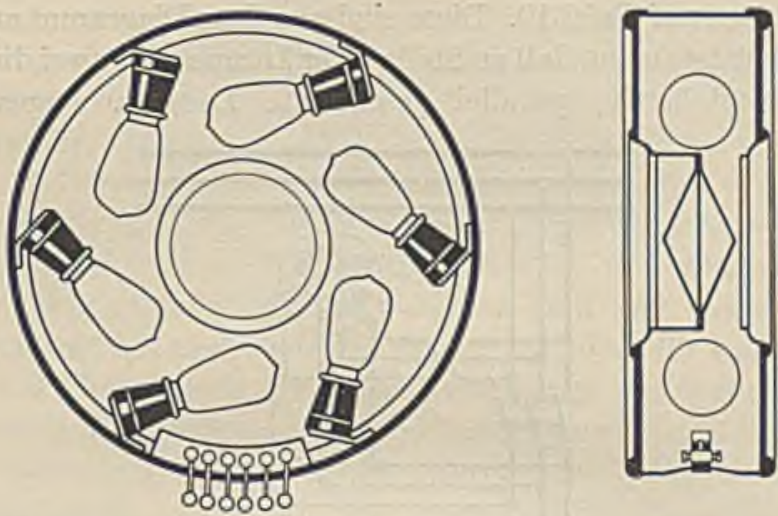

Bild 27 und 28. Innenansicht des Sechslampen-Apparates. Die verdeckt angeordneten Lampen erzeugen auf dem konischen Reflektor einen rotierenden Schattenstrich.

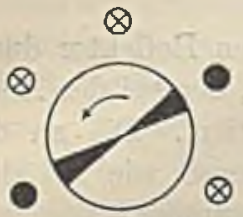

$\otimes$

D

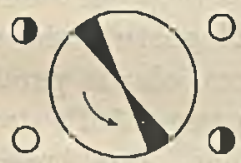

(1)

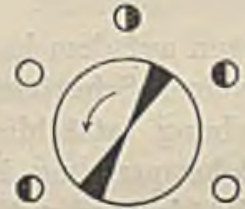

(1)

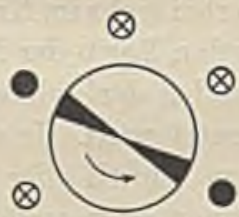

Q

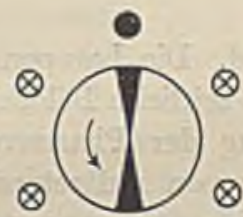

○

O

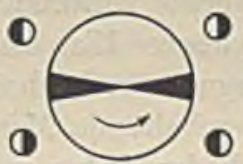

O

Bild 29 bis 34. Darstellung der Lichtrerhältnisse im Sechslampen-

Es bedeutet: Apparat.
- Lampe ist dunkel $\left(E_{l}=0\right)$.
- zunehmende Lichtstärke $\left(E_{l}=E_{p}=0,58 E\right)$.
$\otimes$ mittlere Helligkeit $\left(E_{l}=1,73 E_{p}=E\right)$.
größ to Lichtstãrke $\left(E_{l}=2 E_{p}=1,15 E\right)$.
O abnehmendo Lichtstärke $\left(E_{l}=E_{p}=0,58 E\right)$.

Tufel 10. Bauart und Wirkungsweise des Sechslampen-Apparates. 
den Bildern auf Tafel 10. Diese sind aus dem Diagramm auf S. 28 dadurch entstanden, daß an Stelle einer Lampe stets zwei diametral gegenüberstehende, parallel geschaltete Lampen eingezeichnet

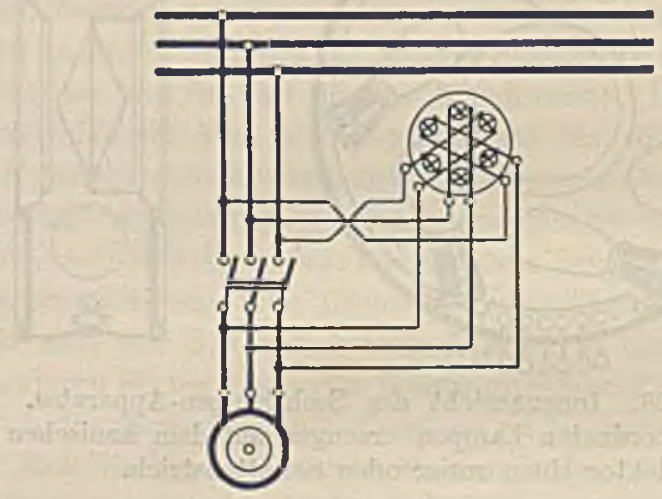

Bild 35.

sind. Die Lampen erzeugen auf dem konischen Reflektor durch die seitliche Beleuchtung einen Schattenstrich, der bei der Änderung der Phasenverschiebung tatsächlich rotiert. Die an den Lampen auftretende Höchstspannung ist ebenso wie bei dem Dreilampenapparat das 1,15 fache der Netzspannung.

Die AEG. baut einen Apparat mit umlaufendem Zeiger, der in gleicher Weise wie der Lampenapparat geschaltet ist. An Stelle der Lampen sind hierbei sechs im Kreise angçordnete Elektromagnete verwendet. Vor den Polen der Magnete liegt ein dünner, drehbarer Eisenanker, der bei der zyklischen Magnetisierung der Nagnete in Drehung versetzt wird. Je nachdem, ob die parallel zu schaltende Maschine zu schnell oder zu langsam läuft, dreht sich der Anker in dem einen oder anderen Sinne. Der Apparat unterscheidet sich von den in Abschnitt i) beschriebenen Synchronoskopen mit umlaufendem Zeiger dadurch, daß der Anker bei Phasengleichheit nicht in einer bestimmten Stellung stehenbleibt. Zur Feststellung der Phasengleichheit ist daher stets noch ein besonderer Apparat erforderlich.

\section{P. Nullspannungsmesser.}

Da die Phasenlampen nur eine verhältnismäßig rohe Schätzung der Spannung gestatten, benutzt man zweckmäßig zur genaueren 
Messung noch einen besonderen Spannungsmesser, den man parallel zu den Phasenlampen anschließt. Bei der Dunkelschaltung muß dieser Spannungsmesser so gebaut sein, daß er in der Nähe des Nullpunktes genaue Ablesungen gestattet, $d$. h. seine Skala muß am Anfang weit auseinandergezogen sein. Man nennt einen so gebauten Spannungsmesser einen Nullspannungsmesser. Beim Parallelschaltensch wankt der Zeiger entsprechend den Schwebungen der Spannungskurve dauernd zwischen Null und einem Höchstwert hin und her und bleibt bei Phasengleichheit für cinen Augenblick auf Null stehen. Der dem Höchstwert der Spannung entsprechende Meßbereich des Nullspannungsmessers muß je nach der Schaltung für die doppelte Netzspannung, für die doppelte Sternspannung, oder für die doppelte Sekundärspannung der Meßwandler bemessen sein.

Bei der Beurteilung der Wirkungsweise des Nullspannungsmessers muß man beachten, daß dieser nur die an den Schalterkontakten auftretenden Spannungsdifferenzen anzeigt, ganz unabhängig davon, ob diese durch Phasen- oder Spannungsverschiedenheiten verursacht werden. Wie auf S. 3 bereits gesagt wurde, kommt es jedoch beim Parallelschalten in erster Linie darauf an, die durch Phasenverschiedenheiten verursachten Spannungsdifferenzen zu vermeiden, da diese die gefährlichen, wattleistenden Ausgleichströme zur Folge haben. Um es zu erreichen, $d a ß$ der Nullspannungsmesser nur diese gefährlichen Spannungsdifferenzen anzeigt, muß die Spannung der zuzuschaltenden Maschine so geregelt werden, da $\beta$ sie genau die gleiche Größe wie die Netzspannung erhält. Es genügt keineswegs, die Spannung der zuzuschaltenden Maschine nur annähernd gleich der Netzspannung zu machen, da die im Nullspannungsmesser auftretende Spannungsdifferenz die Differenz zweier nahezu gleicher Größen darstellt und demgemäß bei Verschiedenheiten sehr rasch anwüchst. Beachtet man dies nicht, so wird es vorkommen, daß der Nullspannungsmesser überhaupt nicht auf Null zurückgeht, so daß man es nicht wagen kann, die Parallelschaltung zu vollziehen.

Die Ausführung eines guten Nullspannungsmessers wird dadurch besonders erschwert, da $B$ alle Wechselstrom-Spannungsmesser eine nahezu quadratische Skalenteilung aufweisen. Die Ablesung ist daher gerade in der Nähe des Nullpunktes, der für

Skirl, Parallelschalten. 2. Aufl. 


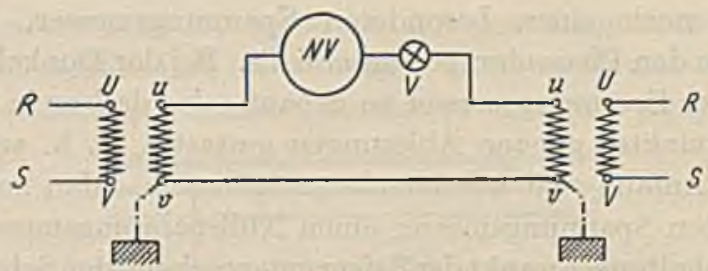

Bild 36. Äußere Schnltung eines Nullspannungemessers mit Vorschaltlampe.

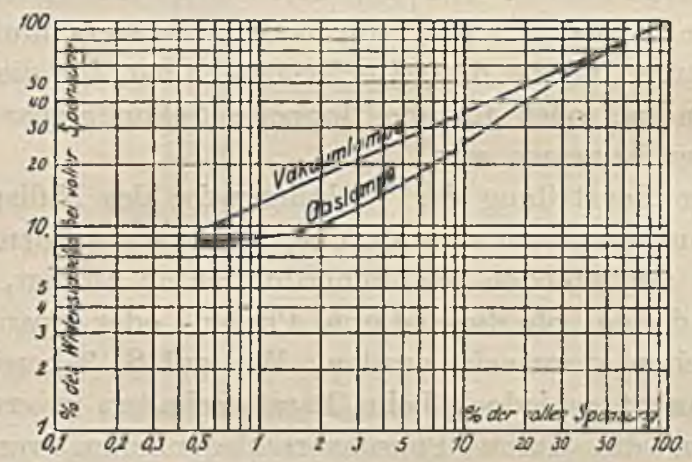

Bild 37. Widerstandsānderung der Forschaltiampe als Funktion der Spannung in logarithmischer Dar. stellung.

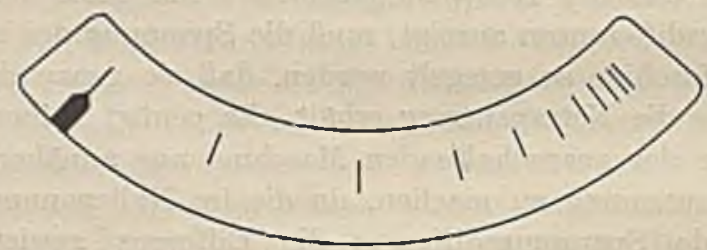

Bild 38. Skalenteilung eines Nullspannungsmessers mit Dreheisen-Meßwerk und Vorschaltlampe. Durch den veränderlichen Vorwiderstand sind die Skalenteile am Anfang sehr weit auseinandergozogen, so $\mathrm{da} B$ eine besonders große Anfangsempfindlichkeit dos Instrumentes erreicht ist. Der Wert eines Skalentejles ist ein Zehntel des Skalenendwertes.

Tafel 11. Nullspannungsmesser mit Vorschaltlampe. 
Dunkelschaltung einzig und allein in Frage kommt, besonders ungenau. Man versuchte diesen Nachteil dadurch zu mildern, daB man die Anfangsteilung der Instrumente besonders weit auseinanderzog und den Endausschlag nicht bei der auftretenden Höchstspannung, sondern etwa bei der Hälfte oder cinem Drittel der Höchstspannung eintreten ließ. Hierdurch ergab sich aber der Nachteil, daß der Zeiger von der Hälfte bzw. einem Drittel der Spannung bis herauf zur vollen Spannung am oberen Anschlag fest anlag, so daß man die Änderungen der Spannung nicht mehr dauernd verfolgen konnte.

Bei den von Dr. Keinath angegebenen Nullspannungsmessern von $\mathrm{S}$. \& $\mathrm{H}$. ist eine besonders weite Anfangsteilung dadurch erreicht, daß an Stelle des üblichen Vorwiderstandes aus Manganin ein solcher aus einem Metall mit hohem Temperaturkoeffizienten verwendet wird. Als veränderlicher Vorwiderstand dient hierbei eine Metalldrahtlampe. Die luftleeren und die gasgefüllten Lampen verhalten sich, wie das Kurvenbild auf Tafel 11 zeigt, etwas verschieden. Bei der luftleeren Drahtlampe nimmt der Widerstand vom kalten bis zum warmen Zustande um etwa den zehnfachen Betrag des Anfangswertes zu. Bei den gasgefüllten Lampen ist die Widerstandszunahme bei der Endtemperatur entsprechend der höheren Temperatur des Glühfadens etwas größer (etwa zwölffach) und andererseits bei kleineren Temperaturen infolge anderer Wärmeabfuhrverhältnisse etwas kleiner. Durch Verwendung einer solchen Lampe als Vorwiderstand wird die Anfangsteilung des Spannungsmessers ganz bedeutend verbessert, und zwar um so mehr, als der Instrumentwiderstand gegen den Lampenwiderstand $\mathrm{zu}$ vernachlässigen ist. Bei kleinen Spannungen ist dann der Widerstand der Glühlampe und somit der Vorwiderstand des Spannungsmessers sehr klein, so daß das Instrument einen großen Ausschlag gibt. Bei höheren Spannungen wächst mit der Spannung der Widerstand der Glühlampe; der Vorwiderstand wird also immer größer und somit wächst der Zeigerausschlag nur langsam an. Der Skalenverlauf eines Nullspannungsmessers mit Dreheisen-Meßwerk und gasgefüllter Vorschaltlampe ist aus Bild 38 ersichtlich. Der Wert eines Skalenteils ist hierbei durchweg ein Zehntel des Skalenendwertes. Der für das Parallelschalten maBgebende Nullpunkt der Skala ist durch eine rote Kennmarke besonders hervorgehoben. Dem Vorteil der besonders günstigen 


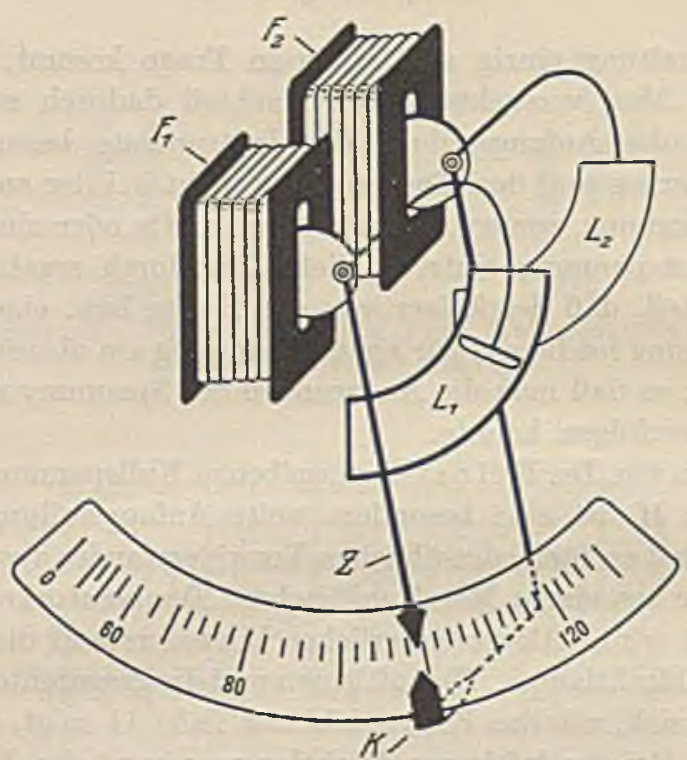

Bild 39. Der Summenspannungsmesser besitzt zwei Dreheisen-Meßwerke, wie sie auf Tafel 8 beschrieben sind. Der Zeiger deg hinteren Meßwerkes greift von unten um die Skala herum und ist als Kennmarke ausgebildet. Bei Phasengleichheit muß der Zeiger des rorderen MeBwerkes über dieser Kennmarke einspielen.

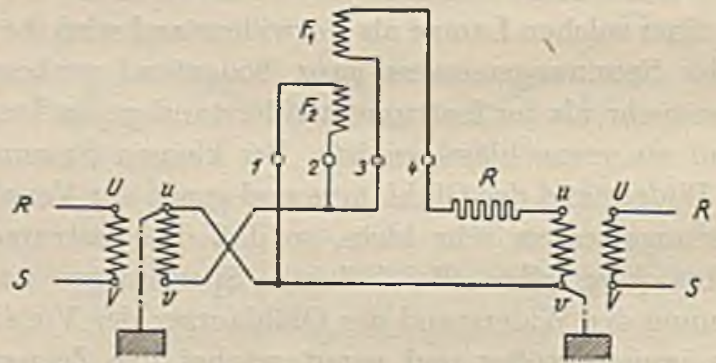

Bild 40. Äußere Schaltung. Das hintere Meßwerk nit der Feldspule $F_{2}$ ist unmittelbar an die Netzspannung angeschlossen, während das rordere 3FeBwerk mit einem Vorwiderstand $R$ an der Summenspannung liegt.

Talel 12. MeBwerk und Schaltung des Summenspannungsmessers. 
Skalenteilung steht indessen gegenüber, da $B$ die Betriebssicherheit des Instrumentes durch den immerhin empfindlichen Faden der Glihhlampe verringert wird. Es empfiehlt sich daher, als Kontrolle parallel zum Nullspannungsmesser eine in Hellschaltung liegende Phasenlampe mit Umkehrtransformator anzuschließen (vgl. Bild 10 auf S. 13).

Etwa beschädigte Vorschaltlampen können ohne weiteres gegen neue Lampen ausgewechselt werden. Eine Neueichung des Meßinstruments wird dadurch nicht erforderlich, da der Widerstand von allen Lampen der gleichen Type annähernd der gleiche ist. Die etwa vorkommenden Abweichungen in der Größenordnung von $2 \%$ sind für einen Nullspannungsmesser belanglos.

\section{g. Summenspannungsmesser.}

Bei der Hellschaltung wird zur Vergrößerung der Meßgenauigkeit ebenfalls ein Spannungsmesser benutzt, der parallel zu den Phasenlampen angeschlossen wird. Da bei der Hellschaltung stets die Summe der Netzspannung und der Naschinenspannung bestimmend ist, wurde für den hierbei verwendeten Spannungsmesser die neue Bezeichnung "Summenspannungsmesser" gewählt. Beim Parallelschalten schwankt der Zeiger des Summenspannungsmessers entsprechend den Schwebungen der Spanmungskurve dauernd zwischen Null und einem Höchstwert hin und her und bleibt bei Phasengleichheit für einen Augenblick auf dem Höchstwert stehen. Die Skala des Summenspannungsmessers muß daher in der Nähe des Höchstwertes besonders fein unterteilt sein. Der dem Höchstwert der Spannung entsprechende Meßbereich des Summenspannungsmessers muß je nach der Schaltung für die doppelte Netzspannung, für die doppelte Sternspannung oder für die doppelte Sekundärspannung der Meßwandler ausreichen.

Für die Wirkungstreise des Summenspannungsmessers gelten ähnliche Gesichtspunkte, wie sie bei der Besprechung des Nullspannungsmessers gebracht wurden. Der Summenspannungsmesser zeigt lediglich die Summe der Netzspannung und der Spannung der zuzuschaltenden Maschine an, ganz unabhängig davon, ob ihr Wert durch verschiedene Phase oder verschicdene Größe der beiden verglichenen Spannungen erreicht wurde. Er trennt also ebenfalls nicht die Phasenverschiedenheiten von den 
Spannungsverschiedenheiten. Ist die Spannung der zuzuschaltenden Maschine zu hoch, so zeigt der Summenspannungsmesser den für die Parallelschaltung maßgebenden Ausschlag gleich der doppelten Netzspannung schon an, bevor die Phasengleichheit erreicht ist, während andererseits bei zu kleiner Spannung der zuruschaltenden Maschine der erforderliche Wert überhaupt nicht erreicht wird. Aber eine einfache Utberlegung ergibt, daß die Spannungsverschiedenheiten hierbei nicht die Rolle spielen lkönnen wie beim Nullspannungsmesser, denn die Summe zweier nahezu gleich großer Größen wird durch eine geringe Änderung eines der beiden Summanden nur unwesentlich beeinflußt. Man kann daher hierbei unter Umständen auf einen besonderen Doppelspannungsmesser verzichten und die Spannung der zuzuschaltenden Maschine lediglich nach dem Höchstausschlag des Summenspannungsmessers einstellen.

Seiner Bauart nach ist der Summenspannungsmesser ein gewöhnlicher Spannungsmesser mit Dreheisen-Meßwerk. Er ist gekennzeichnet durch eine auf dem Werte der doppelten Netzspannung stehende Kennmarke, auf die der Zeiger beim Parallelschalten einspielen muß. Bei der einfachsten Ausführung wird die Kennmarke so angebracht, daß sie von Hand auf den jeweiligen Wert der doppelten Netzspannung eingestellt werden kann. Bei der besseren Ausführung dagegen exfolgt die Einstellung der Kennmarke selbsttätig. Man verwendet hierzu den im vorigen Abschnitt beschriebenen Doppelspannungsmesser (vgl. Tafel 12). Das hintere Meßwerk, dessen Zeiger als bewegliche Kennmarke ausgeführt ist, wird unmittelbar an die Netzspannung bzw. an die Sekundärseite des an der Netzspannung liegenden Spannungswandlers angeschlossen. Die Kennmarke wird dann stets auf den jeweiligen Wert der Netzspannung einspielen. Das vordere Meßwerk, das als Summenspannungsmesser geschaltet wird, erhält einen äußeren Vorwiderstand, durch den der Meßbereich verdoppelt wird. Der Zeiger dieses Meßwerkes steht dann bei der doppelten Netzspannung direkt über der beweglichen Kennmarke.

\section{h. Synchronoskop mit schwingendem Zeiger.}

Während die Null- und Summenspannungsmesser lediglich die Differenz bzw. die Summe der parallel zu schaltenden Spannungen anzeigen, ganz gleichgültig, ob diese durch verschiedene Größe 


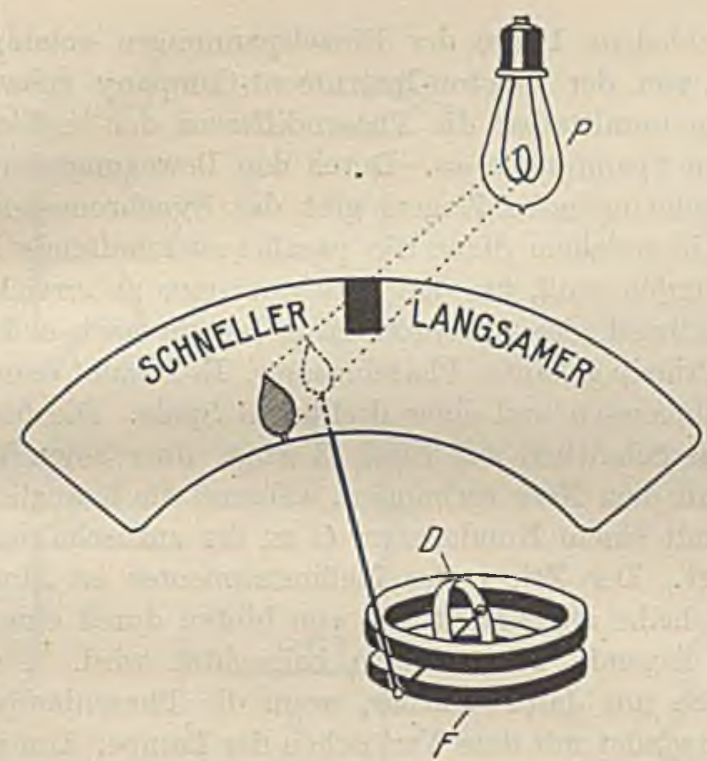

Bild 41. Das Meßwerk ist cin nach elektrodynamischem Prinzip gebauter Phasenmesser und besteht demnach aus ciner feststehenden Feldspule $F$ und der Drehspule $D$. Der hinter der Transparentscheibe befindliche Zciger wird durch die Phasenlampe beleuchtet, so daß auf der Skala sein Schattenbild entsteht.

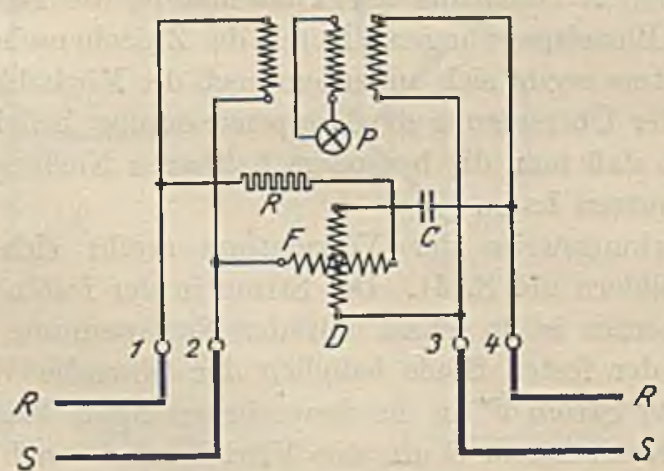

Bild 42. Schaltung des Synchronoskops (vgl. S. 40).

Tafel 13. Weston-Synchronoskop mit schwingendem Zeiger, 
oder verschiedene Phase der Einzelspannungen entstanden ist, zeigt das von der Weston-Instrument-Company gebaute Synchronoskop unmittelbar die Phasendifferenz der beiden zu vergleichenden Spannungen an. Durch den Bewegungssinn des hin und her schwingenden Zeigers gibt das Synchronoskop gleichzeitig an, in welchem Sinne die parallel zu schaltende Maschine geregelt werden muß, um den Synchronismus zu erreichen.

Das Meßwerl des Synchronoskops ist ein nach elektrodynamischem Prinzip gebauter Phasenmesser. Es besteht demnach aus einer feststehenden und einer drehbaren Spule. Die feste Spule ist, wie das Schaltbild auf Tafel 13 zeigt, über einen Vorwiderstand $R$ mit dem Netz verbunden, während die bewegliche Spule in Reihe mit einem Kondensator $C$ an der zuzuschaltenden Maschine liegt. Der Zeiger des Meßinstrumentes ist hinter einer Milchglasscheibe angebracht, die von hinten durch eine in Hellschaltung liegende Phasenlampe beleuchtet wird. Der Zeiger ist demnach nur dann sichtbar, wenn die Phasenlampe brennt und verschwindet mit dem Verlöschen der Lampe. Um unmittelbare Verbindungen zwischen dem Netz und der zuzuschaltenden Maschine zu vermeiden, ist die Phasenlampe nicht direkt angeschlossen, sondern liegt an der Sekundärwickelung eines dreischenkeligen Transformators, der cinerseits vom Netz und andererseits von der zuzuschaltenden Maschine erregt wird. Die in der Sekundärwickelung erzeugte Spannung ist demnach ebenso wie bei der direkten Schaltung der Phasenlampe die Resultierende der beiden Einzelspannungen. Durch die Zwischenschaltung des Transformators ergibt sich außerdem noch die Möglichleeit, durch Änderung der Utbersetzung die Lampenspannung beliebig herabzusetzen, so daß man die besonders haltbaren Niederspannungslampen benutzen kann.

Die Wirkungsweise der Vorrichtung ergibt sich aus den Diagrammbildern auf S. 41. Der Strom in der festen Spule des Meßinstrumentes ist in Phase mit der Netzspannung $E$, da im Stromkreis der festen Spule lediglich der Ohmsche Widerstand $R$ liegt. Der Strom $J^{\prime}$ in der beweglichen Spule dagegen wird durch den Kondensator $C$ um eine Viertelperiode nach vorn verschoben; er eilt daher stets um $90^{\circ}$ vor der Spannung $E^{\prime}$ der zuzuschaltenden Maschine voraus. Im ersten Diagrammbild ist die Spannung $E^{\prime}$ um einen Winkel $\varphi=270^{\circ}$ ror der Netzspannung 

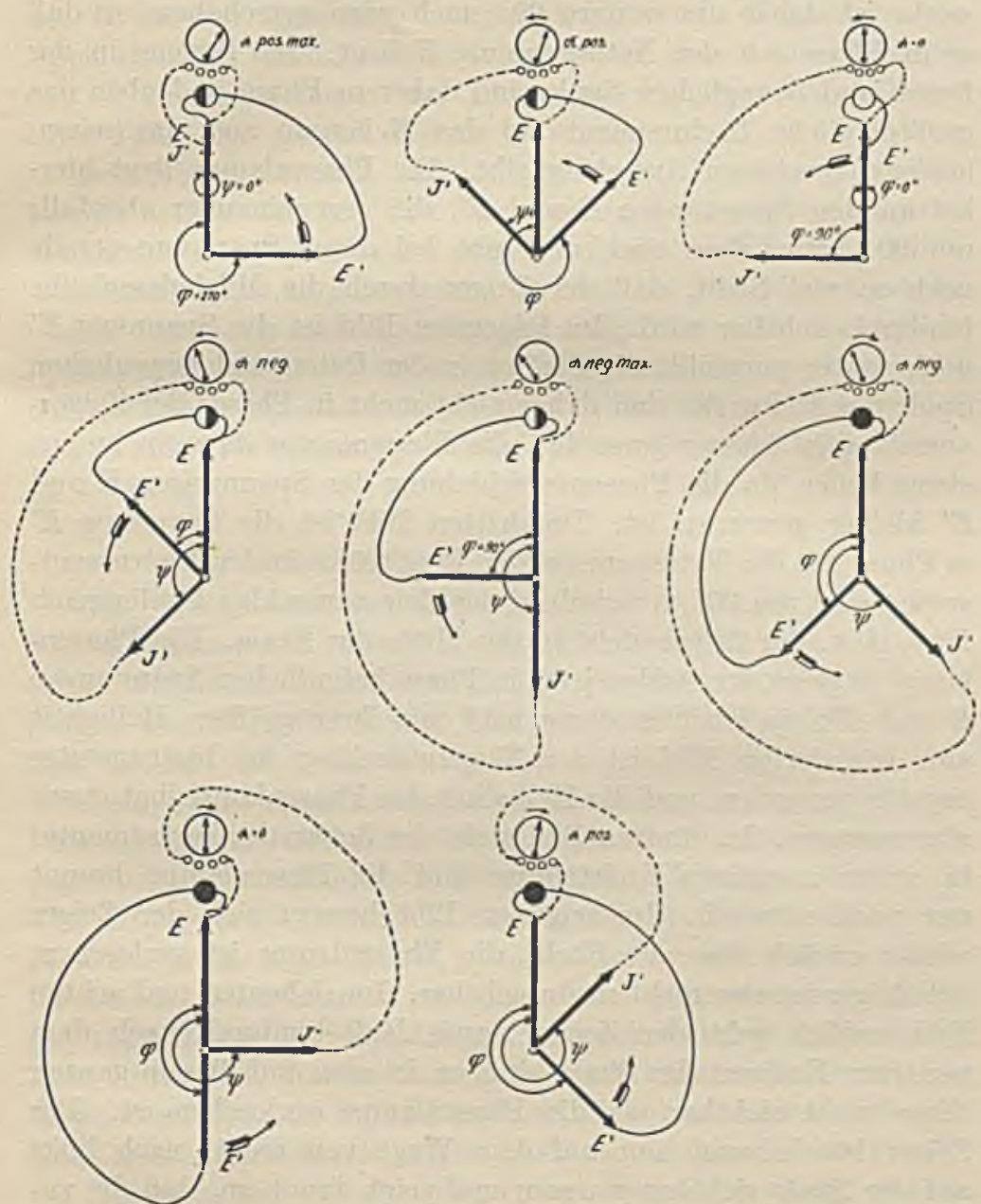

Es bedeutet:

Bild 43 bis 50 .
- - Phasenlampe ist dunkel,
$0=$ volle Lichtstärke,
$0=$ zunehmendo Lichtstärke,
$0=$ abnehmende Lichtstärke.

Tafol 14. Graphische Darstellung der Arbeitsweise des WestonSynchronoskops. 
$E$ vorausgeeilt. Der Strom $J^{\prime}$ in der bewoglichen Spule des Meß. werks ist daher um weitere $90^{\circ}$ nach vorn verschoben, so daß er in Phase mit der Netzspannung $E$ liegt. Die Ströme in der festen und beweglichen Spule sind daher in Phase und üben das größtmögliche Drehmoment auf das Meßorgan aus, das seinen höchsten positiven Ausschlag gibt. Die Phasenlampe liegt hierbei an den Spannungen $E$ und $E^{\prime}$, die gegeneinander ebenfalls um $90^{\circ}$ verschoben sind, und gibt bei diescr Spannung gerado noch so viel Licht, daß der Zeiger durch die Milchglasscheibe hindurch sichtbar wird. Im folgenden Bild ist die Spannung $E^{\prime}$ noch weiter vorgeeilt, die Ströme in der festen und beweglichen Spule des Meßwerks sind daher nicht mehr in Phase; der Zeigerausschlag ist kleiner geworden, die Phasenlampe dagegen brennt etwas heller, da die Phasenverschiebung der Spannungen $E$ und $E^{\prime}$ kleiner geworden ist. Im dritten Bild ist die Spannung $E^{\prime}$ in Phase mit der Netzspannung $E$. Die Ströme in den Instrumentspulen sind um $90^{\circ}$ verschoben, der Zcigerausschlag ist demnach Null, d. h. der Zeiger steht in der Mitte der Skala. Die Phasenlampe liegt an den beiden jetzt in Phase befindlichen Spannungen $E$ und $E^{\prime}$; sie leuchtet demgemäß mit ihrer größten Helligkeit auf. Im vierten Bild ist der Zeigerausschlag des Instrumentes negativ geworden, und die Helligkeit der Phasenlampe hat etwas abgenommen. Im fünften Bild steht der Zeiger des Instrumentes in seiner negativen Endstellung und die Phasenlampe brennt nur noch schwach. Im sechsten Bild bewegt sich der Zeiger wieder zurück über die Slaala, die Phasenlampe ist verloschen, der Zeiger ist also nicht mehr sichtbar. Im siebenten und achten Bild endlich geht der Zeiger durch Null hindurch nach dem positiven Endwert der Skala hinüber, ist aber auf diesem ganzen Wege nicht sichtbar, da die Phasenlampe verloschen ist. Der Zeiger ist demnach nur auf dem Wege von rechts nach links auf der Skala sichtbar gewesen und zeigt damit an, daß die zuzuschaltende Maschine schneller läuft, als es der synchronen Drehzahl entspricht. Würde die zuzuschaltende Maschine langsamer laufen, so würde sich das Spiel umkehren, d. h. der Zeiger würde nur auf dem Wege ron links nach rechts sichtbar sein. Bei Synchronismus endlich bleibt der Zeiger in der im dritten Diagrammbild dargestellten Weise mitten in der vollbeleuchteten Skala stehen. Das Westor-Synchronoskop hat den Vorteil, da B 
seine Angaben innerhalb weiter Grenzen von der Frequenz und der Größe der Spannungen unabhängig sind. Gegenüber den Apparaten mit umlaufendem Zeiger hat es jedoch den Nachteil, daß es die Änderungen der Phasenverschiebung nur während eines Teiles der ganzen Periode anzeigt.

Der Eigenverbrauch des Synchronoskops einschließlich der Lampe beträgt bei 110 Volt nur etwa 15 Voltampere von jeder Maschine. Der Apparat ist ohne weiteres für Ein- und Mehrphasenmaschinen zu gebrauchen, da auch bei Mehrphasenmaschinen die Schaltung meistens einphasig ausgeführt wird. Für höhere Spannungen als 110 Volt kann das Synchronoskop wegen des dazugehörigen Spezialtransformators nicht eingerichtet werden und ist daher für höhere Spannungen stets mit Spannungswandlern zu benutzen.

\section{i. Synchronoskope mit umlaufendem Zeiger.}

Die Synchronoskope mit umlaufendem Zeiger sind zum Parallelschalten von Drehstrom-Maschinen bestimmt. Je nachdem, ob die Drehzahl der zuzuschaltenden Maschine zu hoch oder zu niedrig ist, läuft der Zeiger in dem einen oder anderen Drehsinn dauernd im Kreise herum und zeigt auf diese Weise an, in welchem Sinne die zuzuschaltende Maschine zu regeln ist. Bei Phasengleich heit bleibt der Zciger in der durch eine Kennmarke besonders hervorgehobenen senkrechten Stellung stehen. Da diese Apparate die Bewegungsvorgänge der parallel zu schaltenden Maschine genau wiedergeben, gestatten sie ein sehr sicheres Parallelschalten. Man kann es mit ihnen ohne weiteres erreichen, daß die zuzuschaltende Maschine sofort nach dem Parallelschalten Last aufnimmt, indem man nur dann einschaltet, wenn der Zeiger des Apparates in der Richtung „Zu schnell" die der Phasengleichheit entsprechende Marke passiert. Da die Wirkungsweise der Apparate nur von den Phasenverhältnissen abhängt, werden sie von etwaigen Spannungsverschiedenheiten nicht beeinflußt.

Die Anordnung des von S. \& H. hergestellten Synchronoskops ist auf Tafel 15 dargestellt. Der Apparat besteht aus einem feststehenden, aus Fisenblechen aufgebauten Ständer $P$ mit zwei bewickelten Polen und einem zwischen diesen Polen drehbar angeordneten Läufer $A$ mit einer normalen Drehstromwickelung. Die Ständerwickelung wird einphasig an die Spannung der zuzu- 


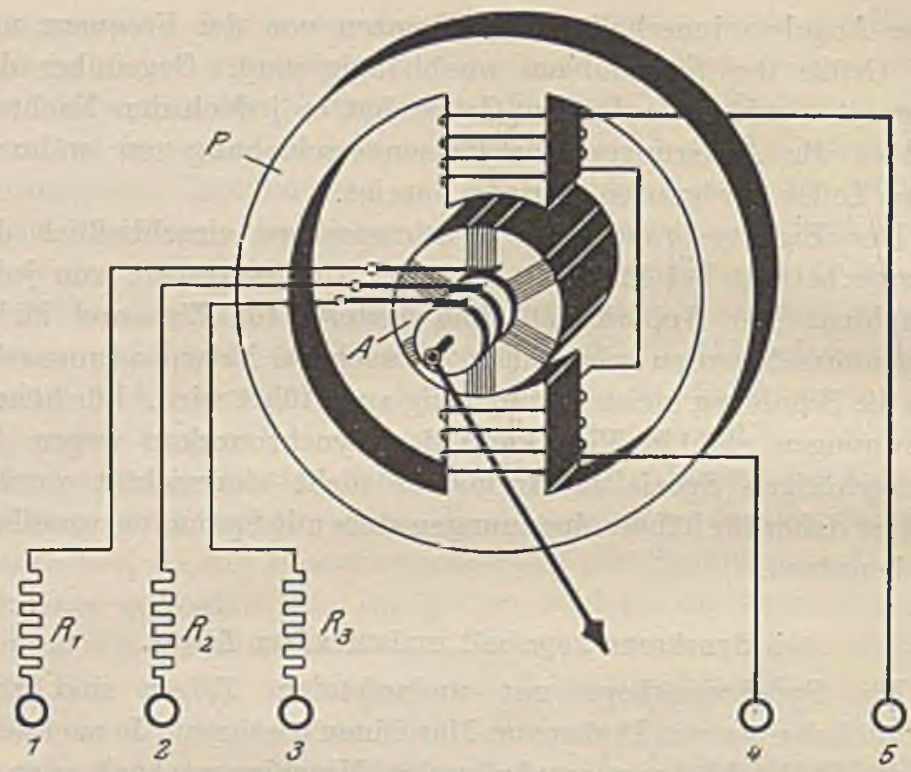

Bild 51. Der zweipolige Stānder $P$ trägt eine Einphasenwickelung. während der Liufer $A$ dreiphasig gewickelt ist. Infolge der Wechsclwirkung zwisehen dem Drehfeld des Läufers und dem Einphasenfeld des Ständers drcht sich der Läufer bei Frequenzverschicdenheit je nach dem Sinne der Verschiedenheit dauernd in der einen oder anderen Richtung. Bei Frequenzgleichheit bleibt der Lãufer in einer der jewciligen Phasenverschiebung entsprechenden Stellung stehen. Bei Phasengleichheit spielt der Zeiger über einor Kennmarko ein.

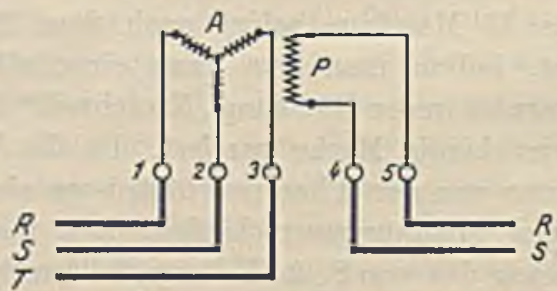

Bild 52. Äußere Schaltung. Die dreiphasige Lāuferwickelung $A$ wird stets an die bereits laufende Maschine angeschlossen, während die einphasige Ständerwickelung $P$ an der zuzuschaltenden Maschine liegt.

Tafel 15. Siemens-Synchronoskop mit umlaufendem Zeiger. 
schaltenden Maschine angelegt, während die Läuferwickelung dreiphasig an das Netz bzw. die bereits laufende Maschine angeschlossen wird. Im Ständer entsteht dann ein einphasiges Wechselfeld, das mit der Frequenz der zuzuschaltenden Maschine zwischen den feststehenden Polen des Ständers hin und her schwingt, während im Läufer ein Drehfeld erzeugt wird, das mit der Netzfrequenz umläuft. Die Wirkungsweise des Apparates beruht auf der Wechselwirkung zwischen diesen beiden Feldern. Der Läufer wird sich stets so einstellen, daß der Vektor seines Drehfeldes in dem Augenblick in die Richtung des Wechselfeldes fällt, in dem dieses seinen Höchstwert erreicht. Ist die Frequenz im Läufer etwas kleiner als die des Ständers, so wird der Vektor des Drehfeldes die der Richtung des Wechselfeldes entsprechende Stellung noch nicht ganz erreicht haben, wenn das Wechselfeld durch seinen Höchstwert hindurchgeht. Der Läufer wird sich daher um einen diesem Betrag entsprechenden Winkel nach vorwärts drehen. Beim nächsten Umlauf des Drehfeldes wird sich der Phasenunterschied noch weiter vergrößern, d. h. der Läufer wird sich noch weiter nach vorwärts drehen usf. Der Läufer wird also hierbei dauernd in einem bestimmten Sinne umlaufen. Ist die Frequenz im Läufer größer wie die in Ständer, so kehrt sich der Vorgang $u m$, so daß der Läufer in entgegengesetztem Sinne umläuft. Bei Frequenzgleichheit bleibt der Läufer in einer bestimmten Stellung stehen, die der beim Eintritt der Frequenzgleichheit gerade vorhandenen Phasenverschiebung $\mathbf{z w i s c h e n ~ S t a ̈ n d e r s t r o m ~}$ und Läuferstrom entspricht. Wird diese Phasenverschiebung gleich Null, d. h. tritt zu der Frequenzgleichheit auch noch die Phasengleichheit, so spielt der am Läufer angebrachte Zeiger auf einer bestimmten Marke der Skala ein.

Nach dem Vorstehenden läuft der Zeiger des Synchronoskops dauernd in dem einen oder anderen Sinne um, solange noch ein Frequenzunterschied zwischen dem Netz und der zuzuschaltenden Maschine besteht. Die Umlaufgeschwindigkeit des Zeigers hängt hierbei von der Größe des Frequenzunterschiedes ab. Wird dieser sehr groß, so wird der Zeiger so rasch umlaufen, daß eino sichere Ablesung nicht mehr möglich ist. Bei sehr großen Frequenzunterschieden kann es unter Umständen sogar vorkommen, daß der Zeiger infolge sekundärer Erscheinungen in verkehrtem Sinne umläuft. Um in jedem Falle eine sichere Ablesung zu bekommen, 


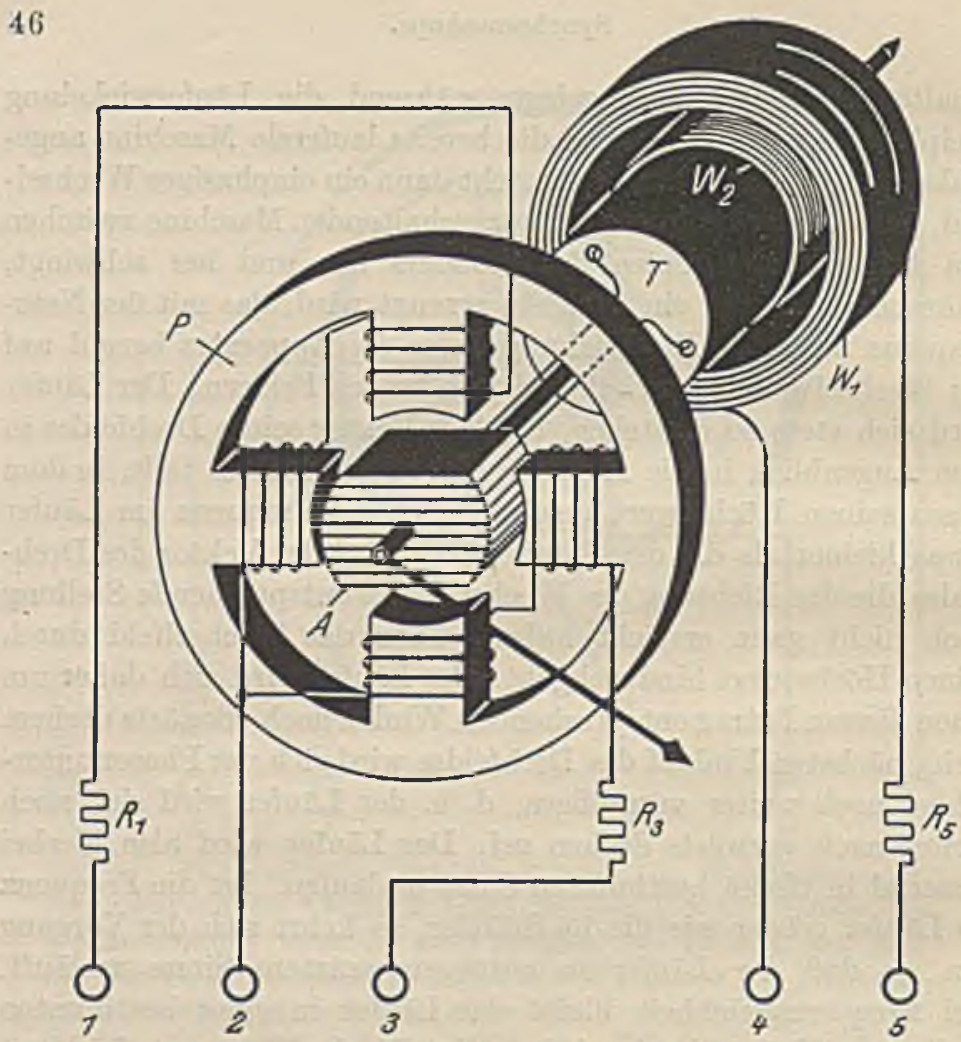

Bild 53. Das Charakteristische dieser Bauform ist die Art der Stromzuführung zum Läufer, die nicht in der sonst üblichen Weise durch Schleifringe und Bürsten, sondern induktiv erfolgt. $\mathrm{Zu}$ diesem $\mathrm{Zw}$ weke ist auf der verlängerten Achse des Lüufers $A$ eine mit dem Läufer umlaufende Sekundärwickelung $W_{2}$ eines Transformators angebracht (vgl. S. 47).

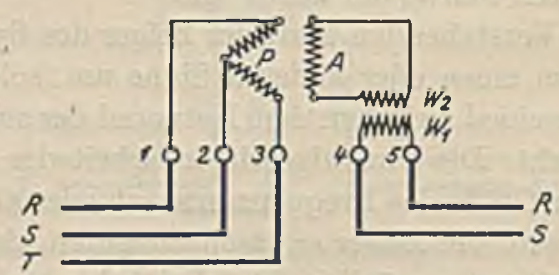

Bild 54. Dic Ständerwickelung $P$ wird stets an die laufende Maschine angeschlossen, wāhrend der Lüufer $A$ über den Transformator $W_{1} W_{2}$ an der zuzuschaltenden Maschine liegt.

Tafel 16. Synchronoskop von Hartmann \& Braun. 
soll das Synchronoskop stets erst dann eingeschaltet werden, wenn die Frequenz der zuzuschaltenden Maschine auf etwa $5 \%$ richtig eingestellt ist. Man benutzt für diese Grobeinstellung der Frequenz meist einen Doppelfrequenzmesser. Dieser bietet gleichzeitig noch den Vorteil, daß er auch bei größeren Frequenzunterschieden den Regelsinn der zuzuschaltenden Maschine angibt. Verzichtet man auf diesen Vorteil, so kann man gegebenenfalls auch mit den Phasenlampen auskommen, da der Zeiger des Synchronoskops im selben Rhythmus umläuft wie die Phasenlampen aufleuchten und verlöschen. Man wird das Synchronoskop hierbei erst dann einschalten, wenn die Lampen so langsam aufleuchten, daß man mit dem Auge bequem folgen kann. Der Eigenverbrauch des Synchronoskops ist sehr gering. Er beträgt z. B. bei 110 Volt in der Wechselstromwickelung etwa 0,1 Ampere und in der Drehstromwickelung etwa 0,18 Ampere. Infolge dieses geringen Stromverbrauchs ist es ohne weiteres zulässig, das Synchronoskop zusammen mit den Spannungsmessern an die gleichen Spannungswandler anzuschließen.

Bei dem von der Firma Hartmann \& Braun gebauten Synchronoskop ist der Ständer $P$ vierpolig ausgeführt und an zwei Phasen des Drehstromes angeschlossen, wie Tafel 16 zeigt. Der Läufer $A$ trägt eine einphasige Wickclung. Diese wird jedoch nicht, wie sonst üblich, durch Schleifringe und Bürsten, sondern induktiv gespeist. Hierzu dient ein besonderer Hilfstransformator. Die mit der Läuferwickelung verbundene Sekundärwickelung $W_{2}$ ist auf der Trommel $T$ angebracht, die ihrerseits auf der verlängerten Achse des Läufers sitzt und daher mit ihm umläuft. Die Primärwickelung $W_{1}$ des Transformators ist feststehend angeordnet. Bei der tatsächlichen Ausführung des Apparates sind die Wickelungen in Eisen eingebettet, so da $B$ eine günstige Transformatorwirkung erzielt wird. Durch die bei der Drehung mitgeführte Sekundärwickelung des Transformators wird jedoch in jedem Falle das Gewicht des beweglichen Organs erheblich vergrößert, so daß hierdurch der Vorteil der bürstenlosen Anordnung zum großen Teil wieder aufgehoben wird.

\section{k. Allgemeines über dio Auswahl der Meßgeräte.}

Die Doppelfrequenzmesser und die Doppelspannungsmesser werden bei allen Schaltungen in gleicher Weise angewendet, ganz 


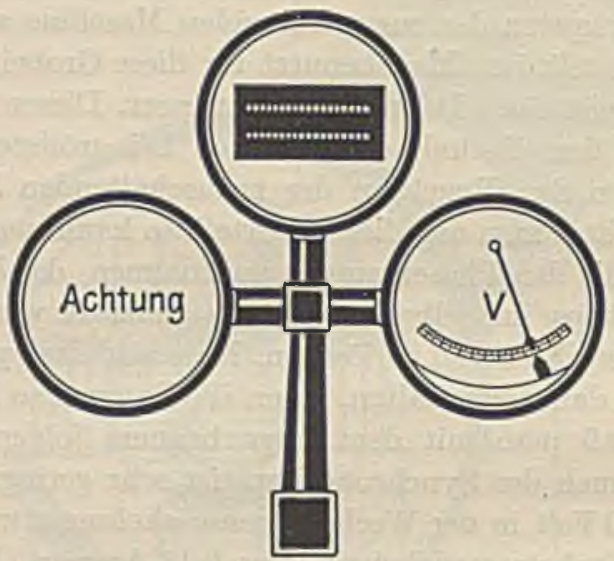

Bild 55. Meßeinrichtung mit Doppelfrequenzmesser, Summenspannungsmesser und einer in ein Gehäuse mit Transparentscheibe eingebøuten Phasenlampe.

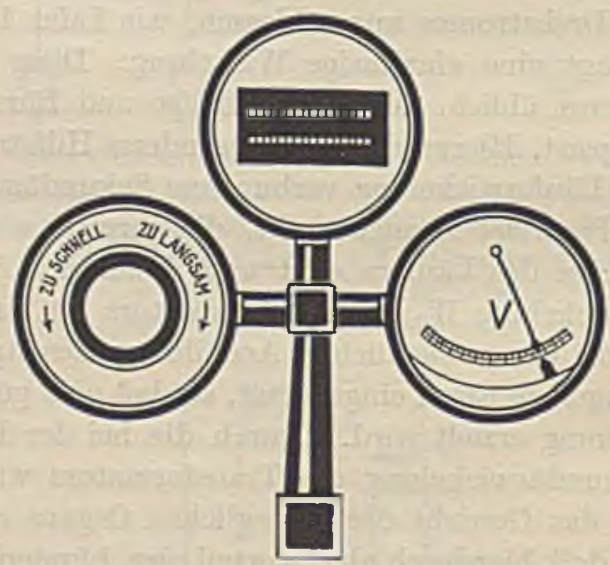

Bild 56. Meßcinrichtung mit Doppelfrequenzmesser, Summenspannungsmesser und Lampenapparat.

Tafel 17. Instrumentsätze für Hellschaltung. 
unabhängig davon, ob man sich für Hell- oder Dunkelschaltung entschieden hat (vgl. S. 16). In vielen Fällen wird man jedoch vor der Frage stehen, ob es richtiger ist, einen Null- bzw. Summenspannungsmesser oder ein Synchronoskop, einfache Phasenlampen oder einen Lampenapparat zu benutzen. Un die richtige Auswahl zu erleichtern, sollen daher im nachstehenden die grundsätzlichen Unterschiede dieser verschiedenen Neßgeräte noch einmal kurz gegenübergestellt werden.

Die Null- und Summenspannungsmesser zeigen nicht unmittelbar die Größe an, die man mit ihnen messen will. Man will die Phasenverschiedenheiten messen, die Instrumente zeigen aber lediglich eine Spanumgsdifferenz bzw. die Summe der parallelzuschaltenden Spannungen an, ganz unabhängig davon, ob diese Werte durch verschiedene Größe oder verschiedene Phase der Einzelspannungen entstanden sind. Wie wir auf $\mathrm{S}$. 3 geschen haben, verursachen Spannungsdifferenzen, die durch Größenverschiedenheiten der beiden Spannungen hervorgerufen werden, lediglich wattlose Ausgleichströme, die zwar Spannungsschwankungen verursachen könmen, aber sonst auf die Maschinen mechanisch keine wesentliche Rückwirkung ausüben. Dagegen üben die durch Phasenverschiedenheiten der Spannungen entstehenden Ausgleichströme eine sehr kräftige mechanische Rückwirkung auf die parallelzuschaltenden Maschinen aus, durch die die Maschinen und ihre Wickelungen sehr stark bennsprucht werden. Um Stöße beim Parallelschalten zu vermeiden, muß man daher in erster Linio die durch Phasenverschiedenheiten entstehenden Ausglcichströme möglichst klein halten. Da die Null- und die Summenspannungsmesser allein es nicht gestatten, die schädlichen Spannungen bzw. Ausgleichströme von den unschädlichen zu unterscheiden, muß man bei ihrer Verwendung stets noch einen Doppelspaunungsmesser genau beobachten und sich überzeugen, daß die beiden zu vergleichenden Spannungen genau die gleiche Größe haben.

Die Synchronoskope bieten demgegenüber den wesentlichen Vorteil, daß sie unabhängig von der Größe der Spannungen die zu messende Phasenverschiedenheit anzeigen. Wenn man mit einem Synchronoskop parallelschaltet, ist man daher stets sicher, daß man keine schädlichen Ausgleichströme bekommt, die die Maschinen ungünstig beanspruchen. Das Entstehen der Ausgleich- 


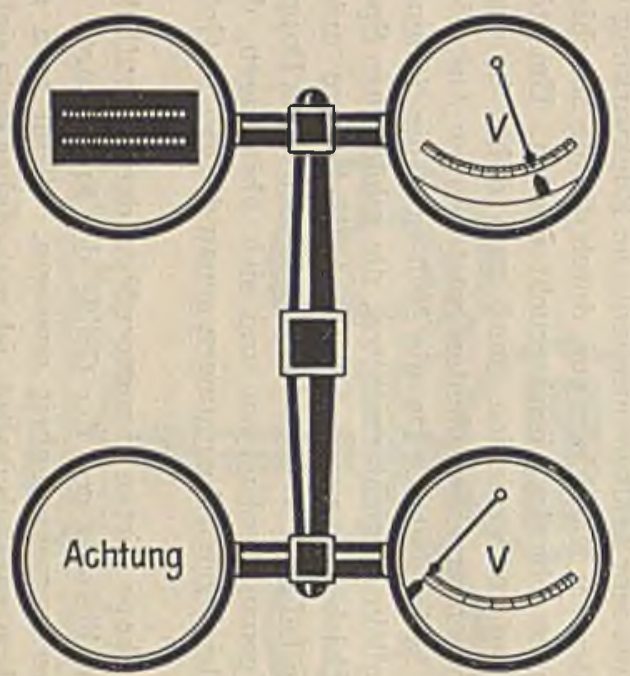

Bild 57. Meßeinrichtung mit Doppelfrequenzmesser, Doppelspannungsmesser, Nullspannungsmesser und einer an einen Umkehrtransformator angeschlossenen Phasenlampe.

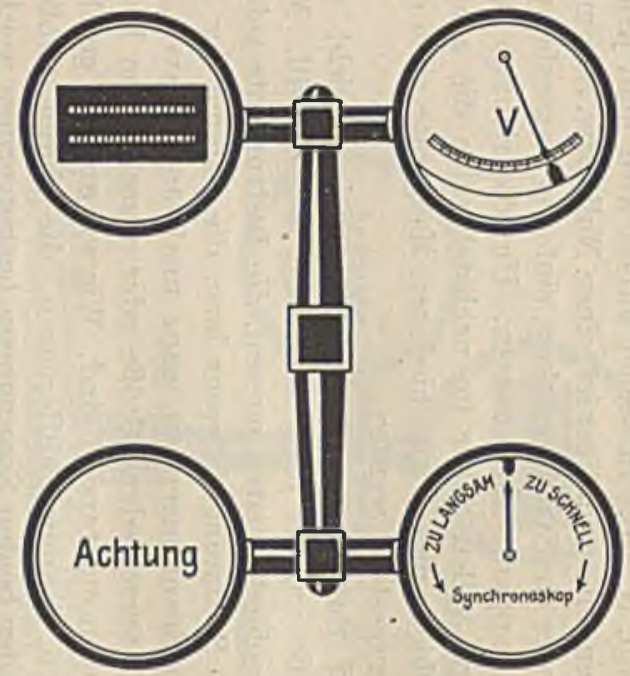

Bild 58. Meßeinrichtung mit Doppclfrequenzmesser, Doppelspannungsmesser, Synchronoskop und einer an einen Umkebrtransformator angeschlossenen Phasenlampe.

Tafel 18. Instrumentsätze für gemischte Schaltung. 
ströme an sich wird allerdings bei dem Parallelschalten mit dem Synchronoskop nicht verhindert, sondern es werden nur die schädlichen Ausgleichströme vermieden. Die wattlosen, durch verschiedene Größe der zu vergleichenden Spannungen entstehenden Ausgleichströme können jedoch durch einfaches Einstellen der Spannung nach den Angaben des Doppelspannungsmessers so klein gehalten werden, daß eine unnötige Beanspruchung der Schalterkontakte in jedem Falle vermieden wird. Die Synchronoskope werden daher namentlich bei schwierigeren Betrieben, bei denen sich absolute Spannungsgleichheit nur schwer erreichen läßt, ein sicheres und gefahrloses Parallelschalten ermöglichen. Sie bieten außerdem den Vorteil, daß durch den Drehsinn des Zeigers ohne weiteres der Sinn der erforderlichen Regelung der Drehzahl angegeben wird, und daß man es durch Beachtung des Drehsinns stets erreichen kann, daß die zugeschaltete Maschine sofort als Generator Last aufnimmt.

Die Phasenlampen bzw. die Lampenapparate werden bei allen Schaltungen nur als ergänzende Meßmittel zu anderen genaueren Synchronisiermeßgeräten benutzt. Durch sie wird die Betriebssicherheit der Parallelschalteinrichtung wesentlich erhöht, da sie durch ihr Aufleuchten bzw. Verlöschen dem Maschinisten anzeigen, da $B$ die Einrichtung ordnungsgemä $\beta$ arbeitet. In den meisten Fällen werden die einfachen Phasenlampen in Gehäusen, wie sie auf S. 27 beschrieben sind, benutzt. Der Lampenapparat wird nur noch in kleineren Kraftwerken, und auch da verhältnismäßig selten, angewendet. Er dient dann lediglich als Signalgeber für die Regelung der Drehzahl der zuzuschaltenden Maschine und bietet hierbei den Vorteil, daß er auf größere Entfernungen ablesbar ist. Er ersetzt daher in vielen Füllen eine besondere Befehlsübertragung zwischen Schaltbühne und Maschine.

\section{Vollständige Instrumontensätze.}

Die zur Parallelschalteinrichtung gehörigen Meßinstrumente werden zweckmäßig auf einem Wiandarm oder einer Säule zu einem einheitlichen Ganzen versinigt. Diese Anordnung bietet gegenüber dem Einbau der Instrumente in die Schalttafel den wesentlichen Vorzug, daß die Parallelschalteinrichtung als ein für alle Maschinensätze gemeinsam geltendes Meßgerät aus der Schaltwand hervortritt und daher von allen Seiten sichtbar ist. 
Auf den Tafeln 17 und 18 sind einige solcher Zusammenstellungen, wie sie von $\mathrm{S}$. \& $\mathrm{H}$. ausgeführt werden, schematisch dargestellt.

Bild 55 zeigt einen Instrumentsatz für Hellschaltung mit Doppelfrequenzmesser, Summenspannungsmesser und Phasenlampe. Der Summenspannungsmesser wird hierbei nach Schaltbild 8 an einen Umschalter angeschlossen, so $\mathrm{da} B$ er auch als Doppelspannungsmesser benutzt werden kamn. Die Phasenlampe ist so geschaltet, daß sie nur dann arbeitet, wenn das Instrument als Summenspannungsmesser geschaltet ist. Sie ist in ein Gehäuse mit Transparentscheibe eingebaut. Beim Aufleuchten der Phasenlampe erscheint das Signal „Achtung“ und macht den Maschinenwürter darauf aufmerksam, daß der Augenblick des Parallelschaltens nahe ist.

Bild 56 zeigt ebenfalls einen Instrumentsatz für Hellschaltung, jedoch ist hierbei an Stelle der einfachen Phasenlampe ein Lampenapparat benutzt, der durch den Drehsinn des Lichtscheins angibt, in welchem Sinne die zuzuschaltende Maschine geregelt werden muß. Diese Anordnung kommt jedoch, wie bereits im vorigen Abschnitt gesagt wurde, nur noch für kleinere Anlagen in Frage, bei denen eine besondere Befehlsübertragung zwischen Schaltbrett und Maschine gespart werden soll.

Bild 57 zeigt einen. Instrumentsatz für gemischte Schaltung mit Doppelfrequenzmesser, Doppelspannungsmesser, Nullspannungsmesser und Phasenlampe. Der Nullspannungsmesser liegt hierbei in der normalen Dunkelschaltung, während die an einen Umkehrtransformator angeschlossene Phasenlampe in Hellschaltung arbeitet (vgl. S. 37). Die Phasenlampe ist hierbei wieder in ein Gehäuse mit Transparentscheibe eingebaut und gibt durch ihr Aufleuchten das Achtungssignal zum Parallelschalten. Bei der reinen Dunkelschaltung ist der Instrumentsatz im wesentlichen der gleiche, jedoch liegt dann auch die Phasenlampe in Dunkelschaltung. Die Transparentscheibe des Lampengehäuses wird dann so ausgeführt, da $B$ anstelle des Achtungssignals ein Warnungssignal erscheint (vgl. S. 27).

Bild 58 zeigt ebenfalls eine, gemischte Schaltung, jedoch ist an Stelle des Nullspannungsmesscrs ein Synchronoskop verwendet, das durch den Drehsinn des umlaufenden Zeigers olne weiteres den Regelsinn für die zuzuschaltende Naschine angibt. Bei Phasengleichheit bleibt der Zeiger des Synchronoskops auf der 
oberen Kennmarke stehen. Die Phasenlampe dient hierbei wieder zur Kontrolle für das richtige Arbeiten des Synchronoskops und gibt das Achtungssignal zum Parallelschalten. Diese Anordnung ist als die vollkommenste Einuichtung für Kraftwerke mit schwierigen Betriebsverhältnissen anzusehen.

\section{m. Hilfs̊pparate.}

Die Verbindungen der Maschinen bzw. der Sammelschicnen mit den zur Parallelschaltung erforderlichen MeBinstrumenten werden bei den Siemens-Schuckertwerken dureh besondere Stöpselschalter hergestellt. Diese bieten gegenüber anderen Schaltern die Vorteile, daß sic einesteils bei Verwendung verschieden ausgebildeter Stecker ganz bestimmte Schaltungen zwangläufig festlegen, anderenteils aber die Möglichkeit falscher Schaltungen durch Verwendung einer beschränkten Anzahl Stecker ausschließen.
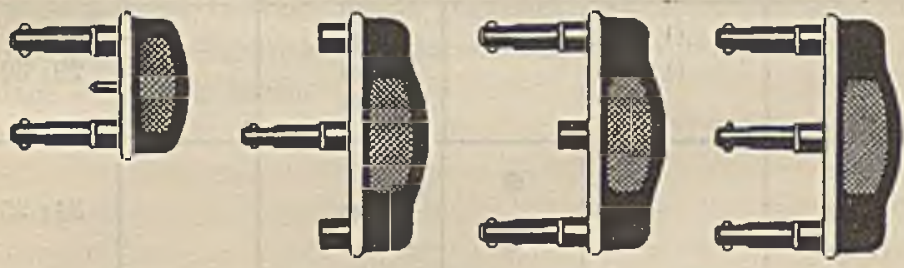

Bild 59 bis 62 .

Die Stöpselschalter bestehen aus einer in die Schalttafel eingebauten Steckeinrichtung und einem oder zwei für die ganze Anlage dienenden Steckern. Die Steckeinrichtungen selbst sind je nach der Schaltung zwei- oder dreipolig ausgeführt, während die Stecker einpolig, zweipolig und dreipolig hergestellt werden. Die zweipoligen Stecker werden außerdem mit großem und mit kleinem Abstande der Kontaktstifte ausgeführt. Um bei dreipoligen Steckeinrichtungen ein falsches Einstecken der zweipoligen kurzen Stecker zu vermeiden, erhalten sie einen besonderen, zwischen den beiden Kontakten angeordneten Sperrstift, der in eine an der entsprechenden Stelle der Steckeinrichtung angebrachte Öffnung eingreift. Die Einführungsöffnung für diesen Stift ist in den Schaltbildern durch einen kleinen Kreis dargestellt. 


\begin{tabular}{|c|c|c|c|c|c|}
\hline \multicolumn{2}{|c|}{$\begin{array}{c}\text { Steckvorrielitung } \\
\text { fUr }\end{array}$} & \multicolumn{3}{|c|}{$\begin{array}{l}\text { Btecker } \\
\text { fúr }\end{array}$} & \multirow{2}{*}{$\begin{array}{l}\text { Prssend } \\
\text { fur } \\
\text { Schaltung } \\
\text { Nr. }\end{array}$} \\
\hline jede Mnschine & Sammelschienen & $\begin{array}{l}\text { laufende } \\
\text { Maschine }\end{array}$ & $\begin{array}{l}\text { zuzuschaltende } \\
\text { Mrschine }\end{array}$ & $\begin{array}{l}\text { Sammel- } \\
\text { selienen }\end{array}$ & \\
\hline $\begin{array}{l}0 \\
0\end{array}$ & & & 0 & & $\begin{array}{c}1-4 ; 6-10 \\
13-16\end{array}$ \\
\hline $\begin{array}{l}\text { () } \\
\text { () }\end{array}$ & & & 0 & & $26--28$ \\
\hline $\begin{array}{l}0 \\
0 \\
0\end{array}$ & & & 0 & & 12 \\
\hline & & & 0 & & 23 \\
\hline $\begin{array}{l}0 \\
0 \\
0\end{array}$ & $\begin{array}{l}0 \\
0 \\
0\end{array}$ & & 0 & & $29 ; 30$ \\
\hline $\begin{array}{ll}0 & 0 \\
0 & 0 \\
0 & 0\end{array}$ & & 0 & 0 & & $24 ; 25$ \\
\hline $\begin{array}{ll}0 & 0 \\
0 & 0\end{array}$ & $\begin{array}{ll}\text { (1) } & 0 \\
\text { () } & 0 \\
\text { () } & 0\end{array}$ & & ? & 0 & $5 ; 11$ \\
\hline & & & $?$ & 0 & \\
\hline $\begin{array}{l}0 \\
0 \\
1) \\
0\end{array}$ & & $\dot{0}$ & 0 & & $18 ; 20-22$ \\
\hline $\begin{array}{ll}\text { () } & 0 \\
0 & 0 \\
0 & 0 \\
0 & 0\end{array}$ & & ? & 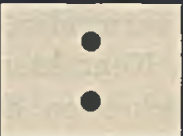 & & 19 \\
\hline
\end{tabular}

Tafel 19. Steckrorrichtungen und Stecker. 
Um einen leichten Ubberblick über dic erforderlichen Stöpsclschalter zu bekommen, sind in der Tabelle auf S. 54 die für die verschiedenen Schaltungen nötigen Steckvorrichtungen und Stecker zusammengestellt.

Die Hauptschalter, durch die die Maschinen beim Parallel. schalten an die Sammelschienen angeschlossen werden, werden meistens als Olschaltor mit Schutzwiderstand ausgeführt. Diese sog. Schutzschalter haben neben den Hauptkontaktfedern besondere isolierte Vorkontakte, an die ein Schutzwiderstand angeschlossen ist. Beim Finlegen des Kontaktmessers wird hierbei der Stromschluß zunächst über den Schutzwiderstand hergestellt. Bei der Weiterbewegung des Kontaltmessers in seine Betriebsstellung wird der Schutzwiderstand kurzgeschlossen. Die Schutzschalter bieten beim Parallelschalten den wesentlichen Vorteil, daß die bei schlechtem Parallelschalten auftretenden Stromstöße und Spannungswellen so gemildert werden, $\mathrm{d} a ß$ eine unzulässige Beanspruchung der Maschinen vermieden wird. Auf diese Weise ist eine gewisse Gewähr dafür gegeben, daß auch bei schlechter Bedienung der Parallelschalteinrichtung keine Beschädigung der Anlage eintreten kann. 


\section{Vollständige Schaltungen.}

\section{Allgemeines über die Auswahl ciner passenden Schaltung.}

Bei der Ausführung der Parallelschalteinrichtungen hat man zwischen drei Hïglichkeiten zu unterscheiden. Entweder führt man die Phasenvergleichung zwischen Generator und Sammelschienen, oder zwischen Generator und Generator, oder endlich an den Schaltkontakten der Hauptschalter aus.

Die einfachsten Schaltungen ergeben sich bei der Phasenvergleichung zwischen Generator und Sammelschienen. Man wird diese Art der Schaltung stets dann mit Erfolg benutzen, wenn man Anlagen mit nicht allzu hoher Spannung zu schalten hat. Für Spannungen bis 250 Volt werden die Schaltungen direkt oder besser halbindirekt ausgeführt, während man für höhere Spannungen die indirekte Schaltung mit Spannungswandlern benutzt. Um bei Mehrfachsammelschienen ein Schalten auf falsche Sammelschienen zu vermeiden, sind bei diesen Schaltungen besondere Hilfskontakte an den Trennschaltern erforderlich, die die zugehörige Parallelschalteinrichtung zwangläufig ein- und ausschalten. Gleichzeitig werden durch diese Hilfskontakte auch Signallampen eingeschaltet, die ohne weiteres anzeigen, welcher Trennschalter geschlossen ist. Zum Parallelschalten der verschiedenen Sammelschienensysteme sind hierbei keine besonderen Einrichtungen erforderlich, da man dic Parallelschaltung durch unmittelbares Vergleichen der an die rerschiedenen Sammelschienen angeschlossenen Generatoren ausführen kann.

Liegen zwischen den Generatoren und den Sammelschienen Transformatoren, die die Generatorspannung auf eine wesentlich höhere Sammelschienenspannung hinauftransformieren, so wird die Phasenvergleichung zwischen Generator und Sammelschicnen ungünstig, da die erforderlichen Spannungswandler für die hohe Sammelschienenspannung zu teuer werden und erheblichen Raum beanspruchen. Man führt daher in diesem Falle die Phasenvergleichung zwischen Gencrator und Generator aus. Hierbei hat man gegenüber der vorher beschriebenen Schaltwcise den Vorteil, daß alle Spannungswandler nur für die verbältnismäßig niedrige Generatorspannung zu bemessen sind und daher klein und billig werden. Allerdings ist hierbei Voraussetzung, da $\beta$ 
die Innenschaltung der zu den einzelnen Generatoren gehörigen Hochspannungstransformatoren bei allen Transformatoren gleichartig ausgeführt ist, so daß bei Phasengleichheit auf der Unterspannungsseite auch die entsprechenden Oberspannungsseiten phasengleich sind. Sollen etwaige von anderen Kraftwerken kommende Speiseleitungen unmittelbar zu den auf der Oberspannungsseite liegenden Sammelschienen parallel geschaltet werden, so kann dies nur durch Phasenvergleichung der Oberspannungsseite der Speiseleitungen mit der Unterspannungsseite des Kraftwerkes erfolgen. Da die wohl unter sich phasengleichen Generatoren infolge der Zwischenschaltung der Hochspannungstransformatoren aber nicht mit den Sammelschienen phasengleich zu sein brauchen, müssen die zum Synchronisieren der Speiseleitungen benutzten Spannungswandler eine etwaige zwischen der Unter- und Oberspannungsseite bestehende, durch die Schaltung der Transiormatoren bedingte Phasenverschiebung berücksichtigen (vg]. S. 106). Sie müssen so geschaltet sein, daß ihre Sekundärspamung bei Synchronismus auf der Oberspannungsseite mit der Generatorspannung phasengleich ist. Hierauf ist bei der erstmaligen Inbetriebsetzung derartiger Anlagen besonders zu achten. Für die normale Betriebsführung werden jedoch hierdurch keinerlei Schwierigkeiten verursacht. Bei Anlagen mit Mehrfachsammelschienen werden in der glcichen Weise wie bei der Phasenvergleichung zwischen Generator und Sammelschienen besondere Hilfskontakte an den Trennschaltern benutzt, die die zugehörige Parallelschalteinrichtung nebst Signallampe zwangläufig ein- und ausschalten. Zum Parallelschalten der verschiedenen Sammelschienensysteme sind auch hierbei keine besonderen Einrichtungen erforderlich, da man die Parallelschaltung wieder durch unmittelbares Vergleichen der an die verschiedenen Sammelschienen angeschlossenen Generatoren ausführen kann.

Die Phasenvergleichung an den Schalterkontakten ist besonders für Anlagen mit Mehrfachsammelschienen vorteilhaft. Sic vermeidet alle Unsicherheiten, die durch die vielfachen Schaltmöglichkeiten entstehen, dadurch, daß die Phasenvergleichung unmittelbar an dem die Parallelschaltung vollziehenden Maschinenschalter, also vor den Abzweigungen nach den einzelnen Sammelschienensystemen, erfolgt. Sind die Spannungen an diesem Schalter phasengleich, so kann die Parallelschaltung ganz, un- 
abhängig von der jeweiligen Schaltung der Sammelschienen erfolgen. Zum Parallelschalten der verschiedenen Sammelschienensysteme ist hierbei eine besondere Einrichtung erforderlich, die in gleicher Weise wie die Parallelschalteinrichtungen der einzelnen Maschinen ausgefübrt wird. Dem Vorteil der großen Einfachheit dieser Schaltung steht der Nachteil gegenüber, daß für jede Maschine zwei Satz Spannungswandler erforderlich sind. Dieser Nachteil ist jedoch für mittlere Spannungen nicht erheblich, dn hierbei die Kosten der Spannungswandler gegenüber den Kosten der ganzen Anlage nicht mehr ins Gewicht fallen. Bei Anlagen mit hoher Spannung, bei denen immer ein Generator zusammen mit einem Hochspannungstransformator eine Schalteinheit bildet und bei denen demgemäß sämtliche Ausschalter hinter den Transformatoren auf der Oberspannungsseite liegen, sind indessen die Kosten und der Raumbedarf der Spannungswandler sehr crheblich, da dann sämtliche Spannungswandler für die hohe Sammelschienenspannung ausgeführt werden müssen. Man wird daher in diesem Falle die Phasenvergleichung zwischen Generator und Generator vorziehen.

\section{Phasenvergleichung zwischen Generator und Sammelsehienen.}

a. Dunkelschaltung mit Nullspanmungsmesser.

Schaltljild 1 zeigt die direkte Schaltung mit Nullspannungsmesser und Phasenlampen in Dunkelschaltung. Die Schaltung ist für Spannungen bis 250 Volt anwendbar. Der Instrumentsatz bestcht aus einem Doppelfrequenzmesser, einem Doppelspannungsmesser, einem Nullspannungsmesser und zwei Phasenlampen $P_{1}$ und $P_{2}$. Die Verteilung der beim Parallelschalten auftretenden doppelten Spannung auf zwei Phasenlampen ist bei der direkten Schaltung stets erforderlich, da die Maschinen durch die Hauptschalter stets allpolig von den Sammelschienen abgetrennt werden und eine unmittelbare Verbindung der Hauptsammelschienen mit den Hilfssammelschienen wegen der Vergrößerung der Kurzschlußgefahr in keinem Falle zulässig ist. Die Phasenlampen und der Nullspannungsmesser sind daher für die einfache Netzspannung zu bemessen. Um eine gleiche Verteilung der Spannung auf die beiden in Reihe geschalteten Phasenlampen zu erreichen, ist 
parallel zur Phasenlampe $P_{2}$ ein Ersatzwiderstand $R$ eingeschaltet, der den gleichen Wattverbrauch wie der Nullspannungsmesser aufweist. Bei Verwendung der früheren, überlastbaren Nullspannungsmesser konnte man unter Umständen auf diesen Ersatzwiderstand $R$ verzichten, da der Eigenverbrauch dieser Instrumente gegenüber dem der Phasenlampe nicht in Betracht laam. Bei dem newen, auf S. 35 beschriebenen Nullspannungsmesser mit Vorschaltlampe ist dagegen der Ersatzwiderstand in jedem Falle erforderlich, da der Eigenverbrauch dieses Nullspannungsmessers ebenso groß ist, wie der der parallel zu ihm liegenden Phasenlampe. Bei der Bemessung der Größe dieses Ersatzwiderstandes muß man außer dem Wattverbrauch auch noch die Widerstandsänderung des Nullspannungsmessers berücksichtigen. Dies geschieht in einfachster Weise dadurch, daß man als Ersatzwiderstand $R$ eine gleiche Glühlampe wie die Vorschaltlampe des Nullspannungsmessers verwendet. Zur Betätigung der Schalteinrichtung ist für die ganze Anlage nur ein zweipoliger Stecker erforderlich, der in den Steckkontakt der zuzuschaltenden Maschine eingeführt wird.

Schaltbild 2 zeigt die halbindirekte Schaltung mit Nullspannungsmesser und Phasenlampe in Dunkelschaltung. Die Schaltung ist, ebenso wie Schaltung 1, für Spannungen bis 250 Volt bestimmt. Sic unterscheidet sich von Schaltung 1 dadurch, daß zwischen die Sammelschienen und die Meßeinrichtung ein im Verhältnis 1:1 übersetzender Isoliertransformator $J T$ eingeschaltet ist. Durch die Zwischenschaltung des Isoliertransformators werden die Sammelschienen elektrisch vollkommen von der Meßschaltung getrennt und es wird ermöglicht, die Meßschaltung in gleicher Weise wie die indirekte Schaltung mit einer einpoligen Verbindung auszuführen (vgl. S. 8). Die unsichere Verteilung der Spannung auf zwei Phasenlampen wird somit hierbei vermieden. Am Nullspannungsmesser und an der Phasenlampe tritt dam die doppelte Netzspannung auf. Die Sekundärwickelung des Isoliertransformators darf nicht geerdet werden, da man durch die Erdung einen Pol der Anlage an Erde legen würde. Zur Betätigung der Schalteinrichtung ist ebenso wie bei Schaltbild 1 nur ein zweipoliger Stecker erforderlich, der in den Steckkontakt der zuzuschaltenden Maschine eingeführt wird.

Schaltbild 3 zeigt eine indirekte Schaltung für Spannungen 
bis 250 Volt, mit Nullspannungsmesser und Phasenlampe in Dunkelschaltung. Die Schaltung unterscheidet sich von der vorher beschriebenen dadurch, daß auch an den Hilfssammelschienen ein Spannungswandler angeschlossen ist und daß beide Spannungswandler auf cine Sekundärspamnung von 110 Volt übersetzon. Der Nullspannungsmesser und die Phasenlampe sind daher in diesem Falle nur für die doppelte Sekundärspannung der Spannungswandler, also für $2 \times 110$ Volt zu bemessen. Da die Steckvorrichtungen bei dieser Schaltung an der vollen Maschinenspannung liegen, ist der Anwendungsbereich dieser Schaltung auf Niederspannungsanlagen beschränkt. Zur Bedienung der Parallelschalteinrichtung ist ebenso wio bei den Schaltbildem 1 und 2 nur ein zweipoliger Stecker erforderlich, der in den Steckkontalkt der zuzuschaltenden Maschine eingeführt wird.

Schaltbild 4 zeigt eine indirekte Schaltung für Hochspannung, ebenfalls mit Nullspannungsmesser und Phasenlampe in Dunlkelschaltung. Der Instrumentsatz ist wieder der gleiche wie bei Schaltbild 3. Der Nullspannungsmesser und die Phasenlampe sind wieder für die doppelte Sekundärspannung der Spannungswandler, also für $2 \times 110$ Volt zu bemessen. Um das Bedienen der Schaltung gefahrlos zu machen, sind die Steckvorrichtungen auf die Niederspannungsseite verlegt. Es ist daher für jede Maschine ein besonderer Maschinenspannungswandler vorgesehen. Um zu verhüten, daß abgeschaltete Maschinen bei versehentlichem falschen Stöpseln durch Rücktransformicrung des:zugehörigen Spannungswandlers unter Spannung gesetzt werden, sind bei dieser Schaltung an den Trennschaltern der Maschinen besondere Hilfskontakte angebracht, die die Parallelschalteinrichtung bei ausgeschalteten Trennschaltern unterbrechen.

Schaltbild 5 zeigt die indirekte Hochspannungsschaltung mit Nullspannungsmesser bei Mehrfachsammelschienen. Durch die auf der rechten Seite des Schaltbildes angegebene Steckeinrichtung können die beiden Sammelschienensysteme wahlweise auf die Parallelschalteinrichtung geschaltet werden. Damit man stets ohne weiteres erkennen kann, welches der beiden Sammelschienensysteme eingeschaltet ist, sind hierbei farbige Signallampen angebracht, die beim Einführen des Steckers aufleuchten. An den Steckvorrichtungen der. Maschinen sind entsprechende Signallampen angebracht, die aber unabhängig vom Stecker durch die 
Hilfskontakte an den Trennschaltern eingeschaltet werden. Man kann daher an der Farbe der brennenden Signallampen stets von vornherein erkennen, welche Trennschalter eingelegt und welche Sammelschienen an dic Meßeinrichtung angeschlossen sind. Ein versehentliches Schalten auf falsche Sammelschienen ist somit kaum noch möglich. Gleichzeitig mit der Signalgebung erfüllen die Hilfskontakte noch den gleichen Zweck wie bei Schaltbild 4 . Zur Bedienung der ganzen Anlage ist ein kurzer und ein langer zweipoliger Stecker erforderlich. Soll eine Maschine neu in Betrieb genommen werden, sind dic Stecker so zu stecken, daß an der Maschinen- und an der Sammelschienen-Steckeinichtung die gleichfarbigen Lampen leuchten. Sollen dagegen die verschiedenen Sammelschienensysteme parallel geschaltet werden, so vergleicht man eine an dem einen Sammelschienensystem bereits laufende Maschine mit dem anderen Sammelschienensystem. In diesem Falle müssen daher verschiedenfarbige Lampen brennen. Etwaige von einem fremden Kraftwerk kommende Speiseleitungen werden in gleicher Weise geschaltet wie die einzelnen Maschinen.

\section{b. Hellschaltung mit Summenspanuungsmesser.}

Schaltbild 6 zeigt die direkte Schaltung mit Summenspannungsmesser und Phasenlampen in Hellschaltung für Anlagen bis 250 Volt Netzspannung. Der Instrumentsatz besteht aus einem Doppelfrequenzmesser, einem als Summenspannungsmesser dienenden Doppelspannungsmesser (vgl. S. 36), zwei Phasenlampen $P_{1}$ und $P_{2}$, sowie einem Ersatzwiderstand $R$. Die Verteilung der Summenspannung auf zwei Phasenlampen ist hier aus demselben Grunde wie bei Schaltung 1 erforderlich. Sie ist jedoch bei der Hellschaltung deshalb besonders unangenehm, da hierbei die Meßgenauigkeit des Summenspannungsmessers unmittelbar von der Verteilung der Spannung beeinflußt wird. Der Ersatzwiderstand $R$ muß daher genau den gleichen Widerstand wie das als Summenspannungsmesser benutztc Meßwerk des Doppelspannungsmessers besitzen. Der Summenspannungsmesser und die Phasenlampen sind für die einfache Netzspannung zu bemessen. Zur Betätigung der Schalteinrichtung ist für die ganze Anlage wieder nur ein zweipoliger Stecker erforderlich, der in den Steckkontakt der zuzuschaltenden Naschine eingeführt wird.

Schaltbild 7 zeigt die halbindirekte Schaltung mit Summen- 
spannungsmesser, ebenfalls für Anlagen bis 250 Volt Netzspannung. Durch die Zwischenschaltung des Isoliertransformators werden ebenso wic bei Schaltbild 2 die Sammelschienen elektrisch vollkommen von der Meßschaltung getrennt, so daß bei Vermeidung jeder Kurzschlußgefahr die denkbar einfachste Schaltung erreicht wird. Man kann hierbei ohne weiteres die Hilfssammelschienen cinpolig mit der transformierten Netzspannung verbinden und erzielt auf diese Weise eine größere Meßgenauigkeit. Da der Summenspannungsmesser die ungeteilte Spannung anzeigt, kommt man mit nur einer parallel zum Summenspannungsmesser angeschlossenen Phasenlampe aus. Phasenlampe und Summenspannungsmesser sind hierbei für die doppelte Netzspannung zu bemessen. Um einen normalen Doppelspannungsmesser als Summenspannungsmesser zu benutzen, muß man vor das an die Summenspannung angelegte Meßwerk einen Ergänzungswiderstand $R$ schalten, der den gleichen Widerstandswert wie das Meßwerk besitzt. Dic Schalteinrichtung wird ebenso wie die vorhergehende nur mit einem zweipoligen Stecker betätigt, der in den Steckkontakt der zuzuschaltenden Maschine eingeführt wird.

Schaltbild 8 ist lediglich cine vollkommenere Ausführung der in Schaltbild 7 angegebenen halbindirekten Schaltung. Während man beim Parallelschalten nach Schaltbild 7 die Spannung des zuzuschaltenden Generators durch den nur kurzzeitig auftretenden Höchstausschlag des Summenspannungsmessers mißt, ist bei Schaltbild 8 ein besonderer Umschalter vorgesehen, durch den der Summenspannungsmesser zeitweilig als Doppelspannungsmesser geschaltet wird. Steht der doppelpolige Umschalter in der Schaltstellung $S$, so ist der Summenspannungsmesser über den Vorwiderstand $R$ an die Summenspannung angeschlossen. Gleichzeitig ist die Phasenlampe eingeschaltet, so daß beim Höchstwert des Ausschlages die Phasenlampe voll brennt und somit das Achtungssignal zum Einschalten gibt. Ist dagegen der Umschalter auf dic Stellung $\boldsymbol{M}$ geschaltet, so liegt der Summenspannungsmesser ohne Vorwiderstand an der Spannung der zuzuschaltenden Maschine. Da der Zeigerausschlag jetzt dauernd bestehen bleibt, kann die Spannung der zuzuschaltenden Maschine sicherer eingestellt werden. Eine Verwechselung der beiden Schaltstellungen, die zu einem falschen Schalten führen könnte, 
ist hierbei nicht möglich, da im letztgenannten Falle die Phasen lampe ausgeschaltet ist.

Schaltbild 9 zeigte eine indirckte Schaltung für Spannungen bis 250 Volt mit Summenspannungsmesser und Phasenlampe in Hellschaltung. Die Schaltung unterscheidet sich von der vorher beschriebenen dadurch, daß auch an den Hilfssammelschicnen ein Spamnungswandler angeschlossen ist und daß beide Spammungswandler auf eine Sekundürspannung von 110 Volt übcrsetzen. Der Summenspannungsmesser und die Phasenlampe sind daher in diesem Falle nur für die doppelte Sekundärspannung der Spannungswandler, also für $2 \times 110$ Volt zu bemessen. Da dic Steckvorrichtungen hierbei an der vollen Maschinenspannung liegen, ist der Anwendungsbereich dieser Schaltung, ebenso wie bei Schaltbild 3, auf Nicderspannungsanlagen beschränkt. Zur Bedienung der Parallelschaltcinrichtung ist nur ein zweipoliger Stecker erforderlich, der in den Steckkontakt der zuzuschaltenden, Maschine eingeführt wird.

Schaltbild 10 zeigt die indirekte Schaltung für Hochspannung, ebenfalls mit Summenspannungsmesser und Phasenlampe in Hellschaltung. Der Instrumentsatz ist der gleiche wie bei Schaltbild 9. Der Summenspannungsmesser und die Phasenlampe sind wieder für die doppelte Selzundärspannung der Spannungswandler, also für $2 \times 110$ Volt zu bemessen. Um das Bedienen der Schaltung gefahrlos zu machen, sind die Steckvorrichtungen auf die Niederspannungsseite verlegt. Es ist daher für jede Maschine ein besonderer Maschinen-Spannungswandler vorgesehen. Die Trennschalter der Maschinen sind in gleicher Weise wie bei Schaltung 4 mit Hilfskontakten zur Vermeidung einer gefährlichen Rücktransformierung versehen. Zur Betätigung der Schalteinrichtung ist ein zweipoliger Stecker erforderlich, der in den Steckkontakt der zuzuschaltenden Maschine eingeführt wird.

Schaltbild 11 zeigt endlich die indirekte Hochspannungsschaltung mit Summenspannungsmesser bei Mehrfachsammelschienen. Der Instrumentsatz ist der gleiche wie bei Schaltbild 10. Die Anordnung der Schaltung ist bis auf die für die Hellschaltung erforderliche Uberkreuzung der Sekundärleitungen der Sammelschienentransformatoren dieselbe, wie bei Schaltbild 5. Zur Bedienung der ganzen Anlage ist ein kurzer und ein langer zweipoliger Stecker erforderlich. 


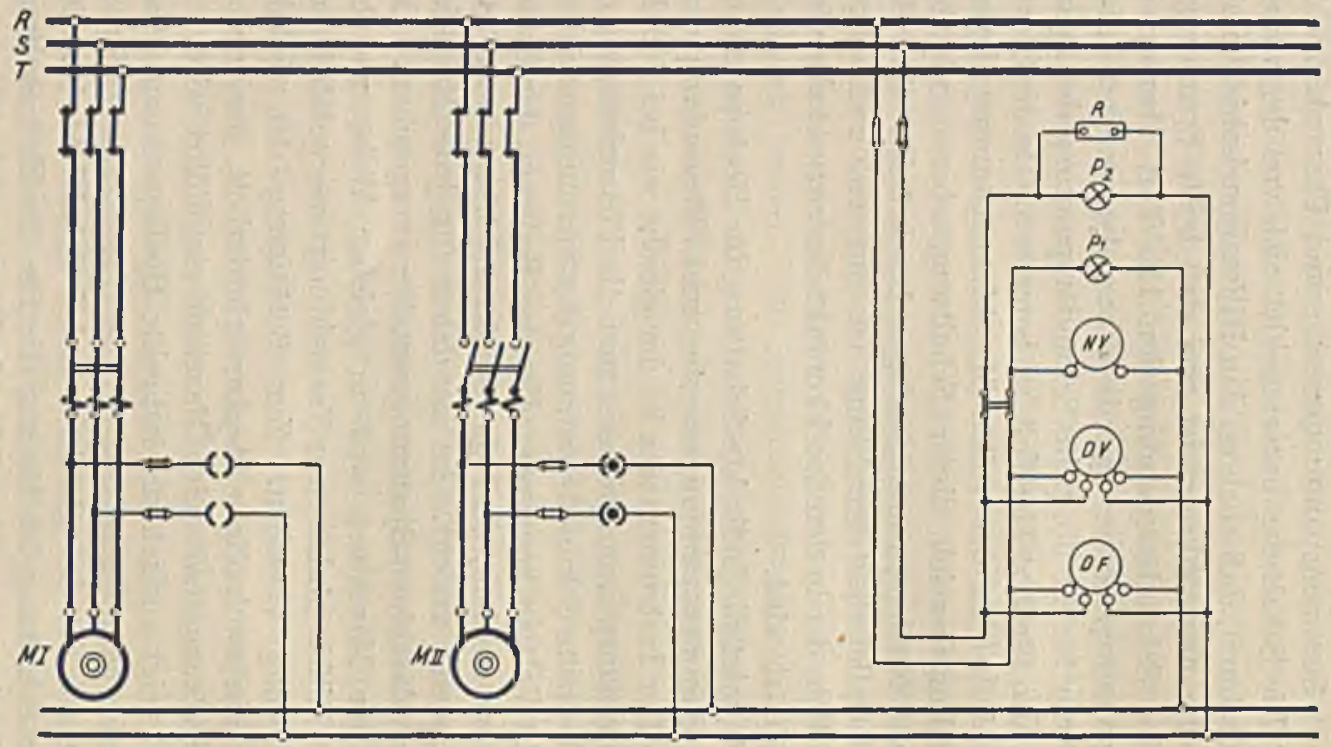

Für die Bedienung der Schaltung ist nur 1 kurzer zweipoliger Stecker erforderlich, der in die Steckeinrichtung der zuzuschaltenden Maschine eingeführt wird.

Schaltbild 1. Phasentergleichung zwischen Generator und Sammelschienen. Direkte Schaltung mit Nullspannungsmesser und Phasenlampen in Dunkelschaltung. (Bild 63.) 


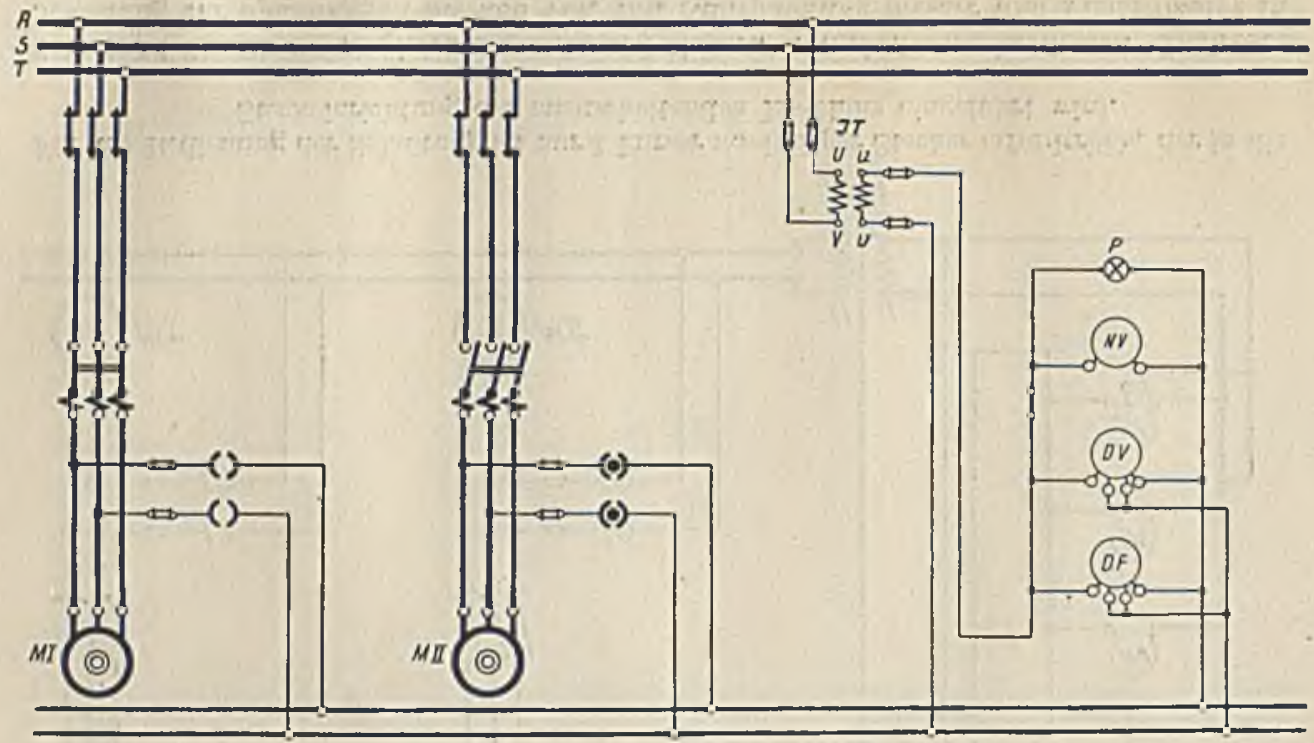

Für die Bedienung der Schaltung ist nur 1 kurzer zweipoliger Stecker erforderlich, der in die Steckeinrichtung der zuzuschaltenden Maschine eingeführt wird.

Schaltbild 2. Phasenvergleichung zwischen Generator und Sammelschienen. IIalbindirekte Schaltung mit Nullspannungsmesser u. Phasenlampe in Dunkelschaltung. (Bild 64.) 




Für die Bedienung der Schaltung ist nur 1 kurzer zweipoliger Stecker erforderlich, der in die Steckeinrichtung der zuzuschaltenden Maschino eingeführt wird.

Schaltbiłd 3. Phasenvergleichung zwischen Generator und Sammelschienen. Indirekte Schaltung für Spannungen bis 250 Volt mit Nullspannungsmesser und Phasenlampe in Dunkelschaltung. (Bild 65.) 


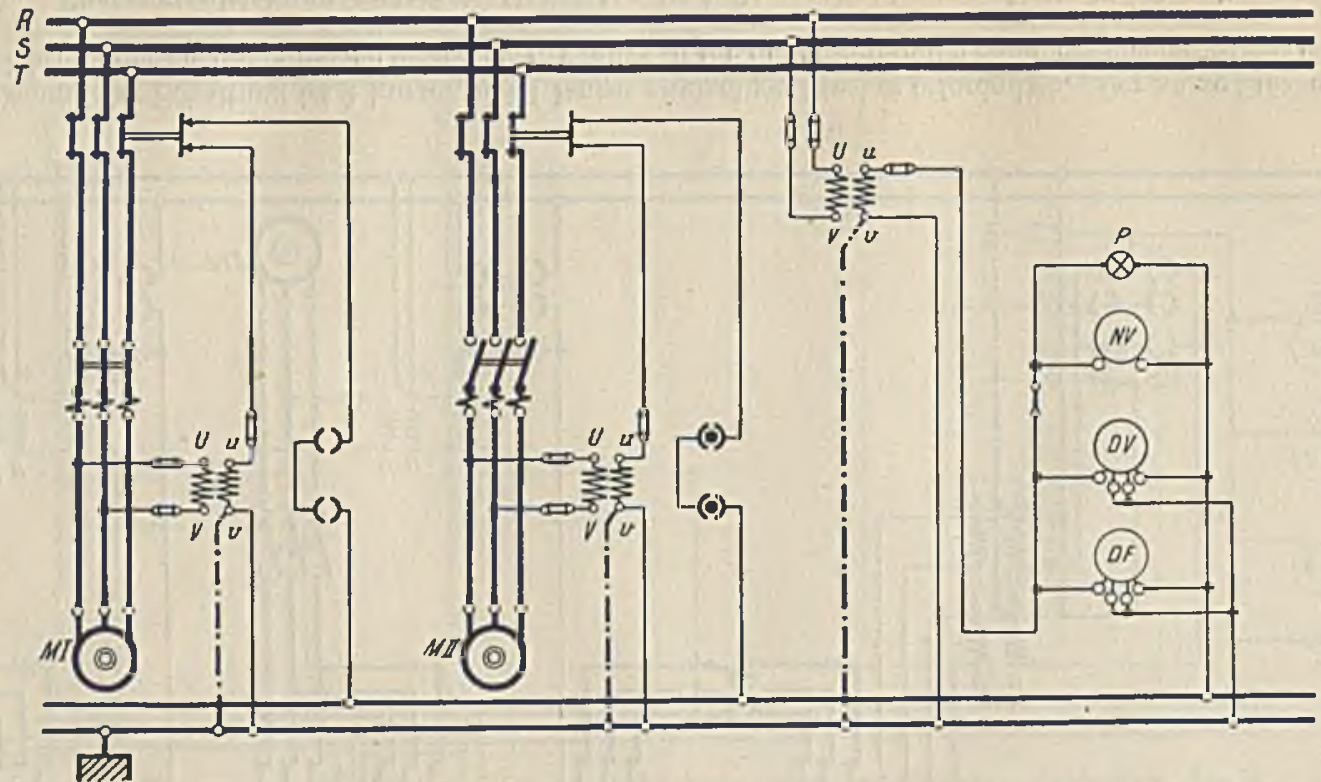

Für die Bedienung der Schaltung ist nur 1 kurzer zweipoliger Stecker erforderlich, der in dio Steckeinrichtung der zuzuschaltenden Maschine eingeführt wird.

Schaltbild 4. Phasenvergleichung zwisehen Generator und Sammelschienen. Indirekte Sehaltung mit Nullspannungsmesser und Phasenlampe in Dunkelschaltung. (Bild 66.) 


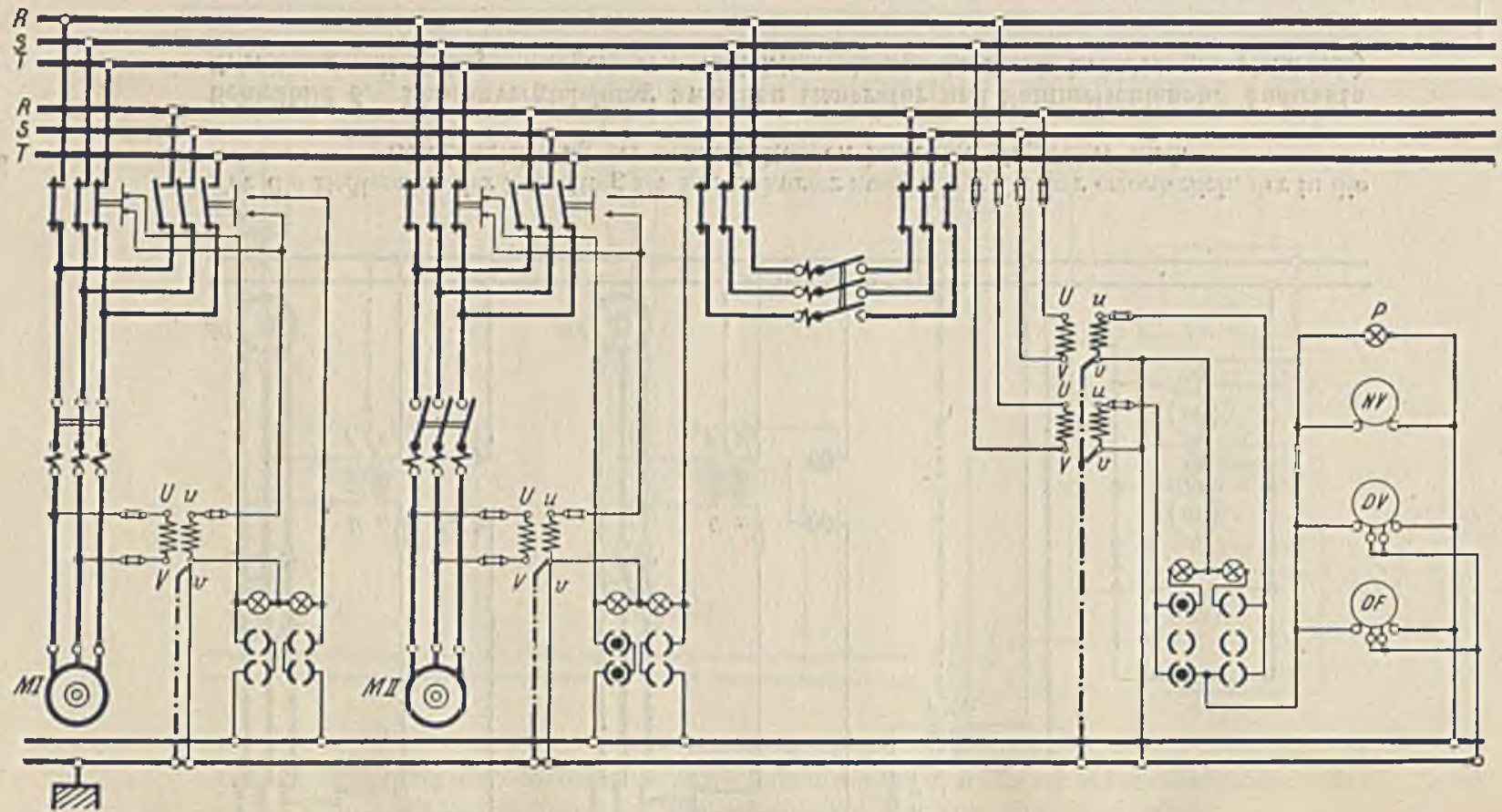

Für die Bedienung der Schaltung ist 1 kurzer und 1 langer zweipoliger Stocker erforderlich. Der kurze Stecker wird in dio Steckeinrichtung der zuzuschaltenden Maschine, der lange in die des gowünschten Sammelschienensystems eingeführt.

Schnltbild 5. Phasenvergleichung zwischen Generntor und Sammelschienen. Indirekte Schaltung mit Nullspannungsmesser und Phasenlampo in Dunkelschaltung; für Doppelsammelschienen. (Bild 67.) 


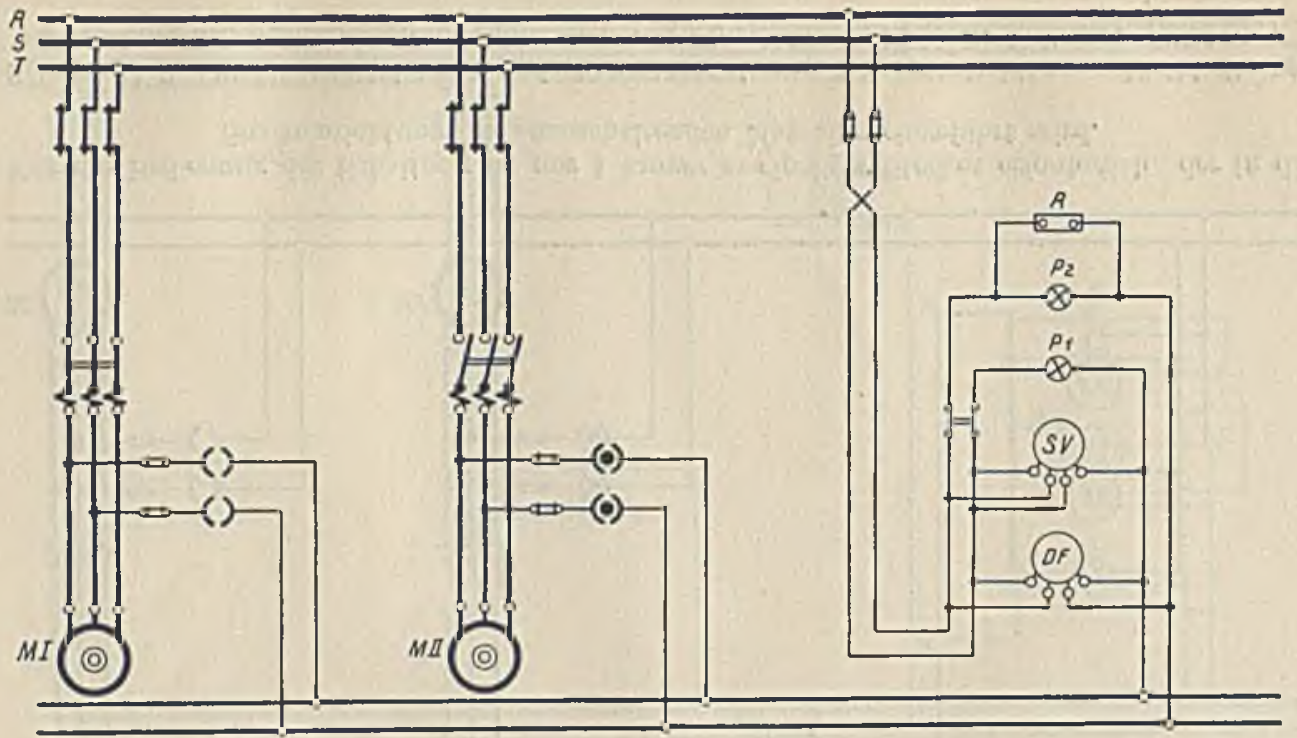

Für dio Bedienung der Schaltung ist nur 1 kurzer zweipoliger Stecker erforderlich, der in die Steckeinrichtung der zuzuschaltendon Maschine eingeführt wird.

Schaltbild 6. Phasonverglcichung zwischen Generator und Sammelsehionon. Direkte Sehaltung mit Summenspannungsmesser und Phasenlampen in Hellsehaltung. (Bild 68.) 


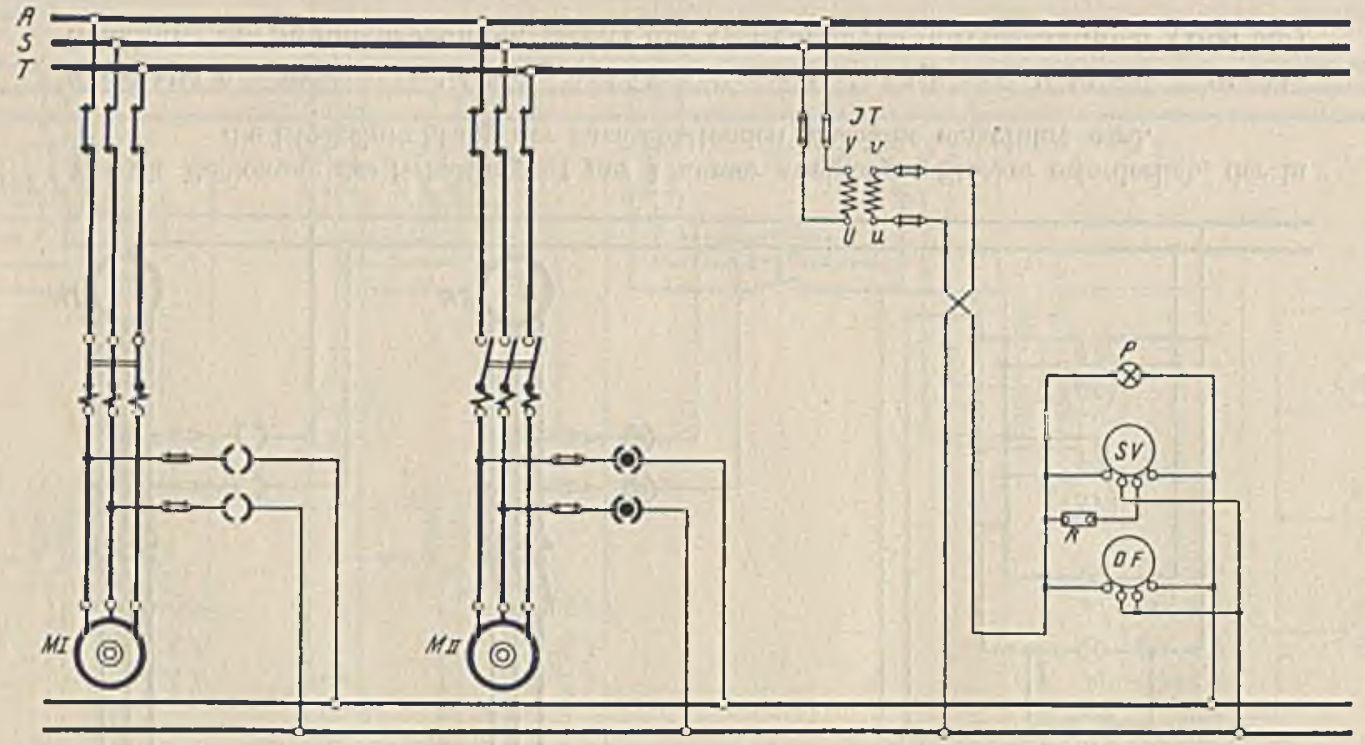

Für die Bedienung der Sehaltung ist nur 1 kurzer zweipoliger Stecker erforderlich, der in die Steckoinrichtung der zuzuschaltenden Maschino eingeführt wird.

Schaltbild 7. Phasenvergleichung zwischen Generator and Sammelschienen. Halbindirekte Schaltung mit Summenspannungsmesser und Phasenlampe in Hellschaltung. (Bild 69.) 


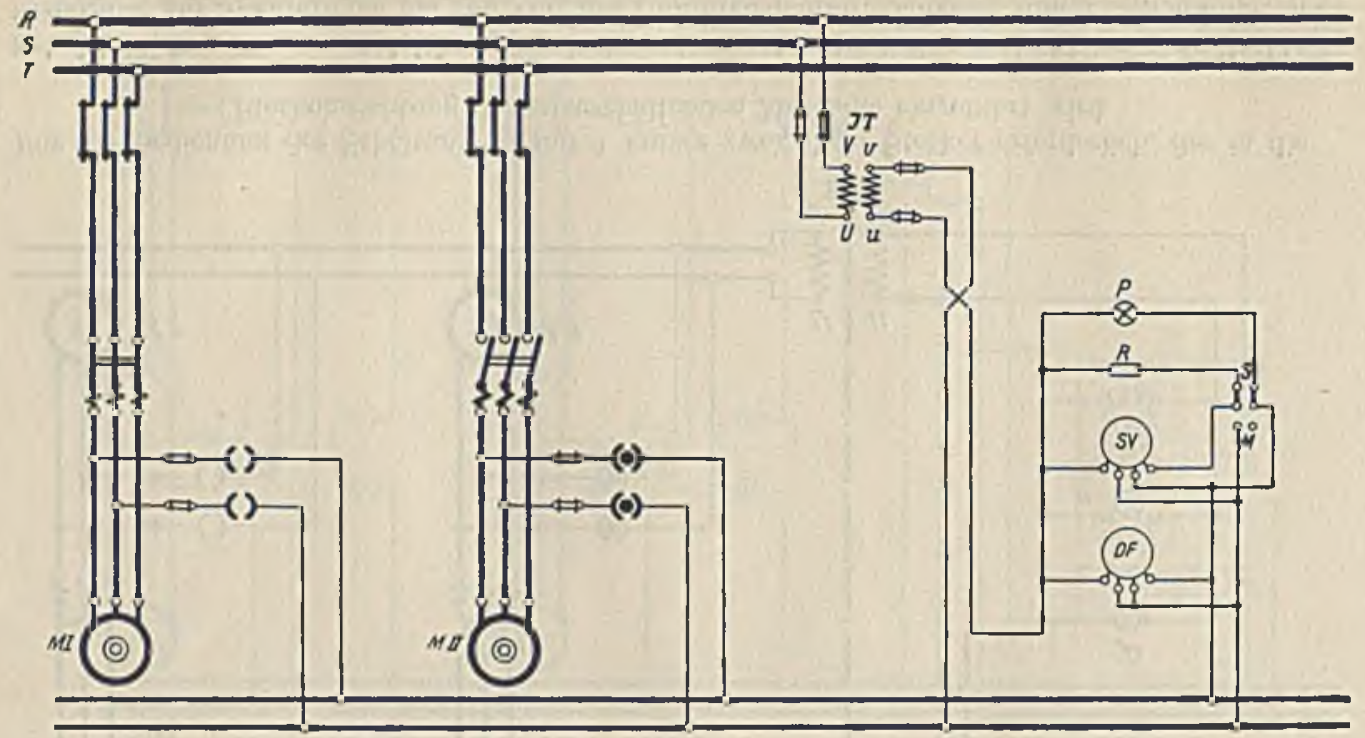

Fuir die Bedionung der Schaltung ist nur 1 kurzer zweipoliger Stecker erforderlich, der in die Steckeinrichtung der zuzuschaltenden Maschine eingeführt wird.

Schaltbild 8. Phasenvergleichung zwischen Generntor und Sammelsehienen. Halbindirckte Schaltung mit umschaltbarem Summenspannungsmesser und Phasenlampe in Hellschaltung. (Bild 70.) 


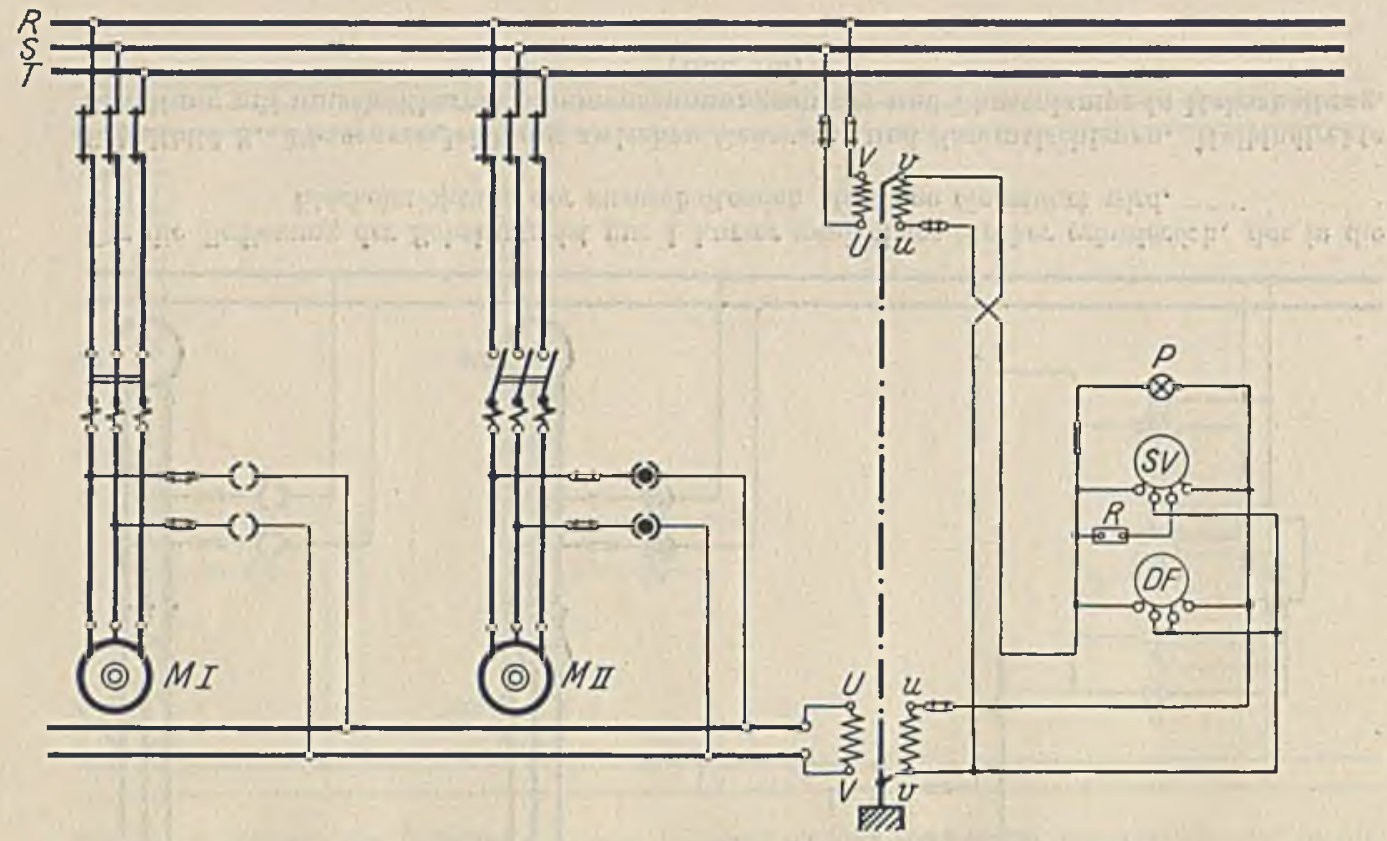

Für die Bedienung der Schaltung ist nur 1 kurzer zweipoliger Stecker erforderlich, der in dio Steckeinrichtung der zuzuschaltenden Maschine eingeführt wird.

Schaltbild 9. Phasenvergleichung wwisehen Generator und Sammelschienen. Indirokto Sclinltung für Spannungen bis 250 Volt mit Summenspannungsmesser und Phasenlampe in Hellsehaltung. (Bild 71.) 


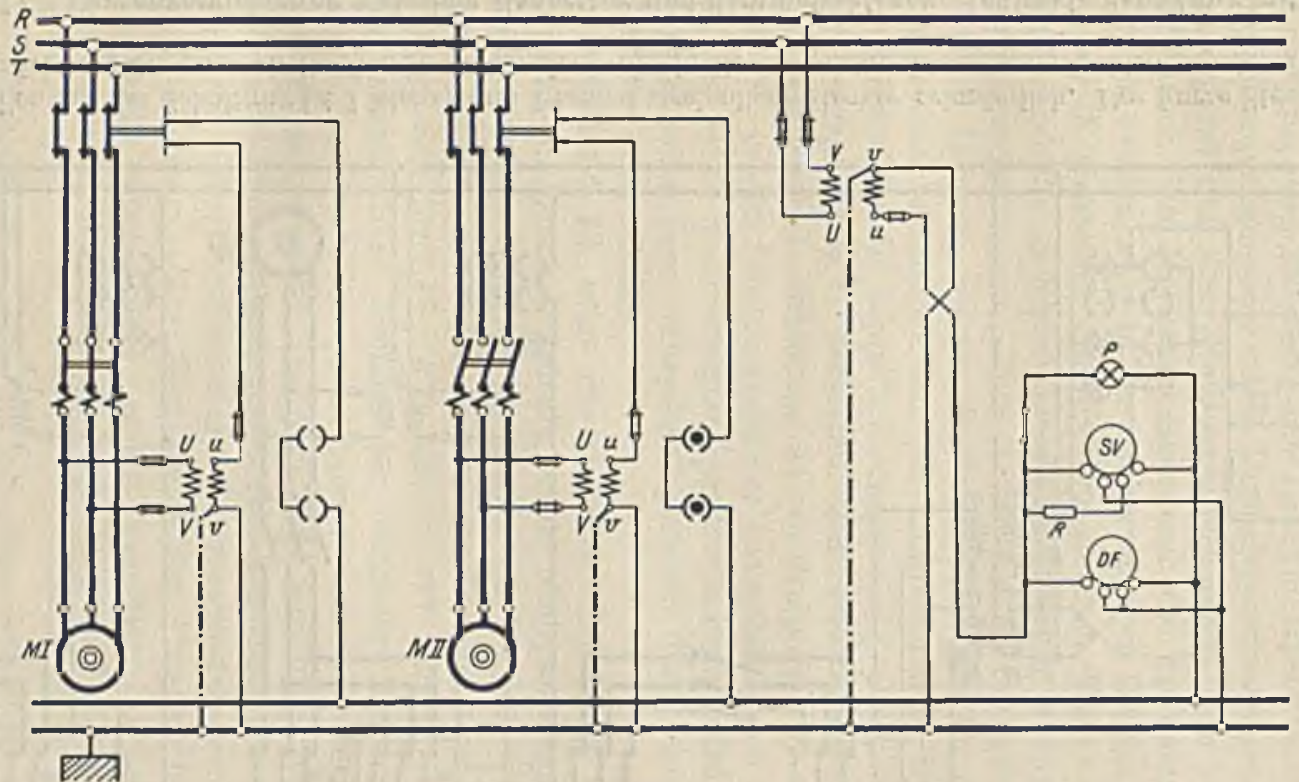

Für die Bedienung der Schaltung ist nur 1 kurzer zweipoliger Stecker erforderlich, der in die Stockeinrichtung der zuzuschaltenden Maschine eingeführt wird.

Schaltbild 10. Phasenrergleichung zwischen Generator und Sammelschienen. Indirckte Schaltung mit Summenspannungsmesser und Phasenlampe in IIellschaltuug. (Bild 72.) 


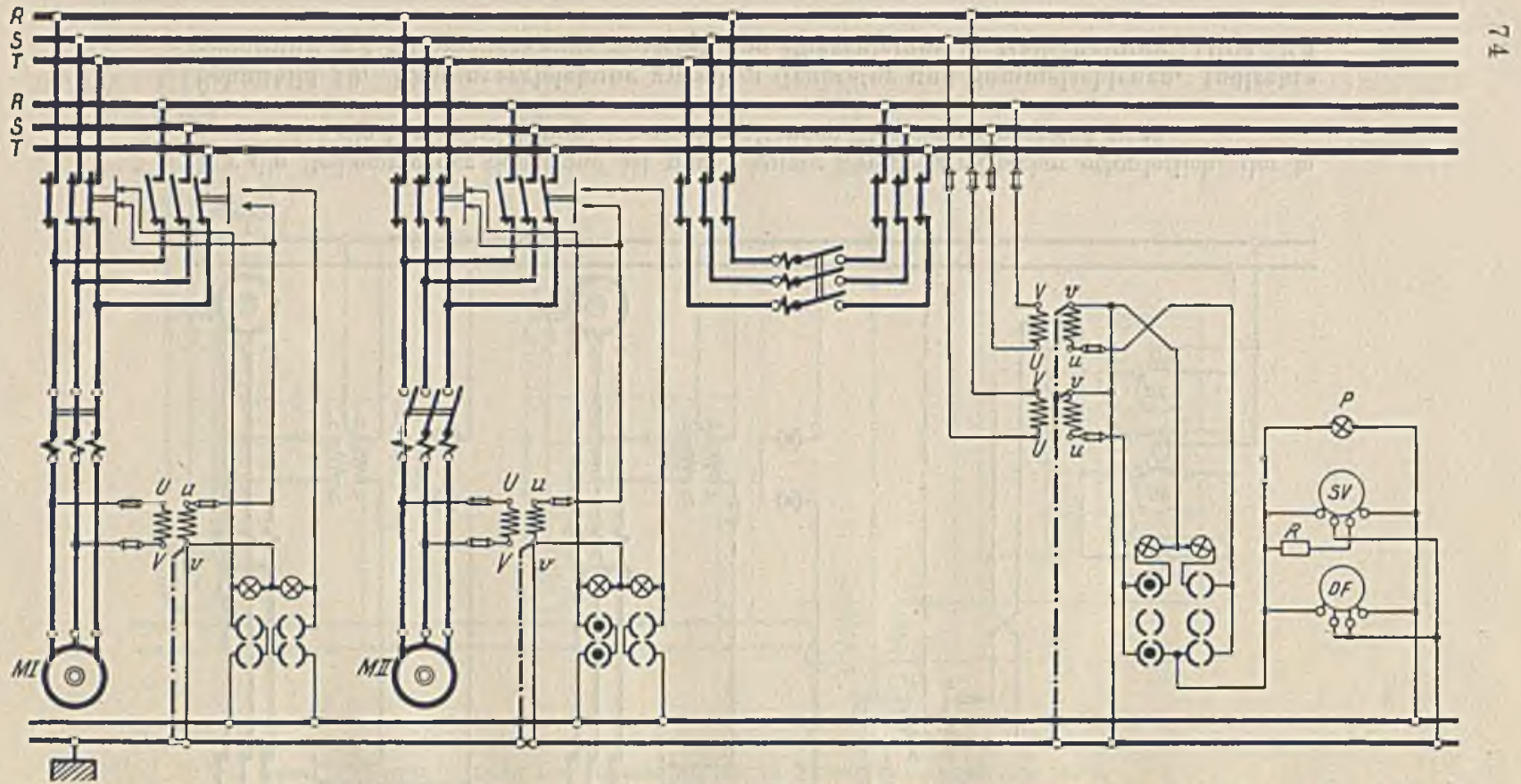

Für die Bedienung der Schaltung ist 1 kurzer und 1 langer zweipoliger Stecker erforderlich. Der kurze Stecker wird in die Steckeinrichtung dor zuzuschaltenden Maschine, der lange in die des gewünschten Sammelschienonsystems eingefübrt.

Schaltbild 11. Phasenvergleichung zwischen Generator und Sammelschienen. Indirekte Schaltung mit Summenspannungsmesser und Phasenlampe in Hellschaltuug; Pür Doppelsammelschienen. (Bild 73.) 


\section{c. Schaltungon mit Lampennpparat.}

Schaltbild 12 zeigt eine direkte Schaltung mit Lampenapparat in Dunkelschaltung und mit Nullspannungsmesser für Spannungen bis etwa 250 Volt. Der Instrumentsatz besteht aus einem Doppelfrequenzmesser, einem Doppelspannungsmesser, dem Lampenapparat und dem Nullspannungsmesser. Um zu vermeiden, daß durch den Nullspannungsmesser die Spamungen ungleich verteilt werden, sind in die beiden anderen Zweige Ersatzwiderstände $R$ einzuschalten, die den gleichen Eigenverbrauch und annähernd die gleiche Widerstandsänderung aufweisen wie der Nullspannungsmesser. Man verwendet hierzu zweckmäßig die gleichen Lampen, wie sie als Vorschaltlampen für den Nullspannungsmesser benutzt werden. Alle Lampen und der Nullspannungsmesser sind für die doppelte Sternspannung, also für das 1,15 fache der Netzspannung zu bemessen. Zur betriebsmäBigen Bedienung der Parallelschalteinrichtung ist ein dreipoliger Stecker erforderlich, der in den Steckkontakt der zuzuschaltenden Maschino eingeführt wird. Nach erfolgter. Synchronisierung wird der Lampen. apparat nebst dem Nullspannungsmesser durch einen dreipoligen Schalter ausgeschaltet.

Schaltbild 13 zeigt die indirekte Schaltung mit Lampenapparat in Dunkelschaltung und Nullspannungsmesser. Der Instrumentsatz ist genau der gleiche wie bei dem vorher beschriebenen Schaltbild 12, jedoch sind die Lampen und der Nullspannungsmesser für $1,15 \times 110$, also für 127 Volt zu bemessen. Um eine einwandfreie Erclung der Sekundärscite der Spannungswandler zu ermöglichen und dabei trotzdem die für den Lampenapparat erforderliche Trennung aller Leitungen aufrecht zu erhalten, ist hinter dem Sammelschienen-Spannungswandler ein besonderer Isoliertransformator $J T$ eingeschaltct, der im Verhältnis 1: I übersetzt. Zur Betätigung der Parallelschalteinrichtung ist für die ganze Anlage nur ein zweipoliger Stecker erforderlich, der in den Steckkontakt der zuzuschaltenden Maschine eingeführt wird.

Schaltbild 14 zeigt die indirekte Schaltung mit Lampenapparat in Hellschaltung und Summenspannungsmesser. Die Lampen des Lampenapparates sind wieder für $1,15 \times 110=127$ Volt zu bemessen. Als Summenspannungsmesser wird ein Doppelspannungsmesser mit zwei Meßwerken verwendet (vgl. S. 36). 
Um es zu erreichen, daß die beiden Zeiger des Instruments bei Phasengleichheit auf dem gleichen Wert 110 Volt stehen, wird vor das als Summenspannungsmesser geschaltete Meßwerk ein besonderer Vorwiderstand $R$ gelegt, der $15 \%$ der Spannung vernichtet. $\mathrm{Da}$ der Eigenverbrauch des Summenspannungsmessers sehr klein ist, sind . Ersatzwiderstände in den beiden anderen Phasen nicht crforderlich. Die für die Hellschaltung erforderliche Ubberkreuzung der Leitungen ist auf der Sekundärseite des Isoliertransformators $J T$ vorgenommen worden. Im übrigen ist die Schaltung die gleiche wie bei dem vorhergehenden Schaltbild 13. Zur Bedienung der ganzen Anlage ist ein zweipoliger Stecker erforderlich, der in den Steckkontakt der zuzuschaltenden Maschine eingeführt wird.

\section{d. Sehaltungen mit Synchronoskop.}

Sehaltbild 15 zeigt die direkte Schaltung mit SiemensSynchronoskop und Phasenlampen in Dunkelschaltung. Die Schaltung ist für Spannungen bis 250 Volt bestimmt. Der Instrumentsatz besteht aus einem Doppelfrequenzmesser, einem Doppelspannungsmesser, einem Synchronoskop und zwei Phasenlampen. Das Synchronoskop und die Phasenlampen sind für die einfache Netzspannung zu bemessen. Vor das Synchronoskop muß stets ein besonderer Ausschalter eingebaut werden, da der Apparat erst dann eingeschaltet werden darf, wenn die Frequenzabweichungen zwischen Generator und Netz nicht mehr als 5\% betragen. Zur Bedienung der ganzen Anlage ist nur ein zweipoliger Stecker erforderlich, der in den Steckkontakt der zuzuschaltenden Maschine eingeführt wird.

Schaltbild 16 zeigt die indirekte Schaltung mit Siemens. Synchronoskop und Phasenlampe in Dunkelschaltung. Da alle Meßwandler durch die Erdleitung einpolig verbunden sind, ist nur eine Phasenlampe ciforderlich, die für die doppelte Sekundärspannung der Spannungswandler, also für $2 \times 110$ Volt, zu bemessen ist. Das Synchronoskop ist dagegen nur für 110 Volt zu wählen, da in ihm nur die beiden Einzelspannungen auftreten. Der Ausschalter vor dem Synchronoskop darf nur zweipolig sein, da die an Erde liegenden Leitungen nicht unterbrochen werden dürfen. Die an den Trennschaltern angebrachten Hilfskontakte verhüten, daß abgeschaltete Maschinen bei versehentlichen falschen 
Stöpseln durch Rücktransformierung des zugehörigen Spannungswandlers unter Spannung gesetzt werden. Zur betriebsmäßigen Bedienung der ganzen Anlage ist nur ein zweipoliger Stecker erforderlich, der in den Stecklontakt der zuzuschaltenden Maschine eingeführt wird.

Schaltbild 17 zeigt die indirekte Schaltung mit Siemens-Synchronoskop und Phasenlampe in Dunkelschaltung bei Doppelsammelschienen. Entsprechend den zwei Sammelschienensystemen sind zwei Satz Sammelschienen-Transformatoren erforderlich, die mittels zweier Steckeinrichtungen wahlweise auf den Instrumentsatz geschaltet werden. Beim Einführen des Sammelschienensteckers leuchtet stets eine dem gewählten Sammelschienensystem entsprechende farbige Signallampe auf. An den Steckvorrichtungen der Maschinen sind cbenfalls farbige Signallampen angebracht, die aber durch die Hilfskontalste an den Trennschaltern eingeschaltet werden. Man kann daher an der Farbe der brennenden Signallampen stets von vornherein erkennen, welche Trennschalter eingelegt und welche Sammelschienen an die Meßeinrichtung angeschlossen sind. Fin versehentliches Schalten auf falschc Sammelschienen ist somit kaum noch möglich. Gleichzeitig mit der Signalgebung erfüllen die Hilfskontakte noch den gleichen Zweck wie bei Schaltbild 16. Zur Bedienung der ganzen Anlage ist ein kurzer zweipoliger und ein dreipoliger Stecker erforderlich. Soll eine Maschine neu in Betrieb genommen werden, so sind die Stecker so zu stecken, daß an der Maschinen- und an der Sammelschienen-Steckeinrichtung gleichfarbige Lampen leuchten. Sollen dagegen verschiedene Sammelschienensysteme parallel geschaltet werden, so vergleicht man eine an dem einen Sammelschienensystem bereits laufende Naschine mit dem anderen Sammelschienensystem. In diesem Falle müssen daher verschiedenfarbige Lampen leuchten. Etwaige von einem fremden Kraftwerk kommende Speiseleitungen werden in der gleichen Weise geschaltet wie dic einzelnen Maschinen.

Die Schaltungen 16 und 17 können noch wesentlich vervollkommet werden, wenn man die Phasenlampe an einen Umkehrtransformator anschließt (vgl. S. 12), so daß sie bei Phasengleich. heit aufleuchtet. Der Instrumentsatz entspricht dann genau der auf Bild 58 dargestellten Anordnung. 


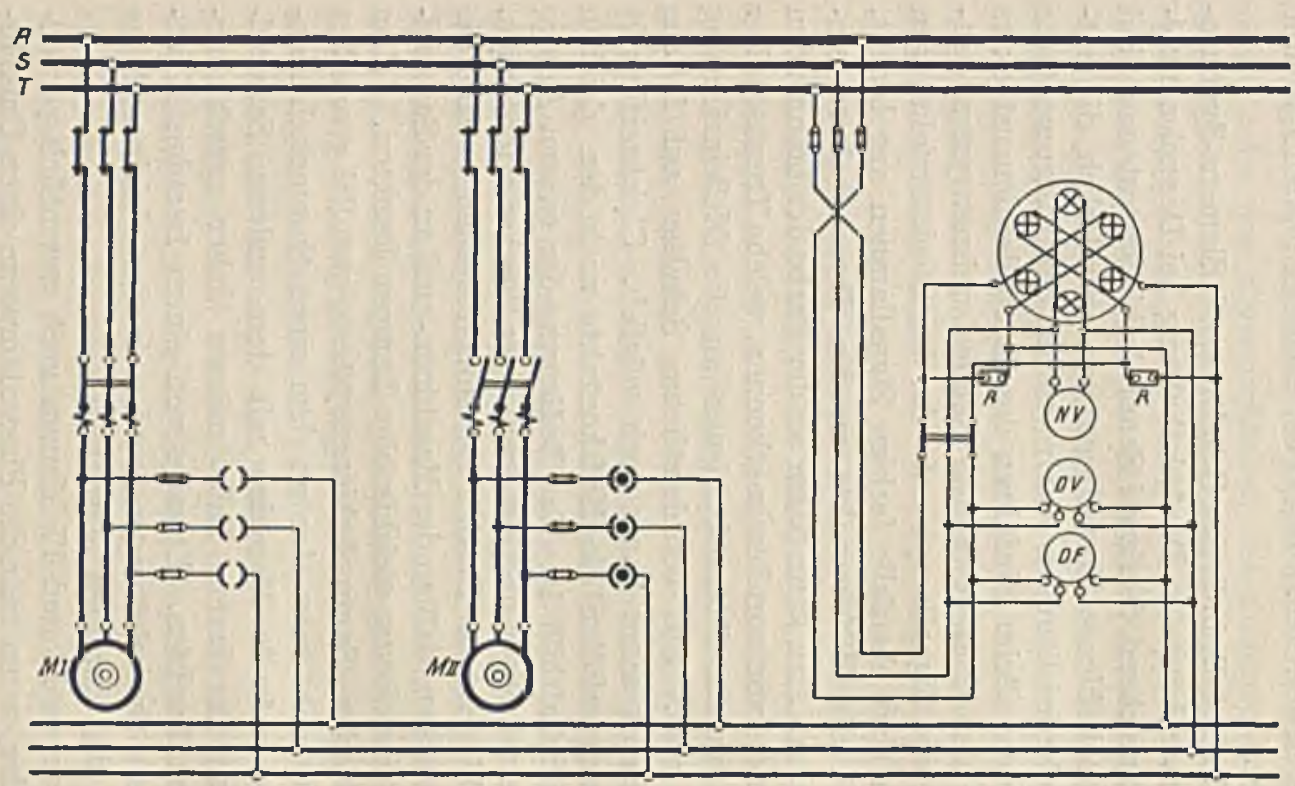

Für die Bedienung der Schaltung ist nur 1 dreipoliger Stecker erforderlich, der in die Steckeinrichtung der zuzuschaltenden Maschine eingeführt wird.

Schaltbild 12. Phasenvergleichung zwischen Generator und Sammelschienen. Direkte Sehaltung mit Lamponapparat in Dunkelschaltung und Nullspannungsmesser. (Bild 74.) 


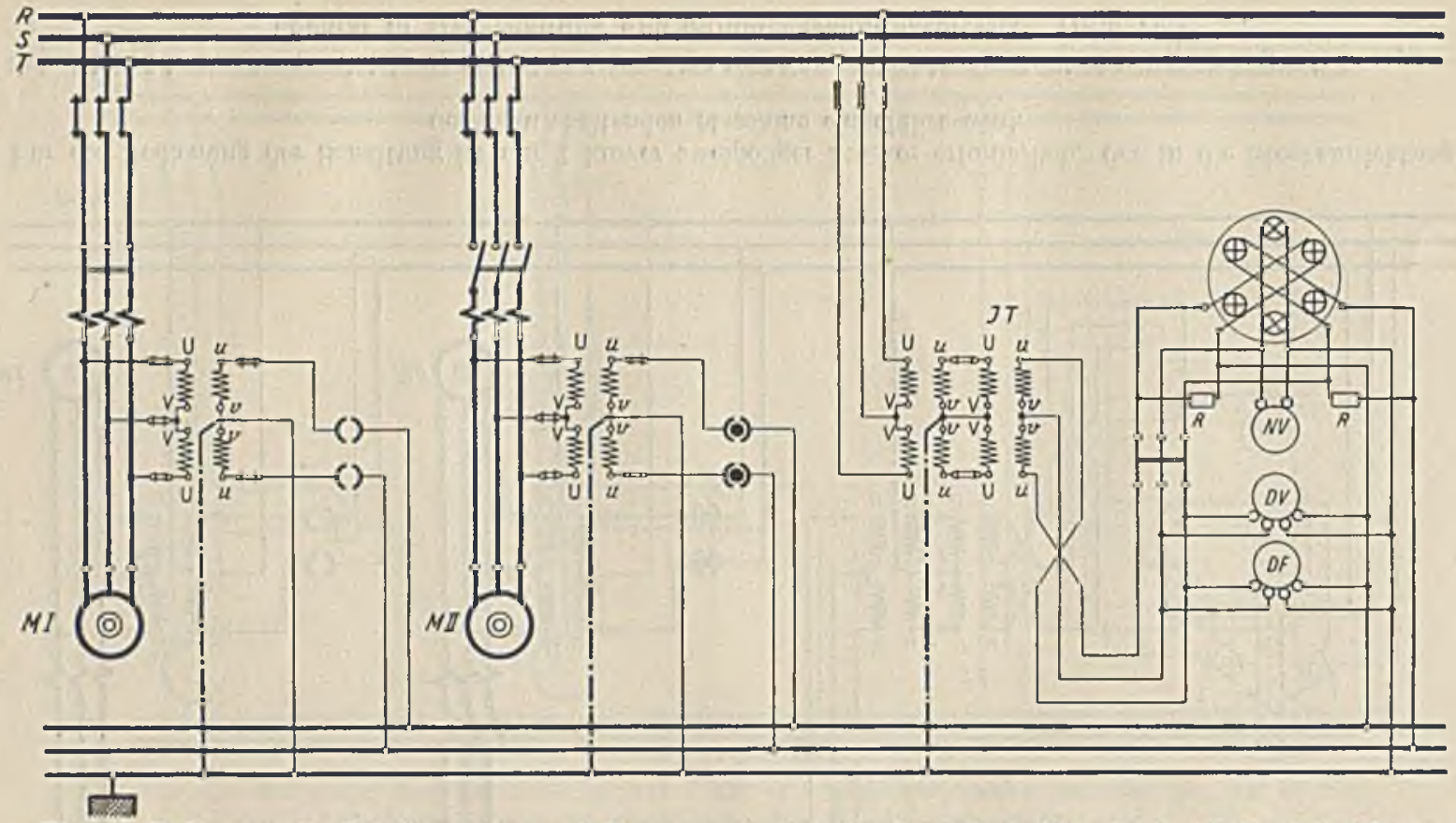

Für dio Bedienung der Schaltung ist nur 1 kurzer zweipoliger Stecker erforderlich, der in die Steckeinrichtung der zuzuschaltenden Maschine eingoführt wird.

Schaltbild 13. Phasenvergleichung zwischen Generator und Sammelschienen. Indirekte Schaltung mit Lampenapparat in Dunkelschaltung und Nullspannungsmesser. (Bild 75.) 


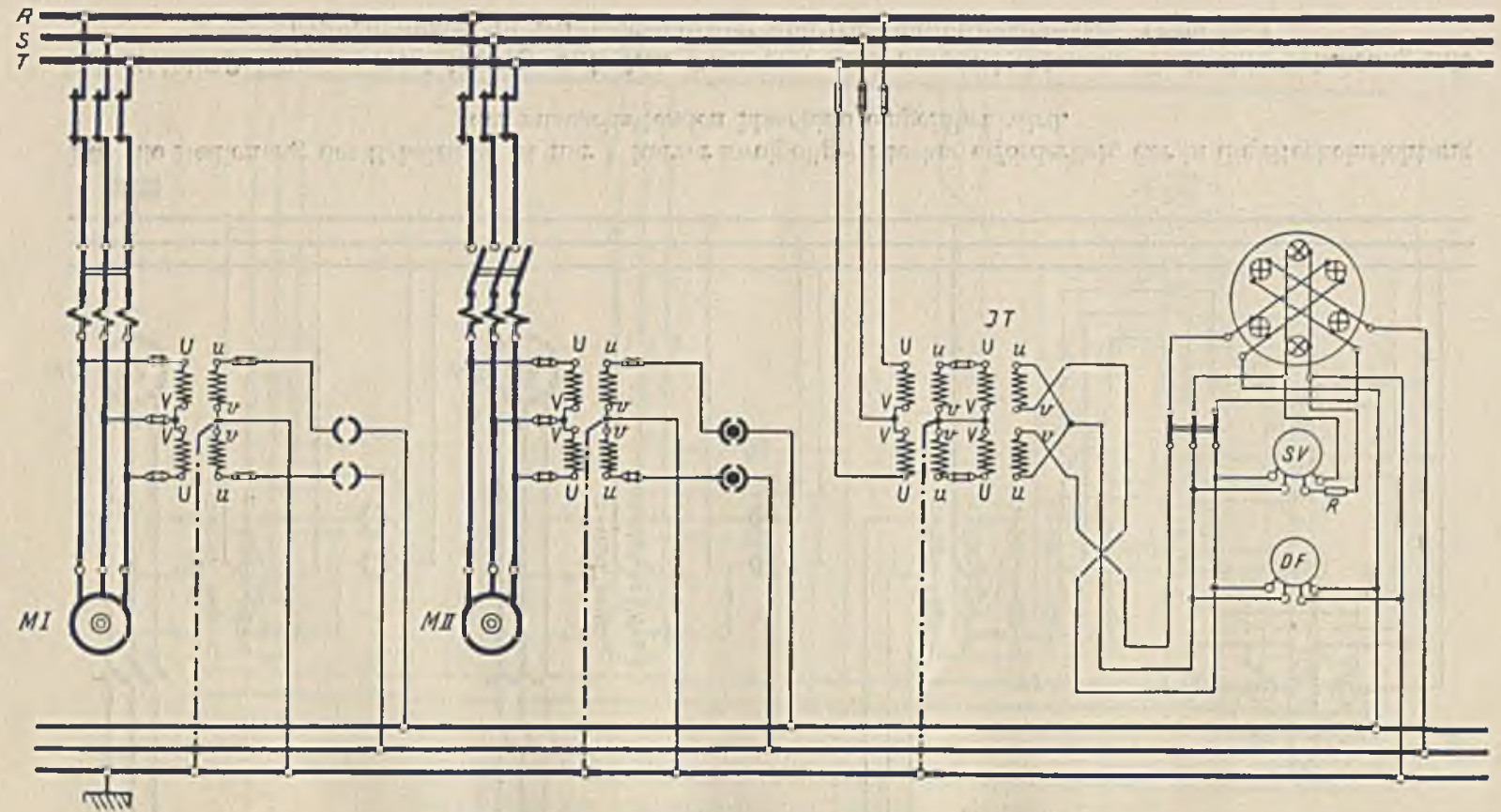

Für die Bedienung der Schaltung ist nur I kurzer zweipoliger Stocker erforderlich, der in dio Steckeinrichtung der zuzuschaltenden Maschine eingeführt wird.

Schaltbild 14. Phasenvergleichung zwischen Generator und Sammelsehienon. Indirekte Schaltung mit Lampenapparat in IIellschaltung und Summenspannungsmesser. (Bild 76.) 


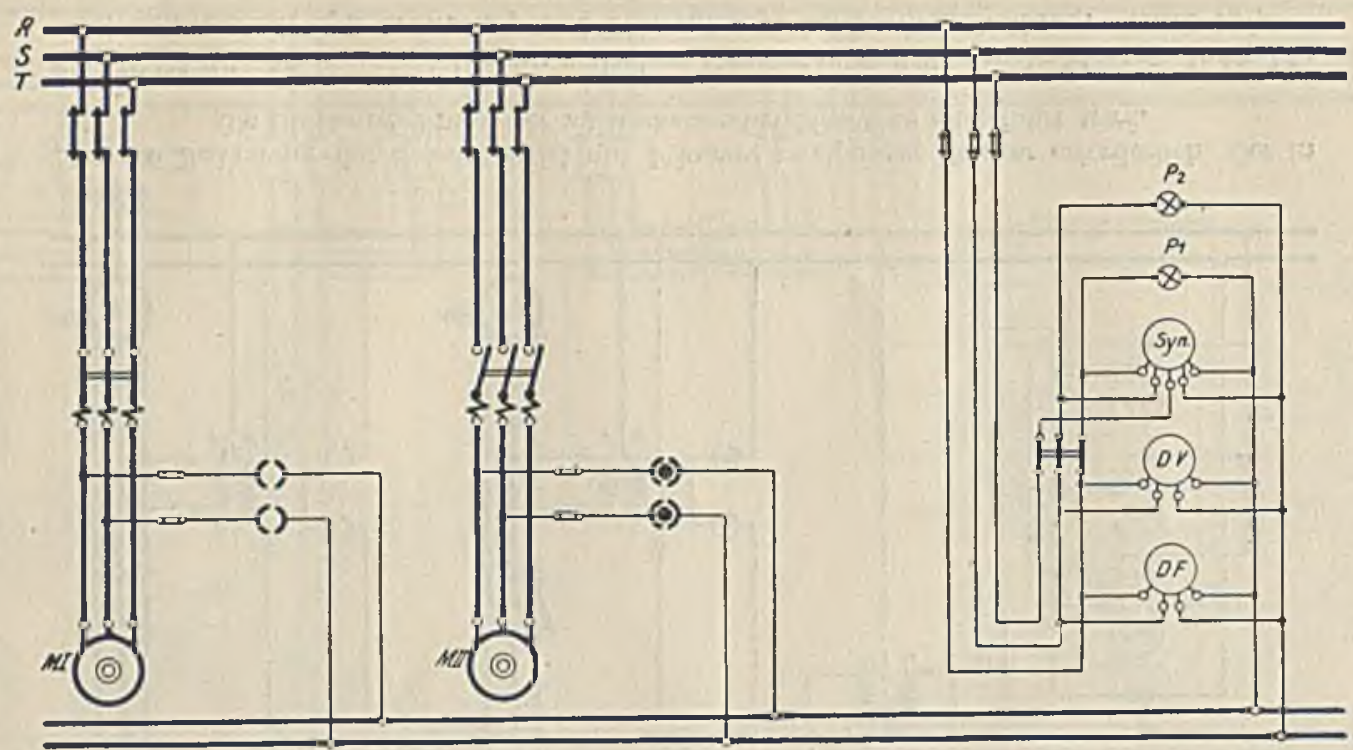

Für die Bedienung der Schaltung ist nur 1 kurzer zwoipoliger Stecker erforderlich, der in die Steckeinrichtung der zuzuschaltenden Maschine eingeführt wird.

Schaltbild 15. Phasenvergleichung zwischen Generator und Sammelschienen. Direkte Schaltung mit Synchronoskop und Phasenlampen in Dunkelschsltung. (Bild 77.) 


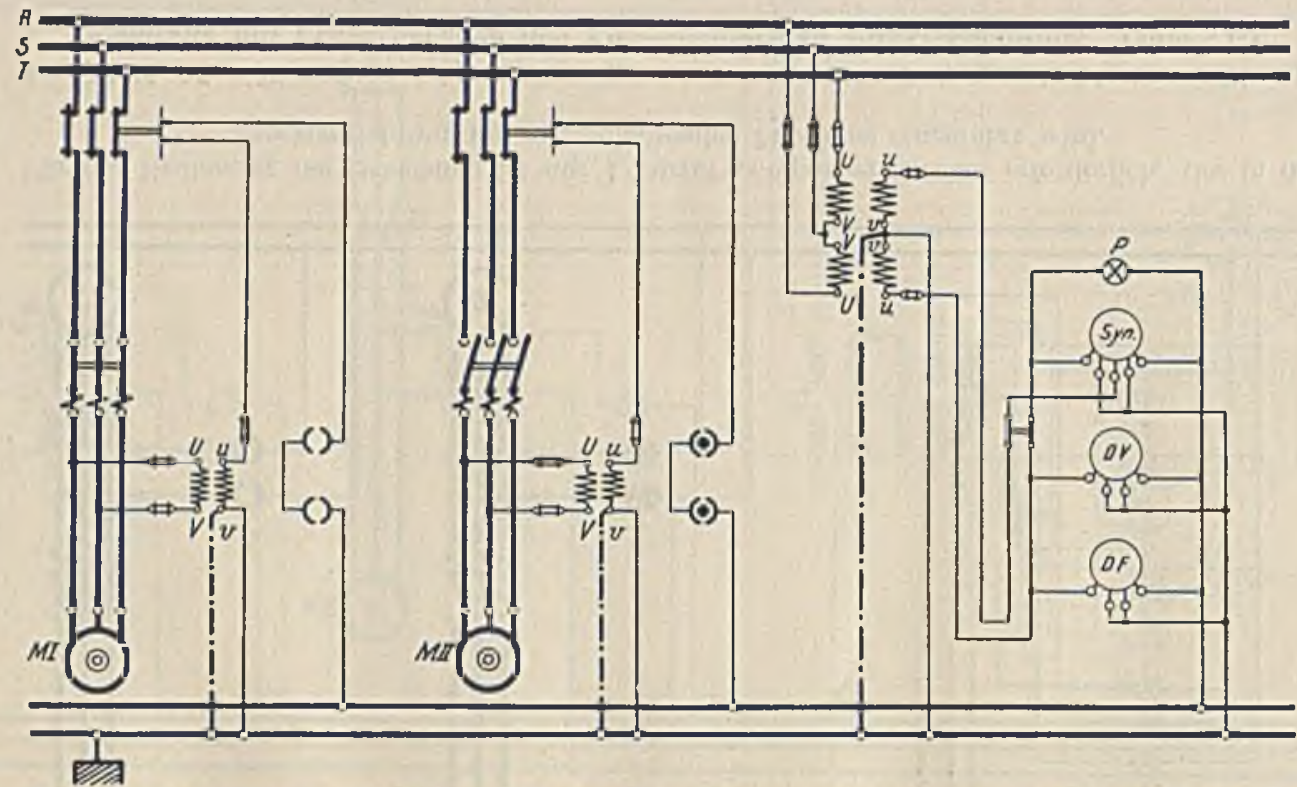

Für die Bedienung der Schaltung ist nur 1 kurzer zweipoliger Stecker erforderlich, der in die Steckeinrichtung der zuzuschaltenden Maschine eingeführt wird.

Schaltbild 16. Phasenvergleichung zwischen Generator und Sammelschienen. Indirekto Schaltung mit Synchronoskop und Phasenlampe in Dunkelschaltung. (Bild 78.) 


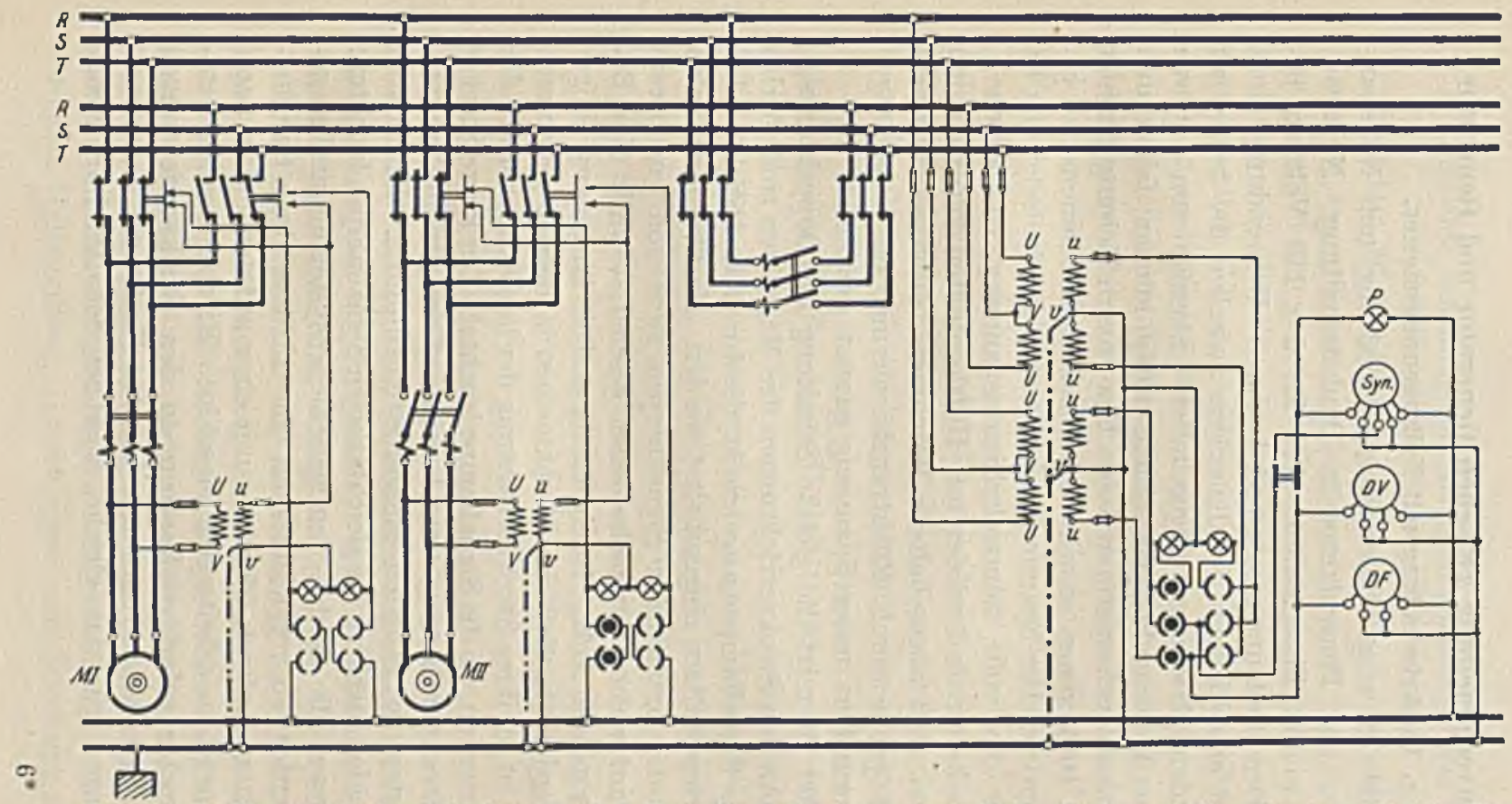

Für die Bedienung ist 1 zweipoliger und 1 dreipoliger Stecker erforderlich. Der zweipolige Stecker wird in die Steckoinrichtung der zuzuschaltenden Maschine, der dreipolige in die der gewünschten Sammelschienen eingeführt.

Schaltbild 17. Phasenvergleichung zwischen Generator und Sammelschienen. Indirekto Schaltung mit Synchronoskop und Phasenlampe in Dunkelschaltung; fïr Doppelsammelschienen. (Bild 79.) 


\section{Phasenvergleichung zwischen Generator und Generator.}

\section{n. Dunkelschnltung mit Nullspannungsmesser.}

Schaltbild 18 zeigt die indirekte Schaltung mit Nullspannungsmesser und Phasenlampe in Dunkclschaltung. Nullspannungsmesser und Phasenlampe sind für $2 \times 110$ Volt zu bemessen. Der Instrumentsatz wird durch Verwendung eines kurzen und eines langen Schaltsteckers zwischen die beiden zu vergleichenden Maschinen eingeschaltet. Etwaige von anderen Kraftwerken kommende Speiseleitungen $(S p)$ kömnen, falls durch die Maschinentransformatoren keine Phasenverschiebung bedingt ist (vgl. S. 106), genau so wie die einzelnen Maschinensätze geschaltet werden. Der Spannungswandler für die Speiseleitung ist jedoch für die volle Sammelschienenspannung zu bemessen. Die Trennschalter sind wieder mit Hilfskontakten versehen, um zu verhüten, daß abgeschaltete Maschinen bei versehentlichem falschen Stöpseln durch Rücktransformierung des zugehörigen Spannungswandlers unter Spannung gesetzt werden.

Schalthild 19 zeigt die gleiche Schaltung für Doppelsammel. schienen. An den Steckvorrichtungen der Maschinen sind hierbei noch farbige Signallampen angebracht, die durch die Hilfskontakte an den Trennschaltern eingeschaltet werden. Man kann daher an der Farbe der brennenden Signallampen stets von vornherein crkennen, auf wclche Sammelschienen geschaltet wird. Zur Bedienung der ganzen Anlage ist ein kurzer und ein langer zweipoliger Stecker erforderlich. Soll eine Maschine neu in Betrieb genommen werden, so ist an ihrer Steckvorrichtung der lange Stecker auf der Seite einzustecken, wo die Signallampe leuchtet. Der kurze Stecker ist in die Steckvorrichtung ciner bereits auf das gleiche Sammelschienensystem arbeitenden Maschine einzuführen. Es müssen dann bei beiden Steckern die gleichfarbigen Signallampen leuchten. Sollen dagegen die beiden Sammelschienensysteme parallel geschaltet werden, so vergleicht man eine an dem einen Sammelschienensystem laufende Maschine mit einer auf das andere Sammelschienensystem arbeitenden Maschine. Hierbei werden demgemäß verschiedenfarbige Lampen an den Steckvorrichtungen leuchten. Für etwaige von einem fremden Kraftwerk kommende Speiseleitungen gilt das gleiche, was bei Schaltbild 18 gesagt wurde. 


\section{b. Gemisclito Schaltung mit Nullspamungsmesser und Umkehr- transformator Piir die Pliasenlampe.}

Schaltbild 20 zeigt eine gemischte Schaltung mit dem vom Verfasser angegebenen Umkehrtransformator (vgl, S. 13). Diese unterscheidet sich von der im Schaltbild 18 angegebenen Schaltung dadurch, daß die Phasenlampe an cinen besonderen Umkehrtransformator $U T$ angeschlossen ist. Durch den. Umkehrtransformator wird ummittelbar am Instrumentsatz die eine der beiden zu vergleichenden Spannungen um $180^{\circ}$ gedreht, so daß die Phasenlampe trotz der unverändert weiterbestehenden Dunkelschaltung der Generatorenschaltanlage in Hellschaltung liegt. Die Phasenlampe gibt dann durch ihr Aufleuchten im richtigen Augenblick das Achtungssignal für das Parallelschalten. Das Schalten wird demnach viel sicherer als bei der Dunkelschaltung von statten gehen (vgl. S. I7). Weiterhin wird hierbei noch die Betriebssicherheit der Schaltvorrichtung wesentlich erhöht, da das Achtungssignal cben nur bei vollkommen intakter Schaltung erfolgen kann. Die durch den Umkehrtransformator entstehenden Mchrkosten sind gegenüber diesen Vorteilen belanglos, da der Umkehrtransformator in diesem Falle ein ganz kleiner, billiger Transformator sein kann. Die Übersetzung des Umkehrtransformators ist stets $1: 1 \mathrm{zu}$ wählen, also $110: 110$ Volt. Seine Leistung braucht nur halb so groß zu scin als der Verbrauch der Phasenlampe.

Die Schaltung kann in gleicher Weise auch für Doppelsammelschienen benutzt werden. Die Maschinenschaltung ist dann genau wie im Schaltbild 19 auszuführen.

\section{c. Umkehrsehaltung mit Summenspannungsmesser.}

Schaltbild 21 zeigt die Verwendung des Umkehrtransformators für den Anschluß der Phasenlampe und cines Summenspannungsmessers. Die Schaltung ermöglicht also die Verwendung einer vollständigen Apparatur für Hellschaltung im Anschluß an eine bestehende Dunkelschaltung der Generatorenschaltanlage. Die von der Generatorenschaltanlage erzeugte Differenzspannung wird hierbei unmittelbar am Instrumentsatz durch den dort angebrachten Umkehrtransformator in eine Summenspannung verwandelt, so daß die gleiche Wirkung erzielt wird wie bei einer normalen Hellschaltung. Durch diese neue Schaltung ist es nun: 
mehr möglich geworden, für die Generatorenschaltanlage stets die gleiche einfache Schaltweise der Dunkelschaltung zu wählen, ganz unabhängig davon, ob man für den Instrumentsatz Dunkeloder Hellschaltung anwenden will (vgl. S. 14). Alle Schwierigkeiten, die die Verwendung der Hellschaltung bei der wahlweisen Schaltung von Generator zu Generator unmöglich machen, sind hiermit behoben, da die Schaltung für alle Generatoren genau die gleiche ist und alle Spannungswandler einschließlich des Umkehrtransformators einpolig verbunden und in normaler Weise geerdet werden können. Es besteht demgemäß kein Hinderungsgrund mehr, die hinsichtlich der Betriebssicherheit überlegeno Hellschaltung an Stelle der Dunkelschaltung anzuwenden. Die Kosten für den zusätzlichen Umkehrtransformator sind auch hier gegenüber den erreichten Vorteilen belanglos, da ein Meßwandler kleinster Type genügt, der für 110 Volt bemessen sein muß und im Verhältnis $1: 1$ übersetzt.

Schaltbild 22 ist eine Weiterentwicklung des Schaltbildes 21. Der Doppelspannungsmesser wird hierbei in ähnlicher Weise wie im Schaltbild 8 durch einen Umschalter einmal als Maschinenspannungsmesser und das andere $\mathrm{Mal}$ als Summenspannungsmesser geschaltet. Steht der hierzu erforderliche dreipolige Umschalter in der Schaltstellung $M$, so dient das an die rechten Instrumentklemmen angeschlossene Meßwerk als Maschinenspannungsmesser. Der Stromkreis der Phasenlampe ist hierbei unterbrochen. Steht der Umschalter dagegen in der Schaltstellung $S$, so arbeitet das Instrument als Summenspannungsmesser. Vor seinem Meßwerk liegt hierbei der Vorwiderstand $R$ und parallel zu der Reihenschaltung die Phasenlampe $P$. Die Phasenlampe und der Umkehrtransformator sind jetzt eingeschaltet, so daß die Phasenlampe periodisch aufleuchtet und bei Phasengleichheit durch ihr dauerndes Leuchten das Achtungssignal zum Parallelschalten gibt. Auch diese beiden Schaltungen können ohne weiteres für Anlagen mit Doppelsammelschienen benutzt werden, wenn man die Maschinen nach Schaltbild 19 schaltet.

\section{d. Schaltungen mit Synchronoskop.}

Schaitbild 23 stellt eine indirekte Schaltung mit SiemensSynchronoskop und Phasenlampe für Dunkelschaltung dar. Der Instrumentsatz ist genau der gleiche wie bei Schaltbild 16. Das 
Synchronoskop ist für 110 Volt, dic Phasenlampe für $2 \times 110$ Volt zu bemessen. Die einzelnen Maschinensätze sind vollkommen gleich geschaltet. Die für das richtige Arbeiten des Synchronoskops erforderliche Verschiedenheit der Schaltungen für dio bereits la ufende und die zuzuschaltende Maschine wird durch verschieden. artige Stecker erreicht. Für die zuzuschaltende Maschine wird ein einpoliger Stecker benutzt, der nur den einen Spannungswandler einschaltet, während für die bereits laufende Maschine ein langer zweipoliger Stecker verwendet wird, der beide Spannungswandler und damit den für den Betrieb des Synchronoskops erforderlichen Drehstrom einschaltet. An den Trennschaltern der Maschinen sind wieder Hilfskontakte angebracht, die die Parallelschalteinrichtung bei ausgeschalteten Trennschaltern unterbrechen. Für Speiseleitungen aus fremden Kraftwerken ist nur I Spannungswandler erforderlich, der allerdings für die Oberspannung bemessen sein muß. Die. Schaltung setzt jedoch voraus, daß zwischen Unterspannung und Oberspannung keine Phasenverschiebung vorhanden ist (vgl. S. 106).

Schaltbild 24 zeigt die gleiche Schaltung für Doppelsammelschienen. An den Steckvorrichtungen der Maschinen sind hierbei wieder farbige Signallampen angebracht, die durch die Hilfskontakte an den Trennschaltern eingeschaltet werden. Man kann daher an der Farbe der brennenden Signallampen stets von vornherein erkennen, auf welche Sammelschienen geschaltet wird. Gleichzeitig verhüten die Signalkontakte noch ein versehentliches Unterspannungsetzen der Maschinen. Zur Bedienung der ganzen Schaltanlage dient ebenso wie bei Schaltbild 23 ein einpoliger und ein langer zweipoliger Stecker. Soll eine Maschine neu in Betrieb genommen werden, so ist an der zugehörigen Steckvorrichtung der einpolige Stecker auf der Seite einzuführen, auf der die Signallampe leuchtet. Der zweipolige Stecker ist dann in die Steckvorrichtung einer auf das gleiche Sammelschienensystem bereits arbeitenden Maschine einzuführen. Es müssen dann über den Steckern gleichfarbige Signallampen brennen. Sollen dagegen verschiedene Sammelschienensysteme parallel geschaltet werden, so vergleicht man eine auf das eine Sammelschienensystem bereits arbeitende Maschine mit einer auf das andere Sammelschienensystem arbeitenden Maschine. Man führt den einpoligen Stecker dann bei der Maschine ein, die man regulieren will und den zwei- 


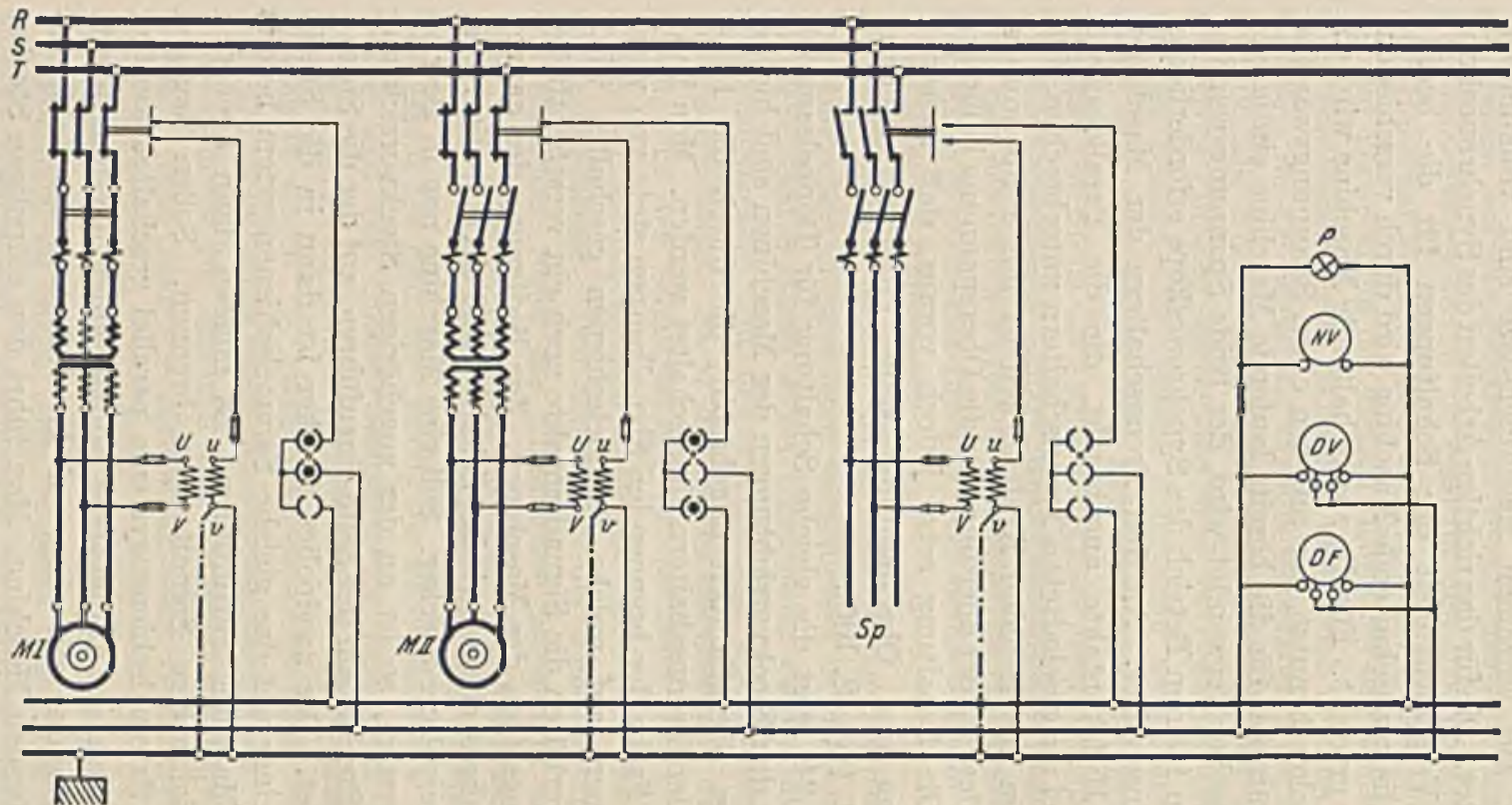

Für die Bedienung der Schaltung ist 1 kurzer zweipoliger Stecker mit Sperrstift und 1 langer zweipoliger Stecker erforderlich. Der kurze Stecker wird in die Steckeinrichtung einer bereits laufenden, der lange in die der zuzuschaltenden Maschine eingeführt.

Schaltbild 18. Phasenvergleichung zwischen Generator und Generator. Schaltung mit Nullspannungsmesser und Phasenlampe in Dunkelschaltung. (Bild 80.) 


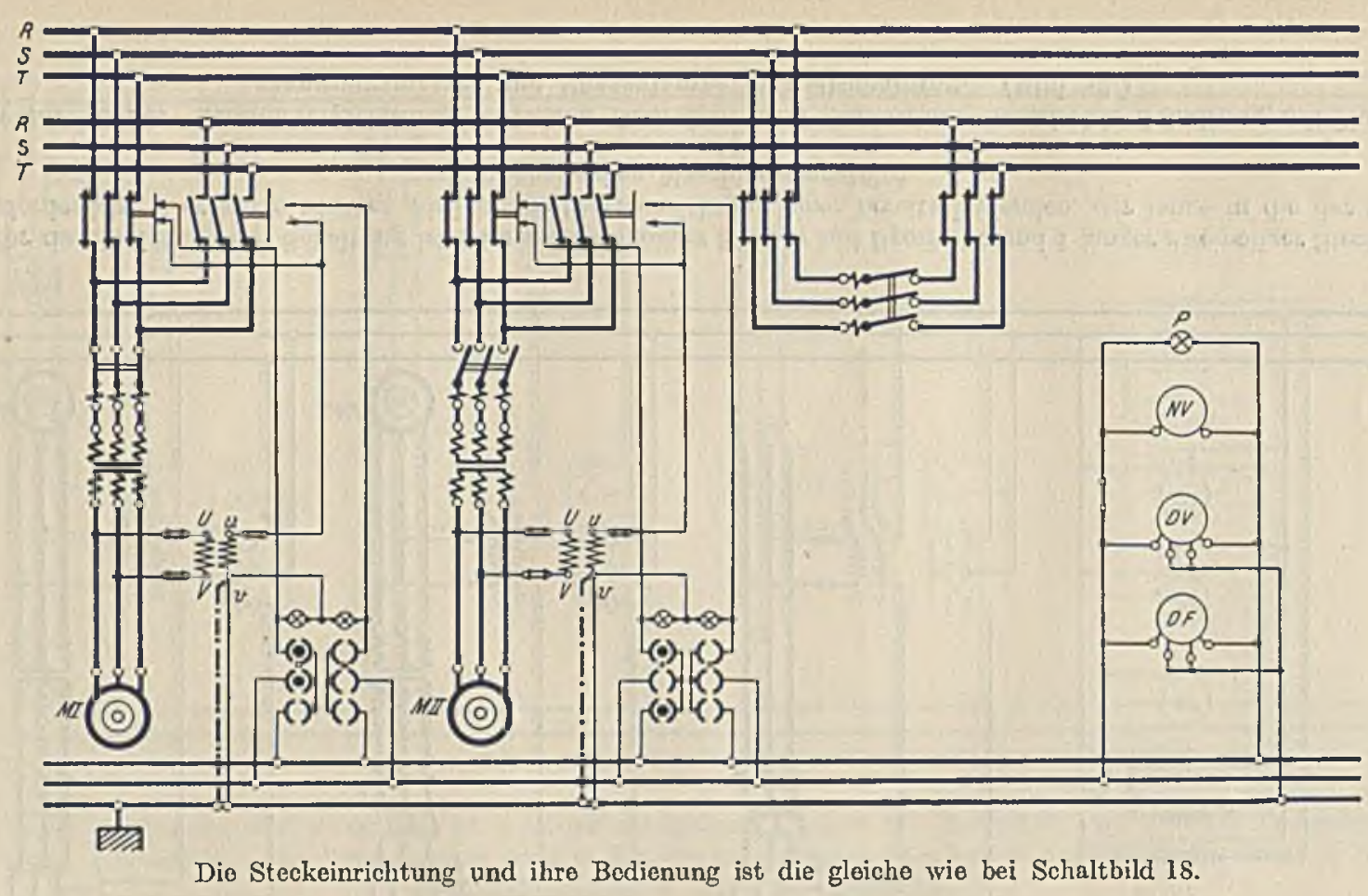

Schaltbild 19. Phasenvergleichung zwischen Generator und Generator. Schaltung mit Nullspanuungsmesser und Phasenlampe in Dunkelschaltung; für Doppelsammelsehienen. (Bild S1.) 


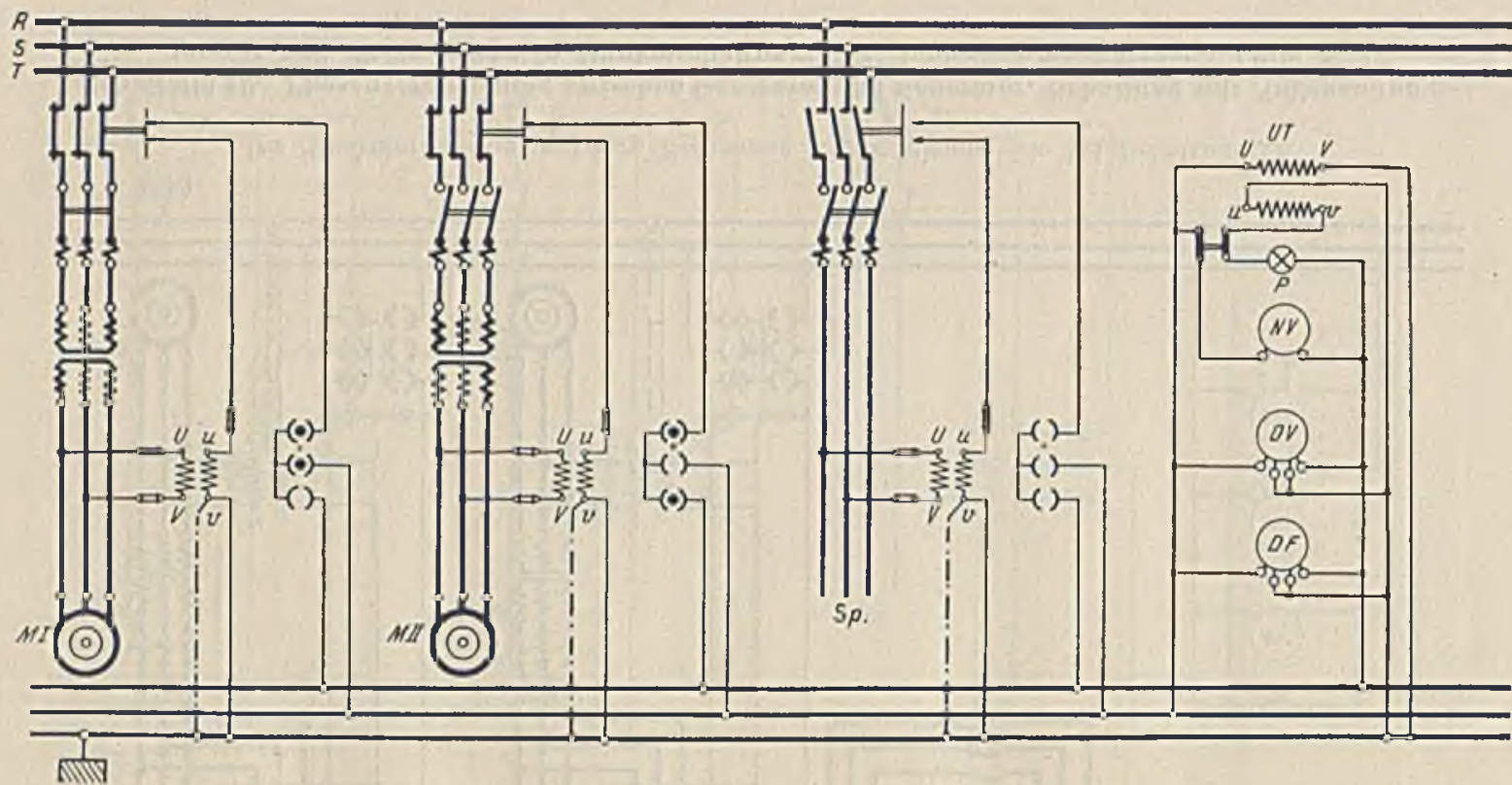

Für die Bedionung der Sehaltung ist 1 kurzer zwoipoliger Steckor mit Sperrstift und 1 langer zweipoliger Stecker orforderlich. Der kurze Stecker wird in dio Steckeinrichtung einer bereits laufenden, der lange in die der zuzuschaltenden Maschine eingeführt.

Schaltbild 20. Phasenvergleichung zwisehen Generator und Generator. Gemisehte Sehaltung mit Nullspannungsmesser und Phasenlampe in Mellschaltung. (Bild 82.) 


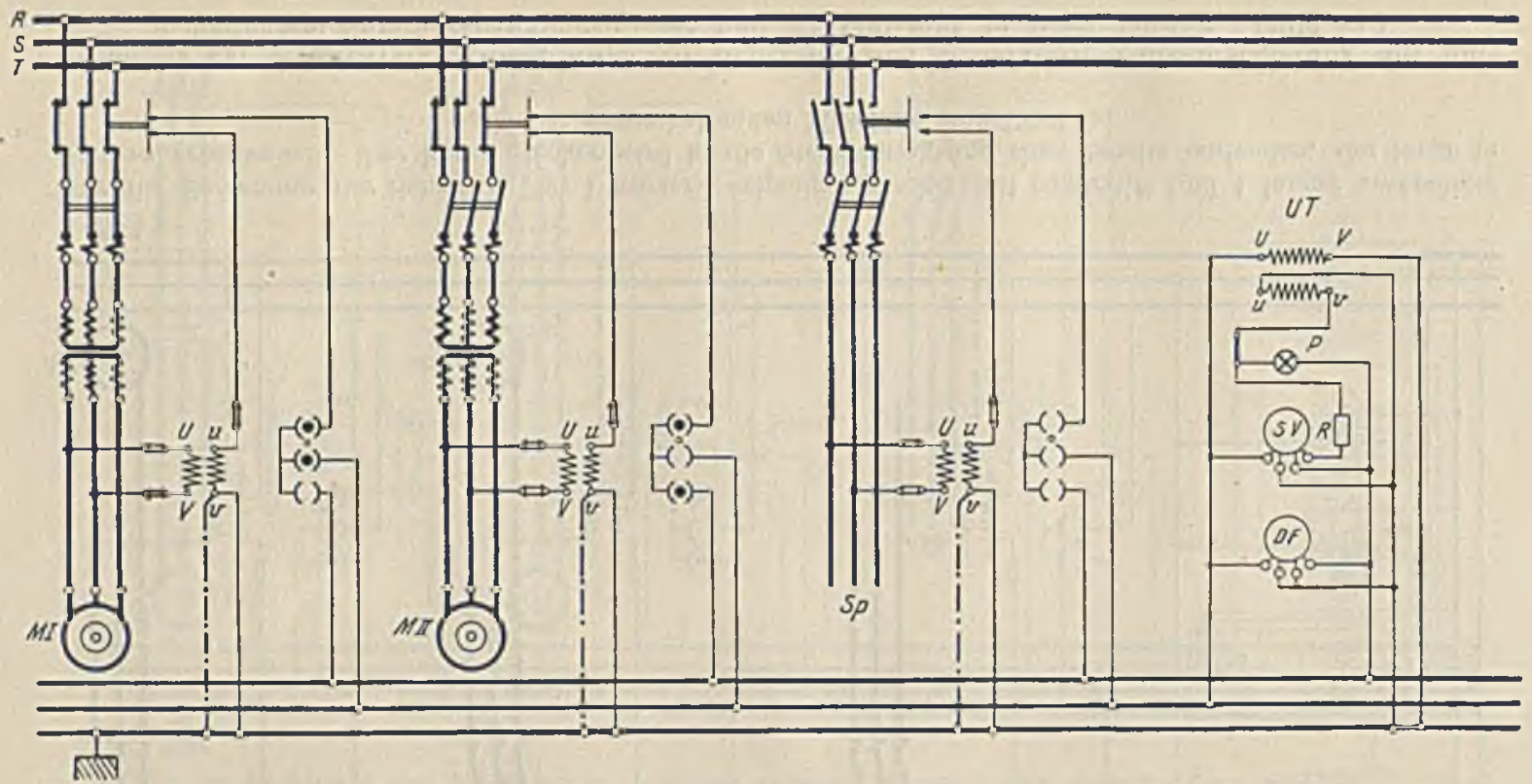

Für die Bedienung der Schaltung ist 1 kurzor zwoipoliger Stecker mit Sperrstift und 1 langer zweipoliger Stecker erforderlich. Der kurzo Stecker wird in die Steckeinrichtung einer bereits laufenden, der lange in die der zuzuschaltenden Maschine eingeführt.

Schaltbild 21. Plasenvergleichung zwischen Generator und Generator. Umkehrschaltung mit Summenspannungsmesser und Thasenlampe in Hellschaltung. (Bild 83.) 


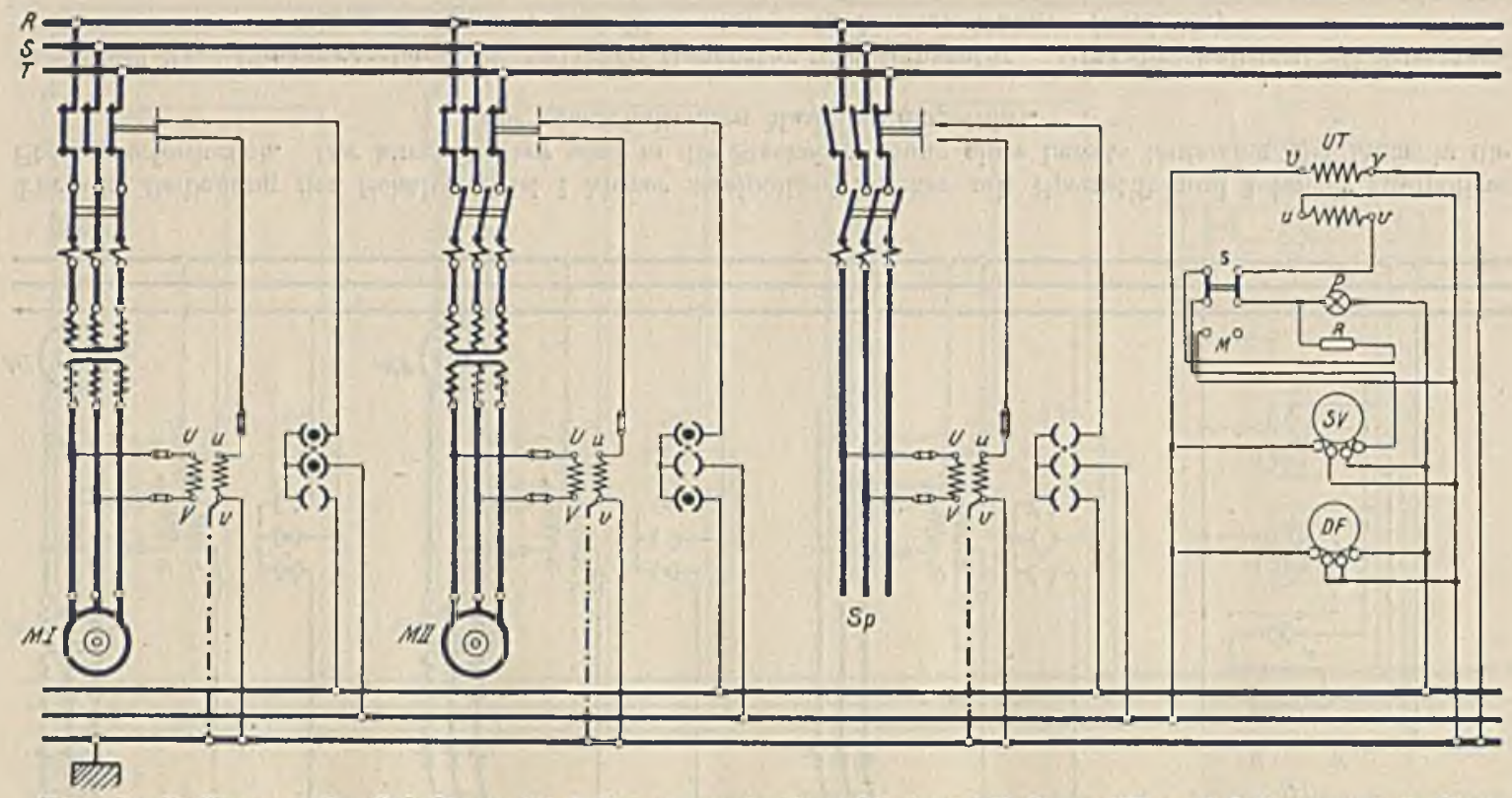

Für die Bedienung der Schaltung ist 1 kurzer zweipoliger Stecker mit Sperrstift und 1 langer zweipoliger Stecker erforderlich. Dor kurzo Stocker wird in dio Steckeinrichtung einer beroits laufenden, der lange in die der zuzuschaltenden Maschine eingeführt.

Schaltbild 22. Phasenvergleichung zwischen Generntor und Generator. Umkehrschaltung mit umschaltbarem Summenspannungsmesser und Phasenlampe in IIellschaltung. (Blld 84.) 


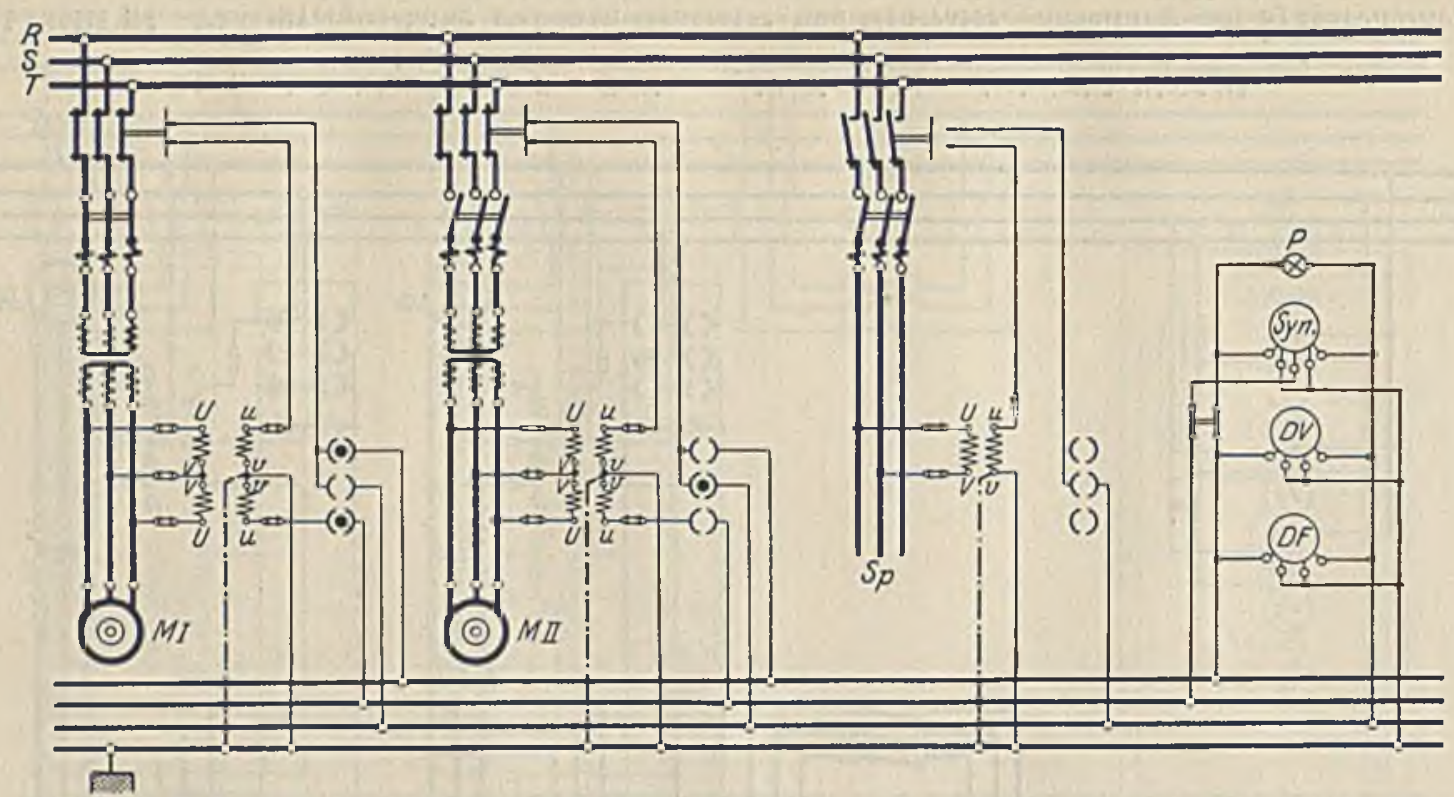

Für die Bedienung der Schaltung ist 1 langer zweipoliger und 1 einpoliger Stecker erforderlich. Der zweipolige Stecker wird in die Steckeinrichtung einer bereits laufenden, der einpolige in die der zuzuschaltenden Maschine eingeführt.

Sehaltbild 23. Phasenvergleichung zwisehen Generator und Generator. Schaltung mit Synchronoskop und Phasenlampe in Dunkelselaltung. (Bild 85.) 


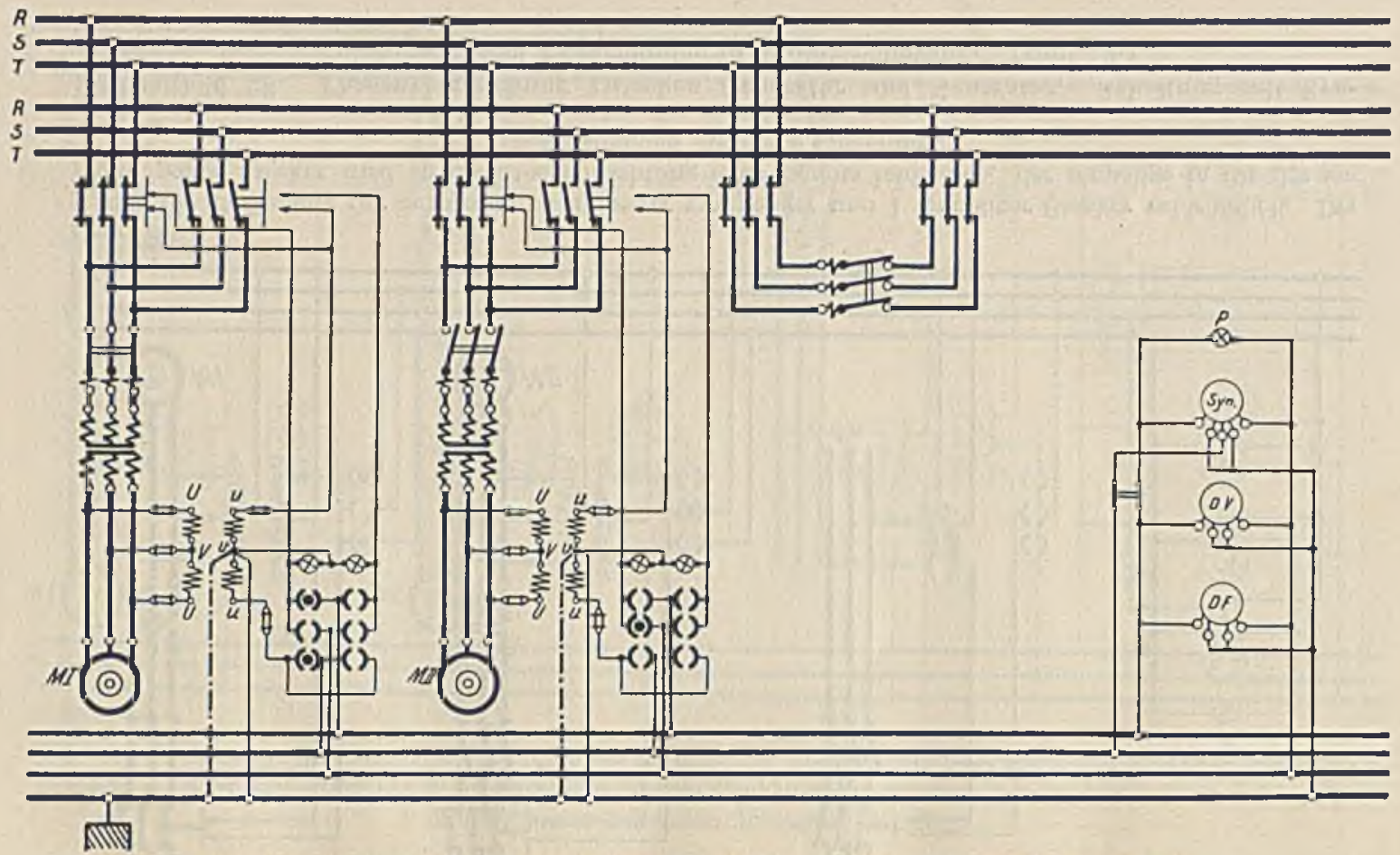

Die Steokeinrichtung und ihre Bedienung ist die gleiche wie bei Schaltbild 23.

Schnltbild 24. Pliasenvergleichming zwischen Generntor und Generator. Schnitung mit Synchronoskop und Phasenlampe in Dunkelschnitung; für Doppelsammelsehienen. (Bild 86.) 


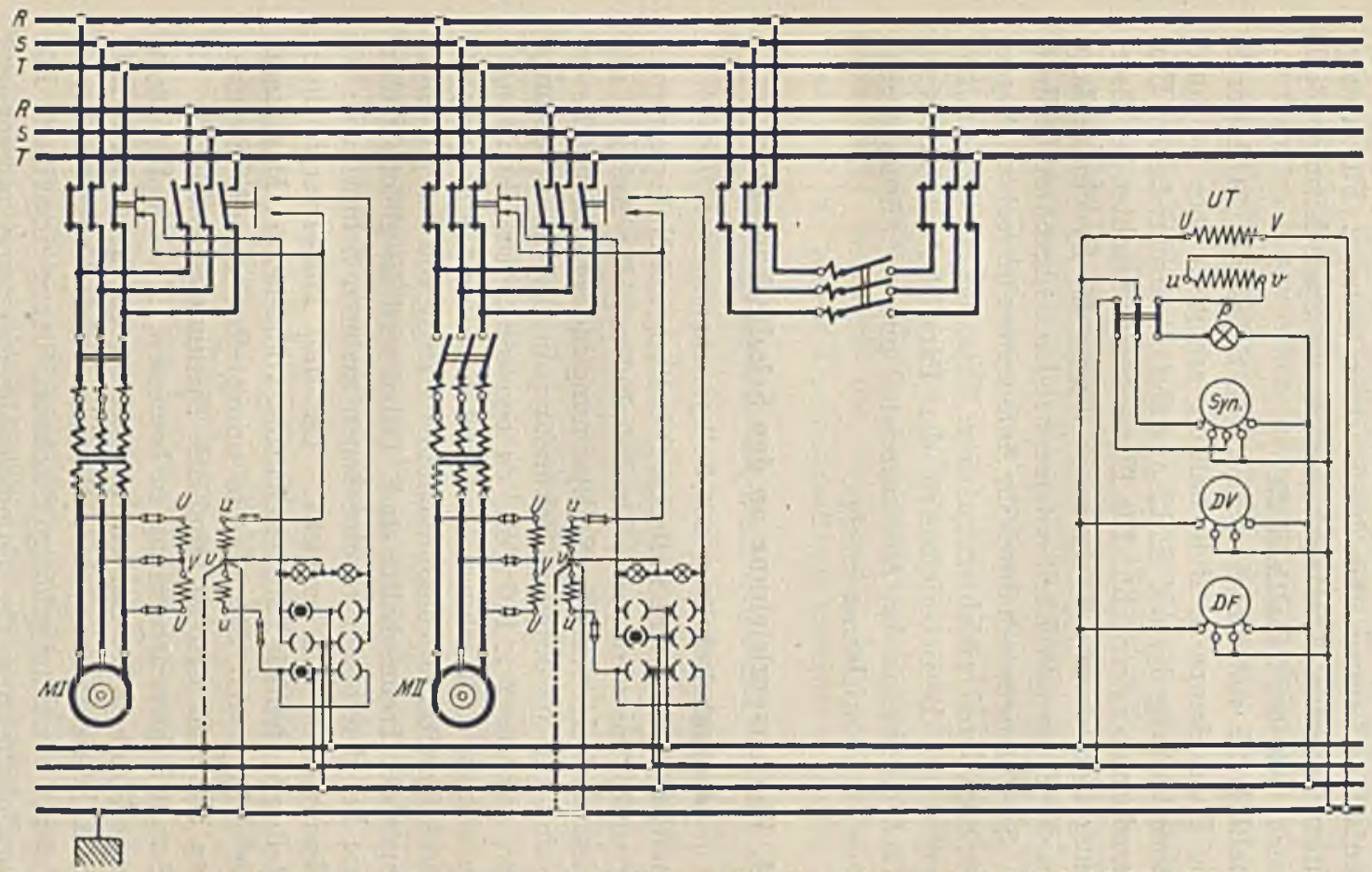

Die Steckeinrichtung und ihre Bedienung ist die gleiche wie bei Schaltbild 23.

Sulıltbild 25. Phnsenyergleichung zwisehen Generator und Generator. Gernischte Schaltung mit Synchronoskop und Phasenlampe in IIellschaltung; fïr Doppelsammelschienen. (Bild 87.) 
poligen Stecker bei einer anderen Maschine, die unverändert weiterlaufen soll. Es müssen dann beim Synchronisieren verschiedenfarbige Lampen über den Steckern brennen. Für etwaige von einem fremden Kraftwerk kommende Speiseleitungen gilt das gleiche wic bei Schaltbild 23 .

Schaltbild 25 unterscheidet sich von Schaltbild 24 dadurch, daß die Phasenlampe in Hellschaltung arbeitet. Dies ist durch Zwischenschaltung des auf S. 12 ausführlich beschriebenen Umkehrtransformators erreicht. Die Phasenlampe leuchtet bei dieser Schaltung stets damn auf, wem der Zeiger des Synchronoskops sich der der Phasengleichheit entsprechenden senkrechten Stellung nähert. Sie gibt demnach durch ihr Aufleuchten im rechten Augenblick ein Signal und erleichtert auf diese Weise das Parallelschalten. Auch wird die Betriebssicherheit der Parallelschalteinrichtung wesentlich erhöht, da das Achtungssignal nur bei störungsfreiem Arbeiten der Einrichtung erfolgt.

\section{Phasenvergleichung an den Schalterkontakten.}

a. Dunkelsehaltung mit Nullspannungsmesser.

Schaltbild 26 zeigt eine indirekte Schaltung mit Nullspannungsmesser und Phasenlampe in Dunkelschaltung für Anlagen mit Doppelsammelschienen. Der Nullspannungsmesser und die Phasenlampe sind für die doppelte Sekundärspannung der Spannungswandler, also für $2 \times 110$ Volt, zu bemessen. Die Schaltungen für die einzelnen Maschinensätze sind bei dicser Schaltung besonders cinfach, da sie vollkommen unabhängig von den jewciligen Stellungen der Trennschalter sind. Dabei wird die gleiche Sicherheit wie bei den bisher beschriebenen anderen Schaltungen für Doppelsammelschienen erreicht. $\mathrm{Da}$ die Phasenvergleichung unmittelbar an dem die Parallelschaltung vollziehenden Maschinenschalter erfolgt und dieser stets unmittelbar an die Sammelschienen angeschlossen ist, sind alle Spannungswandler für die volle Sammelschienenspnnnung zu bemessen. Für die Bedienung der ganzen Anlage ist nur ein zweipoliger Stecker erforderlich. Bei der Inbetriebsetzung einer Maschine wird dieser Stecker in die zu dieser Maschine gehörige Steckvorrichtung eingeführt. Für die Parallelschaltung der Sammelschienensysteme müssen auf beiden Seiten des Kuppelungsschalters besondere Spannungs- 
wandler mit einer besonderen Steckvorrichtung vorgesehen werden. Die Parallelschaltung der Sammelschicnen vollzieht sich dann in der Weise, daß der Stecker in die zum Kuppelungsschalter gehörige Steckvorrichtung eingeführt wird. Die Schaltung etwaiger, von fremden Kraftwerken kommenden Speiseleitungen ist ebenso wie die Schaltung der einz.elnen Maschinensätze auszuführen.

\section{b. Gemischte Schaltung mit Nullspannungsmesser und Umkehr- transformator fïr die Pluasenlampe.}

Sehaltbild 27 unterscheidet sich von 26 nur durch den Finbau des auf S. 12 beschriebenen Umkehrtransformators für die Phasenlampe. Die Phasenlampe liegt demnach hierbei in Hellschaltung und gibt somit durch ihr Aufleuchten das Achtungssignal zum Parallelschalten. Da dies Achtungssignal nur bei vollkommen ordnungsgemäßen Arbeiten der Parallelschalteinrichtung erfolgen kann, wird hierdurch die Betriebssicherheit der Einrichtung gegenüber der reinen Dunkelschaltung wesentlich crhöht.

\section{c. Umkehrsehaltung mit Summenspannungsmesser.}

Schaltbild 28 zeigt eine indirekte Schaltung mit Summenspannungsmesser und Phasenlampe für Hellschaltung. Die Maschinenschaltung ist genau die gleiche wie bei Schaltbild 26. Die Maschinen und Sammelschienen sind demnach gleichpolig, also in Dunkelschaltung auf die Hilfssammelschienen geschaltet. Die Meßeinrichtung dagegen arbeitet unter Verwendung eines Umkehrtransformators in Hellschaltung (vgl. S. 13). Sind kcine besonderen Maschinenspannungsmesser vorhanden, so kann der Summenspannungsmesser in der gleichen Weise wie bei Schaltbild 22 auf $\mathrm{S}$. 92 angegeben umgeschaltet werden.

\section{d. Schaltungen mit Synchronoskop.}

Schaltbild 29 zeigt dic indirekte Schaltung mit SiemensSynchronoskop und Phasenlampe für Dunkelschaltung bei Anlagen mit Doppelsammelschiencn. Das Synchronoskop ist für 110 Volt, die Phasenlampe für $2 \times 110$ Volt zu bemessen. Die Schaltungen für die einzelnen Maschinensätze sind ebenso wie bei den vorhergehenden Schaltbildern 26-28 vollkommen unab. hängig von der jeweiligen Stellung der Trennschalter und daher besonders einfach. Alle Spannungswandler sind bei dieser Schal- 


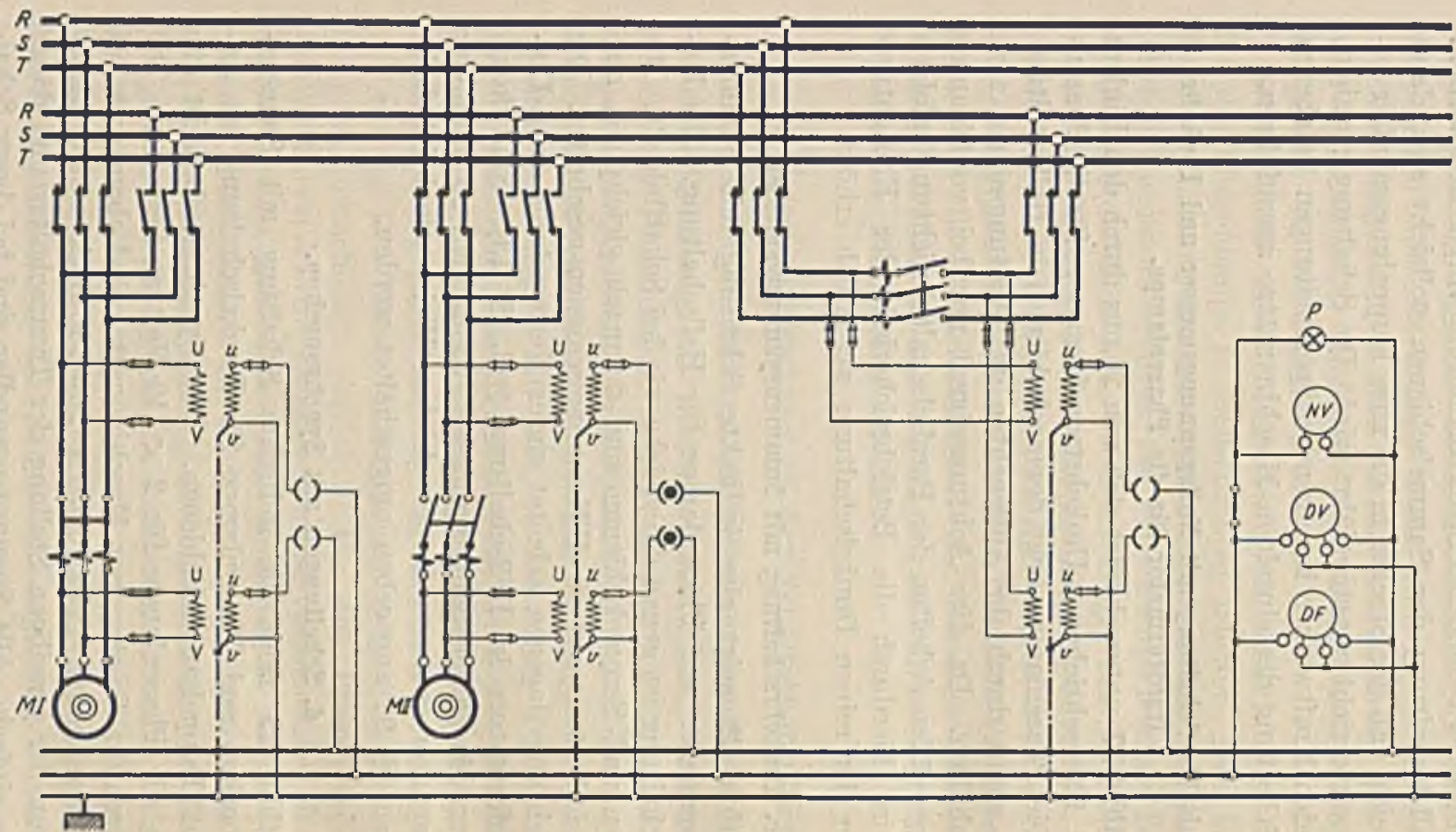

Für die Bedienung der Schaltung ist nur I kurzer zweipoliger Stecker erforderlich, der in die Steckeinrichtung der zuzuschaltenden Maschine eingeführt wird.

Schaltbild 26. Phasentergleichung an den Schalterkontakten. Schaltung mit Nullspannungsmesser und Phasenlampe in Dunkelschaltung; für Doppelsammelschienen. (Bild 88.) 


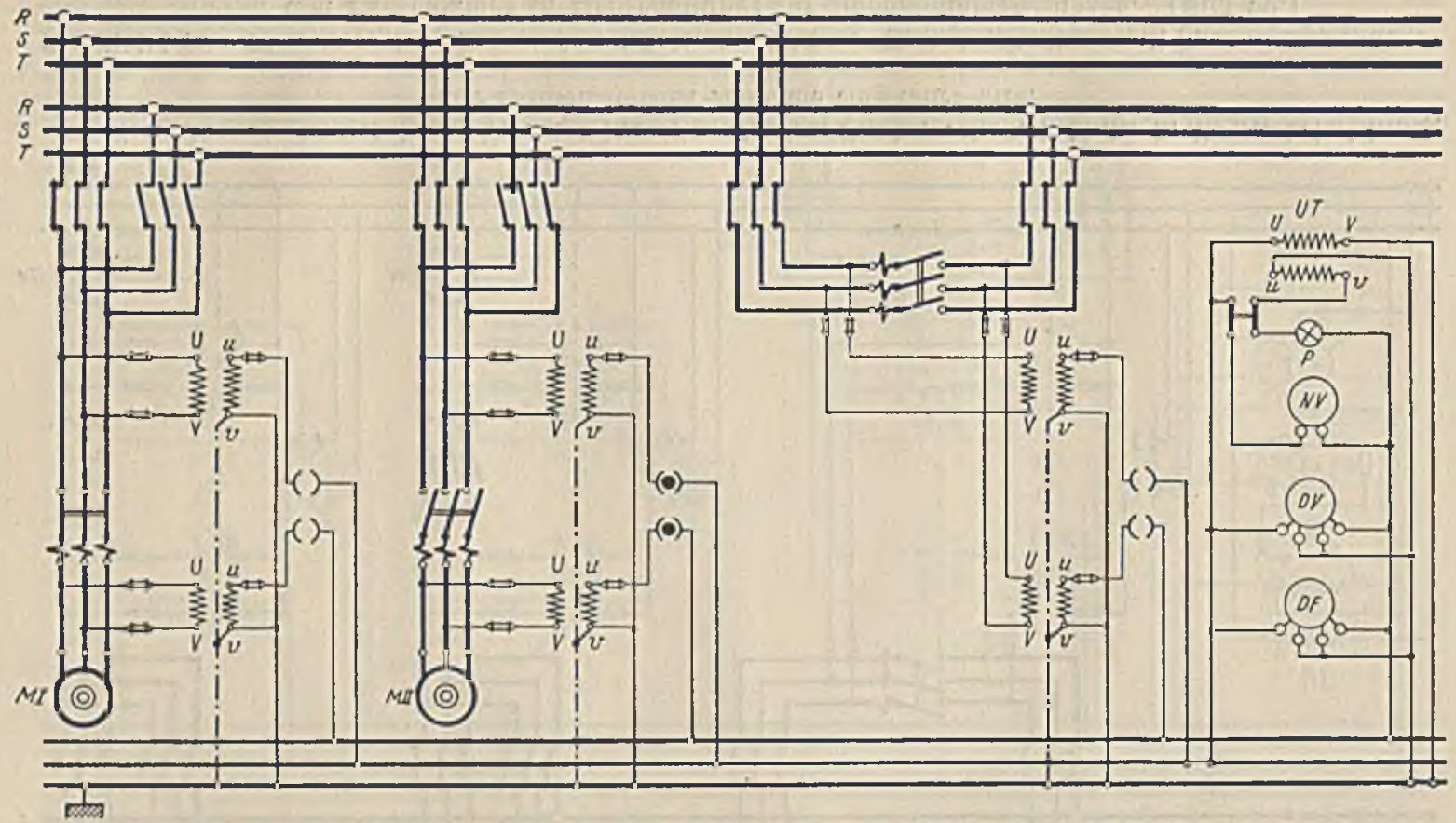

Furir die Bodienung der Schaltung ist nur 1 kurzer zweipoliger Stecker erforderlich, der in die Steckeinriohtung der zuzuschaltenden Maschino eingefübrt wird.

Schaltbild 27. Phasenvergleichung an den Sehalterkontakten. Gemischte Selhaltung mit Nullspannungsmesser und Phasenlampo in Hellschaltung; für Doppelsammelsehiencu. (Bild 89.) 


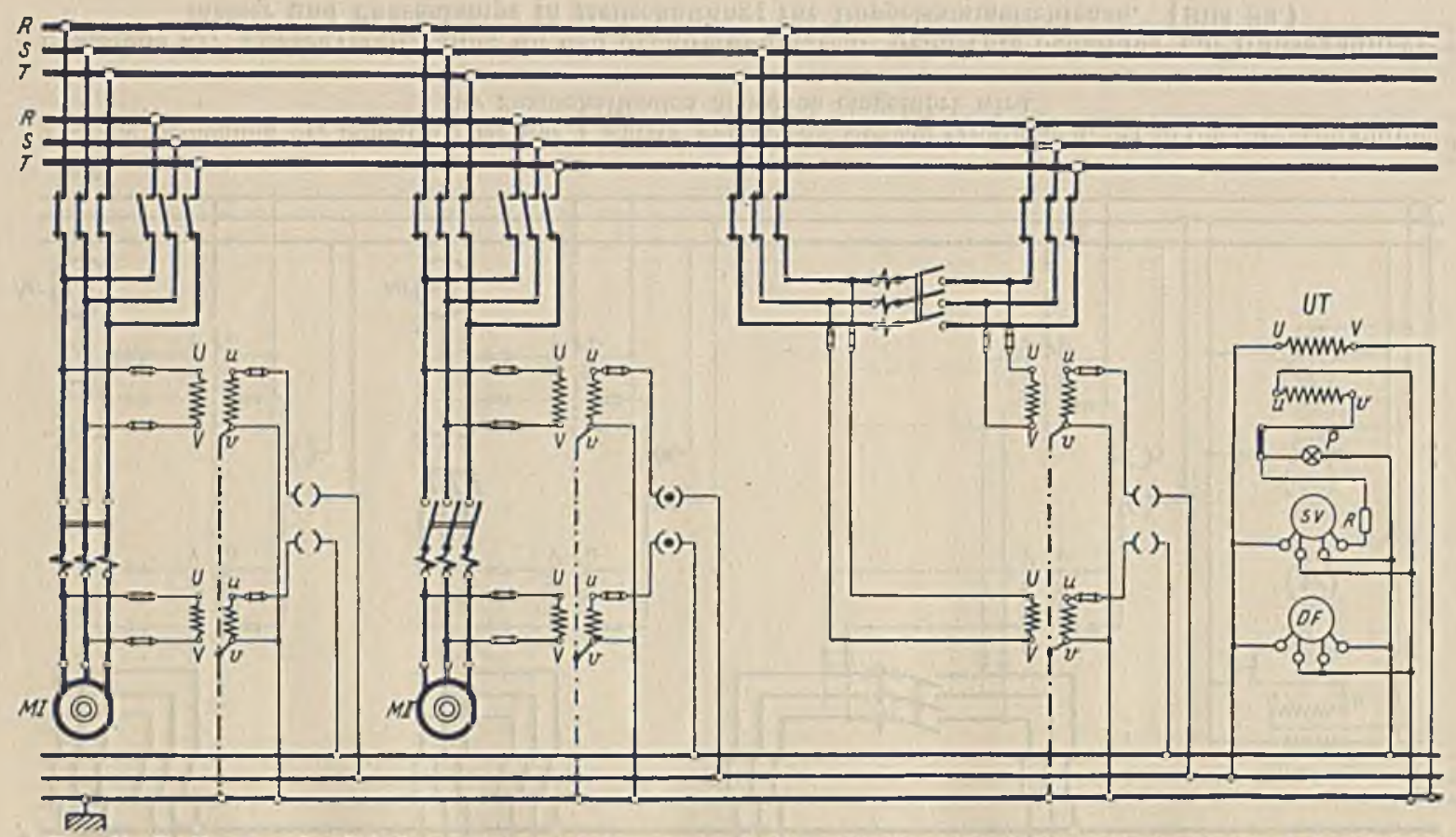

Für die Bedienung der Sohaltung ist nur 1 kurzer zweipoliger Stecker erforderlich, der in die Steckeinrichtung der zuzuschaltenden Masohine eingeführt wird.

Schaitbild 28. Phasenrergleichung an den Schalterkontakten. Umkehrschaltung mit Summenspannungsmesser und Phaseniampe in Hellschaltung; lür Doppelsammelschienen. (Bild 90.) 


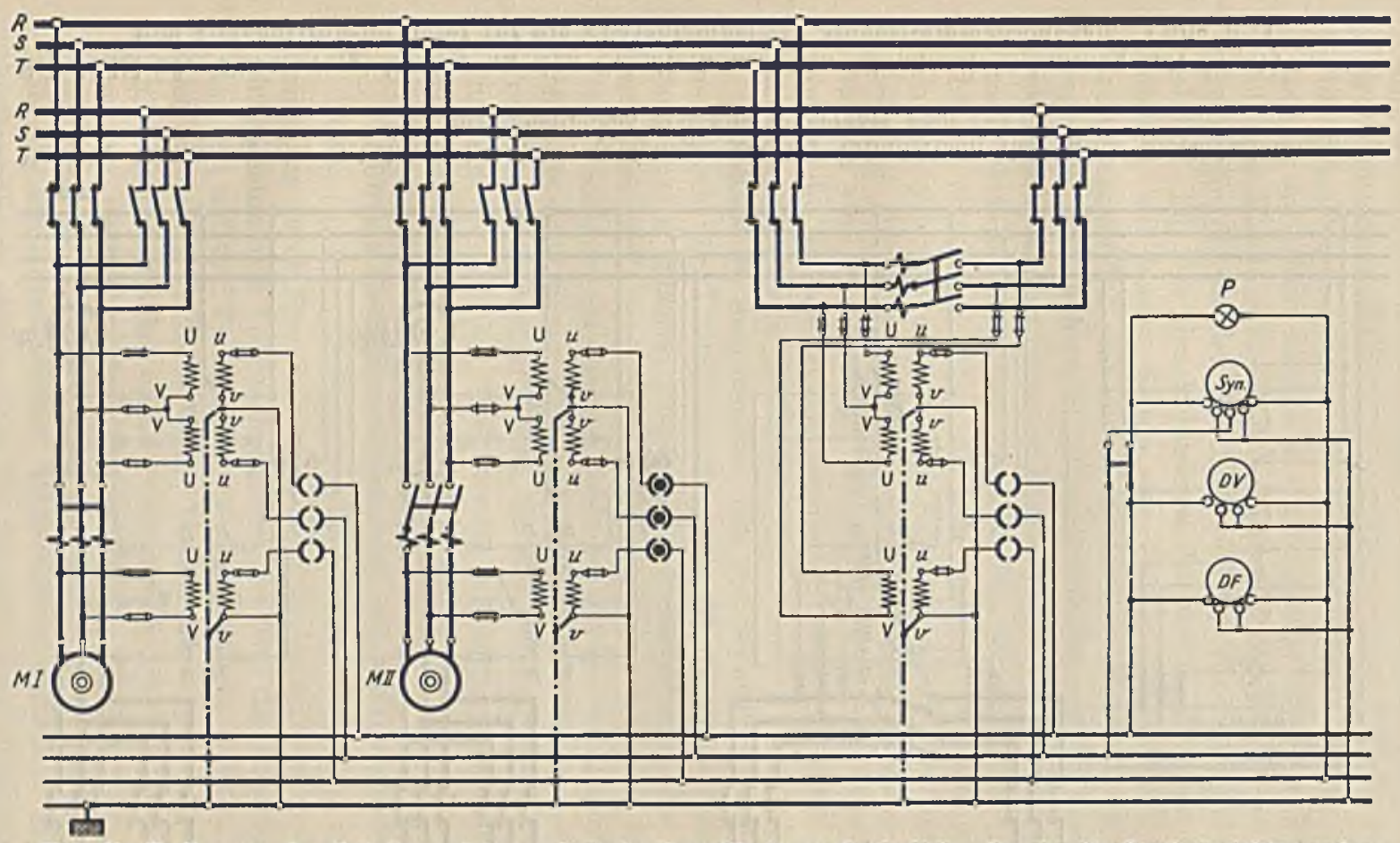

Für die Bedienung der Schaltung ist nur 1 dreipoliger Stecker erforderlich, der in die Steckeinrichtung der zuzuschaltenden Maschine eingoführt wird.

Schaltbild 29. Phasenvergleichung an den Schalterkontakten. Schaltung mit Synchronoskop und Phasenlampo in Dankelschaltung; Pür Doppelsammelschienen. (Bild 91.) 


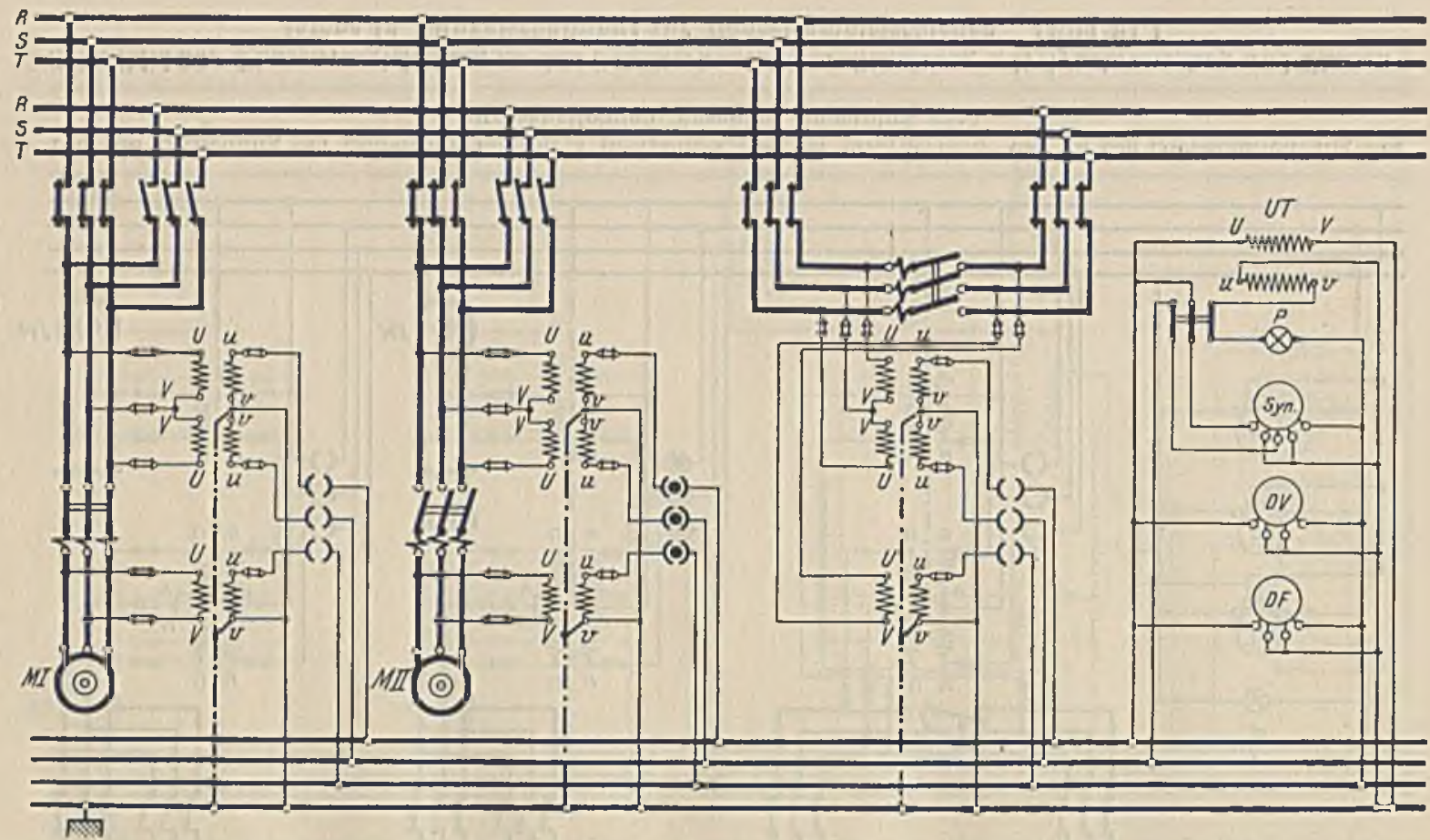

Für die Bodienung der Schaltung ist nur 1 dreipoliger Stecker erforderlich, der in die Steckeinrichtung der zuzuschaltenden Maschino eingefülırt wird.

Schaltbild 30. Phasenvergleichung an den Schalterkontakten. Gemischte Schaltung mit Synchrouoskop und Umkehrtranslormator für die Phasenlampe; für Doppelsammelschienon. (Bild 92.) 
tung ebenfalls für die volle Sammelschienenspannung zu bemessen, da die Naschinenschalter stets unmittelbar, ohne Zwischenschaltung von Transformatoren, an den Sammelschienen liegen. Zur Bcdienung der ganzen Anlage ist ein dreipoliger Stecker erforderlich. Soll eine Maschine in Betrieb genommen werden, so wird dieser Stecker in die zu der Maschine gehörige Steckvorrichtung eingeführt. Für das Synchronisieren der Sammelschienen sind besondere, auf beiden Seiten des Kuppelungsschalters liegende Spannungswandler mit einer zugehörigen Steckvorrichtung erforderlich. Die Drehstrom-Spannungswandler schließt man hierbei zweckmäßig an das Sammelschienensystem an, an dem betriebsmäßig stets mehrere Maschinen laufen, während man den Einphasen-Spannungswandler an das weniger belastete Sammelschienensystem anschließt. Nan regelt dann beim Synchronisieren stets dic an dem weniger belasteten Sammelschienensystem laufende einzelne Maschine. Etwaige von fremden Kraftwerken kommende Speiseleitungen werden in gleicher Weise wie die cinzelnen Maschinen geschaltet.

Schaltbild 30 unterscheidet sich von Schaltbild 29 nur durch den eingebauten Umkchrtransformator für die Phasenlampe. Dic Phasenlampe arbeitet demgemäß in Hellschaltung. Sic leuchtet stets dann auf, wenn der Zeiger des Synchronoskops durch die dem Synchronismus entsprechende senkrechte Stellung hindurchgeht. Es wird demgemäß auch hier die Betriebssicherheit der Vorrichtung wesentlich erhöht, da das Achtungssignal durch die Phasenlampe nur bei vollkommen ordnungsgemäßen Arbeiten der Vorrichtung erfolgen kann.

\section{e. Direkte Hochspannungsschaltung mit MeBkondensatoren.}

Arbeiten zwei Kraftwerke, deren Sammelschienen nicht unmittelbar durch Speiseleitungen miteinander verbunden sind, auf cin gemeinsames Versorgungsgcbiet, so $\mathrm{muB}$ die Parallschaltung draußen auf der Fernleitungsstrecke an einem Punkte, an dem sich die beiden Verteilungsnetze berühren, erfolgen. Da die Netze an diesen Schaltstellen im allgemeinen dauernd verbunden bleiben, und nur nach etwaigen Betricbsstörungen von neuem parallelgeschaltet werden, wird man für diese Schaltstellen nicht die teueren Einrichtungen mit Meßwandlern einbauen, zumal die MeBwandler in diesem Falle für die hohe Fornleitungsspannung, 
104
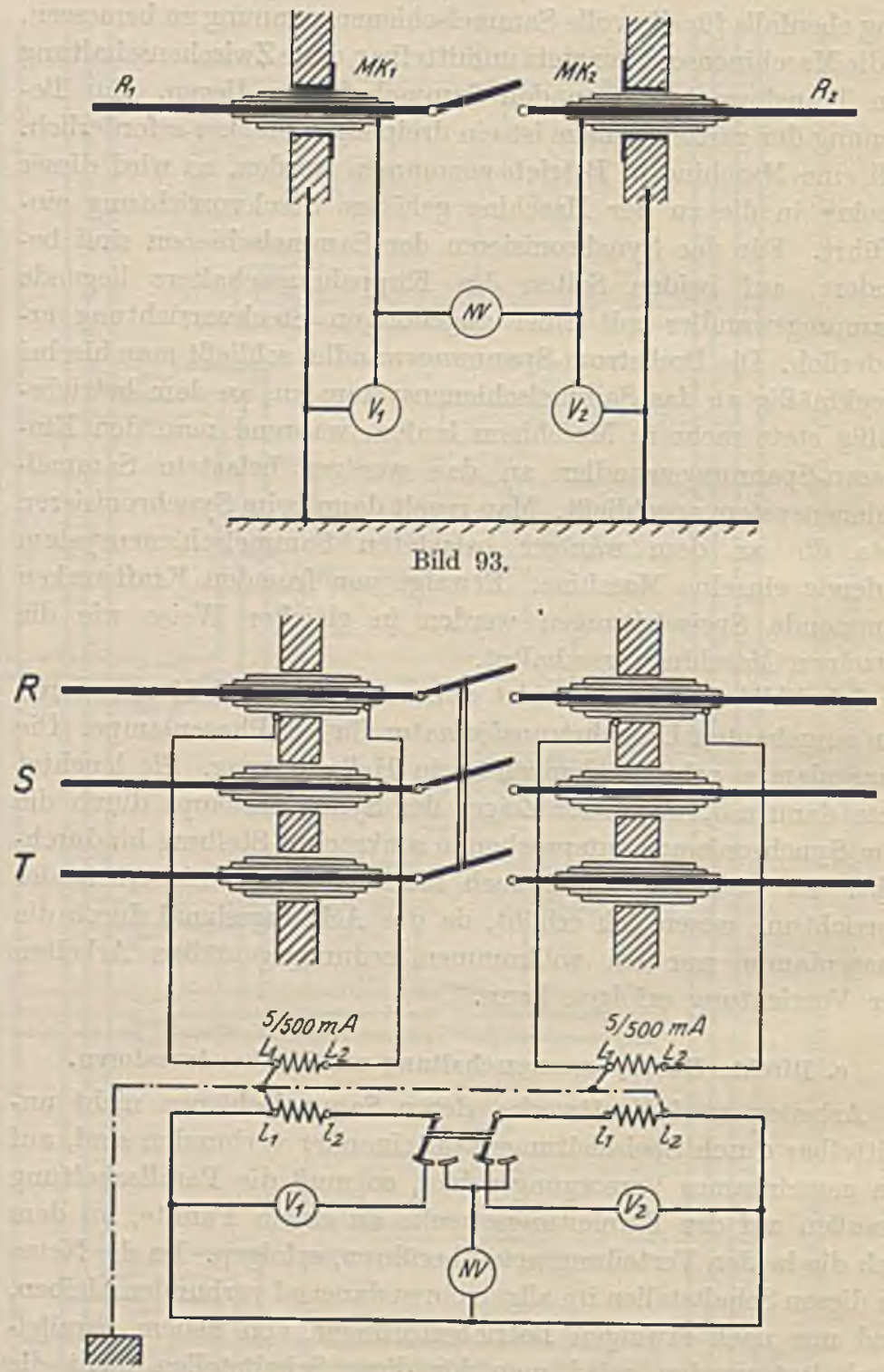

Bild 94.

Tafel 20. Hochspannuugs-Schaltungen mit Meßkondensatoren. 
beispielsweise für 100000 Volt, ausgeführt werden müßten. In Amerika werden in diesen Fällen elektrostatische Spannungsmesser, die an besondere Meßkondensatoren angeschlossen sind, verwendet. Die Instrumente sind hierbei nur für eine verhältnismäBig kleine, an den Meßkondensatoren abgegriffene Teilspannung bomessen. Den Siemens-Schuckert-Werken ist eine Schaltweise gesetzlich geschützt, bei der sich besondere Meßkondensatoren dadurch erübrigen, daß die als Durchführungskondensatoren ausgebildeten Durchführungsisolatoren des die Parallelschaltung vollzichenden Hochspannungsschalters als Meßkondensatoren benutzt werden. Die Schaltung wird hierbei aus Billigkeitsgründen meist nur cinpolig ausgeführt, wie es das nebenstehende Prinzipschaltbild zeigt. In diesem sind $R_{1}$ und $R_{2}$ dic $z u$ verbindenden Hochspannungsleitungen und $M K_{1}$ und $M K_{2}$ die als Meßkondensatoren benutzten Durchführungskondensatoren des Hochspannungsschalters. Durch die Befestigungsstellen der Meßkondensatoren an der Wand des Schalthauses ist das Erdpotential der Meßschaltung gegeben, während durch zwci symmetrisch gelegene Zwischenbelege zwei Vergleichspotentiale geschaffen werden. Liegen diese auf gleicher Höhe, so zeigt dor an sie angeschlosseno Nullspannungsmesser die Spannung Null an. Es kann also parallelgeschaltet werden. Die Spannungen der beiden Zwischenbelege gegen Erde geben ein Maß für die auf beiden Sciten des Schalters bestehenden Netzspannungen. Sie werden durch die Spannungsmesser $V_{1}$ und $V_{2}$ gemessen.

Um die mechanisch sehr empfindlichen elektrostatischen Spannungsmesser ganz zu vermeiden, verwendet S. \& H. neuerdings eine andere Schaltung. Bei dieser werden besondere Stromwandler benutzt, die von dem Kondensatorstrom durchflossen werden. Durch diese Stromwandler wird der sehr kleine Kondensatorstrom auf eine größere, meßbare Stromstärke hinauftransformiert, so daß man an Stelle der elektrostatischen Spannungsmesser Dreheisen-Instrumente benutzen kann. Da die Stromwandler sekundär stets geschlossen sein müssen, wird hierbei ein besonderer Umschalter ohne Stromunterbrechung benutzt. In der linken Schaltstellung werden die beiden Netzspannungen durch die Spannungsmesser $V_{1}$ und $V_{2}$ gemessen, während in der rechten Stellung des Schalters der Nullspannungsmesser angeschlossen ist. Das Parallelschalten mittels dieser Vorrichtung 
vollzieht sich anstandslos. Allerdings muß man, da man hierbei keine Regelvorrichtung für die parallelzuschaltenden Netze zur Verfügung hat, warten, bis ein günstiger Augenblick zufälligerweise eintritt. Dies kann unter Umständen stundenlang dauern, jedoch ist dieser Nachteil insofern nicht schwerwiegend, als die Parallelschaltung, wie bereits anfangs erwähnt, nur selten ausgeführt werden muß.

\section{Besondere Maßnahmen bei verschiedenartig geschalteten Haupttransformatoren.}

Solange die Maschinen eines Kraftwerkes unter sich parallel geschaltet werden sollen, entstehen durch die zwischen den Generatoren und Sammelschienen liegenden Haupttransformatoren für die Parallelschaltung keine Schwierigkeiten, da man in diesem Falle fast stets verlangen kann, daß die Haupttransformatoren aller Maschinen gleichartig geschaltet sind. Sollen jedoch verschiedene Kraftwerke untereinander parallel geschaltet werden, wic dies jetzt bei der Zusammenschließung der Kraftwerke zu Landesversorgungsnetzen erforderlich ist, so kann man nicht mehr voraussetzen, daß die Haupttransformatoren der von verschiedenen Firmen gebauten Kraftwerke alle gleich geschaltet sind. Man muß daher in diesem Falle bei den Parallelschaltvorrichtungen besondere Einrichtungen vorsehen, durch welche die durch die Schaltung der Haupttransformatoren verursachte Phasenverschiebung kompensiert wird. Um an Kosten zu sparen, wird man diese Einrichtungen stets so wählen, daß man in der von einem fremden Kraftwerk kommenden Hochspannungs-Speiseleitung nur einen einphasigen Spannungswandler braucht. Unter dieser Voraussctzung sind in den folgenden Abschnitten die für die verschiedenen Schaltarten der Haupttransformatoren von den SiemensSchuckert-Werken angewandten Hilfsschaltungen entwickelt.

Schaltart A. Bei der auf Tafel 21 dargestellten Schaltart A ist die Primärwickelung und die Sekundärwickelung der Haupttransformatoren vollkommen gleichartig geschaltet. Es sind sowohl prinı̈r als sekundär die Anfänge der drei Wickelungen zum Sternpunkt verbunden, so daß die Vektordiagramme für die Primärwickelung und die Sekundürwickelung vollkommen gleich sind. Die für die Parallelschaltung benutzten verketteten Spannungen $U V$ und $u v$ sind daher in Phase. Aus diesem Grunde ist auch 
für die Parallelschaltung keine besondere Maßnahme erforderlich, d. h. dic Maschinen können ohne weiteres mit einer von einem fremden Kraftwerk kommenden Hochspannungs-Speiseleitung parallel geschaltet werden.

Schaltart B. Bei der auf Tafel 22 dargestellten Schaltart B ist auch primär und sekundär Sternschaltung angewandt, jedoch sind auf der Unterspannungsseite die Enden und auf der Oberspannungsseito die Anfänge der drei Wickelungen zum Sternpunkt vereinigt. Hieraus ergeben sich für die Unterspannungsscite und die Oberspannungsseite entgegengesetzte Richtungen der Einzelspannungen und damit auch der verketteten Spannungen. Dic Oberspannung wirkt in der Richtung $V U$, während die Unterspamnung um $180^{\circ}$ verschoben ist und in der Richtung $u v$ wirkt. Bei der Ausführung der Schaltung ist die dieser Phasenverschiebung entsprechende Vertauschung der beiden Leitungen infolge der vorhandenen Erdungsleitungen der Meßwandler nicht ohne weiteres möglich. Man schaltet daher einen im Verhältnis $1: 1$ übersetzenden Isoliertransformator dazwischen und vertauscht die Sekundäranschlüsse, wic es in Bild 104 gezeigt ist.

Schaltart C. Bei der auf Tafel 23 dargestellten Schaltart C ist die Oberspannungsseite der Haupttransformatoren im Dreieck geschaltet, während die Unterspannungsseite in Sternschaltung liegt. Auf der Unterspannungsseite sind die Enden der drei Wickelungen $u_{2}, v_{2}, w_{2}$ zum Sternpunkt verbunden. Die Einzclspannungen sind daher, wie das Diagramm zeigt, nach dem Sternpunkt hin gerichtet. Die verkettete Unterspanuung wirkt im Sinne $u v$, während die entsprechende Oberspannung im Sinne $V U$ wirkt. Außerdem sind die beiden Spannungen noch räumlich um eimen Winkel von $30^{\circ}$ gegeneinander verschoben. Man kann diesen Winkel mit Hilfe der anderen Drehstromspannung wu ausgleichen, indem man diese so an den Endpunkt $u$ des Diagramms ansetzt, daß ein gleichschenkeliges Dreieck $v w^{\prime} u^{\prime}$ entsteht. Die Resultierende $u^{\prime} v$ liegt dann parallel zur Oberspannung $\nabla U$, wirkt jedoch in entgegengesetzter Richtung. Die diesem Diagramm entsprechende Hilfsschaltung ist in Bild 109 dargestellt. Es sind hierbei zwei Hilfstransformatoren benutzt, dic im Verhältnis $110: 63,5$ übersetzen. Der für beide Hilfstransformatoren gemeinsame Punkt $u$ ist in die Mitte verlegt, so daß der linke Hilfstransformator der Spannung vu und der rechte Hilfstransformator der 

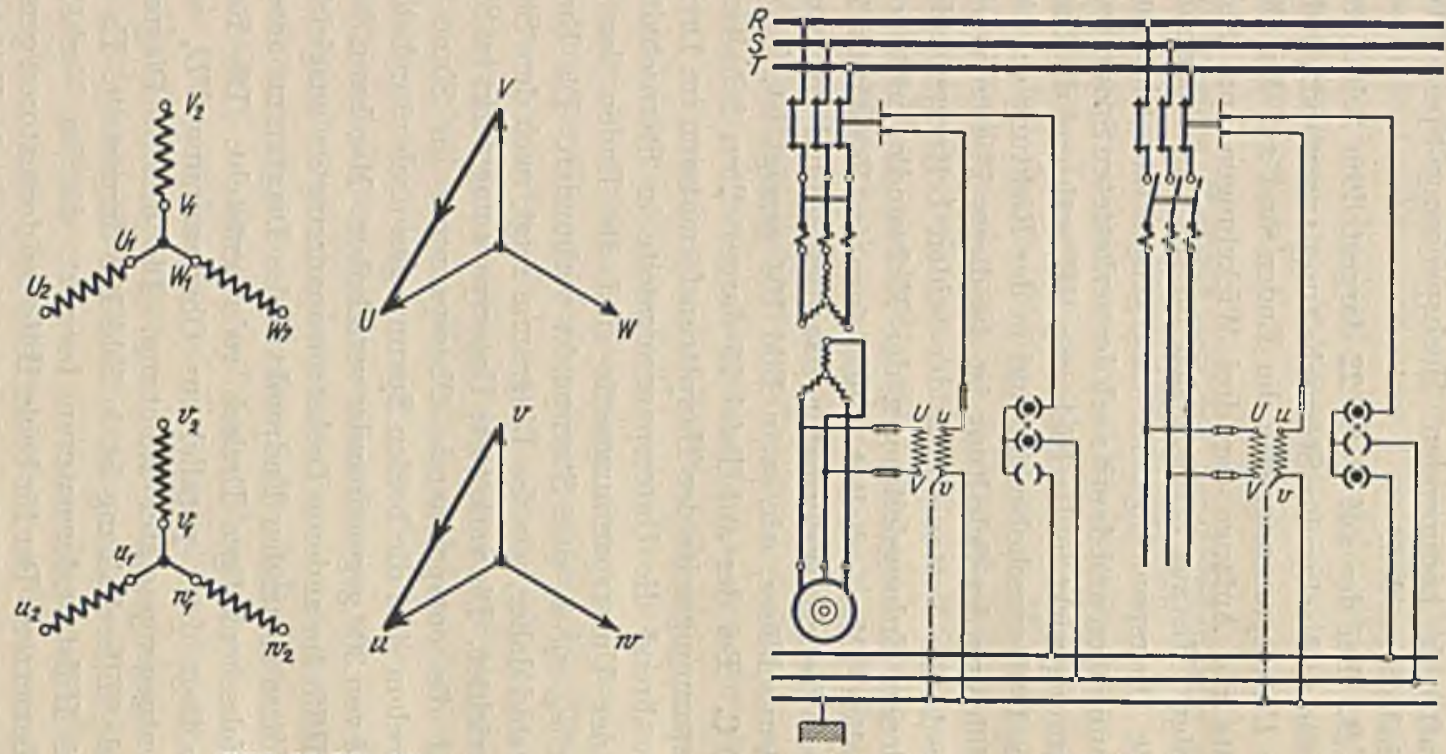

Bild 95 bis 98 .

Bild 99.

Tafel 21. Schaltung der Haupttransformatoren nach Schaltart A. 


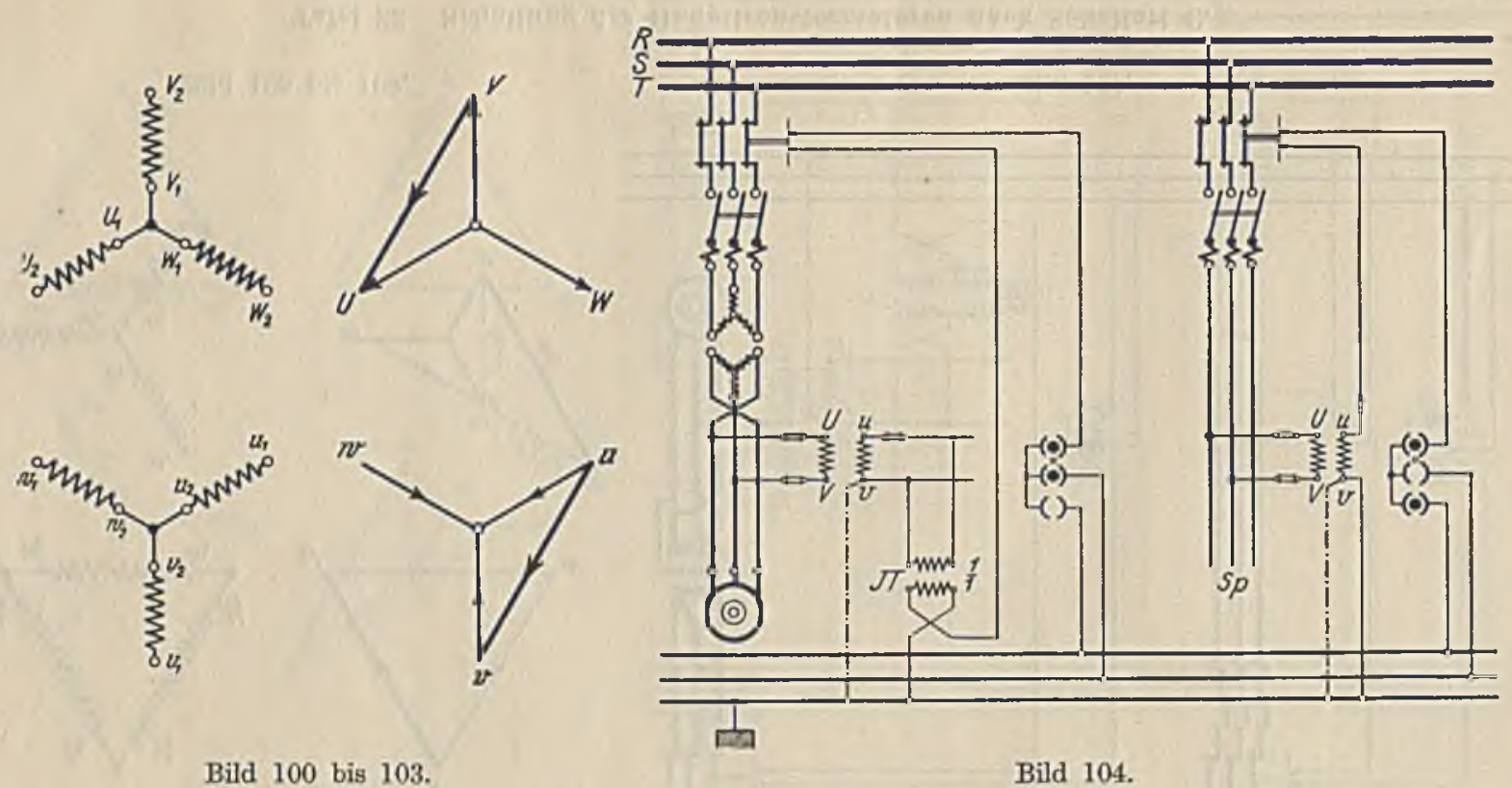

Tafel 22. Schaltung der Inapttransformatoren nach Schaltart B. 

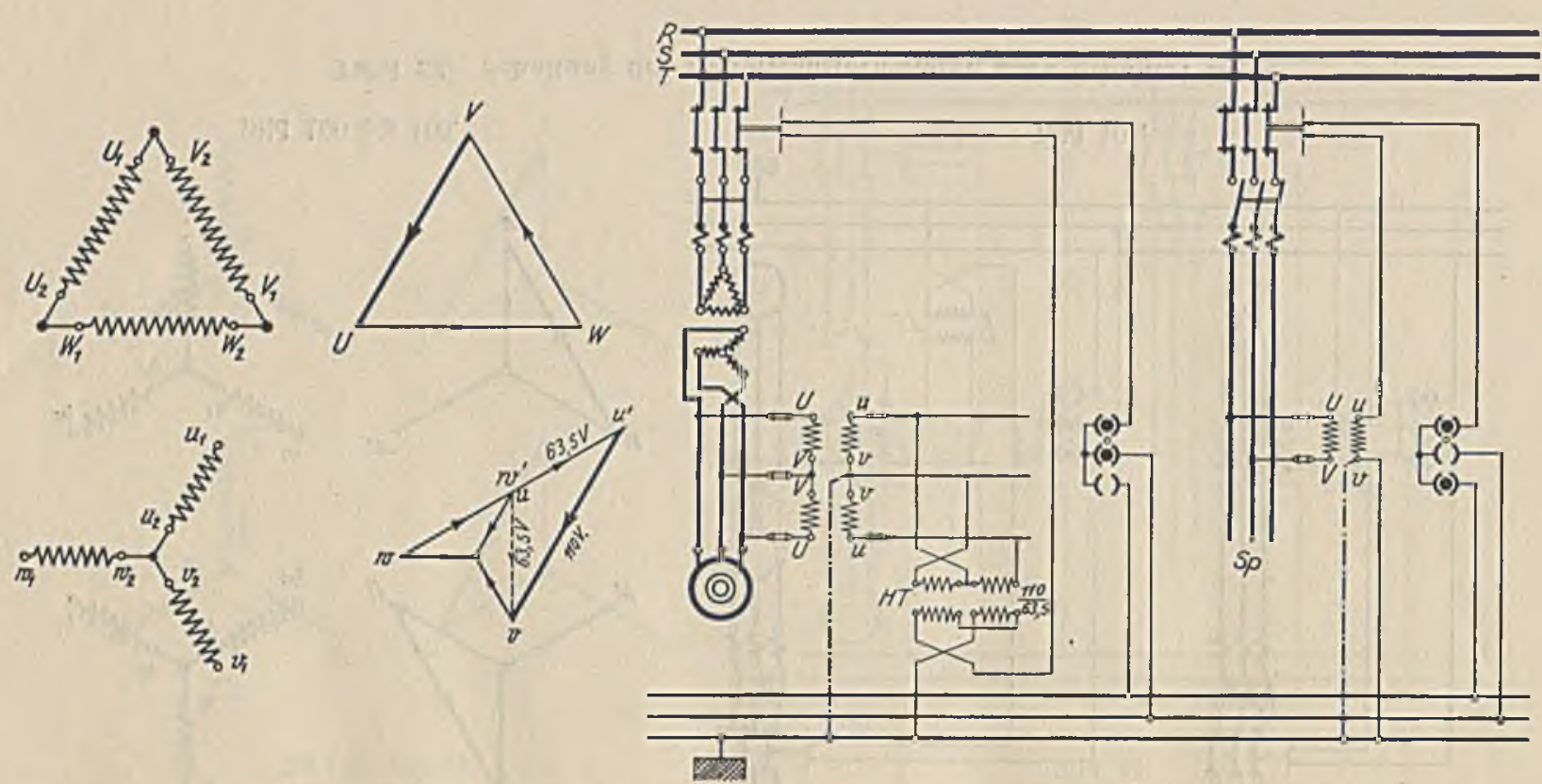

Bild 105 bis 108 .

Bild 109.

Tafel 23. Schaltung der Haupttransformatoren nach Schaltart c. 

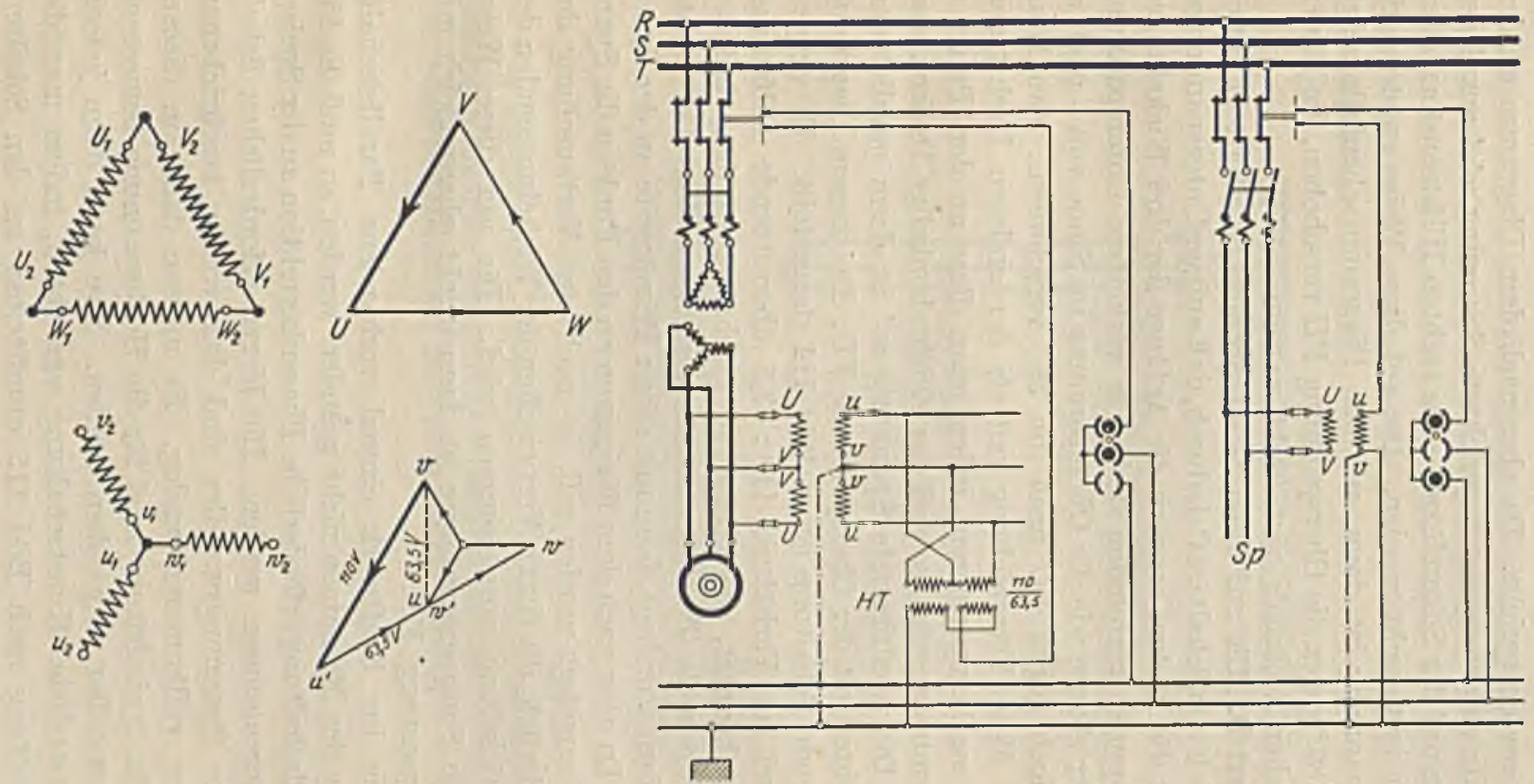

Bild 110 bis 113.

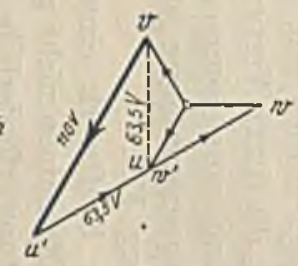

Bild 114.

Tafel 24. Schaltung der Haupttransformatoren nach Schaltart D. 
Spannung $u w$ entspricht. Da aber nach dem Diagramm an den Punkt $u$ die entgegengesetzt gerichtete Spannung $w^{\prime} u^{\prime}$ anzulegen ist, müssen auf der Sekundärseite des rechten Hilfstransformators die Enden vertauscht werden. Die auf dicse Weise entstehende Summenspannung ist dann, wie das Diagramm ebenfalls zeigt, noch um $180^{\circ}$ gegen die Oberspannung $V U$ verschoben, die Enden müssen daher nochmals vertauscht werden.

Schaltart D. Die auf Tafel 24 dargestellte Schaltart D unterscheidet sich von Schaltart $C$ dadurch, da $B$ auf der Unterspannungsseite nicht die Enden, sondern die Anfänge der drei Wickelungen zum Sternpunkt verbunden sind. Die verkettete Unterspannung wirkt daher ebenso wie die Oberspamnung im Sinne von $v$ nach $u$, sie ist jedoch gegen $V U$ noch um $30^{\circ}$ verschoben. Man kann auch hier diese Verschiebung mittels der anderen Drehstromspannung uw kompensieren, indem man diese an den Punkt $u$ des Diagramms ansetzt, so daß das gleichschenkelige Dreieck $v u u^{\prime}$ entsteht. Die resultierende Spannung $v u^{\prime}$ ist dann parallel und gleichgerichtet mit der Oberspanung $\nabla U$. Die diesem Diagramm entsprechende Schaltung ist in Bild 114 dargestellt. Es werden wieder zwei im Verhältnis $110: 63,5$ übersetzende Hilfstransformatoren benutzt, die primär so geschaltet werden, daß der Punkt $u$ für beide Transformatoren gemeinsam ist. Der rechtc Hilfstransformator liegt dann mit seiner Primärseite an der Spannung $u w$. Da aber nach dem Diagramm an den Punkt $u$ die Spannung $w^{\prime} u^{\prime}$ angelegt werden soll, ist noch eine Vertauschung der Pole erforderlich, die durch Vertauschen der Wickelungsenden der Sekundärwickelung vorgenommen wird. Die auf diese Weise entstehende Summenspannung ist dann direkt phasengleich mit der Oberspannung $V U$.

Soll die im Kraftwerk einmal vorhandene Parallelschalteinrichtung der Maschinen nicht geändert werden, so muß die für die Parallelschaltung erforderliche Phasenkorrektion an der Speiseleitung vorgenommen werden. Die hierzu erforderlichen Schaltungen der Spannungswandler sind den vorher beschriebenen Schaltungen vollkommen analog. Es müssen daher in diesem Falle für die Speiseleitung zwei für die Hochspannung bemessene Spannungswandler vorgesehen werden. Man kann dann jedoch auf die zusätzliche Kunstschaltung verzichten, indem man die Parallelschaltung nach Bild 115 unmittelbar an den Schalter- 
liontakten vornimmt. Die Steckeinrichtungen der Speiseleitung können hierbei, wie es im Schaltbild gezeigt ist, genau so ausgebildet sein, wie die der einzelnen Maschinen, so da $B$ das Parallelschalten genau in der gleichen Weise wie bei den einzelnen Maschinen vorgenommen wird.

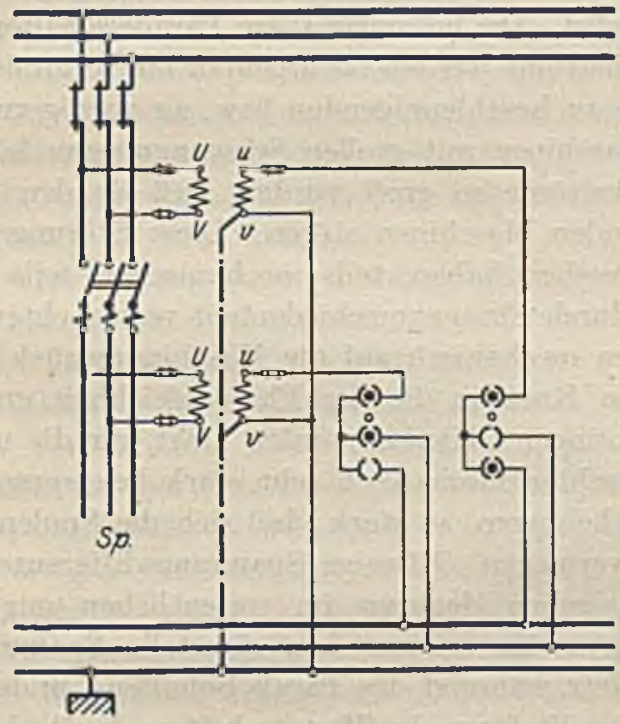

Bild 115.

Der an die Hilfssammelschienen anzuschließende Instrumentsatz kann bei allen vorstehend beschriebenen Schaltungen beliebig nach den Schaltbildern 18-22 gewählt werden. 


\section{Einrichtungen zum selbsttätigen Parallelschalten.}

\section{a. Anwendungsgebiete.}

Je größer die parallel zu schaltenden Maschinen sind, mit um so größerer Sorgfalt muß das Parallelschalten erfolgen, da mit der Leistung der Maschineneinheiten auch die Energie der. Ausgleichströme anwächst. Die bei ungenauem Parallelschalten entstehenden Ausgleichströme werden naturgemäß um so größer, je größer die durch sie zu beschleunigenden bzw. zu verzögernden Massen sind. Bei Maschinen mit großen Schwungmassen können daher die Ausgleichströme so groß werden, daß sie den Betrieb der bereits laufenden Maschinen stören. Diese Störungen sind, wie wir früher gesehen haben, teils mechanischer, teils elektrischer Natur. Die durch Phasenverschiedenheit verursachten Ausgleichströme wirken mechanisch auf die Maschine zurück und suchen sie mit einem Ruck in die der Phasengleichheit entsprechende Stellung zu bringen. Hierbei werden nicht nur die umlaufenden Teile der Maschine mechanisch sehr stark beansprucht, sondern auch die Wickelungen, so stark, daß sich die Spulenköpfe unter Umständen verbiegen. Etwaige Spannungsdifferenzen beim $\mathrm{Pa}$. rallelschalten geben dagegen im wesentlichen nur elektrische Rückwirkungen. Sie verursachen einesteils Spannungsschwankungen im Netz während des Parallclschaltens, andernteils aber entstehen beim Einlegen des Hauptschalters durch das plötzliche Kurzschließen der Differenzspannungen zwischen Netz und zuzuschaltender Maschine häufig elektrische Sprungwellen, die die Isolation der ersten Spulen der. Maschinenwickelungen beanspruchen. Alle diese Störungen können nur bei ganz sorgfältigem Parallelschalten vermieden werden. Die Anforderungen, die hierdurch an den Schalttafelwärter gestellt werden, werden noch größer, wenn noch die weitere Forderung hinzutritt, daß etwaige Ausgleichströme nicht dem Netz entnommen, sondern von der zuzuschaltenden Maschine ins Netz hineingeliefert werden. Diese Forderung ist insofern berechtigt, als in den meisten Fällen das Netz schon stark belastet ist, che eine weitere Maschine in Betrieb genommen wird. Ein derartig stark belastetes Netz ivürde aber die zusätzliche Stoßbelastung einer schlecht parallelgeschalteten, als Motor laufenden Maschine nicht mehr aushalten. Um es zu erreichen, daß die zugeschaltete Maschine sofort nach dem 
Einschalten als Generator Last aufnimmt, muß man so parallelschalten, daß diese Maschine etwas übersynchron läuft, daß also ihre Frequenz etwas höher ist als die des Netzes (vgl. S. 5). Dies läßt sich allerdings nur bei verhältnismäßig ruhig laufenden Maschinen erreichen. Bei Generatoren, dic von Gasmaschinen angetrieben werden und die bei Leerlauf meistens sehr unruhig laufen, muß man zufrieden sein, wenn man die Maschinen überhaupt einigermaßen sicher parallelschalten kann. Die Zeitpunkte, die für das Parallelschalten geeignet sind, treten dann so kurz. zeitig auf, daß man schlechterdings keine weiteren Bedingungen an das Parallelschalten stellen kann, als daß die Maschinen ohne allzu derbe Stöße in Tritt kommen.

Bei der Schwierigkeit dieser Betriebsverhältnisse ist es wünschenswert, bei großen wertvollen Maschinen von der mehr oder minder großen Geschicklichkeit des Schalttafelwärters unabhängig zu werden. Man hat daher selbsttätige Apparate geschaffen, die unbeeinflußt von äußeren Nebenumständen fehlerlos den rich. tigen Zeitpunkt für das Parallelschalten wählen, ohne ihn jemals zu verpassen. Es würde zu weit führen, an dieser Stelle alle bekannten derartigen Einrichtungen zu besprechen, zumal diese als Spezialeinrichtungen nur für besondere Fälle in Frage kommen. Als besonders charakteristisches Beispiel ist im nachstehenden eine von Dr. Michalke angegebene selbsttätige Parallelschalteinrichtung der Siemens-Schuckert-Werke eingehend beschricben.

\section{b. Prinzip des Schaltmotors.}

Der Hauptteil der selbsttätigen Parallelschaltvorrichtung der S. S. W. ist ein kleiner asynchroner Drehstrommotor mit offener Wickelung im Ständer und kurzgeschlossener Einphasenwickelung im Läufer. Die Schaltung ergibt sich aus dem nachstehenden Schaltbild. Sie ist im Prinzip die gleiche wie die des auf S. 32 beschriebenen Lampenapparates, nur sind an die Stelle der Glühlampen die drei Wickelungsabteilungen des Ständers getreten. Die. Wirkungsweise der Schaltung folgt aus den Dingrammbildern auf S. 117. In diesen Bildern stellt der Stern $E_{1} E_{2} E_{3}$ die Sternspannungen des Netzes und $E_{1}^{\prime} E_{2}^{\prime} E_{3}^{\prime}$ die entsprechenden Sternspannungen der zuzuschaltenden Maschine dar. Dic Phasenverschiebung zwischen diesen beiden Spannungssystemen ist durch den Winkel $\varphi$ bezeichnet. Die in den einzelnen Wickelungen 
des Stënders auftretenden Spannungen sind durch die resultierenden Spannungen gegeben. Allerdings muß hierbei beachtet werden, daß die räumlich gleichgerichteten Spannungen elektrisch um $180^{\circ}$ verschoben sind, so daß tatsächlich die Differenz dieser Spannungen in den Wickelungen zur Wirkung kommt. Demgemäß würde an der einen Wickelung, die an gleichnamigen Polen angeschlossen ist, eine Spannung wirken, die durch $E_{2}-E_{2}^{\prime}$ dar-

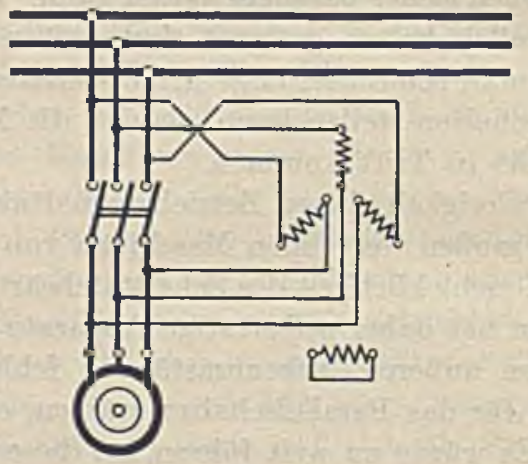

Bild 116.

gestellt wird. An den beiden anderen Wickelungen, die an vertauschten Polen liegen, würden die beiden Spannungen $E_{1}-E_{3}^{\prime}$ und $E_{3}-E_{1}^{\prime}$ wirken. Diese drei resultierenden Spannungen sind, wie aus den Diagrammbildern ersichtlich, in jedem Falle parallel zueinander. Die in den Wickelungen fließenden Ströme und demgemäß auch das erzeugte magnetische Feld stehen senkrecht auf diesen Spannungen. Das Feld ist im Diagramm durch den Pfeil $F$ angedeutet. Dieses im Ständer des Schaltmotors entstehende Feld ist also nicht, wie man erwarten könnte, ein Drchfeld, sondern cin einphasiges Wechselfeld. Ändert sich der Winkel $\varphi$, wie dies stets eintritt, wenn die beiden Drehstromsysteme nicht frequenzgleich sind, so ergeben sich für die verschiedenen ausgezeichneten Lagen die weiteren Diagrammbilder. Aus diesen folgt, daß die räumliche Lage des Wechselfeldes sich mit der Größe des Winkels $\varphi$ ändert. Bei einer vollen Verdrehung der beiden Drehstromsysteme gegeneinander, also bei $\phi=360^{\circ}$, dreht sich das Feld um $180^{\circ}$. Das Wechselfeld im Ständer dreht sich demgemäß mit der halben Geschwindigkeit der Phasen- 

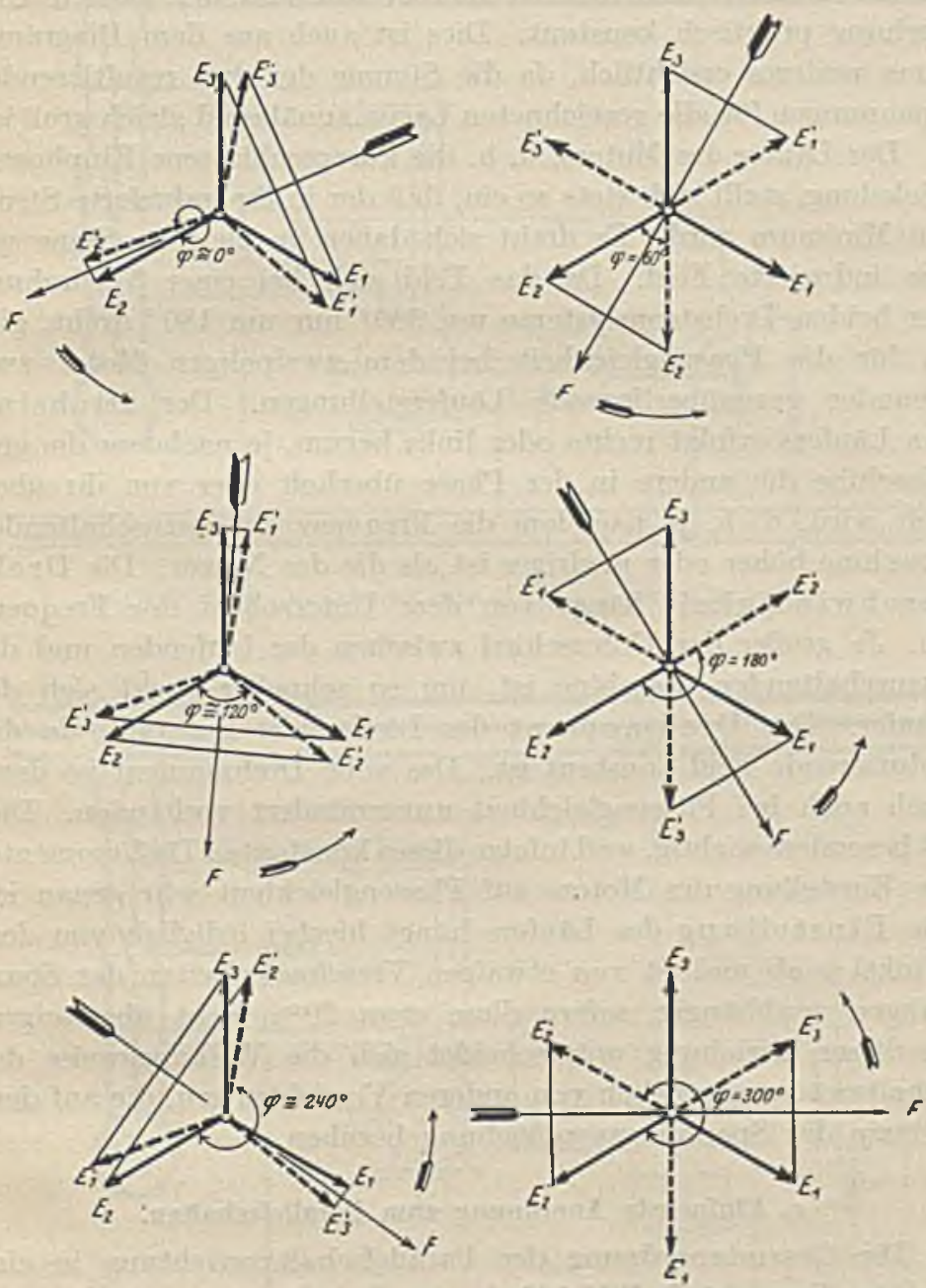

Bild 117 bis 122.

Tafel 25. Darstellung der elektrischen und magnetischen Vorhaltnisse im Schaltmotor. 
änderungen zwischen Netz und zuzuschaltender Maschine. Die Stärke des Wechselfeldes ist hierbei während der ganzen Umdrehung praktisch konstant. Dies ist auch aus dem Diagramm ohne weiteres ersichtlich, da die Summe der drei resultierenden Spannungen für alle gezeichneten Lagen annähernd gleich groß ist.

Der Läufer des Motors, d. h. die kurzgeschlossene Einphasenwickelung, stellt sich stets so ein, daß der in ibr induzierte Strom ein Minimum wird. Er dreht sich daher im gleichen Sinne wie das induzierte Feld. Da das Feld sich bei einer Verdrehung der beiden Drehstromsysteme um $360^{\circ}$ nur um $180^{\circ}$ dreht, gibt es für die Phasengleichheit bei dem zwcipoligen Motor zwei einander gegenüberliegende Läuferstellungen. Der Drehsinn des Läufers erfolgt rechts oder links herum, je nachdem die eine Naschine die andere in der Phase überholt oder von ibr überholt wird, d. h. je nachdem die Frequenz der zuzuschaltenden Maschine höher oder niedriger ist als die des Netzes. Die Dreh geschwindigkeit hängt von dem Unterschicd der Frequenz ab. Je größer der Unterschied zwischen der laufenden und der zuzuschaltenden Naschine ist, um so schneller dreht sich der Läufer. Das Drehmoment des Läufers ist konstant, da das induzierende Feld konstant ist. Das volle Drehmoment ist demnach auch bei Phasengleichheit unvermindert vorhanden. Dies ist besonders wichtig, weil infolge dieses konstanten Drehmomentes die Einstellung des Motors auf Phasengleichheit sehr genau ist. Die Einstellung des Läufers hängt hierbei lediglich von dem Winkel $p$ ab und ist von etwaigen Verschiedenheiten der Spannungen unabhängig, sofern diese etwa $20 \%$ nicht übersteigen. In dieser Beziehung unterscheidet sich die Wirkungsweise des Schaltmotors wesentlich von anderen Vorrichtungen, die auf dem Prinzip der Spannungsvergleichung beruhen.

\section{c. Elnfachste Anordnung zum Parallelschalten:}

Dic Gesamtanordnung der Parallelschaltvorrichtung in einfachster Form ist in Bild 123 dargestellt. Die Achse des Schaltmotors $M$ trägt eine Nockenscheibe $S$ mit zwei um $180^{\circ}$ gegen einander versetzten Einschnitten. Der gegen die Nockenscheibe mit Federkraft anliegende Kontakthebel $F$ wird daher bei jeder Umdrehung des Schaltmotors zweimal den Kontakt $K_{1}$ schließen. Der Kontakt $K_{1}$ liegt im Stromkreis einer Hilfsstromquelle $P N$ 




Bild 123. Schaltung mit einfachem Schaltmotor.

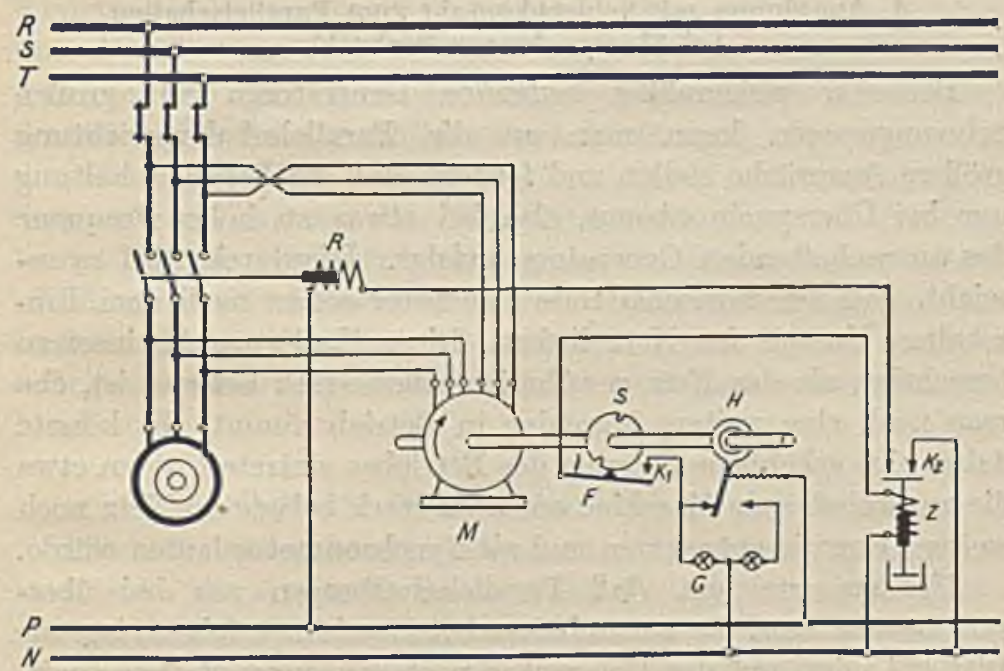

Bild 124. Schaltung mit Schaltmotor und Schleppkontakt zum Parallelsohalten bei übersynchroner Drebzahl.

Tafel 26. Finrichtung zum selbsttätigen Parallelschalten nach Dr. Michalke. 
und eines Zeitrelais $Z$ mit Uhrwerksverzögerung. Sobald durch den Kontakt $K_{1}$ der Stromkreis geschlossen wird, tritt das auf eine bestimmte Ablaufszeit cingestellte Uhrwerk des Zeitrelais in Tätigkeit. Ist der Stromkreis genügend lange geschlossen, d. h. erfolgt dio Umdrehung der Nockenscheibe des Schaltmotors genügend langsam, so erreicht das Zeitrelais seine Endstellung und schließt den Kontakt $K_{2}$. Hierdurch wird das Schaltrelais $R$ erregt, das den Hauptschalter zwischen Netz und zuzuschaltender Maschine einschaltet.

Diese einfache Anordnung wird vorzugsweise bei unruhig laufenden Maschinen benutzt. Sie kommt demnach in erster Linic bei Gasgeneratoren mit nicht zu großen Schwungmassen in Frage, die bei Leerlauf unruhig laufen und sich daher sehr schwer parallelschalten lassen. Man wird sich daher bei diesen Maschinen damit begnügen, lediglich bei Phasengleichheit parallel zu schalten, ganz gleichgültig, ob diese Phasengleichheit bei Überoder Untersynchronismus erreicht worden ist.

\section{d. Anordnung mit Schleppkontakt zum Parallelschalten bel übersynchroner Drehzahl.}

Bei sehr gleichmäßig laufenden Generatoren mit großen Schwungmassen kann man an die Parallelschaltvorrichtung größere Ansprüche stellen und fordern, daß die Parallelschaltung nur bei Übersynchronismus, also bei etwas zu hoher Frequenz des zuzuschaltenden Generators, erfolgt. Hierdurch wird es erreicht, daß der zuzuschaltende Generator sofort nach dem Einschalten Energie ins Netz liefert. Diese Forderung ist insofern berechtigt, als das Netz gewöhnlich schon stark belastet ist, ehe man noch eine weitere Maschine in Betrieb nimmt. Es könnte daher eine erhebliche Störung des Betriebes eintreten, wenn etwa die zuzuschaltende Maschine aus dem stark belasteten Netz noch weitere Energie entnehmen und als Synchronmotor laufen würde.

Um zu erreichen, daß Parallelschaltungen nur bei übersynchronem Lauf der zuzuschaltenden Maschine erfolgen, ist, wie Bild 124 zeigt, auf der Motorachse noch ein durch Reibung mitgenommener Schlepphebel $H$ angebracht, der zwischen zwei Kontakten spielt. Dieser Hebel legt sich je nach der Drehrichtung des Motors am rechten oder linken Kontakt an. Der Stromkreis des Zeitrelais $Z$ wird nun außer über den Kontakt $K_{1}$ noch über 
den linken Schlepphebelkontakt geführt. Bei der dem Übersynchronismus entsprechenden Rechtsdrehung des Schaltmotors wird dann der linke Kontakt des Schlepphebels dauernd geschlossen, so daß die Einschaltung des Zeitrelais lediglich von dem Kontakt $K_{1}$ abhängt. Bei Linksdrehung des Motors wird dagegen der Stromkreis des Zeitrelais dauernd unterbrochen und kann daher durch den Kontakt $K_{1}$ überhaupt nicht eingeschaltet werden. Die beiden Kontakte am Schlepphebel werden gleichzeitig noch dazu benutzt, um zwei verschiedenfarbige Signallampen $G$ einzuschalten. Läuft der zugeschaltete Generator zu schnell, also übersynchron, so leuchtet dic linke Lampe auf. Läuft er dagegen zu langsam, so brennt die rechte Lampe. Diese Signallampen geben daher ohne weiteres dem Maschinenwärter ein Zeichen, in welchem Simne die zuzuschaltende Maschine zu regeln ist.

Un die Verzögerung auszugleichen, die durch die Schaltvorrichtung und etwa noch zwischengeschaltete Schütze entsteht, muß das Kommando zum Parallelschalten stets etwas vor dem Eintritt der Phasengleichheit erfolgen. Dies ist dadurch erreicht, da $B$ die Nockenscheibe auf der Achse des Schaltmotors etwas gegen die der Phasengleichheit entsprechende Normalstellung verdreht ist. Die Scheibe schließt dann den Kontakt $K_{1}$ schon bevor die Phasengleichheit erreicht ist und unterbricht ihn etwa in dem Augenblick, wo die Phasengleichheit eben überschritten wird. Für die Rückwärtsdrehung des Schaltmotors steht dann allerdings die Nockenscheibe in einer falschen Stellung, aber dies ist belanglos, da dann der Schlepphebel an dem rechten Kontakt anliegt, so daß der Stromkreis des Zeitrelais dauernd unterbrochen ist.

\section{c. Selbsttuitige Regelung der Antriebsmaschine.}

Die in vorhergehenden Abschnitt beschriebene Einrichtung kann auch dazu benutzt werden, um die Antriebsmaschine der an das Netz anzuschließenden Maschine selbsttätig im richtigen Sinne zu regeln und auf die synchrone Drehzahl zu bringen. Hierbei ist es an sich gleichgültig, ob die Antriebsmaschine eine Kolbendampfmaschine, eine Dampfturbine, ein Gasmotor, ein Ólmotor oder endlich der Elektromotor eines Umformers ist. In Bild 125 auf S. 123 ist die Gesamtschaltung der Einfachheit halber für einen 
Gleichstrom-Drehstromumformer angegeben. Parallel zu den beiden Signallampen $G$ sind die beiden Steuerrelais $R_{1}$ und $R_{2}$ angeschlossen. Das Relais $R_{1}$ wird dann eingeschaltet, wenn Ubersynchronismus vorhanden ist, während das Relais $R_{2}$ bei Untersynchronismus eingeschaltet wird. Durch diese Steucrrelais werden die Relaiskontakte $U_{1}$ und $U_{2}$ betätigt. Bei der eingezcichneten Drehrichtung des Schaltmotors $M$ legt sich der Schlepphebel $H$ links an. Beim Schließen des Kontaktes $K_{1}$ wird dann das Steuerrelais $R_{1}$ erregt und zieht seinen Anker an, so da $\boldsymbol{B}$ der obele Kontakt von $U_{1}$ geschlossen wird. Das Relais $R_{2}$ ist dagegen stromlos, so daß der untere Kontakt von $U_{2}$ geschlossen bleibt. Der Anker des als Hilfsmotor dienenden kleinen Hauptstrommotors wird daher von unten nach oben vom Strom durchflossen. Der Hilfsmotor dreht sich daher in dem entsprechenden Drehsinne und beeinflußt durch Regelung des Feldwiderstandes den Antriebsmotor der zuzuschaltenden Maschine etwa in verlangsamendem Sinne. Die zuzuschaltende Drehstrommaschine läuft infolgedessen jetzt etwas zu langsam. Der Schaltmotor kehrt scine Richtung um und der Schlepphebel $H$ legt sich an den rechten Kontakt an, so daß das Relais $R_{\mathbf{2}}$ nunmehr eingeschaltet ist, während $R_{1}$ stromlos wird. Infolgedessen wird der obere Kontakt von $U_{2}$ und der untere Kontakt von $U_{1}$ geschlossen. Hierdurch wird der Strom im Anker des Hilfsmotors umgekehrt; der Hilfsmotor ändert daher seine Drehrichtung und beeinflußt den Antriebsmotor der zuzuschaltenden Maschine in beschleunigendem Sinne. Wie aus dem nebenstehenden Schaltbild hervorgeht, ist in den Stromkreis des Hilfsmotors ein Widerstand $W$ eingeschaltet, wenn die oberen Kontakte des Umschalters $U_{1}$ geschlossen sind. Der Hilfsmotor dreht sich daher in der einen Richtung langsamer als in der anderen Richtung. Würde der Motor nach beiden Richtungen gleich schnell laufen, so würde bei völligem Synchronismus ein daucrndes Pendeln des Schaltmotors $M$ um eine Stellung eintreten können, bei der voraussichtlich keine Phasengleichheit stattfindet. Durch die absichtlich eingeführte Ungleichheit in der Reguliergeschwindigkeit tritt das Pendeln um eine stetig fortschreitende Phasenstellung ein, so daß nach kurzer Zeit eine Phasenstellung erreicht wird, bei der das Parallelschalten ausgeführt werden kann. Diese Einrichtung bewirkt also durch die Pendelerscheinungen das, was 


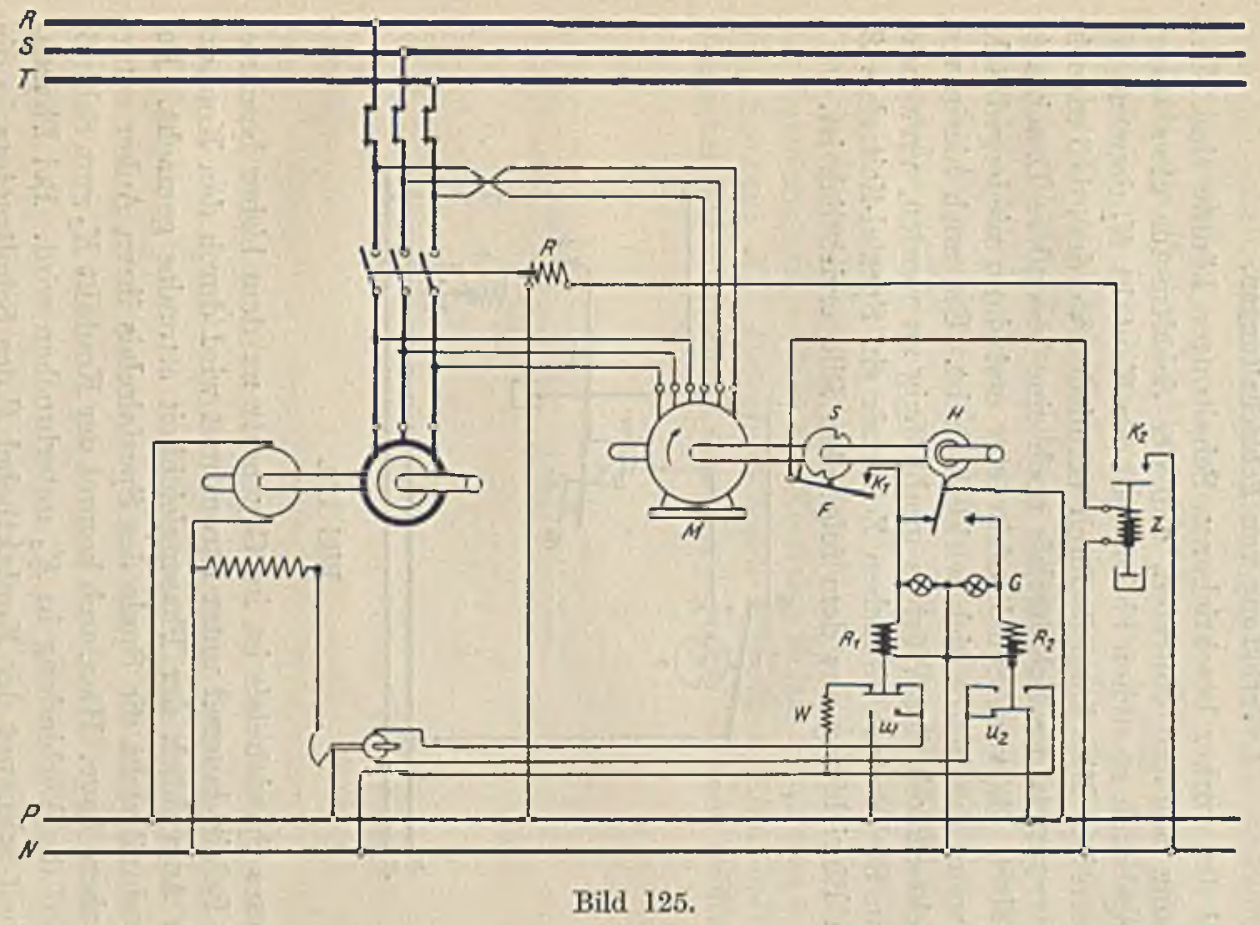

Tafel 27. Einrichtung zum selbsttütigen Parallelschalten mit selbsttiitiger Regelung der Antriebsmaschine. 
sonst der Wärter von Hand machen würde. Sie gibt immer nur Regelungsstöße im richtigen Sinne und macht fortgesetzt den Versuch, den Generator auf Synchronismus zu bringen.

\section{f. Verhütung ron Fohlschaltungen.}

Bei den vorher beschriebenen Schaltungen könnte eine Fehlschaltung dadurch eintreten, daß der Schaltmotor etwa durch Leitungsbruch so stehen bleibt, da.B der Kontakt $K_{1}$ dauernd geschlossen ist. Dies kann dadurch vermieden werden, daß man in den Stromkreis des Schaltrelais noch eino besondere Drucktaste einschaltet, die vom Schalttafelwärter erst dann niedergedrückt wird, wenn der Motor sich wirklich dreht. Um auch hierin von der Achtsamkeit des Wärters unabhängig zu werden, verwendet man an Stelle eines einfachen Zeitrelais ein Spezial-Zeitrelais $Z$, dessen Einrichtung aus dem folgenden Bild ersichtlich ist.

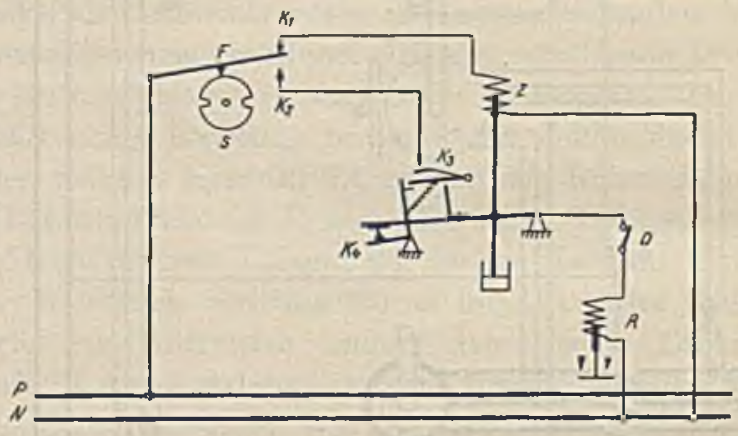

Bild 126.

Dieses Spezialrelais ist im Gegensatz zu dem bisher beschriebenen Relais dauernd unter Strom und wird durch den Kontakt $K_{1}$ im Augenblick der Phasengleichheit stromlos gemacht. Bei Stromschluß zieht die Spule des Spezialrelais ihren Anker sofort in die obere Lage. Hierdurch kommt der Kontakt $K_{3}$ zum Schluß, während die Verbindung in $K_{4}$ unterbrochen wird. Bei Phasengleichheit schnappt der Kontakthebel $F$ des Schaltmotors in die Einschnitte der Scheibe, so daß der Kontakt $K_{1}$ unterbrochen und der Kontakt $K_{2}$ geschlossen wird. Durch das Unterbrechen des Kontaktes $K_{1}$ wird das Spezialzeitrelais stromlos, sein Eisenkern sinkt langsam entsprechend der Zeiteinstellung des Relais 
herab. Hierdurch wird, falls die Stromunterbrechung, also die Phasengleichhoit lange genug dauert, der Kontakt $K_{4}$ geschlossen, während gleichzeitig der federnde Kontakt $K_{3}$ noch bestehen bleibt. Dann ist über die Kontakte $K_{2}, K_{3}, K_{4}$ und $D$ der Strom. kreis des Schaltrelais $R$ eingeschaltet, so daß die Parallelschaltung vollzogen wird.

Hält die Phasengleichheit nur kurze Zeit an, so wird der Stromkreis des Schaltrelais schon wieder bei $K_{2}$ unterbrochen, ehe der Kontakt bei $K_{4}$ hergestellt ist. Das Schaltrelais kann daher in diesem Falle nicht in Tätigkeit treten. Unmittelbar nach der Unterbrechung von $K_{2}$ wird aber schon wieder der Kontakt $K_{1}$ geschlossen, das Zeitrelais zieht seinen Anker wicder an, und das Spiel beginnt von neuem. Solange keine Phasengleichheit besteht, ist der Kern des Spezialrelais $Z$ stets angezogen. Tritt in dieser Lage ein Drahtbruch oder unsicherer Kontakt in der Zuleitung auf, so fällt der Kern des Relais herab und schließt den Kontakt $K_{4}$. Hierdurch kann aber in keinem Falle eine Fehlschaltung erfolgen, da daun der Kontakt $K_{2}$ noch geöffnet ist. 


\section{Schaltungskontrolle.}

a. Kontrolle auf richtiges Drehfeld.

Vor dem endgültigen Anschließen einer Drehstrom-Maschine ermittelt man zunächst den Richtungssinn des von ihr erzeugten Drehfeldes. Dieses muß den gleichen Richtungssinn wie das vom Netz bzw. von den anderen laufenden Maschinen erzeugte Drehfeld haben. Un dies festzustellen, öffnet man den Maschinenschalter und schließt $\mathrm{z}$ wischen die drei Klemmenpare des Schalters eine Anzahl Glühlampen an, wie Bild 127 zeigt. Die Lampenzah!

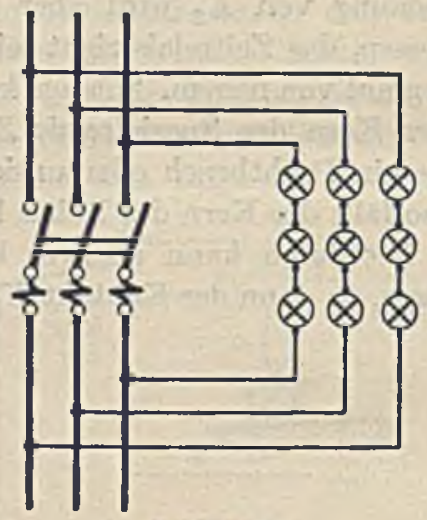

Bild 127.

muß so groß sein, daß jede in Reihe geschaltete Gruppe eine Spannung aushalten kann, die um $15 \%$ höher als die Netzspannung liegt. Dann schließt man die Maschine an die Naschinenkabel an und setzt sie in Betrieb. Bei richtigem Drehfeld und annähernd synchroner Drehzahl der Maschinen müssen die drei Lampengruppen gleichzeitig aufleuchten und verlöschen. Leuchten die Lampen nicht gleichzeitig, sondern der Reihe nach auf, wie es auf S. 16 beschrieben ist, so folgt daraus, da $B$ das Drehfeld der zuzuschaltenden Maschine in anderem Richtungssinn umläuft wie das des Netzes. Um dies richtig zu stellen, müssen zwei Anschlußkabel an der Maschine vertauscht werden. Bei Hochspannung kann man die Anzahl der für diese Kontrollschaltung erforderlichen Lampen dadurch beschränken, daB man die Versuche bei unerregten Maschinen vornimmt. Man hat in diesem 
Falle nur mit einer geringen Remanenzspannung von etwa 5 bis $10 \%$ der Betriebsspannung zu rechnen. Ist es nicht möglich, die Anlage mit unerregten Maschinen laufen zu lassen, so benutzt man drei für die Netzspamnung bemessene Spannungswandler, die allerdings bei dieser Schaltung um $15 \%$ überlastet werden, und schaltet nach dem folgenden Schaltbild. Auch die Herstellung

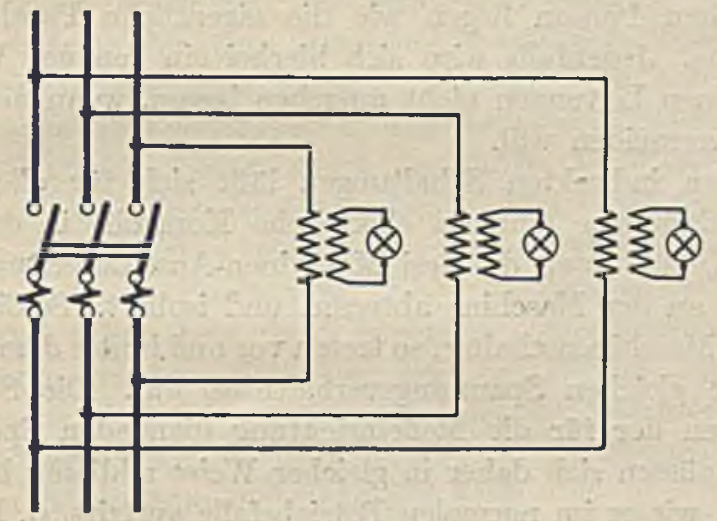

Bild 128.

dieser Schaltung wird kaum Schwierigkeiten machen, da Spannungswandler für die Netzspannung wohl stets in genügender Zahl vorhanden sein werden. An Stelle der Glühlampen kann man zur Kontrolle des Drchfeldes auch einen Drehfeldrichtungsanzeiger oder schließlich auch einen beliebigen Asynchronmotor benutzen. Der Drehfeldrichtungsanzeiger muß ebenso wie der Asynchronmotor im gleichen Drehsinn umlaufen, wenn cr bei gleichsinvigem Anschluß vom Netz oder von der zuzuschaltenden Maschine gespeist wird.

\section{b. Kontrolle auf richtige Schaltung.}

Bei den dirckten Schaltungen läßt sich eine für alle Fälle geltende elektrische Kontrolle nicht ohne weiteres anstellen. Bei Dunkelschaltung kamn man zwar die im vorhergehenden Abschnitt angegebene Schaltung benutzen, jedoch treten hierbei an den drei Lampengruppen verschiedene Spannungen auf, so daß die eine Lampenreihe unter Umständen etwas überlastet wird. Aber immerhin müssen auch dann die drei Iampengruppen gleichzeitig und zugleich mit der Phasenlampe verlöschen, und das 
verwendete Anzeigeinstrument mu $B$ auf Synchronismus zeigen. Bei Hellschaltung kamn jedoch diese einfache Schaltung nicht verwendet werden, da die eigentliche Parallelschalteinrichtung durch diese Kontrollschaltung gestört würde. Man könnte höchstens auch an den Schalterkontakten eine ein phasige Hellschalt ung nach dem Bild 6 auf S. 10 ausführen, jedoch müßte diese an den gleichen Phasen liegen wie dic eigentliche Parallelschalteinrichtung. Jedenfalls wird sich hierbei ein genques Verfolgen der einzelnen Leitungen nicht umgehen lassen, wenn man Trugschlüsse vermeiden will.

Bei den indirekten Schaltungen läßt sich für alle Schaltmöglichkeiten eine einfache elektrische Kontrolle in der Weise ausführen, daß man dio drei Maschinen-Anschlußleitungen unmittelbar an der Maschine abtrennt und isoliert. Schließt man dann den Maschinenschalter, so treten vor und hinter dem Schalter genau die gleichen Spannungsverhältnisse auf. Die Sekundärspannungen der für die Meßeinrichtung dienenden Spannungswandler müssen sich daher in gleicher Weise addieren bzw. subtrahieren, wie es im normalen Betriebsfalle auftritt, $d$. h. die an dio Sekundärseite der Meßwandler angeschlossenen Meßinstrumente müssen den Augenblick des Parallelschaltens anzeigen. Bei Dunkelschaltung müssen daher die Phasenlampen verlöschen und der Nullspannungsmesser auf Null zeigen, während bei Hellschaltung dic Phasenlampen hell brennen müssen und der Summenspannungsmesser über der roten Kennmarke einspielen muß. Wird an Stelle eines Null- oder Summenspannungsmessers ein Synchronoskop benutzt, so muß dieses sich auf die dem Synchronismus entsprechende rote Marke fest einstellen. Etwaige Schaltfehler ergeben sich bei dieser Kontrolle ohne weiteres. Zeigt sich z. B., daß bei Dunkelschaltung die Phasenlampen mit geringer Lichtstärke leuchten und der Nullspannungsmesser etwa dic halbe Spannung anzeigt, so läßt dies darauf schließen, daß der Maschinenspannungswandler primär an eine falsche Phase angeschlossen ist. Leuchten andererseits die Phasenlampen mit voller Spannung und zeigt der Nullspannungsmesser die volle Spannung an, so müssen die Anschlüsse auf der Sekundärseite des Maschinenspannungswandlers vertauscht werden. 


\section{Elektrische Befehlsübertragung zwischen Schaltbühne und Maschinenraum.}

\section{n. Allgemeines.}

Die für das Parallelschalten erforderliche genaue Einregelung der Maschinen setzt voraus, daß zwischen der Schaltbühne und den einzelnen Maschinen eine gute Verständigung möglich ist. Bei den früheren kleineren Kraftwerken war es vielleicht möglich, sich durch Zurufe oder Winke, vielleicht auch durch einfache Glockensignale zu verständigen. Bei den modernen großen Kraftwerken wird dies jedoch durch die Größe der Maschinenräume und durch das Geräusch der laufenden Maschinen praktisch unmöglich. Die Zcichen würden in vielen Fällen ganz überhört oder, was ebenso schlimm ist, falsch verstanden werden. Man kam daher schon bald dazu, besondere Signalanlagen zur Verständigung zwischen Maschinen und Schaltbühne zu benutzen, um so mehr, als bei den neueren Anlagen die Schalteinrichtungen zumeist nicht mehr im Maschincnraum, sondern in einem besonderen Schaltraum untergebracht sind.

Die Signaleinrichtungen werden entweder als Glühlampentafeln oder als Zeigerapparate ausgeführt. Die Zeigerapparate lehnen sich in ihrer Bauform eng an die Kommandoapparate an, die auf Schiffen in größtem Umfange benutzt werden und sich durch ihre große Betriebssicherheit auszeichnen. Da die Ausführungsformen dieser Signaleinrichtungen wenig beliannt sind, sollen sie im nachstehenden etwas eingehender besprochen werden.

\section{b. Glühlampentafeln.}

Die Glühlampentafeln bestehen aus einer Reihe von Transparentscheiben, die durch einzelne Glühlampen wahlweise beleuchtet werden können. Die Transparentscheiben tragen die Kommandoaufschriften "Anlassen", "Schneller", „Langsamer.", "Gut", „Abstellen" und die Bestätigung des ausgeführten Befehls, „Fertig“. Bei Anlagen mit Wasserturbinen kommt noch ein Notsignal „Maschine in Gefahr" hinzu. Sämtliche

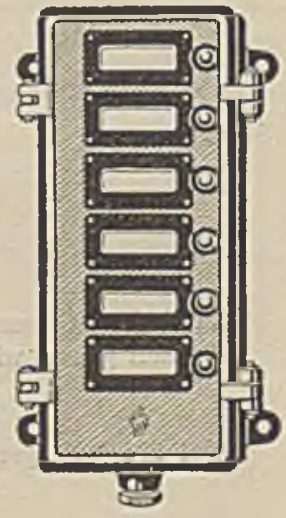

Bild 129.

Skirl, Parallelschalten. 2, Aufl. 
Gebor

Empfänger

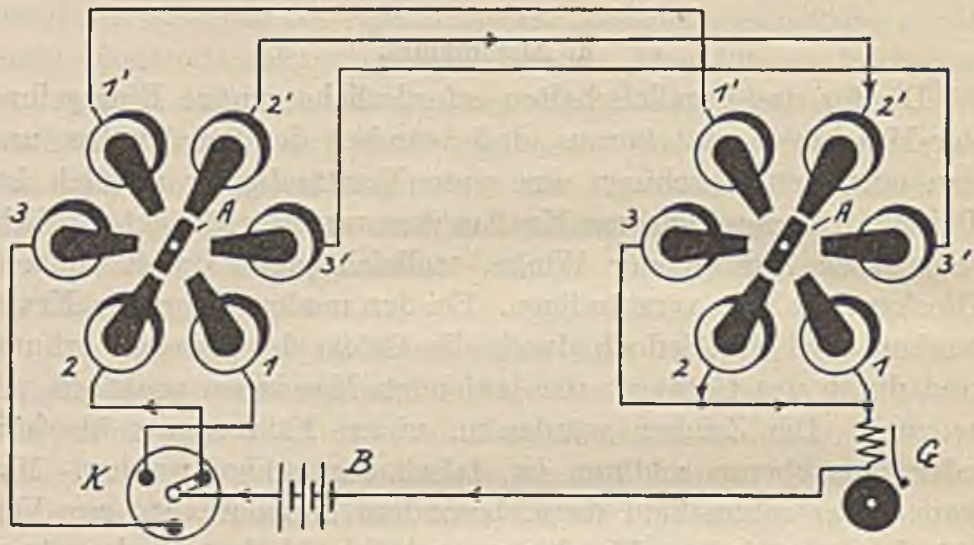

Bild 130. Innero Schaltung. Die gegenüberliogenden Spulen sind paarweise derart in Roiho geschaltet, da $B$ sio einander ungloichnamige Polo zuwenden.

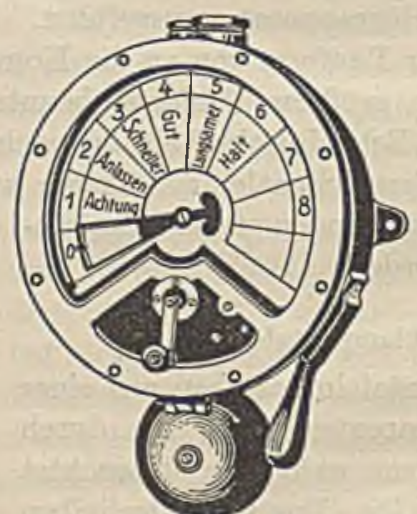

Bild 131. Gebeapparat mit Kurbelantricb. Der Empfänger iat äußerlich genau wio der Geber aufgebaut, joloch fällt boi diesem dio Kurbel weg.

Tafel 28. Zeiger-Belehlsapparnt mit Sechsspulen-Motor, für Gleichstrom. 
Aufschriften sind nur sichtbar, wenn die dahinter befindlichen Glühlampen eingeschaltet sind.

Die Arbeitsweise einer derartigen Signaleinrichtung ist außerordentlich einfach. Soll ein Befehl gegeben werden, so gibt der Schalttafelwärter zunächst mit der Ruftaste ein Glockenzeichen als Achtungssignal. Darauf schaltet er das mit dem entsprechenden Befehl bezeichnete Signal ein. An der Glühlampentafel im Maschinenraum leuchtet dann das zugehörige Befehlsfeld gleichzeitig mit einem entsprechenden Kontrollfeld im Schaltraum auf. Die Lampen brennen an beiden Stellen so lange, bis der Maschinist mit der Quittungstaste den Befehl bestätigt. Hat der Maschinist den erhaltenen Befehl ausgeführt, so drückt er die Fertigtaste. Es leuchten dann die Lampen des Fertigsignals sowohI im Schaltraum als auch zur Kontrolle an der Lampentafel auf. Außerdem ertönt im Schaltraum eine Rasselglocke so lange, bis dort die Quittungstaste niedergedrückt wird. Besteht Gefahr für die Maschine, so gibt der Maschinist das Notsignal ,Maschine in Gefahr". Auch hierbei leuchten die zugehörigen Lampen sowohl an der gebenden wie an der Empfangsstelle auf, bis das Signal mit der Quittungstaste abgestellt und dadurch bestätigt wird.

\section{c. Zeiger-Befehlsapparat mit Sechsspulen-Motor, für Gleichstrom.}

Das Triebwerk dieses von $\mathrm{S}$. \& $\mathrm{H}$. gebauten Apparates besteht aus sechs im Kreise angeordneten Elektromagneten, deren Kerne radiale Polschuhe besitzen ( $v$ gl. Bild 130). Zwischen diesen Polschuhen ist ein kleiner Anker $A$ aus weichem Eisen drehbar gelagert, der unter Zwischenschaltung einer Schnecke den Zeiger antreibt. Die gegenüberliegenden Spulen sind paarweise derart in Reihe geschaltet. da $B$ sie einander ungleichnamige Pole zuwenden. Die drei Spulenpaare können durch einen kleinen Kurbelschalter $K$ wahlweise in den Stromkreis der Batterie $B$ eingeschaltet werden. Die Wirkungsweise der Einrichtung ist dann folgende: Steht die Kurbel in der eingezeichmeten Stellung, so werden in Geber und in Empfänger die Spulenpaare 2 und 2 rom Strome durchflossen. Die Anker stellen sich dann in dic Richtung der Polverbindungslinie ein. Dreht man dic Schalterkurbel auf den nächsten Kontakt, so werden die Spulenpaare 3 und 3 ' eingeschaltet und der Anker stellt sich wieder in die entsprechende Pollinie ein. Bei einer vollen Kurbel- 
umdrehung dreht sich der Anker ruckweise um $180^{\circ}$. Es sind also zwei Kurbelumdrehungen für eine volle Umdrehung der Anker erforderlich. Um eine möglichst große Anzahl von Befehlen auf einer Skala unterzubringen, ist die Utbertragung zwischen Anker und Zeiger so gewählt, daß der Zeiger bei jeder vollen Kurbelumdrehung $u m$ ein Skalenfeld vorrückt. Hierbei kann man an der oberhalb der Kurbel des Geberapparates angeordneten Skala stets unmittelbar ablesen, welcher Befehl gegeben worden ist (vgl. Bild 131). Gleichzeitig mit dem optischen Sigual liann auch ein akustisches Signal, z. B. durch einen Einschlagwecker, gegeben werden, so da $\beta$ bei jedem Zeigersprung von einem Befehlsfeld zum anderen ein Glockenschlag ertönt. Um es zu errcichen, daß die Anzahl der Glockenschläge der Nummer des Befehlsfeldes entsprechen, muß der Zeiger des Geberapparates vor Beginn jeder Befehlsabgabe stets auf das Nullfeld zurückgekurbelt werden. Durch eine besondere Vorrichtung wird es hierbei erreicht, daß der Wecker beim Zurückkurbeln nicht ertönt. Die Speisung der Einrichtung erfolgt durch Gleichstrom bis 220 Volt.

Diese einfache und älteste Vorrichtung ist insofern noch nicht vollkommen, als die gegenseitige Lage der Zeiger des Geber- und Empfangsapparates nicht eindeutig ist. Wenn z. B. durch äußeren Eingriff der Zeiger des Empfangsapparates gegenüber dem des Geberapparates rerstellt ist, wie es bei der Montage vorkommen kann, so wird er beim Einschalten des Stromes nicht ohne weiteres in die richtige vom Geberapparat vorgeschriebene Stellung gehen, da der unpolarisierte Anker des Sechsspulenmotors auch in die um $180^{\circ}$ verdrehte Lage einspringen kann. Man muß daher vor der Inbetriebnahme die Geber- und Empfangsapparate erst in Utbereinstimmung bringen. Dies geschieht in einfacher Weise dadurch, daß man den Geberapparat von der Nullage in die Endstellung und dann wieder zurück auf Null kurbelt. Diese Unbequemlichkeit wird bei den folgenden Einrichtungen vermieden.

\section{d. Zciger-Befehlsapparat mit Dreispulen-Anker, für Gleichstrom.}

Der Empfänger dieser ebenfalls von S. \& H. gebauten Einrichtung besteht aus einem Trommelanker mit drei in Dreieckschaltung verbundenen Spulen. Der Trommelanker ist in einem zweipoligen, durch eine Nebenschlußwickelung erzeugten Nagnet- 


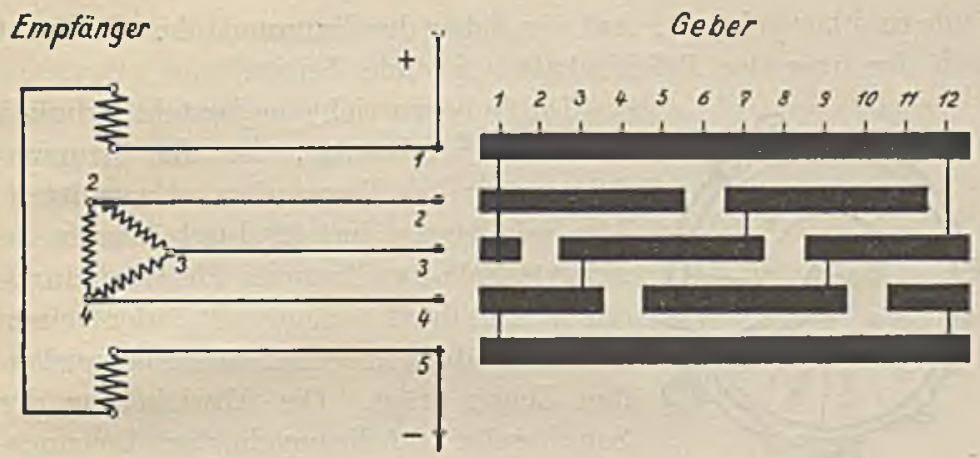

Bild 132. Innenschaltung mit abgowickelter Schaltwalzo.
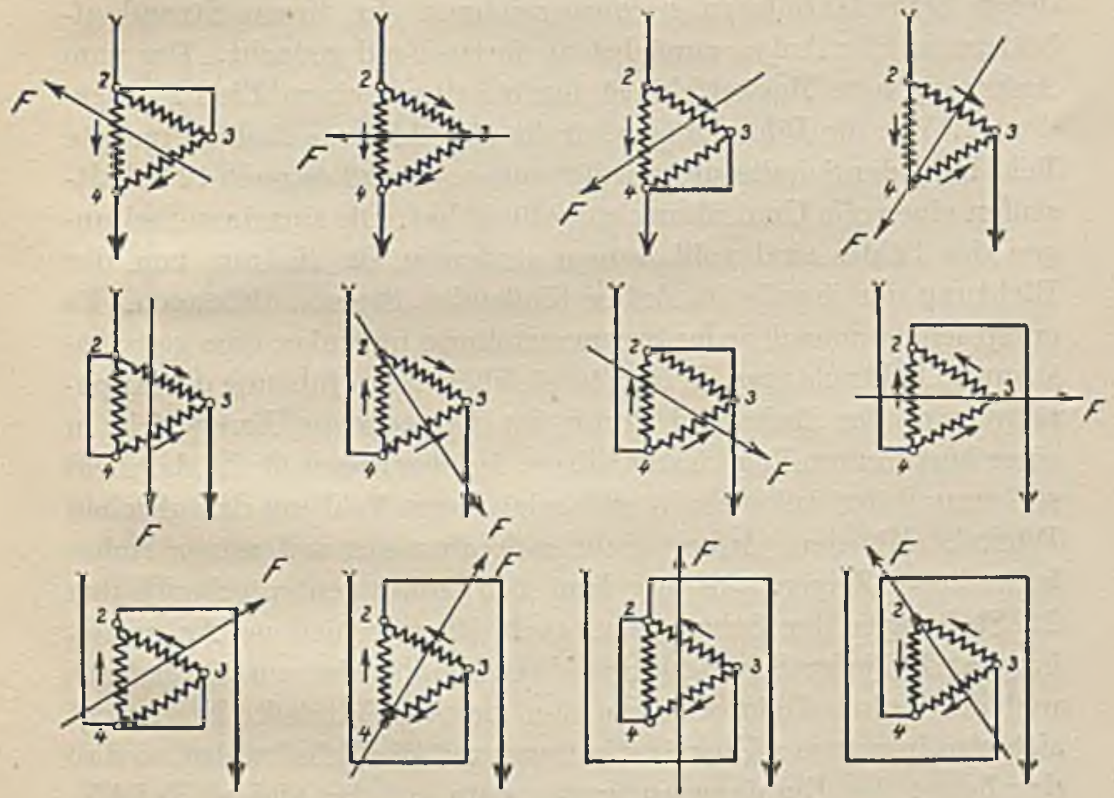

Bild 133. Schaltstufen für dio versehicdenen Zeigerstellungen des Gebers.

Tafel 29. Zeiger-Befehlsapparat mit Dreispulen-Anker, fïr Gleichstrom. 
felde drehbar gelagert. Auf der Achse des Trommelankers befindet sich der über der Befehlsskala spielende Zciger.

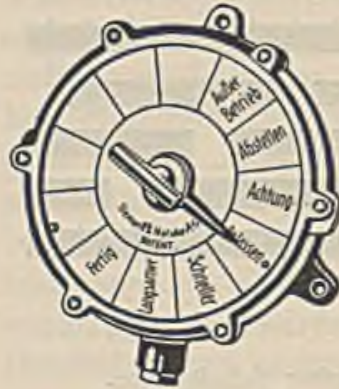

Bild 134.

Die Gebervorrichtung besteht lediglich in einer Schaltwalze, die die Stromzuführung zu dem 'Trommelanker vermittelt. Die Schaltwalze hat 12 durch Rasten gesicherte Schaltstellungen. Sie wird durch einen Handgriff eingestellt, der einen ebenfalls über einer Befehlsskala spielenden Zeiger trägt. Die Abwickelung der Schaltwalze und die zugehörigen Leitungsverbindungen sind auf Tafel 29 dargestellt. Zum leichteren Verständnis sind darunter noch die einzelnen Schaltstufen in besonderen Stromlaufbildern herausgezeichnet. In diesen Stromlaufbildern ist der Anker zunächst als feststehend gedacht. Das vom Anker crzeugte Magnetfeld ist hierbei durch einen Pfeil $F$ angedeutet. Wie die Bilder zeigen, dreht sich das vom Anker erzeugte Feld bei jeder Schaltstufe um $30^{\circ}$ weiter, so daß es nach 12 Schaltstufen eine volle Umdrehung ausgeführt hat. Die einzelnen Stellungen des Feldes sind vollkommen eindeutig, da sie nur von der Richtung der jeweils iun Anker fließenden Ströme abhängen. Es entspricht demnach jeder Stromverteilung im Anker eine ganz bestimmte Feldrichtung. Bei der tatsächlichen Ausführung des Apparates wird das Ankerfeld durch das feststehende Erregerfeld in einer bestimmten Lage festgehalten. Infolgedessen bleibt das Feld stehen und der Anker dreht sich relativ zum Feld um den gleichen Winkel. Mit dem Anker dreht sich aber der auf seiner Achse befindliche Zeiger. Es ergeben sich somit entsprechend den 12 Stellungen der Schaltwalze auch 12 verschiedene Ankerstellungen. Bei jeder Schaltstufe wird die Schaltwalze um $30^{\circ}$ gedreht und der Anker dreht sich um den gleichen Winkel. Er bewegt sich also in genauer U'bereinstimmung mit der Schaltwalze, so daß der Zciger des Empfangsapparates stets auf das gleiche Befchlsfeld zeigt wie der Zeiger des Geberapparates. Zum Betriebe der Einrichtung dient Gleichstrom von 30 Volt Spannung. Bei Verwendung eines entsprechenden Vorwiderstandes kann die Einrichtung jedoch auch an ein. Gleichstrom-Lichtnetz angeschlossen werden. 
Da bei diesem System dic Lage des Ankers des Empfangsapparates eindeutig durch die Richtung der in ihm fließenden Ströme bestimmt wird, ist bei diesem Apparat auch durch äußeren Eingriff eine Störung der UUbereinstimmung der beiden Zeiger nicht möglich. Die Zeiger werden sich vielmehr sofort nach Einschalten des Stromes selbsttätig auf das gleiche Befehlsfeld einstellen. Da jeder Schaltstufe ein Drehwinkel der Schaltwalze um $30^{\circ}$ entspricht, sind bei diesem Apparat nur 12 verschiedene Befehlsübertragungen möglich.

\section{e. Zeiger-Bofehlsapparat nach dem Wechselstromsystem.}

Geber

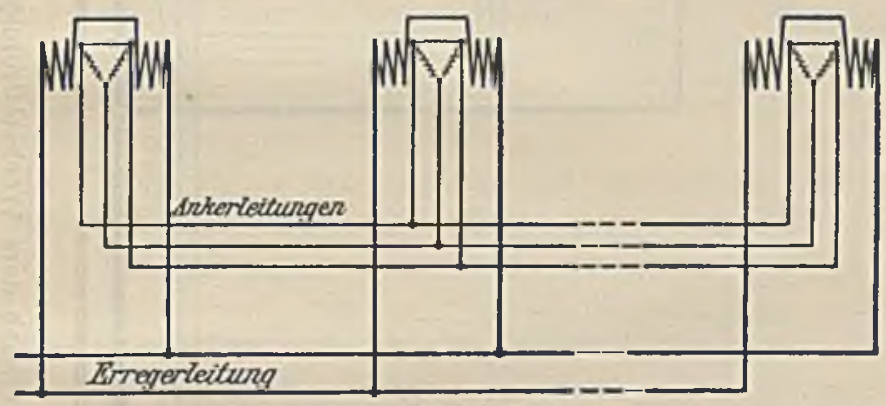

Bild 135.

Bei dem Wechselstromsystem von S. \& H. sind Geber und Empfänger vollkommen gleich aufgebaut. Die grundsätzliche Anordnung geht aus der nachstehenden Tafel 30 hervor. Jeder Apparat bestcht aus einem zweipoligen, aus geblättertem Eisen aufgebauten Polgestell $P$ und einem dreiphasig gewickelten Anker $A$. Die Erregerwickclungen $F$ der beiden Polgestelle werden in Nebeneinanderschaltung mit 50 Volt Einphasenstrom gespeist. Die in diesen Polgestellen erzeugten Wechselfelder sind demnach in Phase. Die beiden dreiphasigen Anker sind über je drei Schleifringe miteinander gleichpolig verbunden. Sind die beiden Anker in genau gleicher Lage, so werden in ihren Wickelungen genau die gleichen Elektromotorischen Kräfte induziert. Sic heben einander auf, da die Anker gegeneinander geschaltet sind. Wird der eine der beiden Anker, z. B. der Anker des Gebers, gedreht, so ändern sich die in seinen Spulen induzierten Elektro- 


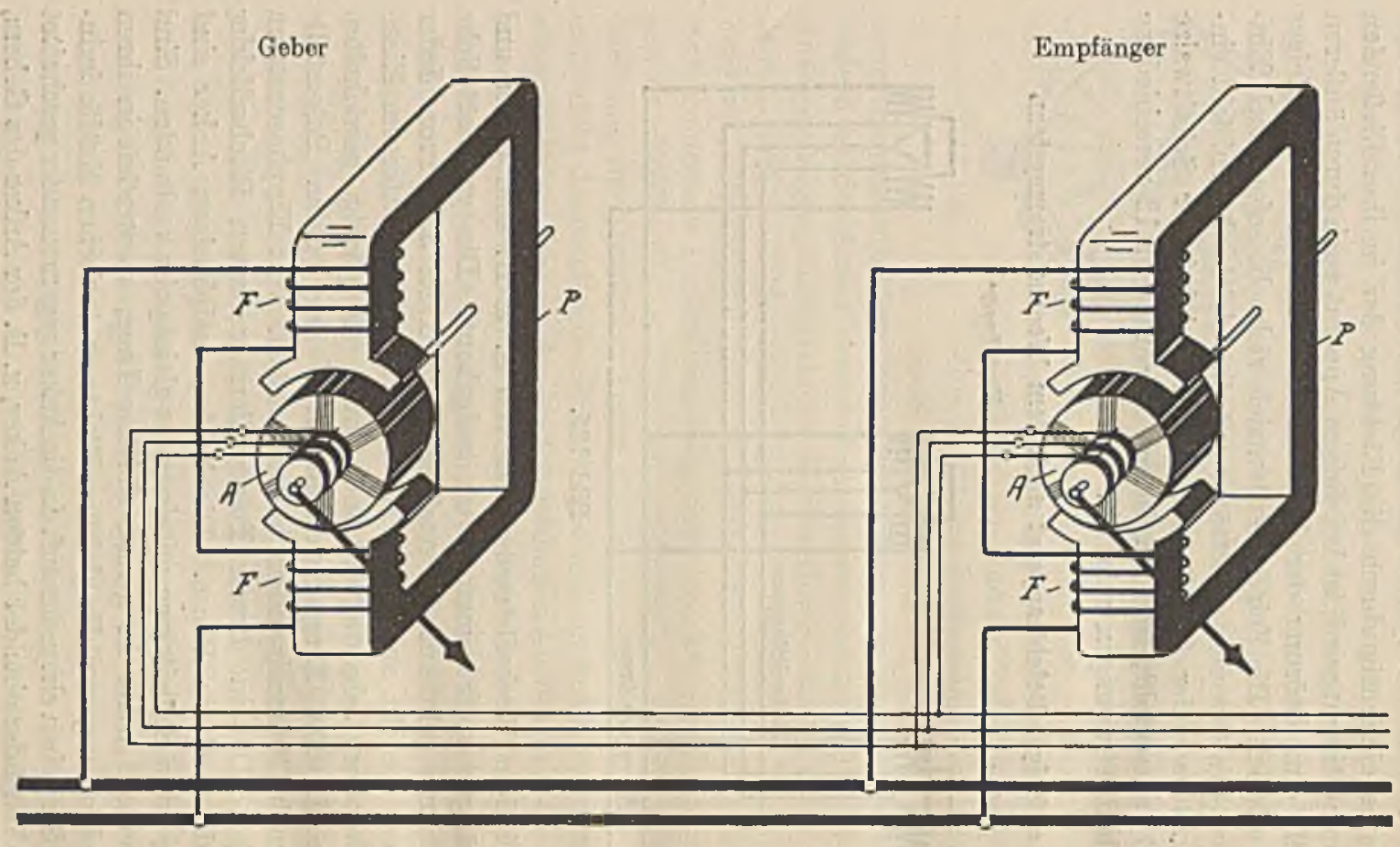

Tafel 30. Zeiger-Befohlsapparat nach dem Wechselstromsystem. (Bild 136.) 
motorischen Kräfte. In den Verbindungsleitungen der beiden Anker entstehen daher Ausgleichströme, die den Anker des Empfängers so lange drehen, bis dié dort induzierte Elektromotorische Kraft gleich der des Gebers geworden ist, also bis kein Ausglcichstrom mehr fließt. Dies wird aber erst dann erreicht, wemn der Anker des Empfängers genau die gleiche Stellung gegenüber den Polen einnimmt. Die beiden Anker werden demgemäß unter dieser Wechselwirkung stets die gleiche relative Lage gegenüber den Magnetpolen einnehmen, $d, h$. die Zeiger werden stets auf das gleiche Befehlsfeld zeigen.

In der Ausführung unterscheiclen sich Geber und Empfänger zunächst darin, daß die Ankerachse des Gebersystems von außen verstellt werden kann, während die Ankerachse des Empfängersystems frei beweglich ist. Um cin schwankungsfreies Einstellen des Zeigers zu exzielen, ist das Empfängersystem mit ciner clektromagnetischen Dämpfung versehen.

Mit einem Geber kömmen, wie das Bild auf S. 135 zeigt, bcliebig viele Empfänger verbunden werden, ohne daß dadurch die Zahl der Leitungen vcrmehrt würde. In jedem Falle sind fünf Leitungen, und zwar zwei für die Felderregung und drei für die Anker erforderlich. Die Empfängersysteme liaben hierbei stets die gleiche Größe, während das Gebersystem je nach der Anzahl der zu betreibenden Empfänger verschieden bemessen. scin muß.

Der Zeigerbefehlsapparat nach dem Wechselstromsystem ist die vollkommenste Art der elelitrischen Befehlsübertragungen. Dio Zeiger stellen sich sofort beim Einschalten des Stromes richtig ein, da jeder Lage des Geberankers nur eine Stellung des Empfängerankers entspricht. Die Einstellung der Zeiger erfolgt energisch und daher sicher. Die Anzahl der zu übertragenden Befehle kann beliebig groß sein, dabei ist die Schaltung äußerst einfach und übersichtlich und durch Fortfall jeder Stromunterbrechungsvorrichtung sehr betriebssicher. Wit dem Geberapparat kann auch eine Kontaktvorrichtung verbunden werden, die bei jeder Bewegung des Geberankers ein Weckersignal einschaltet. Es ertönt dann bei jeder Befehlsabgabe ein Achtungssignal. Die Úbereinstimmung der Anzahl der Glockenschläge mit der Nummer des Befehlsfeldes, wie dies beim Sechsspulenmotor möglich ist, kann allerdings hierbei nicht erreicht werden. 


\section{Vorzeichnis der Tafeln.}

Tafel 1. Graphische Darstollung der Vorgänge beim Parallelschalton Seite

Tafel 2. Ausführungsmöglichkeiten der Dunkelschaltung . . . . 8

Tafel 3. Ausführungsmöglichkeiten dor Hellschaltung . . . . . 10

Tafel 4. Schaltungen mit Umkehrtransformator . . . . . . . 13

Tafel 5. Besondere Drohstrom-Schaltungen .......... 15

Tafel 6. Elektrischo Einstellvorrichtung für den Regulator der Antriebs-Maschine. . . . . . . . . . 20

Tafel 7. MeBwerke der Zungenfrequenzmesser ........ 22

Tafel 8. MeBworko der Spannungsmesser . . . . . . . . . . 24

Tafel 9. Darstellung der Spannungs- und Lichtrerhāltnisse im Dreilampen-Apparat . . . . . . . . . . . . . . 28

Tafol 10. Bauart und Wirkungsweise des Sechslampen-Apparates - 31

Tafel 11. Nullspannungsmesser mit Vorschaltlampe ...... 34

Tafol 12. MeBwerk und Schaltung des Summenspannungsmessers . 36

Tafol 13. Weston-Synchronoskop mit schwingendem Zoiger . . . . 39

Tafel 14. Graphische Darstellung der Arbeitsweiso des Weston-Synchronoskops . . . . . . . . . . . . . . . 41 4

Tafel 15. Siemens-Synchronoskop mit umlaufendem Zeiger. . . . . 44

Tafol 16. Synchronoskop von Hartmann und Braun . . . . . . 46

Tafel 17. Instrumentsätzo für Hellschaltung . . . . . . . . . 48

Tafel 18. Instrumentsätze für gemischte Schaltung . . . . . . . . 50

Tafel 19. Steckvorrichtungen und Stecker . . . . . . . . 54

Tafel 20. Hochspannungsschaltungen mit Meßkondensatoren . . . 104

Tafel 21. Schaltung der Haupttransformatoren nach Schaltart A . . 108

Tafel 22. Schaltung der Haupttransformatoron nach Schaltart 13 . 109

Tafol 23. Schaltung der Haupttransformatoron nach Schaltart C . . 110

Tafel 24. Schaltung der Haupttransformatoren nach Schaltart D . 111

Tafel 25. Darstellung der olektrischen und magnetischen Verhältnisse im Schaltmotor ............. . 117

Tafel 26. Einrichtung zum selbsttätigen Parallelschalten nach Dr. Michalko . . . . . . . . . . . . . 119

Tafel 27. Einrichtung zum selbsttātigen Parallelschalten mit selbsttätiger Regelung dor Antriebsmaschine ........ . 123

Tafel 28. Zeigerbefehlsapparat mit Sechsspulenmotor für Gleichstrom 130

Tafel 29. Zeigerbefehlsapparat mit Dreispulenanker für Gleichstrom . 133

Tafel 30. Zeigerbefehlsapparat nach dem Wechselstromsystem . . 136 


\section{Verzeichnis der Schaltbilder vollständiger Parallelschalteinrichtungen.}

Phasenvergleichung zwisehen Generator und Sammelschienen.

Schaltungen mit Nullspannungsmesser.

Schaltbild 1. Direkte Schaltung . . . . . . . . . 64

Schaltbild 2. Halbindirekto Schaltung ........... 65

Schaltbild 3. Indirekto Schaltung für Spannungen bis 250 Volt . . 66

Schaltbild 4. Indirekto Schaltung für höhero Spannungen . . . 67

Schaltbild 5. Indirekto Schaltung für Doppelsammelschienen . . . 68

Schaltungen mit Summenspannungsmesser.

Schaltbild 6. Direkto Schaltung . . . . . . . . . . . . . . . 69

Schaltbild 7. Halbindirckto Schaltung .......... . 70

Schaltbild 8. Halbindirekte Schaltung mit Instrumentumschalter . 71

Schaltbild 9. Indirekte Schaltung für Spannungen bis 250 Volt . . 72

Schaltbild 10. Indirekte Schaltung für höhere Spannungen . . . . 73

Schaltbild 11. Indirekto Schaltung für Doppclsammelschienen . . . 74

Schaltungen mit Lamponapparat.

Schaltbild 12. Direkte Sclialtung mit Lampenapparat in Dunkelschaltung . . . . . . . . . . . 78

Schaltbild 13. Indirekte Schaltung mit Lampenapparat in Dunkelschaltung . . . . . . . . . . 79

Schaltbild 14. Indirekte Schaltung mit Lampenapparat in Hellschaltung ............. 80

Schaltungen mit Synchronoskop.

Schaltbild 15. Direkte Schaltung . . . . . . . . . . 81

Schaltbild 16. Indirckto Schaltung . . . . . . . . . . 82

Schaltbild 17. Indirekte Schaltung für Doppelsammelschienen . . . 83

Phasenvergleichung zwischen Generator und Generator.

Schaltungen mit Nullspannungsmesser.

Schaltbild 18. Dunkelschaltung für Einfachsammelschienen . . . 88

Schaltbild 19. Dunkelschaltung für Dopnelsammelschienen . . . . 89

Schaltbild 20. Gemischte Schaltung . . . . . . . . . . 90 
Schaltungen mit Summenspannungsmesser.

Schaltbild 21. Unkchrschaltung . . . . . . . . . . . . 91

Schaltbild 22. Umkehrschaltung mit Instrumentumschaltor . . . . 92

Schaltungen mit Synchronoskop.

Schaltbild 23. Schaltung für Einfachsammelschienen . . . . . . 93

Schaltbild 24. Schaltung für Doppelsammelschienen . . . . . . . 94

Schaltbild 25. Gemischte Schaltung . . . . . . . . . . . . . 95

Phasentergleichung an den Sehalterkontakten.

Schaltungen mit Nullspannungsmesser.

Schaltbild 26. Dunkelschaltung für Doppelsammelschienon . . . . 98

Schaltbild 27. Gemischte Schaltung . . . . . . . . 99

Schaltung mit Summenspannungsmesser.

Schaltbild 28. Umkehrschaltung . . . . . . . . . 100

Schaltungen mit Synchronoskop.

Schaltbild 29. Dunkelschaltung . . . . . . . . . 101

Schaltbild 30. Gemischte Schaltung . . . . . . . . 102 


\title{
Meßgeräte und Schaltungen für Wechselstrom- Leistungsmessungen
}

\author{
Von \\ Werner Skirl \\ Oberingenieur
}

Zweite, umgearbeitete und erweiterte Auflage

Mit 41 Tafeln, 31 ganzseitigen Schaltbildern und zahlreichen Textbildern 1923. Gebunden G\%. 6

Elektrotechnische Me@kunde. Von Dr.-Ing. P. B. Arthur Linker. Dritte, rülig ungearbeitete und erweiterte Auflage. Mit 408 Textfiguren. Unverlinderter Neudruck. 1923. Gebunden GZ. etwa 11

Elektrotechnisehe Meßinstrumente. Ein Leitfaden. Von Kourad Gruhn, Oberingenieur nnd Gewerbestudienrat. $Z$ weite, vermehrte und verbesserte Auflage. Mit 321 T'extabbildungen. 1923.

Gebunden GZ. 5.8

Messungen an elektrischen Maschinen. Apparate, Instrumente, Methoden, Schaltungen. Von Rud. Krause r. Funfte, vermehrte und verbesserte Auflage ron Georg Jahn, Diplomingenieur. Mit etwa 250 Textabbildungen.

In Vorbereitung

Der Wechselstromkompensator. Von Dr.-Ing. W. v. Krukowski. Mit 20 Abbildungen im Text und auf einem Textblatt. (Sonderabdruck aus der Abhandlung "Vorgänge in der Scheibe eines Induktionszihlers und der Wechselstromkompensator als Hilfsmittel zu deren Erforschung".) 1920.

GZ. 3.8

Comparison of Principal Points of Standards for Electrical Machinory. (Rotating Machines and Transformers). By Dipl.-Ing. Friedrich Nettel, Charlottenburg. 1923 . GZ. 2.25; gebunden GZ. 3

Die Grundzahlen (GZ.) entsprechen den ungefahren Forkricgspreisen und ergeben mü dem jeweiligen Entwertungsfaktor ( Umrechnungsschlüssel) vervielfacht den Ferkaufspreis. Uber den zur Zeit geltenden Umrechnungsechlüssel geben alle Buchhandiungen sovie der Verlay bereituilligat Auskunft. 
Hilfsbuch für die Elektrotechnik. Unter Mitwirkung zahlreicher Fachgenossen bearbeitet und herausgegeben ron Professor Dr. Karl Strecker, Geh. Oberpostrat, Berlin. Z e h $n$ te, umgearbeitete Auflage. In drei Teilen.

In Vorbereitung

IKurzes Lehrbuch der Elektrotechnik. Von Dr. Adolf Thomailen, a. 0. Professor an der Technischen Hochschule Karlsruhe. Neunte, verbesserte Auflage. Mit 555 Textbildern. 1922. Gebunden GZ. 9

Die wissensehaftlichen Grundlagen der Elektrotechnik. Von Professor Dr. Gustar Benischke. Sechste, vermehrte Auflage. Mit 633 Albbildungen im Text. $1922 . \quad$ Gebunden GZ. 15

Küurzer Leitfaden der Elektrotechnik fur Unterricht und Praxis in allgemeinverstundlicher Darstellung. Von Ingenieur Rud. Krause. $V$ ierte, rerbesserto Auflage, herausgegeben von Prof. H. Vieweger. Mit 375 Textfiguren. 1920.

Gebunden GZ. 6

Dio Elcktrotechnik und dio elcktromotorischen Antriebe. Ein elementares Lehrbuch fur technische Lehranstalten und zum Selbstunterricht. Von Dipl.-Ing. Wilhelm Lchmann. Nit 520 Textabbildungen und 116 Beispielen. $1922 . \quad$ Gebunden GZ. 9

Elektrische Starkstromanlagen. Maschinen, Apparate, Schaltungen, Betrieb. Kurzgefa Btes Hilfsbuch fur Ingenieure und Techniker sowio zum Gebrauch an technischen Lehranstalten. Von Studienrat Dipl.Ing. Emil Kosack, Maydeburg. Sechste, durchgeselene und erginzte Auflage. Mit 296 Textfiguren. 1923. GZ. 5 ; gebunden GZ. 5.8

Schaltungen von Gleich- und Wechselstromanlagen. Dynanomaschinen, Motoren und Transformatoren, Lichtanlagen, Kraftwerke und Umformerstationen. Ein Lehr- und Hilfsbuch. Von Studienrat Dipl--Ing. Emil Kosnek, Magdeburg. Mit 226 Textabbildungen. 1922.

GZ. 4 ; gebunden GZ. 6

Elektrische Schaltvorgänge und verwandte Störungserscheinungen in Starkstromanlagen. Von Professor Dr.-Ing. und Dr.-Ing. e. h. Reinhold Rüdenberg, Priratdozent, Berlin. Mit $477 \mathrm{Ab}$ bildunger im Text und 1 Tafel. $1923 . \quad$ Gebunden GZ. 16

Grundzüge der Starkstromtechnik. Fur Unterricht und Praxis. Von Dr.-Ing. K. Hoerner. Mit 319 Textabbildungen und zahlreichen Beispielen. 1923. GZ. 4 ; gebunden GZ. 5

Die Grundzahlen (GT.) entsprechen den unge/ühren Vorkriegspreisen und ergeben mit dem jeweiligen Entuertunosfaktor (Umrechnungsschlilssel) verviel/acht den Verkau/spreis. Uber den zur Zeit geltenden Ëmrechnungssclüssel geben alle Buchhandlungen souie der Verlog bereitoilligat Auskuntt. 
Arnold-la Cour, Die Wechselstromtechnik. Herausgegeben von Professor Dr.-Ing. E. Arnold, Karlsruhe. In 5 Bünden. Unverdinderter Neudruck.

Erscheint Anfang Sommer 1923

Theorie der Wechselströme. Von Dr.-Ing. Alfred Frnenckel. $Z w$ oite, erweiterto und verbesserte Auflage. Mit 237 Textfiguren. 1921.

Gebunden GZ. 11

Aufgaben und Lösungen aus der GIeich- und Wechselstromtechnik. Ein Übungsbuch fur den Unterricht an techuischen Hochund Fachschulen sowie zum Selbststudium. Von Professor H. Vieweger. A chto Auflage. Mit 210 Textfiguren und 2 Tafeln. 1923. GZ. 4; gebunden GZ. 5

Ankerwicklungen für Gleich- und Wechselstrommaschinen. Ein Lehrbuch. Yon Professor Rudolf Richter, Karlsruhe. Mit 377 T'extabbildungen. Berichtigter Neudruck. 1922.

Gebunden GZ. 11

Die symbolische Methode zur Lösung von Wechselstromaufgaben. Einfuhrung in den praktischen Gebrauch. Vun Hugo Ring, Ingenieur, Hamburg. Mit 33 Textfiguren. 1921. GZ. 2.3

Dio Berechnung von Gleich- und Wechselstromsystemen. Neue Gesetze uber ihre Leistungsaufnahme. Von Dr.-Ing. Fr. Natalis. Mit 19 Textfiguren. 1920.

GZ, 1

Die Hochspannungs-Gleichstrommaschine. Eine grundlegende Theorie. Von Dr. A. Bolliger, Elektro-Ingenieur in Zürich. Mit 53 Textfiguren. 1921.

GZ. 2

Arnold-Ia Cour, Die Gleichstrommaschine. Ihre Theorie, Untersuchung, Konstruktion, Berechnung und Arbeitsweise.

Erster Band: Theoric und Untersuehung. Von J. L. In Cour. D ritte, vollständig umgearbeitete Auflage. Mit 570 Textfiguren. Unverönderter Neudruck. 1923. Gebunden GZ. 18 $Z$ weiter $B$ and: Konstrultion, Berechnung und Arbeitsweise. In Vorbereitung

Der Drehstrommotor. Ein Handbuch fur Studium und Praxis. Von Professor Julius Heubach, Direktor der Elektromotorenwerke Heidenau, G. m. b. H. Z w o ite, verbesserte Auflage. Mit $222 \mathrm{Ab}$ bildungen. 1923. Gebunden GZ. 14.5

Elektromotoren. Ein Leitfaden zum Gebrauch fur Studierende, Betriebsleiter und Elektromonteure. Von Dr.-Ing. Johann Grabscheid. Mit 72 Textabbildungen. 1921.

GZ. 2.8

Die Grundzahlen (GZ.) entsprechen den ungefahren Forkriegspreisen und eroeben mü dem jeweiligen Entocrungsfaktor (Omrechnungsschlissel) veroielfacht den Verhaufspreis. Uber den zur Zeit geltenden Umrechnungsechlüsel oeben alle Buchhandlungen sowie der Ferlag bereitcilligst Aluskunt. 


\section{Die Elektromotoren in ihrer Wirkungsweise und Anwendung.}

Ein Hilfsbuch fur die Auswahl und Durchbildung elektromotorischer Antriebe. Von Karl Neller, Oberingenieur. Z w e ite, vermehrte und verbesserte Auflage. Mit 297 Textabbildungen. Erscheint im Juli 1923

Die Transformatoren. Von Professor Dr. techn. Milan Vidmar. $\mathrm{Z}$ we it e Auflage. Nit etwa 297 Textabbildungen. In Vorbereitung

Die asynchronen Wechselfeldmotoren. Kommutator- und Induklionsmotoren. Von Professor Dr. Gustar Benischke. Mit $89 \mathrm{Ab}$ bildungen im Text. 1920.

G $/ .3 .5$

Elektrische Durchbruchfeldstärke von Gasen. Theoretische Grundlagen und Anwendung. Von W. O. Seliumann, a. o. Professor der technischen Physik an der Unirersitat Jena. Mit 80 Textabbildungen. 1923.

GZ. 6; gebunden G7. 7.25

Hochfrequenzmeßtechnik. Ihre wissenschaftlichen und praktischen Grundlagen. Von Dr.-Ing. A ugust Hund, beratender Ingenieur. Mit 150 Textabbildungen. 1922.

Gebunden GZ. 8.t

Die Nebenstellentechnik. Von Hans B. Willers, Oberingenieur und Prokurist der Aktiengesellschaft Mix \& Genest, Berlin-Schüneberg. Mit 137 Textabbildungen. 1920.

Gebunden GZ. 6

Anleitungen zum Arbeiten imElek trotechnischen Laboratorium. Von E. Orlich. Erster Teil. Mit 74 Textbildern. 1923.

G\%. 2

Handbuch der drahtlosen Telegraphie und Telephonie. Ein Lehrund Nachschlagebuch der dralttlosen Nachrichtenulermittlung. Von Dr. Fugen Nesper. Z we i Bănde. Z wi eite, neubearbeitete und ergänzte Auflage.

In Vorbereitung

Radiotelegraphisches Praktikum. Von Dr.-Ing. Ir. Rein. Dritte, ungearbeitete und vermelirte $\Lambda$ uflage von Professor Dr. K. Wirtz, Darmstadt. Mit 432 Textabbildungen und 7 Tafeln. Berichtigter Neudruck. 1922.

Gebunden GZ. 16

Die Grundzahten (GZ.) entsprechen den ungeführen Vorkricospreisen und ergeben mit dem jeweiligen Entuertungs/aktor ( Dnrachuunjsschlüssel) vervieljashl den Verkau/spreis. Uber den zur Zeit geltenden Umrechnungsschlüssel geben alle Buchhandlungen sovie der Ferlag bereituilligst Auskundt. 
BG Politechniki Śląskiej

$\mathrm{nr}$ inw.: 102 - 130362

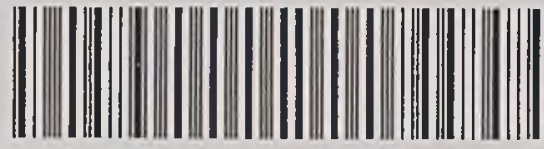

Dyr.1 130362 